\title{
Is de-carbonized development possible? Household emissions and renewable energy in developing countries
}

\author{
Dissertation \\ zur Erlangung des wirtschaftswissenschaftlichen Doktorgrades \\ der Wirtschaftswissenschaftlichen Fakultät der Universität Göttingen
}

\author{
vorgelegt von \\ Moises Neil V. Seriño \\ aus Leyte, Philippines
}

Göttingen, Germany

October 2014 
Erstgutachter: $\quad$ Prof. Stephan Klasen, Ph.D.

Zweitgutachter: $\quad$ Prof. Inmaculada Martínez-Zarzoso, Ph.D.

Drittprüfer: $\quad$ Prof. Dr. Thomas Kneib

Tag der mündlichen Prüfung: 20.10.2014 


\section{Summary}

Today's policy makers are facing the challenge of mitigating climate change without limiting the growth potentials of developing countries. In this vein, this study offers a step towards answering the question is de-carbonized development possible. Particularly, we investigate household emissions and the potential diffusion of renewable energy in developing countries. This study contributes to the literature in four main points. First, it investigates household carbon emissions from a developing country's perspective and analyzes the influence of rising income on emissions while controlling for households sociodemographic characteristics. Second, it explores other relevant factors such as carbon intensity and energy intensity that could influence rising emissions. Third, it examines how unequal the households are in their emissions. Emission inequality has direct implications towards reducing household carbon emissions. Lastly, this study investigates the potential diffusion of various sources of renewable energy in developing countries. Renewable energy appears to be a feasible approach in reducing carbon emissions. It can help fuel growth in developing countries without further aggravating the alarming concentration of green house gas emissions accumulated in the atmosphere.

The first essay aims to answer the question, how carbon intensive is the lifestyle of Philippine households and investigates the possibility of delinking affluence and household emissions. We estimate household carbon emissions embodied in various consumptions of goods and services by combining input-output analysis with household expenditure for 2000 and 2006. Based on the estimation, expenditures related to fuel, light and transportation are the most carbon intensive goods consumed by households while nondurable goods are the least carbon intensive. Key results show that while households' socio-demographic characteristics matter in explaining emissions, we found no concrete evidence on delinking household affluence and emissions. Unless consumption patterns changes, it is likely that Philippines households will lead a carbon intensive lifestyle, as households get richer.

The second essay decomposes the changes in household emissions and investigates other relevant factors such as carbon intensity and energy intensity that could influence household emissions. While the first paper points out the strong correlation between emissions and income, decomposing the change in emission shows that this correlation varies across household distributions. The income effect is more pronounced among poor households while the energy intensity effect is more pronounced among rich households. This suggests that improving energy intensity can be a feasible option in reducing household emissions, in particular, promoting the use of energy efficient household appliances, and use of fuel-efficient cars or access to improved public transportation.

If aiming to reduce household carbon emissions, then it is necessary to examine how unequal the households are in their emission levels. Any climate mitigation policies aimed at reducing emissions has a more pronounced effect in a more equal society than in anequal one. Results show that there is a high and rising emission inequality among households and a 
bigger portion of the emission inequality is explained by energy intensive household consumption such as fuel, light and transportation. This suggests that for targeting purposes policy makers should focus on these energy intensive consumptions if aiming to control household emission inequality.

The first three essays highlight that a large share of the total household carbon emissions is due to energy intensive consumption. This suggests that shifting of energy sources to emission-neutral sources such as renewable energy is crucial in maintaining or improving household lifestyle without contributing to further increases in global emissions. Hence, for the fourth essay we model the potential diffusion of various sources of renewable energy in developing countries and investigate its determinants. We focus on diversification because most renewable energy rely on the weather as its main source and these sources are unpredictable but diversification can allow for a steady and reliable supply of energy. Results show a robust nonlinear effect of income on diversification depicting a U-shape kind of relationship. In addition, without relying on foreign direct investments and development assistance, we find that developing countries with technological advances, skilled human capital, developed financial markets, sound governance and greater renewable energy potential can move to diversification of renewable energy sources. We also document a wider diversification of renewable energy sources since the adoption of Kyoto Protocol in the late 1997.

Based on the evidence presented above the following policy implications can be drawn. On the household side, while it is unlikely that households will lead a low carbon lifestyle as they become more affluent and imposing restrictions on what households can consume is difficult and controversial, taxing carbon intensive goods can be an option if aiming to control household emissions. However by doing this, policy makers should be cautious not to jeopardize the efforts in reducing poverty in the Philippines where a quarter of its population lives below poverty line. Several other options are also possible in curbing household carbon emissions. These include improving production efficiency and changing consumption patterns to less carbon-intensive lifestyles and in particular, improvements in access to efficient public transport, to energy efficient lighting and cooling technologies. In addition, increasing use of renewable energy sources and integrating renewable energy in developing countries' energy mix is an important policy agenda to help decouple economic growth with emissions. 


\section{Zusammenfassung}

Politische Entscheidungsträger stehen heute vor der Herausforderung, dem Klimawandel zu begegnen, ohne das Entwicklungspotential von Entwicklungsländern einzuschränken. In diesem Zusammenhang trägt diese Arbeit zur Beantwortung der Frage bei, ob Entwicklung ohne höheren $\mathrm{CO}_{2}$-Ausstoß möglich ist. Untersucht werden insbesondere die Emissionen von Haushalten und die mögliche Verbreitung erneuerbarer Energien in Entwicklungsländern. Diese Arbeit geht in vier Punkten über die bisherige Literatur hinaus. Erstens untersucht sie aus der Perspektive eines Entwicklungslandes die $\mathrm{CO}_{2}$-Emissionen von Haushalten und analysiert den Einfluss steigender Einkommen auf Emissionen, unter Berücksichtigung sozio-demografischer Eigenschaften der Haushalte. Zweitens betrachtet sie andere relevante Faktoren wie $\mathrm{CO}_{2}$-und Energieintensität, die steigende Emissionen beeinflussen könnten. Drittens untersucht sie, wie ungleich die Emissionen der Haushalte verteilt sind. Aus der Ungleichheit von Emissionen ergeben sich direkte Konsequenzen für die Reduzierung der $\mathrm{CO}_{2}$-Emissionen von Haushalten. Zuletzt wird die Möglichkeit der Verbreitung verschiedener erneuerbarer Energien in Entwicklungsländern erforscht. Erneuerbare Energien scheinen eine Handlungsoption zur Reduzierung von $\mathrm{CO}_{2}$-Emissionen darzustellen. Sie können helfen, Wachstum in Entwicklungsländern zu fördern, ohne die bereits besorgniserregend hohe Konzentration von klimaschädlichen Gasen in der Atmosphäre weiter zu verschlimmern.

Der erste Teil der Dissertation untersucht, wie $\mathrm{CO}_{2}$-intensiv der Lebensstil philippinischer Haushalte ist und analysiert Möglichkeiten, Emissionen und Wohlstand von Haushalten zu entkoppeln. Wir schätzen die $\mathrm{CO}_{2}$-Emissionen der Haushalte, die durch den Konsum verschiedener Güter und Dienstleistungen verursacht werden, indem wir eine InputOutput-Analyse mit den Ausgaben der Haushalte in den Jahren 2000 und 2006 kombinieren. Auf Basis der Schätzung sind die Ausgaben der Haushalte, die im Zusammenhang mit Kraftstoffen, Licht und Transport stehen, die $\mathrm{CO}_{2}$-intensivsten, während diejenigen für kurzlebige Güter am wenigsten $\mathrm{CO}_{2}$-intensiv sind. Die zentralen Ergebnisse sind, dass während sozio-demografische Eigenschaften der Haushalte wichtig für die Erklärung der Höhe von Emissionen sind, keine konkreten Anzeichen für eine Entkopplung von $\mathrm{CO}_{2^{-}}$ Emissionen und Wohlstand gefunden wurden. Wenn sich das Konsumverhalten nicht ändert, werden philippinische Haushalte bei steigendem Wohlstand wahrscheinlich einen Lebensstil führen, der mit höherem $\mathrm{CO}_{2}$-Ausstoß einhergeht.

Der zweite Teil schlüsselt die Veränderung der Emissionen durch Haushalte auf und untersucht andere relevante Einflussfaktoren wie $\mathrm{CO}_{2}$-Intensität und Energieintensität. Während der erste Teil die starke Korrelation zwischen Emissionen und Einkommen herausarbeitet, wird durch die Aufschlüsselung deutlich, dass diese Korrelation nicht gleichverteilt zwischen allen Haushalten ist. Der Einkommenseffekt ist in ärmeren Haushalten ausgeprägter, während der Effekt der Energieintensität in reicheren Haushalten überwiegt. Dies deutet darauf hin, dass die Energieintensität ein Ansatzpunkt dafür sein kann, Emissionen von Haushalten zu reduzieren. Insbesondere kann der Einsatz energieeffizienter Haushaltsgeräte gefördert werden, ebenso wie kraftstoffsparende Fahrzeuge oder der Zugang zu öffentlichen Transportmitteln. 
Um die CO2-Emissionen von Haushalten zu reduzieren, muss untersucht werden, wie sich die Emissionen auf die Haushalte verteilen. Jede politische Maßnahme zur Linderung des Klimawandels, die die Reduktion von Emissionen beinhaltet, hat einen direkteren Einfluss in gleicheren Gesellschaften, als in Ungleicheren. Die Ergebnisse zeigen, dass sich die Emissionen sehr ungleich zwischen den Haushalten verteilt sind, dass diese Ungleichheit zunimmt und sich ein großer Anteil der Emissionsungleichheit durch energieintensiven Konsum von Kraftstoffen, Licht und Transport erklärt. Dies legt nahe, dass politische Entscheidungsträger sich auf energieintensiven Konsum fokussieren sollten, um die Ungleichheit von Emissionen zu reduzieren.

Die ersten drei Teile der Arbeit stellen heraus, dass ein großer Anteil der CO2Emissionen der Haushalte durch energieintensiven Konsum verursacht wird. Dies deutet auf die Notwendigkeit hin, auf emissionsneutrale Energiequellen wie erneuerbare Energien umzusteigen um den Lebensstil der Haushalte zu erhalten oder zu verbessern ohne die globalen Emissionen weiter ansteigen zu lassen. Daher wird im vierten Teil die potentielle Ausbreitung verschiedener erneuerbarer Energiequellen in Entwicklungsländern modelliert und ihre Bestimmungsfaktoren untersucht. Wir konzentrieren uns auf die Diversifikation, da die meisten erneuerbaren Energien wetterabhängig und dadurch nicht planbar sind, eine Diversifikation aber eine stabile und verlässliche Energieversorgung ermöglicht. Die Ergebnisse zeigen einen robusten nichtlinearen Effekt von Einkommen auf Diversifikation, der sich als grafisch als U-förmige Beziehung zeigt, so dass wir eine weitere Diversifikation erneuerbarer Energiequellen bei steigenden Einkommen in Entwicklungsländern erwarten. Darüber hinaus können technologisch fortschrittlichere Entwicklungsländer, Entwicklungsländer mit ausgebildeten Fachkräften, entwickelten Finanzmärkten, guter Regierungsführung und hohem Rohstoffvorkommen den Einsatz erneuerbarer Energien diversifizieren ohne auf ausländische Direktinvestitionen und Entwicklungshilfe angewiesen zu sein. Wir dokumentieren ferner, dass sich die Diversifizierung erneuerbarer Energiequellen seit Einführung des Kyoto-Protokolls Ende 1997 weiter verbreitet hat.

Auf Grundlage der obigen Erkenntnisse können die folgenden Politikempfehlungen abgeleitet werden. Auf Seiten der Haushalte: Es ist unwahrscheinlich, dass Haushalte bei steigendem Wohlstand einen Lebensstil führen werden, der mit geringem CO2-Ausstoß einhergeht. Den Konsum der Haushalte zu beschränken ist jedoch schwierig und umstritten. Güter, durch die ein hoher CO2-Ausstoß verursacht wird, können besteuert werden um die Emissionen von Haushalten einzuschränken. Allerdings sollten politische Entscheidungsträger dabei Vorsicht walten lassen, um nicht die Bemühungen der Armutsbekämpfung zu gefährden, da auf den Philippinen ein Viertel der Bevölkerung als arm anzusehen ist. Es gibt verschiedene andere Optionen, die CO2-Emissionen der Haushalte zu drosseln. Dazu zählt die Verbesserung der Produktionseffizienz und die Veränderung des Konsumverhaltens hin $\mathrm{zu}$ einem weniger CO2-intensiven Lebensstil. Wichtig sind dabei insbesondere Verbesserungen beim Zugang zu öffentlichem Verkehrsmitteln, sowie zu energieeffizienteren Beleuchtungs- und Kühltechnologien. Ferner ist es ein wichtiger Politikansatz, den Einsatz erneuerbarer Energiequellen auszuweiten und erneuerbare Energie in den Energiemix von Entwicklungsländern einzugliedern, um wirtschaftliches Wachstum von Emissionen zu entkoppeln. 


\section{Acknowledgements}

I would like to express my deepest appreciation to my $\mathrm{PhD}$ committee chair, Prof. Stephan Klasen, for agreeing to supervise my research work paving the way for this wonderful opportunity to study in Göttingen University and for all the advices and guidance throughout the completion of my dissertation. I would also like to extend my sincere gratitude to my thesis committee members, Prof. Inmaculada Martinez-Zarzoso and Prof. Thomas Kneib, for sharing their expertise, for their helpful comments and suggestions. Thank you very much.

I would like to acknowledge the financial support from the EXPERTS I - Exchange by Promoting Quality Education, Research \& Training in South and South-East Asia. Without the scholarship, it would have been impossible for me to pursue my PhD study in Göttingen, Germany. In addition, I would like to thank EntDekEn Project and the Chair of Development Economics for the additional funding in carrying out my research.

I am thankful to Mr. Ballester for his support during data collection and to Jennifer for proofreading my work. I also have to thank my colleagues Bumi, Iqbal and Jan for making $\mathrm{PhD}$ life more interesting. To the Filipino community in Göttingen for making me feel at home, to my friends and colleagues in the Chair of Development Economics, and to those who in one way or another have helped me in challenging times and supported me throughout the PhD process, thank you very much. Lastly, I want to thank my parents for all the prayers and encouragements and to Eunice for the overwhelming support despite the distance.

Vielen Dank!

Above all, I give thanks to God. 


\section{Contents}

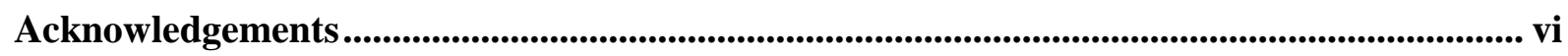

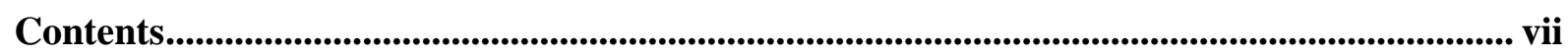

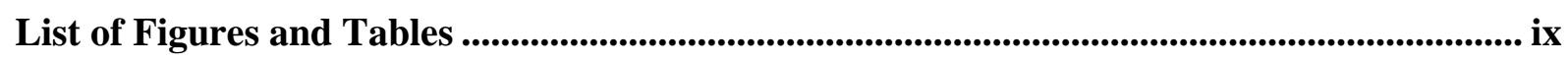

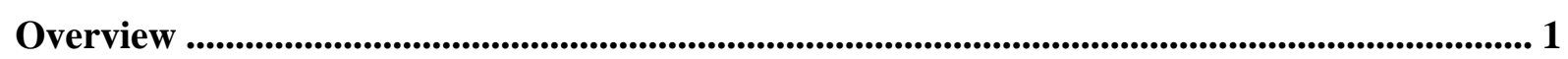

Essay 1: Do Philippine households lead a carbon intensive lifestyle? ....................................... 5

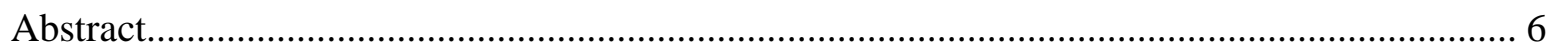

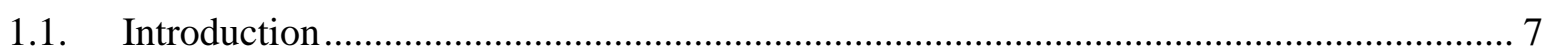

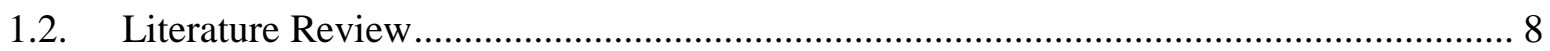

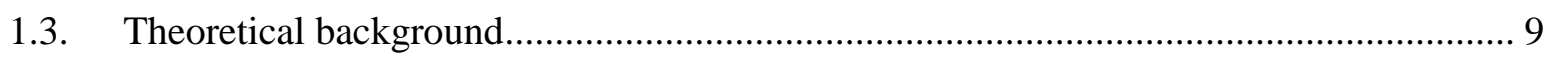

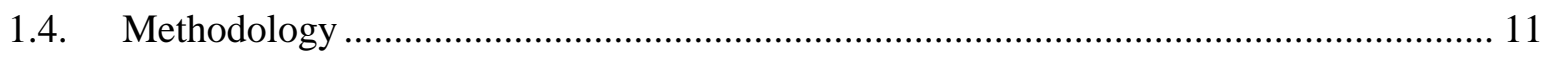

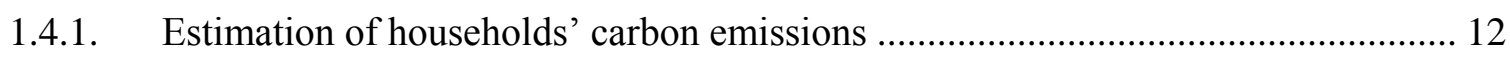

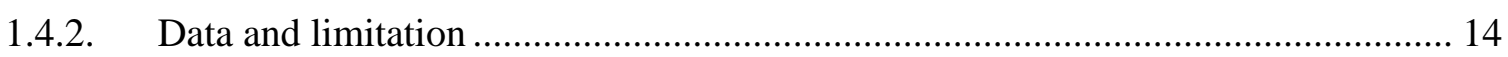

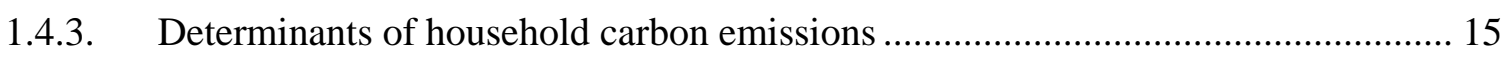

1.4.4. Asset index construction ............................................................................. 17

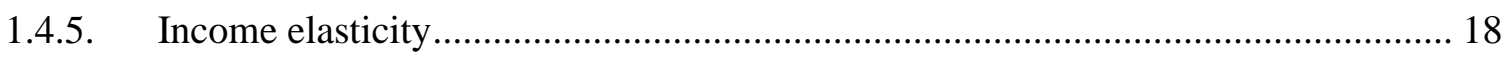

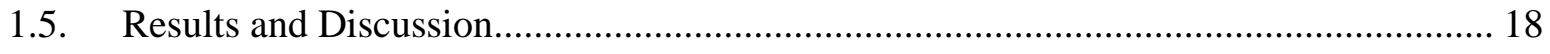

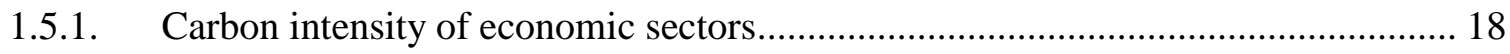

1.5.2. Characteristics of households' carbon emissions .................................................. 19

1.5.3. Household carbon emissions and income............................................................. 22

1.5.4. Carbon emissions and other household characteristics ........................................ 27

1.5.5. Household carbon emissions and asset index......................................................... 29

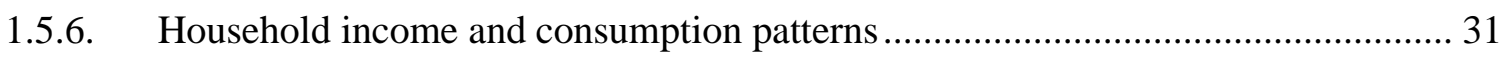

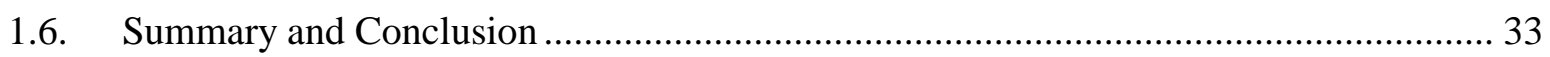

Essay 2: Decomposing drivers of rising household carbon emissions in the Philippines ...... 35

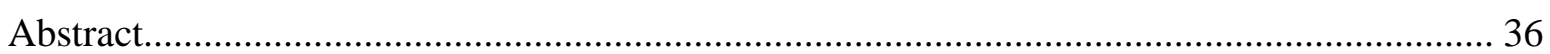

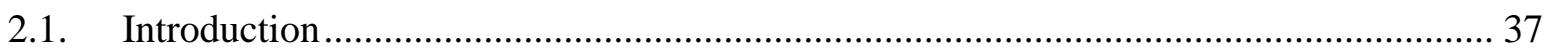

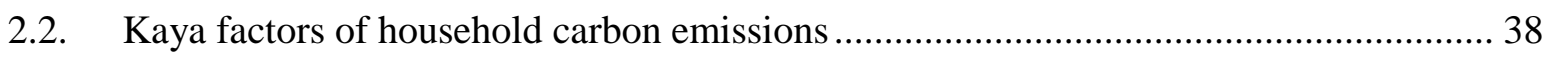

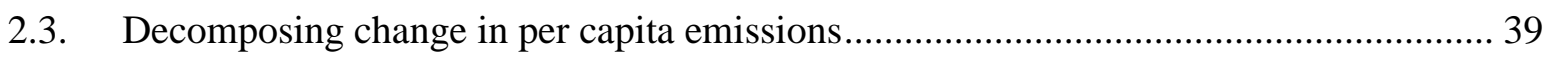

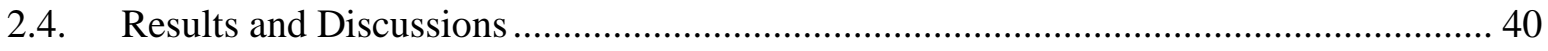

2.4.1. Household characteristics and Kaya factors........................................................ 40

2.4.2. Decomposition of household emissions by Kaya factors ....................................... 41

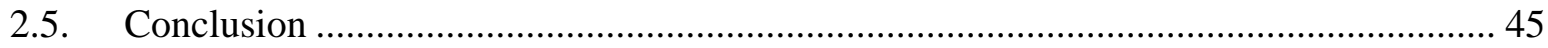




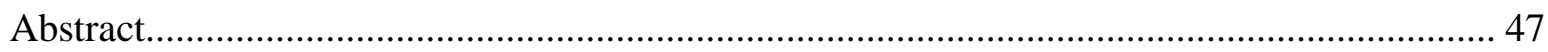

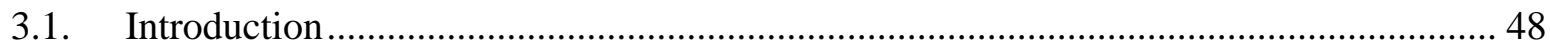

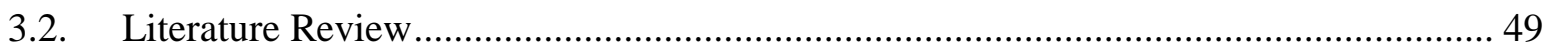

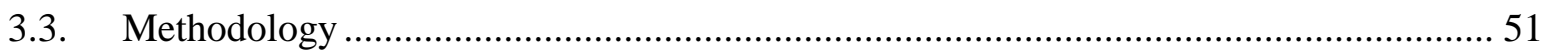

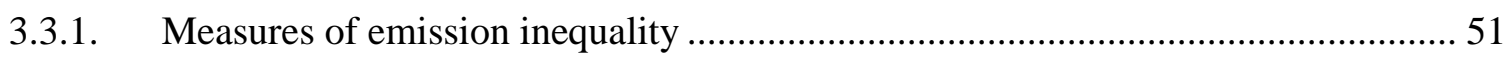

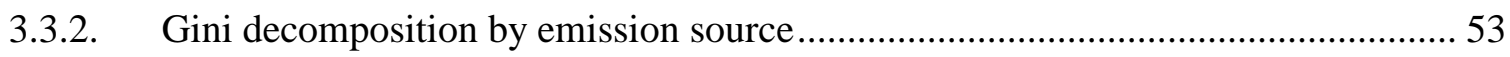

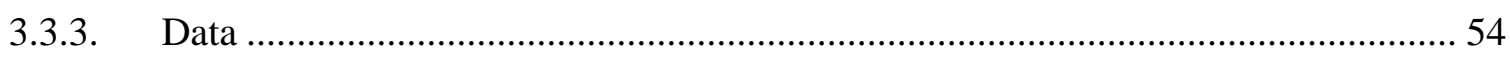

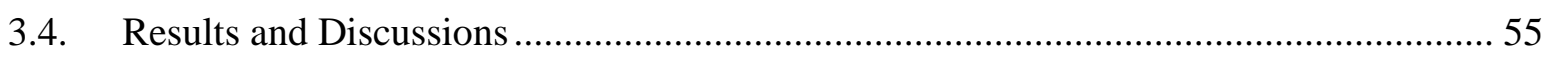

3.4.1. Emissions and income gap between rich and poor households............................. 55

3.4.2. Inequality in households' carbon footprint and its relation to income distribution. 55

3.4.3. Simple inequality in household carbon emissions.............................................. 58

3.4.4. Household characteristics and decomposition of carbon footprint inequality ........ 59

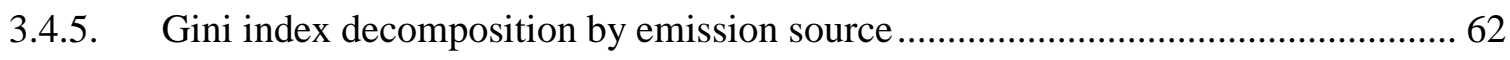

3.4.6. Inequality in major consumption categories across income quintiles ..................... 64

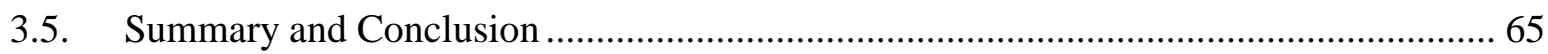

Essay 4: What motivates developing countries to diversify sources of renewable energy? .. 67

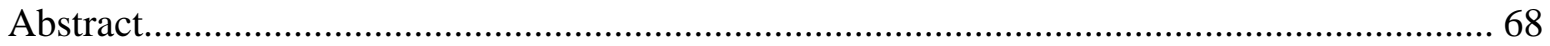

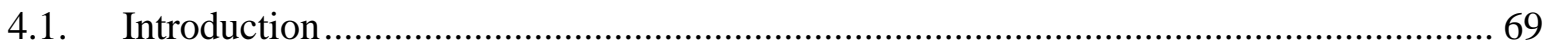

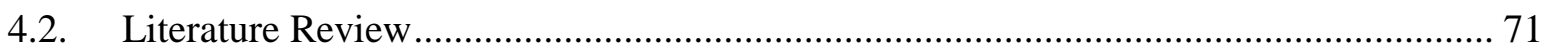

4.3. Theoretical background and diversification of renewable energy mix ......................... 73

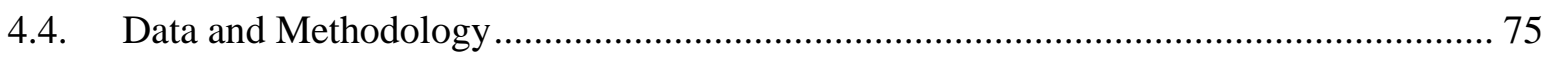

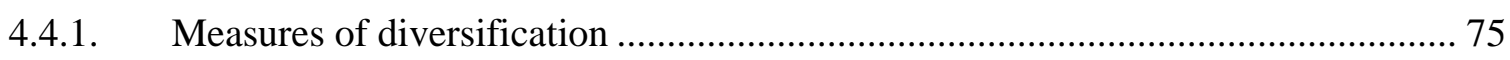

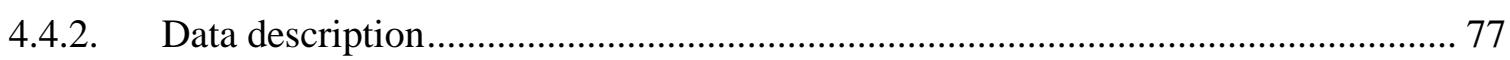

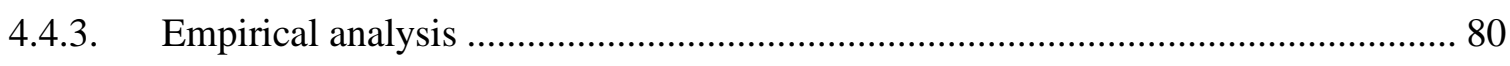

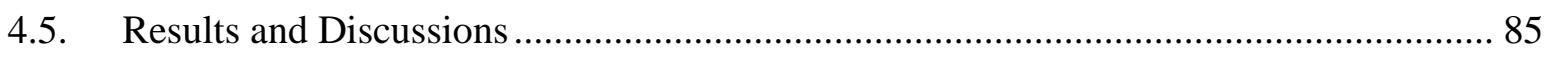

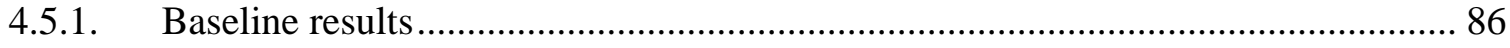

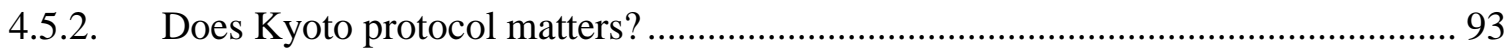

4.5.3. Extended results and robustness check............................................................... 95

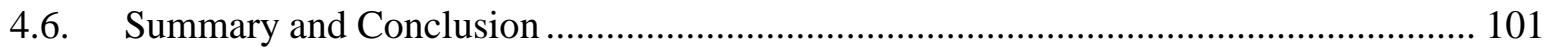

References ........................................................................................................................................................................ 103

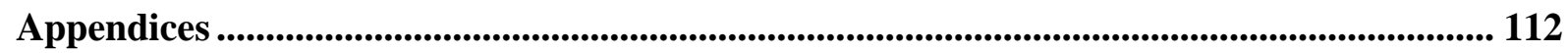




\section{List of Figures and Tables}

\section{Figures}

Figure 1.1. Environmental Kuznets curve for household carbon emission and income.

Figure 1.2. Process flow using the expenditure approach in estimating household carbon emissions. 11

Figure 1.3. Process flow estimation and matching of IO sectors with household consumption. .......... 13

Figure 1.4. Mean per capita $\mathrm{CO}_{2}$ emissions by expenditure categories. ............................................ 20

Figure 1.5. Mean per capita $\mathrm{CO}_{2}$ emissions by income group. ........................................................ 21

Figure 1.6. Mean per capita $\mathrm{CO}_{2}$ emissions by urban-rural location................................................ 22

Figure 2.1. Decomposition of household emissions by Kaya factors across quintiles......................... 43

Figure 2.2. Decomposing change in household emissions across income quintiles by Kaya factors... 44

Figure 3.1. Lorenz curve of income and concentration curve of household carbon emissions............. 58

Figure 3.2. Comparison of inequality in major consumption categories across quintiles.................... 65

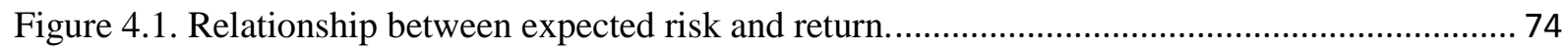

Figure 4.2. Distribution of nonhydro sources of renewable energy ................................................ 82

Figure 4.3. Goodness of fit of Poisson and negative binomial distribution. ....................................... 82

Figure 4.4. Number of countries adopting nonhydro sources of renewable energy.............................. 85

Figure 4.5. Number of countries diversifying sources of renewable energy ...................................... 86

Figure 4.6. Nonhydro renewable energy per capita production (measured in $\mathrm{kWh}$ ). .......................... 95 


\section{Tables}

Table 1.1. Top 20 highest and lowest emitting $\mathrm{CO}_{2}$ sectors........................................................ 19

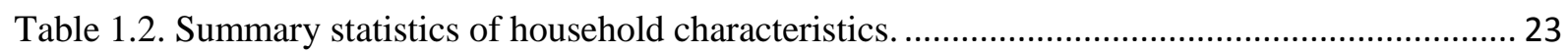

Table 1.3. Factors affecting household $\mathrm{CO}_{2}$ emissions with $\log$ of $\mathrm{CO}_{2}$ as a dependent variable. ....... 26

Table 1.4. Determinants of household emissions using asset index as proxy for income..................... 30

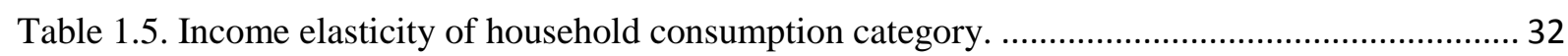

Table 2.1. Kaya factors and household characteristics.

Table 3 1. Comparison of the per capita emissions and income gap across quintiles........................... 55

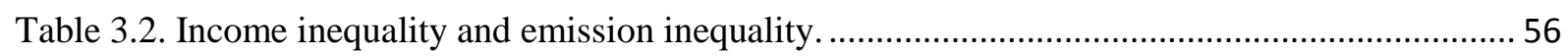

Table 3.3. Concentration indexes and Kakwani index by income quintile. ......................................... 57

Table 3.4. Household inequality index by emissions, income and expenditure.................................... 59

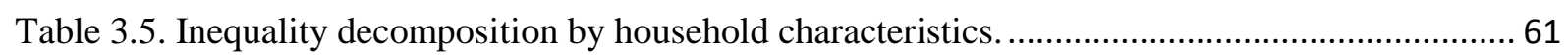

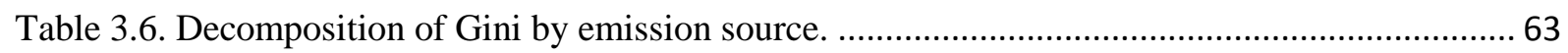

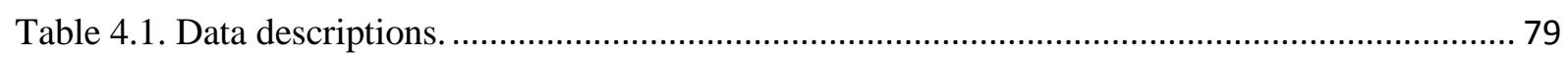

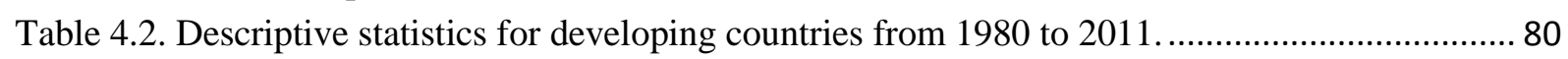

Table 4.3.Baseline analysis using a negative binomial regression..................................................... 90

Table 4.4. Average marginal effects from the two-parts model estimation (TPM). ............................. 92

Table 4.5. Effect of Kyoto Protocol on the diversification of nonhydro sources.................................. 94

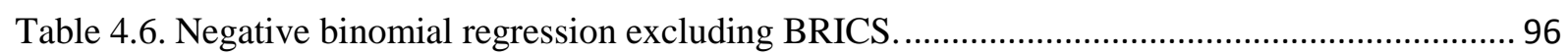

Table 4.7. Robustness check using PPML with diversity index as a dependent variable.................... 98

Table 4.8. Additional robustness check using the Poisson pseudo-maximum likelihood estimation . 100

Appendix Table 1. Matching of Philippine Input-Output sectors with GTAP sectors........................ 112

Appendix Table 2. $\mathrm{CO}_{2}$ emission intensities of different economic sectors. ...................................... 117

Appendix Table 3. Matching of household consumption items with IO sectors................................ 120

Appendix Table 4. Summary statistics of household asset. ............................................................... 126

Appendix Table 5. Household share, mean carbon emission and emission share ............................. 127

Appendix Table 6. Incidence rate ratio for negative binomial regression for baseline analysis......... 128

Appendix Table 7. Incidence rate ratio capturing the effect of Kyoto protocol on diversification ..... 129

Appendix Table 8. Estimation using Two-Parts Model with diversity index as dependent variable.. 130

Appendix Table 9. Estimation using Two-Parts Model controlling for Kyoto Protocol...................... 131

Appendix Table 10. Effect of Kyoto Protocol using time dummies. ................................................. 132 


\section{Is de-carbonized development possible? Household emissions and renewable energy in developing countries}

\section{Overview}

In its latest assessment report, the Intergovernmental Panel for Climate Change (IPCC) said that it is extremely likely that human influence has been the dominant cause of the observed warming since the mid-20 ${ }^{\text {th }}$ century (IPCC 2013a). This global warming phenomenon has been largely attributed to the rising anthropogenic green house gas (GHG) emissions particularly carbon dioxide $\left(\mathrm{CO}_{2}\right)$ emissions resulting from economic activities such as the burning of fossil fuels, industrial processes and other activities. Thomas Stocker, Co-Chair of Working Group 1 of the IPCC said that, "continued emissions of green house gases will cause further warming and changes in all components of the climate system. Limiting climate change will require substantial and sustained reductions of greenhouse gas emissions" (IPCC 2013a).

Historically, developed countries are responsible for the majority of the accumulated green house gas emissions in the atmosphere. However in the last decade, a greater share of the increase in global $\mathrm{CO}_{2}$ emissions has come from developing countries, notably from China and other large developing countries. From 2005 onwards and for the first time in history, the aggregate $\mathrm{CO}_{2}$ emissions coming from developing countries surpassed that of the developed countries (International Energy Agency [IEA] 2013). If other developing countries follow this carbon intensive development pathway, like China did, it will further aggravate the alarming level of $\mathrm{CO}_{2}$ emissions in the atmosphere and jeopardize the efforts in stabilizing the climate system. Developing countries are more likely to be affected by the perpetual adverse effects of this climate change. According to the climate risk index (CRI) developed by Germanwatch, less developed countries are more frequently hit by extreme weather events and are generally more affected than developed countries (Germanwatch 2014).

One of the biggest challenges policy makers face today is stabilizing the climate system without limiting the growth potentials of developing countries. Economic growth has been the main driver of poverty reduction mainly relying on burning fossil fuels emitting enormous volumes of $\mathrm{CO}_{2}$. Delinking economic growth and carbon emissions in developing countries is vital to achieving climate stabilization targets. Can developing countries pursue a low carbon development path without compromising efforts in poverty reduction? In this regard, we offer a step in this direction by presenting four different but related papers with a particular focus on developing countries. For the first paper, we investigate whether there is a de-linking between carbon emissions and household affluence in the Philippines. We look into how consumption patterns change as households get richer. The Philippines is of particular interest because its emissions level has been rapidly increasing in the last three decades. In 2010, the 
Philippines ranked $22^{\text {nd }}$ among 144 developing countries in its total carbon emissions with China topping the list followed by India and Russia (IEA 2014). Although the aggregate emissions in the Philippines is relatively low when compared to China, India or Russia, this situation may worsen in the near future because the strong economic growth in the Philippines is largely driven by private consumption according to the Asian Development Bank [ADB] (2014). In fact, private consumption grew by $5.6 \%$ in 2013 and contributed to more than half of the increase in GDP (ADB 2014). Consumer spending is likely to increase in the coming years and will have a significant impact on the aggregate level of carbon emissions. Hence, the first paper investigates household carbon emissions in the Philippines and evaluates current consumption before its emissions can get worse. This is complemented by the second paper, which focuses on decomposing the changes in household emissions into per capita income, carbon intensity and energy intensity. For the third paper, we examine how unequal households are in their emission levels. Emission inequality is of policy relevance because of its distributional implication in reducing household carbon emissions. While the focus of the first three papers is on the household level, the fourth paper takes on a macro perspective and attempts to investigate what motivates developing countries to diversify sources of nonhydro renewable energy. Renewable energy appears to be an efficient and effective option for sustainable development. It can help fuel growth in developing countries without further aggravating the alarming concentration of carbon emissions in the atmosphere.

The results from our empirical investigations show that consumption from fuel and light followed by transportation are the most carbon intensive goods consumed by households while nondurable goods are the least carbon intensive. After controlling for household characteristics, the analyses reveal that income has a significant nonlinear relationship with carbon emissions depicting an inverted U-shaped. This means that emissions increase with income until it reaches its maximum point, after which a further increase in income will lead to a decline in emissions. This reflects what is hypothesized by the Environmental Kuznets Curve (EKC) (Stern 2004). The EKC hypothesizes that at early stages of development environmental pollution will increase, yet beyond some income level economic growth leads to improvement in the environment ${ }^{1}$. However, when using an asset index as a proxy for households' economic status, no turning point is observed and emissions increase as households accumulate more assets. This finding casts doubt on the relevance of EKC hypothesis at the household level. Although our analysis using household income reveals a turning point, it cannot also be taken seriously because the turning point is way beyond the current income levels. Household emissions are strongly increasing within the current income range and still have huge room to increase before reaching the turning point. This implies that the delinking between emissions and income is hardly evident at the household level. In addition, we find that household characteristics such as age and gender of household head, household size and marital status, rural-urban location, education, size of dwelling place and other relevant characteristics significantly matter in explaining carbon emissions.

\footnotetext{
${ }^{1}$ The EKC concept first emerged with the study of Grossman and Krueger (1991) on environmental impacts of North American Free Trade Agreement.
} 
While the findings of the first paper point out the strong correlation between emissions and income on average, decomposing the changes in household emissions into Kaya factors reveals that such correlation varies across the household distributions. Among poor households, the increase in household emissions is largely driven by the income effect while among the rich households the increase in emissions is largely driven by the energy intensity effect through consumption of energy intensive goods. This demonstrates that household emissions can be controlled through reducing energy intensity. This can be done by reducing consumption on energy intensive goods and promoting consumption on energy efficient goods like energy efficient household appliances and light bulbs, more fuel efficient cars or using efficient public transport. By reducing energy intensity, households' aggregate carbon emissions especially the rich households' emissions will eventually decrease. Moreover, we found that the increase in household emissions, which is driven by changes in per capita income and energy intensity, is evenly offset by the change in carbon intensity. This suggests that households are shifting towards a cleaner source of energy as income increases.

Concerning the distribution, we analyze household carbon emissions by examining how unequal the poor and rich households are in their emissions. Mitigation policies aimed at reducing carbon emissions have a different effect in a more equal society than in a more unequal one. In fact, the huge emission inequality between developed and developing countries has been one of the biggest challenges hampering the process of forging international agreements towards reducing global carbon emissions (Heil and Wodon 1997; Clarke-Sather et al. 2011). Our empirical analysis reveals that there is a huge and worsening level of emission inequality between rich and poor households. Among poor households, emissions are more unequally distributed than income while among rich households, emissions are more equally distributed than income. The decomposition analysis highlights the strong influence of income on emission inequality and in addition, emission inequality is mainly driven by energy intensive consumption like fuel, light and transportation. Although more urgent and bold steps have to be taken in order to address income inequality in the Philippines, policy makers should nevertheless take into account the rising carbon emission inequality. This carries important implications in mitigating climate change especially in curtailing overall household carbon emissions.

While household consumption is a matter of private choice and imposing restrictions on what households will consume is rather undesirable, it is nevertheless not meaningless to raise households' awareness about consumption choices that are carbon intensive. If we compare the level of Philippine households' carbon emissions to those in the developed countries, the level is still relatively low. However, as more households are stepping up the economic ladder, they are more likely to lead a carbon intensive lifestyle as manifested by the strong correlation between income and emissions. In this regard, policy makers should devise policies aiming to delink emissions from income without compromising efforts in poverty reduction. With the Philippines' population close to 100 million, where a quarter of it lives below the poverty line (ADB 2014), increases in income will translate current consumption pattern into an enormous increase in carbon emissions. Hence, clean and energy efficient production of goods and services is highly desired. In this regard, fueling economic activities with renewable energy 
appears to be one of the most efficient and effective approaches for clean and sustainable energy development in the Philippines.

Despite the increasing attention on renewable energy, still there is limited empirical analysis about its determinants especially in developing countries. Hence, we fill this gap in the literature by investigating what drives developing countries to integrate renewable energy into their energy system. Specifically, we focus on what motivates developing countries to diversify sources of nonhydro (solar, wind, geothermal, biomass and waste) renewable energy. We use a large data set covering 117 developing countries between 1980 and 2011. Aside from the number of nonhydro sources, we also develop an index use to measure diversification. We explore several estimation techniques such as the negative binomial regression, two-part model and Poisson pseudo-maximum likelihood estimation. Results suggest that higher per capita income, implementation of renewable energy policies, advances in technological innovations, access to finance and improvements in human capital promote the diversification of nonhydro sources of renewable energy. We also document a U-shaped kind of relationship between income and diversification suggesting that at initial stage of development, use of renewable energy will decline but eventually will increase again as the society prospers. Similarly, a high dependency on foreign sources of fuel, increasing world market prices for crude oil, and an increasing population size will push developing countries to diversify sources of nonhydro renewable energy. In contrast, local abundance of hydropower and fossil fuels, aid and foreign direct investment do not contribute to the diversification. Developing countries endowed with coastal areas are more likely to diversify compared to landlocked countries. Finally, we find a strong effect of the Kyoto Protocol on the diversification of nonhydro sources of renewable energy. This is mainly driven by the increasing number of emission-reduction projects implemented under the clean development mechanisms of the Kyoto Protocol.

There is high optimism with the Philippine economy. Despite natural disasters, political and financial shocks, the Philippine economy displayed its resilience and still projects a strong economic performance. Robust private consumption and investment drove economic growth higher in 2013 and strong growth is expected to continue according to ADB (2014). The optimistic projection of Ward (2012) highlights the striking rise of the Philippines, predicting that the country will become the $16^{\text {th }}$ largest economy by 2050 . It will become one of the fastest growing economies in Southeast Asia and this will be accompanied by a growing energy demand with its large population. At present, the country is largely dependent on imported fossil fuels. Thus, harnessing energy from local renewable sources is an important strategy in decreasing dependency on imported and carbon intensive fuels. Currently, it is estimated that renewable energy in the Philippines will grow at an average of $2.4 \%$ a year and is projected to provide $40 \%$ of the country's primary energy needs (Department of Energy, Philippines 2014). Given this information, there is a huge potential for the Philippine economy to shift towards renewable energy thereby avoiding a lock-in on conventional sources of fuel. This will require strategic government intervention to further support wider adoption of renewable energy in the Philippines. If this can be realized, then it will also contribute to the reduction of household carbon emissions. 


\section{Essay 1}

Do Philippine households lead a carbon intensive lifestyle? 


\title{
Essay 1: Do Philippine households lead a carbon intensive lifestyle?
}

\begin{abstract}
Analyses of the level and determinants of household carbon emissions in developed countries are abundant in the literature but very limited in developing countries. Hence, this paper presents an estimation of Philippine households' carbon emissions embodied in the consumption of various goods and services and investigates its determinants. We derive total household carbon emissions by combining input-output analysis with household expenditure for 2000 and 2006. Our estimation shows that household consumption related to fuel, light and transportation are the most carbon intensive goods while nondurable goods are the least carbon intensive. After controlling for household characteristics, the analyses reveal that income has a significant nonlinear relationship with carbon emission depicting an inverted Ushaped. However, when using an asset index as proxy for households' economic status, no turning point is observed and emissions increase as households accumulate more assets. This is further supported by quintile estimates showing that there is a huge disparity in emissions between poor and rich households. Unless consumption patterns change, it is likely that there will be further increases in carbon emissions as households get richer.
\end{abstract}




\subsection{Introduction}

According to Girod and De Haan (2010), households exert a strong influence on the surging increase of greenhouse gas emissions and their consumption behavior is of particular interest in evaluating climate policy options and future emission paths. In the US, more than $80 \%$ of the energy used and $\mathrm{CO}_{2}$ emitted are a consequence of consumer demands (Bin and Dowlatabadi 2005) and in the UK, households contribute substantially to total emissions around 70\% (Baiocchi et al. 2010). Information on household carbon emissions is relatively abundant for most of the developed countries (see for example Lenzen, 1998; Bin and Dowlatabadi, 2005; Druckman and Jackson, 2009; Kerkhof et al., 2009) but information on household emissions from developing countries is mostly overlooked in the literature. Studies coming from developing countries are usually from the major emitters like China and India (Parikh et al., 1997; Zheng et al. 2010). Hence, we pay attention to other developing countries, particularly the Philippines, and investigate household emissions from consuming various goods and services. The Philippines is of particular interest because its emission level has been increasing rapidly in the last three decades. Data from International Energy Agency (IEA) shows that from 1980 to 2010, its aggregate emissions experienced a $130 \%$ increase. In 2010, the Philippines ranked $22^{\text {nd }}$ among 144 developing countries in its total carbon emissions with China topping the list followed by India and Russia (IEA 2014).

Households' consumption has both direct and indirect implications on carbon emissions. Direct emissions come from consumption of household energy such as gas, electricity, petroleum products, coal and biomass while indirect emissions come from energy inputs used in the production of other household goods and services (Druckman and Jackson 2009). If a household decides to buy a refrigerator, direct emission comes from the energy associated with using the refrigerator and indirect emission comes from all inputs used in the production of the refrigerator. We use the framework of an input-output analysis and trace the associated carbon emissions of household consumption down to its intermediates. The method of inputoutput analysis has been used quite often in accounting for the embodied emissions in household consumption (Parikh et al. 1997; Lenzen 1998a; Bin and Dowlatabadi 2005; Kok et al. 2006; Baiocchi et al. 2010). However, this approach is not immune to criticisms. In a paper by Baiocchi et al. (2010), the authors outlined some salient grounds where the estimation of carbon emissions using input-output can be challenged such as the treatment of imported goods being assumed of having similar emission intensities with domestic goods and the characterization of lifestyle through expenditure and not on what people really do. Also Büchs and Schnepf (2013) mentioned that input-output analysis does not reflect product quality and translates expensive goods into higher emissions figure which may have been produced cleanly. However, due to lack of other good alternatives, researchers still rely on this method as it has been widely accepted tool in estimating carbon emissions from household consumption. In this paper, we focus mainly on $\mathrm{CO}_{2}$ emissions associated with household consumption and disregard other green house gases such as methane, nitrous oxide and emissions from land use or deforestation. Carbon emissions comprise the majority of anthropogenic green house gases, being at about $77 \%$ of the world total (Baumert et al. 2005). 
This paper highlights four different points. First, this paper provides an estimation of embodied carbon emissions from household consumption. To the best of our knowledge, this is the first time this exercise has been applied to the Philippines. The aggregate emissions in the Philippines is relatively low compared to industrialized countries. For example in 2010, the per capita emission in the Philippines amount to 0.82 tons of $\mathrm{CO}_{2}$ per capita while for the Organization for Economic Co-operation and Development (OECD) countries the average emission is 10.14 tons of $\mathrm{CO}_{2}$ per capita (IEA 2014). Hence, this paper is in a good position to do an evaluation of household consumption before its emissions can get worse. This will have direct implications on devising polices in mitigating climate change and projecting future household consumption paths. Second, we document which economic sectors and household consumption items are carbon intensive. This will aid in targeting specific consumption goods that are carbon intensive. Third, this paper provides a parametric estimation of whether there is a delinking between household income and emissions. How does the rising affluence of households influence carbon emissions? Lastly, we examine the influence of relevant socio-demographic household characteristics on carbon emissions. Household characteristics other than income play an important role in explaining rising household emissions. This will provide baseline information that is needed to evaluate future projections of emissions as well as to inform policy makers of how to reduce such emissions.

\subsection{Literature Review}

Living means consuming, and consuming requires producing consumer items which causes the depletion of non-renewable energy resources and emissions of greenhouse gases (Lenzen 1998b). By consuming various goods and services, households contribute to the alarming increase in carbon emissions ${ }^{2}$. Hertwich and Peters (2009) quantify greenhouse gas emissions associated with the final consumption for 73 nations and 14 aggregate world regions. They found that $72 \%$ of greenhouse gas emissions are related to household consumption, $10 \%$ to government consumption, and $18 \%$ to investments. On a household level, Girod and De Haan (2010) reported that consumption categories such as living (shelter), car driving, and food consumption all together amount nearly to $70 \%$ of the total greenhouse gas emissions among Swiss households. Also Kenny and Gray (2009) using a model of Irish households found that the average annual household emissions are comprised of $42.2 \%$ home energy use, $35.1 \%$ transport, $20.6 \%$ air travel and other fuel intensive leisure activities. A paper by Parikh et al. (1997) in India showed that rich households consume more carbon intensive products like electricity, transport and use relatively more resources in the form of minerals and metal products.

Lenzen (1998a) used input-output derived carbon intensities in calculating the Australian households' carbon emissions. He found out that most of the greenhouse gas emissions attributable to Australians come from households' consumption of goods and

\footnotetext{
${ }^{2} \mathrm{CO}_{2}$ emissions and carbon emissions are used interchangeably in this paper.
} 
services and that the present increases in emission are strongly correlated to income growth. Kerkhof et al. (2009b) evaluated the relationships between expenditures and the environmental impact of climate change by combining household expenditures with an environmentally extended input-output analysis using data from the Netherlands. They found that the environmental impact arising from consumption of goods and services increases with household expenditures. Several other studies have analyzed the effect of income on household emissions (Lenzen et al. 2006; Weber and Matthews 2008; Girod and De Haan 2010; Büchs and Schnepf 2013; Ala-Mantila et al. 2014). All of these studies confirmed a positive relationship between income and household emissions but with varying elasticities across countries. For example, a $1 \%$ increase in income is associated with $0.70 \%$ increase in emissions in the US (Weber and Matthews 2008), 0.94\% in Switzerland (Girod and De Haan 2010), $0.43 \%$ in the UK and $0.79 \%$ in Finland (Ala-Mantila et al. 2014).

The majority of the available studies on household emissions come from developed countries while estimations of household emissions from developing countries are very limited. Hence, we fill this gap in the literature by bringing developing countries, particularly the Philippines, into perspective. We are especially interested in quantifying how elastic the change in household emissions is with respect to changes in household income. As of writing, this is the first study that attempts to estimate household emissions and investigate their determinants in the Philippines' setting. In addition, we also examine the influence of household characteristics on carbon emission. Baiocchi et al. (2010) criticized that most input-output based lifestyle studies on household carbon emissions are purely descriptive in nature and emphasized the importance of establishing a link between emissions and households' socio-demographic factors. This is supported by Büchs and Schnepf (2013) who found out that household characteristics play a significant role in explaining household emissions in the UK. Taking this into consideration, we examine the influence of household characteristics on carbon emissions as household characteristics can have a distributional implication in devising policies towards mitigating climate change.

\subsection{Theoretical background}

This paper is partly grounded on the concept of Environmental Kuznets Curve (EKC). The EKC concept first emerged in a study by Grossman and Krueger (1991) focusing on the environmental impacts of the North American Free Trade Agreement. The EKC was named after Kuznets (1955) for his work on income inequality and economic development. The EKC hypothesis proposes an inverted U-shaped relationship between per capita income and environmental degradation. For a given society, environmental pressures or pollutions are expected to increase in the early stages of growth but eventually they will reach their peak and then start to decline after income exceeds a certain level. This decline in environmental pressures can be attributed to changes in production structure, greater environmental awareness, and the use of environmental or climate friendly technologies. If EKC hypothesis is true, then rather being a threat to the environment, economic growth will eventually lead to 
environmental improvement (Stern 2004). Similarly, we use the EKC concept in investigating household emission. Households exert environmental pressure by their consumption choices. As household income increases, their ability to change consumption patterns improves (Heerink et al. 2001). For example, according to Cropper and Griffiths (1994) as cited by Heerink et al. (2001) it can be hypothesized that rising incomes may first increase demand for fuelwood but at higher income level, fuelwood will be increasingly replaced by modern sources of energy. This demonstrates that the general argument outlined by the EKC can also be valid at the household level. We proxy environmental pressure with the aggregate household carbon emission and investigate whether higher household income will translate to greater environmental concern. This hypothesized concave relationship between household income and carbon emission is illustrated graphically in Figure 1.1.

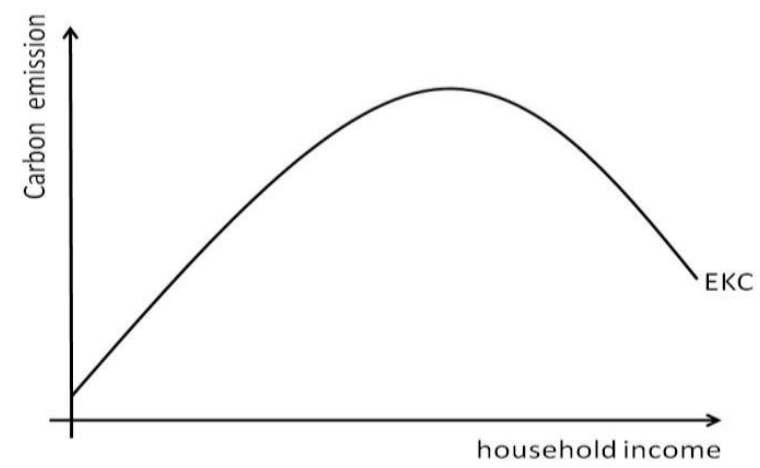

Figure 1.1. Environmental Kuznets curve for household carbon emission and income.

Several studies have empirically investigated the presence of EKC however, evidence is still a matter of contention. Dinda (2004) provides a comprehensive survey of empirical studies on EKC and found that only in local pollutants EKC is evident but not with carbon emission and there is no agreement in the literature about the income level at which environmental degradation starts to improve. Stern (2004) argued that the empirical evidence of EKC is not robust and showed that there is little evidence for a common inverted U-shaped pathway that countries follow as economy progresses. Yaguchi et al. (2007) also found out in a comparative study between China and Japan that EKC hypothesis does not hold true with $\mathrm{CO}_{2}$ emissions but only evident with sulfur dioxide $\left(\mathrm{SO}_{2}\right)$ emissions.

Although EKC has been mainly explored at the macro level, several studies have also investigated the presence of EKC at the household level. Kahn (1998) shows that there exists a household level EKC using household vehicle emission. However, among Scottish households, Cox et al. (2012) found no evidence of EKC on household transport emission and richer households own more and newer vehicles but they do not own less polluting vehicles suggesting that richer household do not choose to internalize environmental cost. Lenzen et al. (2006) did a comparative analysis among households in Australia, Brazil, Denmark, India and Japan and tried to search for evidence on the environmental Kuznets Curve (EKC) at the household level. However, results show that the data does not support the Kuznets curve. Household energy requirements increase monotonically with household expenditure and no 
turning point was observed (Lenzen et al. 2006). Golley and Meng (2012) also found no evidence on EKC among Chinese urban households. They concluded that it is likely that there will be no turning point for household emissions but rather it will increase with income.

Though there is no conclusive evidence of the presence of EKC both at the macro and micro level, the EKC hypothesis is still widely used in analyzing how economic growth or in the case for households, how improvement in the lifestyles affects the environment. However, Büchs and Schnepf (2013) found that household characteristics other than income are highly relevant factors in explaining the variations in household emissions. Hence, we consider several household socio-demographic factors such as urban-rural location, education, household size, age, dwelling place, gender and marital status that can play an important role in explaining household emissions.

\subsection{Methodology}

Kok et al. (2006) highlighted three different methods using input-output analysis in estimating embodied energy or emissions, namely: the basic, expenditure and process approach. The basic approach uses national accounts, the expenditure approach uses data from household consumption and the process approach determines the emissions generated through the lifecycle of a product starting from production through to disposal. For our paper, we used the expenditure approach in accounting for the embedded carbon emissions from households' consumption. By using this approach, we were able to match household consumption items with the sectors that produced them. This accounts for carbon emission based on what the households consume. This method has been widely used in the literature (Parikh et al.1997; Pachauri and Spreng 2002; Lenzen et al. 2006; Kerkhof et al. 2009; Baiocchi et al. 2010).

The figure below shows the process flow of combining the input-output table and expenditure survey in deriving the carbon emissions of goods and services. It shows that the emission intensities of sectors, which is needed in matching with household expenditure survey, was derived using the input-output analysis tracing the amount of energy used and its corresponding carbon emission in the production of goods and services.

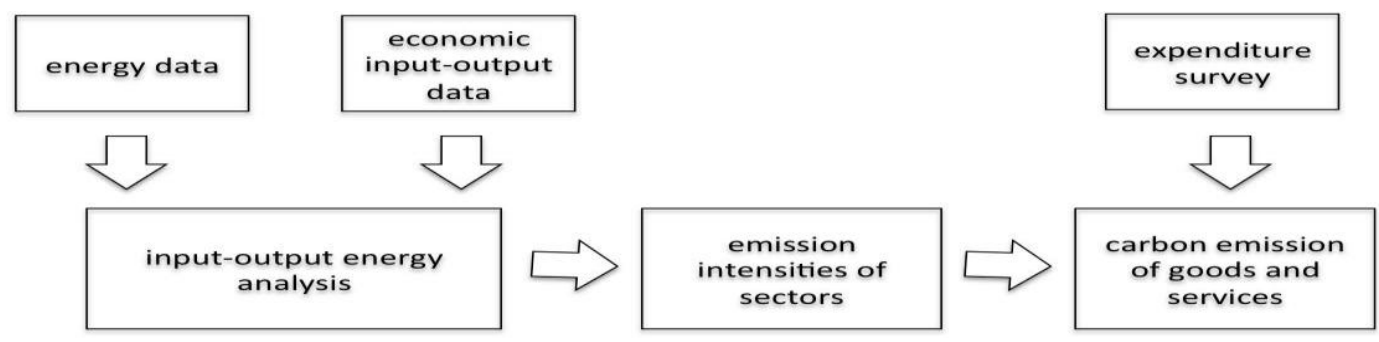

Source: Kok et al. (2006)

Figure 1.2. Process flow using the expenditure approach in estimating household carbon emissions. 


\subsubsection{Estimation of households' carbon emissions}

The method of input-output analysis was first developed by Leontief in 1941 when he studied the relationships between economic sectors. Many authors have explored the mechanisms of the input-output analysis and extended it to investigate environmental issues. Minx et al. (2009) provides a comprehensive literature review on studies using the inputoutput analysis in estimating carbon emissions. The basic structure of input-output analysis is given as follows:

$$
X=(I-A)^{-1} Y
$$

where $X$ is the vector of total output, $A$ is the technical coefficient matrix or direct requirement coefficients $^{3}$ which relate to the output $x_{j}$ of industry $j$ to its inputs from industries $i, I$ is the unit matrix, and $Y$ is the vector of final demand. Equation 1.1 is the fundamental representation of the input-output analysis and the $(I-A)^{-1}$ matrix is well known as the Leontief inverse matrix. Correspondingly, the carbon emission intensity $(C I)$ of each economic sector can be computed as follows:

$$
C I=c^{\prime}(I-A)^{-1} y
$$

where $c$ is a vector containing carbon emission coefficient for the energy commodity $i$ used by sector $j$ in a given country. This was taken from the Global Trade Analysis Project (GTAP) (Lee 2008). The vector $c$ is a product of several factors including fuel consumption of energy commodities by sectors, the conversion coefficient of energy commodities, the ratio of carbon stored in the energy commodities used by sectors, the emission factor of energy commodities and the fraction of carbon oxidized of energy commodities. The derivations revolve around the transformation of energy commodities used in the productions to carbon emissions. The energy commodities included in deriving carbon emissions include coal, crude oil, natural gas, petroleum products, electricity and gas. The method used in estimating $\mathrm{CO}_{2}$ emissions follows the procedure suggested in the revised 1996 IPCC Guidelines (IPCC 2013b).

Mapping of the sectors has to be done for the consistent linking of the different data sources. The carbon emissions coefficients were coming from 57 sectors while the inputoutput table is a 240x240 matrix. We follow the disaggregation method available in GTAP in disaggregating 57 sectors to 240 sectors using concordances so that it matches with the sectors of Philippines IO table. These concordances used standard classifications and are available online (GTAP 2011). For example, vegetables, fruits and nuts which are represented by 1 sector in the GTAP is to be matched with 6 different sectors in Philippine IO table including leafy and steam vegetables, banana, pineapple, mango, citrus, fruits and nuts sectors.

\footnotetext{
${ }^{3}$ The technical coefficients or direct requirement matrix presents the unit cost structure of production in an economy. This describes the coefficient value of intermediate inputs and primary inputs required in the production of one unit of output of the industry. This is derived by dividing each element in the intermediate transactions matrix by the total input of each sector (NSCB 2014).
} 
Appendix Table 1 provides the detailed sectoral mapping of the GTAP sectors with the Philippines IO table.

Solving carbon emission intensity using equation 1.2 yields $240 \mathrm{CO}_{2}$ emission intensities measured in tons of $\mathrm{CO}_{2}$ per thousand Philippine pesos. Appendix Table 2 provides the list of 240 sectors with their corresponding carbon emission intensity. We matched these 240 sectoral carbon emission intensities with close to 200 household consumption items. Then, the carbon emission of each household consumption category was calculated by multiplying (i) the $\mathrm{CO}_{2}$ emission intensity of each economic sector $(C I)$ and (ii) the corresponding household expenditure category (cons). Summing up all the carbon emissions of each consumption category yields the total household carbon emissions $\left(h h C \mathrm{O}_{2 i}\right)$, that is,

$$
h h C O_{2 i}=\Sigma_{i}^{j}\left(C I * \operatorname{cons}^{h h}{ }_{i j}\right)
$$

where $\operatorname{cons}^{h h}$ represents household consumption items, $i$ is the individual household and $j$ is the expenditure category. The household carbon emissions are measured in tons of $\mathrm{CO}_{2}$ and dividing it with household size yields the per capita carbon emission.

In summary, Figure 1.3 provides the estimation procedure and the matching of IO sectors with household consumption. Through this method, we can estimate in detail the embedded carbon emissions of every household consumption item. We match the 274 derived carbon intensities with around 200 consumption items. Since we are matching household consumption items with economic sectors, there are several household items produced within the same sector carrying the same carbon intensity. However, there are some sectors in the IO table left unmatched because they have no direct household consumption match. For example, sectors like blast furnace, asphalt manufacturing, public administration and defense do not have a direct match with the items listed in the household consumption. These sectors were left unmatched and were not used in the estimation of household carbon emissions. Because of these unmatched sectors, we expect that the estimated household emissions from consumption will be relatively lower than the estimation using the production side. Appendix Table 3 provides the matching of household consumption items with IO sectors.

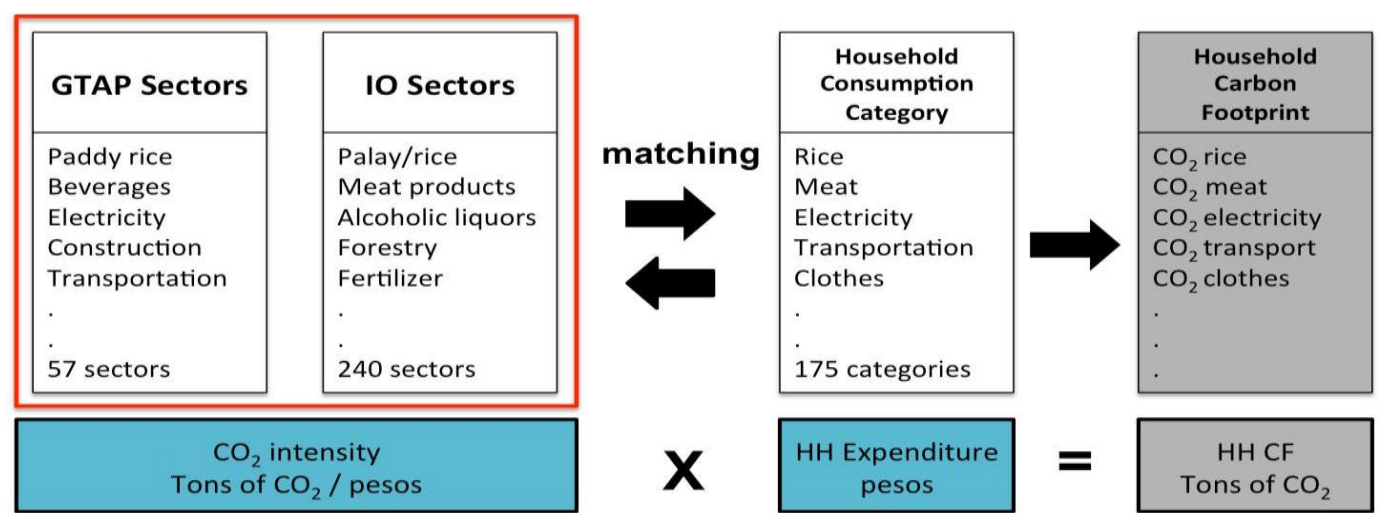

Figure 1.3. Process flow estimation and matching of IO sectors with household consumption. 


\subsubsection{Data and limitation}

To carry out the estimation of household carbon emissions, we need three data sets. First, the Philippine Input-Output (IO) table for year 2000 acquired from the National Statistical Coordination Board (NSCB). The 2000 IO table is a matrix of 240x240 industrial sectors. It is the $9^{\text {th }}$ series of the inter-industry study of the Philippine economy since the first benchmark IO table in 1961. We use the latest publicly available IO table in the Philippines. Second, we need the Global Trade Analysis Project (GTAP)'s carbon emission coefficient (Lee 2008). This carbon emission coefficient from GTAP is a $1 \times 57$ vector representing 57 aggregated sectors. Third, we need data on households' consumption. For this, we use the Family Income and Expenditure Survey (FIES) of the National Statistics Office (NSO). The FIES is the main source of income and expenditure data among households in the Philippines (Ericta and Fabian 2009). It is a nationwide survey conducted every three years by the NSO. To match our IO table, we use FIES data from 2000. The FIES include around 200 disaggregated household consumption categories. The household survey in 2000 has 37,766 households while in 2006 the sample size is 38,483 . Due to data limitations, we use the carbon intensity for year 2000 to compute household carbon emission in $2006^{4}$. We use the consumer price index to deflate the expenditure in 2006 to make it comparable with household expenditure in 2000.

While this is the first study to evaluate Philippine household carbon emission, our estimation is limited in several ways. The first limitation is on the estimation of household emissions. We did several aggregations and disaggregations in the computation and in the process we might have lost some important information. For example, the GTAP emission coefficients from 57 sectors were disaggregated into 240 IO sectors and in addition, we aggregated several expenditure categories to represent major household consumption. Second is the treatment of imported goods. We assume that imported goods have the same carbon intensity as locally produced goods. By assuming this, we are somehow overestimating household carbon emissions because goods coming from developed countries have lower carbon intensities than the domestic production but there are also imported goods coming from countries with high carbon intensities, and in this case we might be underestimating household emissions. A proposed method to deal with this problem is using a multiregional input-output model (Weber and Matthews 2008; Minx et al. 2009). If we apply the multiregional input-output model, another hindrance would be the matching of household items because we do not have information in the household survey about imported goods. A third limitation is on converting expenditure to emissions. According to Büchs and Schnepf (2013) expenditure does not always necessarily equate to the consumption on which emissions is based. For instance, an expensive bread may have lower emissions in comparison to a cheap one, but expenditure translates the expensive bread as having higher emissions. This in turn would bias the emissions of the rich who can afford to buy quality goods, which may have been cleanly and efficiently produced but are expensive. Unfortunately, with the

\footnotetext{
${ }^{4}$ We assume that there is no strategic shift in production structure towards a cleaner and efficient production and further assume that carbon intensity is similar for this time period.
} 
input-output analysis we cannot distinguish product quality. We only have information on how carbon intensive the sectors are but we do not have information about the product quality in that sector. A potential way to resolve this problem is to incorporate product quality in the estimation by doing a detailed life cycle analysis for every household consumption item. However based on our knowledge of the recent literature, we can hardly find any household studies taking into account product quality of goods consumed by households. Due to practicality and lack of other good alternatives, estimating emissions through combining the input-output and expenditure approach is still widely used in the literature (Parikh et al. 1997; Lenzen 1998a; Weber and Perrels 2000; Pachauri and Spreng 2002; Bin and Dowlatabadi 2005; Kerkhof et al. 2009; Baiocchi et al. 2010; Büchs and Schnepf 2013).

\subsubsection{Determinants of household carbon emissions}

One of our main concerns is to evaluate how households' carbon emissions are influenced as households become more affluent. While household income is likely to be the main determinant, other household characteristics play an important role in explaining household carbon emissions. To evaluate this relationship, the following regression model is postulated as follows:

$$
\ln \left(h h C O_{2 i}\right)=\alpha+\beta_{1} \ln \left(i n c_{i}\right)+\beta_{1} \ln \left(i n c_{i}\right)^{2}+\gamma_{i} X_{i}+\varepsilon_{i}
$$

where $\ln \left(h h C O_{2 i}\right)$ is the $\log$ of household carbon emission, $\ln (\operatorname{inc})$ is the $\log$ of household income or expenditures with squared term included to evaluate for the presence of EKC or nonlinearity at the household level,$X$ is a vector of control variables capturing household characteristics and $\varepsilon_{i}$ is the usual disturbance term. Socio-demographic characteristics account for an important aspect of households' lifestyle and consumption preference. Several studies have found that household characteristics other than income are relevant for household emissions (Lenzen et al. 2006; Baiocchi et al. 2010; Büchs and Schnepf 2013). Based on this evidence, we include several relevant socio-demographic characteristics that help explain household emissions. The control variables are age and sex of household head, marital status, level of education, household size, location whether in rural or urban areas, access to electricity, size of the dwelling place, regions where the households are located, and others household characteristics. Since we used two periods of household survey, we run a pooled regression analysis with a year dummy included in the control.

Age and household size can influence total household emission. Younger households have different consumption patterns than older households. Bigger household size entails more consumption, however it's also possible that household members share resources thereby using resources efficiently. Higher education increases awareness on environmental issues so we expect that emission and education be negatively correlated. However, it is also plausible that education is positively correlated with emission if gaining higher education is associated with a lifestyle that is carbon intensive. Lenzen et al. (2006) found conflicting 
results on the association between emissions and education. In Australia, they found negative association between emissions and education but in Brazil and India they found positive association arguing that higher education in developing countries is usually a privilege for the rich. Urban-rural setting affects household emissions differently. In most developed countries, living in a rural area is associated with higher carbon emissions because of greater car dependency and higher transport cost. However, the Philippines may present a different case and it is likely that rural area will be less carbon intensive because they consume less energy as compared to urban households. Access to electricity and larger dwelling place are both assumed to be positively associated with household emission. Having access to electricity will increase emissions due to electricity consumption and also emissions from electricity dependent durable goods. Similarly, big houses translate to larger cost in repair and maintenance. While most studies do not include sex of the household head in the analysis, we consider this as a relevant variable capturing gender effect on consumption. It is quite known that men and women have different consumption preferences. Hence, it is interesting to see how gender affects carbon emissions. Households' marital status may influence carbon emissions because married couples have different lifestyle as compared to singles. Lastly, we also control for geographic differences and time variation by including regional and year dummy.

The main coefficient of interest is $\beta_{l}$. It captures how carbon emissions change as household income changes. Based on previous studies, carbon emissions will increase as household income increases, however aside from the sign of the coefficient, we are particularly interested in the magnitude of the coefficient and check whether the elasticity is significantly different from 1 . Since we model a log-log regression, $\beta_{1}$ measures how elastic is the change in emissions with respect to the change in income. To further analyze the effect of rising income on household carbon emissions, we replace the income variable with income quintiles. We divide the households into five income groups and look into detail how carbon emissions behave across quintiles. We proceed as follows:

$$
\ln \left(h h C O_{2 i}\right)=\alpha+\beta_{1} Q_{\text {uint }} 1_{i}+\ldots+\beta_{5} Q_{\text {uint }} 5_{i}+\gamma_{i} X_{i}+\varepsilon_{i}
$$

where Quint1 is a dummy variable capturing the poorest $20 \%$ of the households, Quint5 captures the richest $20 \%$ of the households, $X$ is a vector of control variables and $\varepsilon_{i}$ is the error term. Since income is potentially correlated with some of the household characteristics, we tried to control for this by applying two-stage estimation. First, we regress household carbon emissions with only income quintiles then in the second stage, we collect the predicted residuals from the previous analysis and run the regression with household characteristics on the independent side and the residuals as the dependent variable.

For all of the specifications, we model the relationship between household emission and income. We assume that income is exogenous and that reverse causality may not work in this case because household emission is a consequence of income. To reduce omitted variable problems, we include in the analysis various relevant household characteristics to capture as many observable characteristics influencing emission as possible. However, still we cannot 
discount the fact that there might be other unobservable household traits influencing emission. We also pay attention to the issue of multicollinearity since we are controlling for several household characteristics. We check it by computing the Spearman correlation coefficient among explanatory variables. Results are not problematic and most of the correlations are relatively weak $(\rho<=0.2)$ with some exception such as the correlation between male and being married $(\rho=0.5)$. In addition, after each regression we further check the severity of multicollinearity using variance inflation factors (VIF). By convention, VIF values greater than 10 are problematic, however in all specifications the VIF values ranges only from 3 to 7. Hence, we are confident that multicollinearity may not be an issue in this case.

\subsubsection{Asset index construction}

Income and expenditure data are interchangeably used to reflect households' economic profile. However, many studies are inclined to use expenditure rather than income because expenditure data is more reliably reported and more stable than income (Klasen 1997). Hentschel and Lanjouw (1996) stated that income data is difficult to collect in developing countries especially in rural settings, thus, household expenditure may provide a better proxy for long term economic status (Deaton 1992). However, in this analysis the expenditure variable is endogenous by construction because household emissions were derived as the product of carbon intensity and household expenditure. Thus, we rely on income data. Considering however the arguments above, we consider another measure and proxy households' economic status by constructing an asset index.

We use the method suggested by Filmer and Pritchett $(2001)^{5}$. They used data on household ownership of durable goods, characteristics of household dwellings and land ownership to construct a proxy for household wealth. This method is being used by the World Bank as a way to assess the socio-economic status of households based on asset ownership (Gwatkin et al. 2007). An improvement of the method was proposed by Kolenikov and Angeles (2009) through accounting for discrete data without breaking it into dummy variables. Following Filmer and Pritchett (2001), we construct a linear index from households' asset ownership using the concept of principal component analysis. We classify several household assets into three major categories: (i) household ownership of durable goods (with 14 indicators including ownership of radio, TV, stereo, vtr/dvd player, refrigerator, washing machine, air-condition, phone, oven, computer, living room \& dining set, car, and motorbike); (ii) characteristics of household dwelling (with 9 indicators such as whether the house is made of strong or light materials, kind of toilet either flush toilet or pit/latrine or no toilet at all, sources of water either from the water system, pump/well or from river and access to electricity); and (iii) ownership of house and lot (with 3 indicators including owning the house $\&$ lot, renting the house \& lot or not owning or renting house $\&$ lot). Results of the asset index construction for year 2000 and 2006 are presented in the Appendix Table 4.

\footnotetext{
${ }^{5}$ For more discussion of the methods, readers may refer to the paper of Filmer and Pritchett (2001).
} 


\subsubsection{Income elasticity}

It is then noteworthy to analyze which consumption items households will prioritize as they become richer. We use the concept of elasticity to analyze the percentage change in consumption resulting from a percentage change in household income. We model the elasticity of different consumption catgerories as follows:

$$
w_{i j}=\alpha+\eta_{1} \ln (i n c)_{i}+\gamma_{i j} X_{i}+\varepsilon_{i j}
$$

where $w_{i j}$ represents the share of total income allocated to the $j t h$ consumption category by the $i$ th household, $\ln \left(i n c_{i}\right)$ is the income of household $i$ in logs, $X_{i}$ is a vector with household characteristics and $\varepsilon_{i j}$ is the usual error term. In addition, we split the analysis by location to capture the difference in lifestyle between urban and rural households.

\subsection{Results and Discussion}

\subsubsection{Carbon intensity of economic sectors}

We present the results of our estimation on $\mathrm{CO}_{2}$ emission intensity measured in grams of $\mathrm{CO}_{2}$ per Philippine currency (in peso). This captures the estimated amount of $\mathrm{CO}_{2}$ emitted by different sectors of the economy. Table 1.1 presents the top 20 and lowest 20 carbon emitting sectors. The most carbon intensive sectors are related to energy, travel, mining and manufacturing. The highest carbon-emitting sector of the Philippine economy is chromite mining with a carbon intensity of 158.86 grams of $\mathrm{CO}_{2}$ per peso followed closely by the generation of electricity with a carbon intensity of 157.27 grams of $\mathrm{CO}_{2}$ per peso. Several transport related sectors are also found to be very carbon intensive. Conversely, the least carbon intensive sectors are related to agriculture. Topping the list of lowest carbon emitting sector is the production of other agricultural crops with an estimated intensity of 0.72 grams of $\mathrm{CO}_{2}$ per peso followed by other vegetables and root crops. Most of the least $\mathrm{CO}_{2}$ emission intensive sectors in the economy include the production of agricultural crops, fruits, vegetables, copra, etc. This is plausible because these sectors do not require huge amount of energy to produce compared to manufacturing sectors or transportation sectors. In addition, we do not capture the emissions from land use change and methane emissions from agriculture related production. We use these derived carbon intensities in estimating household carbon emissions by matching them with every consumption item listed in the household expenditure survey for year 2000 and $2006^{6}$.Appendix Table 2 provides the list of all sectors from the input-output table with its corresponding carbon intensity.

\footnotetext{
${ }^{6}$ We use the same carbon intensity for the estimation of household carbon footprint in 2006 . We assume that there was no radical change in the Philippine production structure from 2000 to 2006 . Hence, the same carbon intensity can be used in the estimation of household carbon emissions.
} 


\subsubsection{Characteristics of households' carbon emissions}

Results of our estimation show that on average households emitted 1.46 tons of $\mathrm{CO}_{2}$ in 2000 and it increased to 1.86 tons in 2006. On per capita basis, the average per capita emissions in 2000 amounted to 0.32 tons of $\mathrm{CO}_{2}$ and in 2006, it amounted to 0.44 tons of $\mathrm{CO}_{2}$. Estimates of carbon emissions from the IEA (2014) showed that in 2000, the Philippines emitted 0.87 tons of $\mathrm{CO}_{2}$ per capita and in 2006 it declined to 0.74 tons of $\mathrm{CO}_{2}$ per capita. The decline in emissions is partly attributed to the large increase in population from 77.3 million in 2000 to 87.1 million in 2006 but mainly due to improvement in energy intensity (World Bank 2010). If we compare it with the household level, there was a decline in the average household size from 5.24 members in 2000 to 4.94 members in 2006 (Table 1.2). Our household level estimation of carbon emissions is relatively lower than the estimation provided by IEA (2014) because our focus is only on household consumption and not on all economic activities. We then disaggregate total carbon emissions into twenty major consumption items (Figure 1.4). Results show that emissions from fuel and light followed by transportation are relatively higher than the rest of consumption categories. This is plausible because these household items are energy intensive. Among the food related expenditures, fruits and vegetables have low carbon emissions while meat, dairy and egg posted a relatively higher emissions. The least carbon intensive goods consumed by households are nondurable goods, recreation and communication. This observation is consistent in both years.

Table 1.1. Top 20 highest and lowest emitting $\mathrm{CO}_{2}$ sectors.

\begin{tabular}{|c|c|c|c|c|c|}
\hline \multicolumn{2}{|r|}{ Highest $\mathrm{CO}_{2}$ Emitting Sectors } & \multirow{2}{*}{$\begin{array}{c}\begin{array}{c}\text { emission } \\
\text { intensity } \\
\left(\mathrm{g} \mathrm{CO}_{2} / \text { peso }\right)\end{array} \\
158.8611\end{array}$} & \multicolumn{2}{|r|}{ Lowest $\mathrm{CO}_{2}$ Emitting Sectors } & \multirow{2}{*}{$\begin{array}{c}\begin{array}{c}\text { emission } \\
\text { intensity } \\
\left(\mathrm{g} \mathrm{CO}_{2} / \text { peso }\right)\end{array} \\
0.7242\end{array}$} \\
\hline 1 & Chromite mining & & 1 & Other agricultural crops & \\
\hline 2 & Electricity & 157.2655 & 2 & Other vegetables, root crops & 1.1528 \\
\hline 3 & Structural concrete products & 78.4560 & 3 & Coconut including copra & 1.2173 \\
\hline 4 & Coal mining & 77.9721 & 4 & Ownership of dwellings & 1.4512 \\
\hline 5 & Other non-metallic mineral & 76.0676 & 5 & Wood-working machinery & 1.5920 \\
\hline 6 & Cement manufacture & 72.5321 & 6 & Other fruits and nuts & 1.7225 \\
\hline 7 & Other glass and glass products & 72.0594 & 7 & Corn & 2.0624 \\
\hline 8 & Air transport & 71.1750 & 8 & Mango & 2.2189 \\
\hline 9 & Water & 67.4639 & 9 & Pineapple & 2.2363 \\
\hline 10 & Glass container & 63.8897 & 10 & Radio \& TV receiving sets & 2.5782 \\
\hline 11 & Railway transport & 63.1313 & 11 & Palay & 2.5977 \\
\hline 12 & Structural clay products & 57.9100 & 12 & Parts for radio, TV & 2.6046 \\
\hline 13 & Tour and travel agencies & 57.2530 & 13 & Leafy and stem vegetables & 2.6091 \\
\hline 14 & Other transport agencies & 55.2023 & 14 & Tobacco & 2.6526 \\
\hline 15 & Manufacture of ice & 53.6820 & 15 & Cattle & 2.7612 \\
\hline 16 & Pottery, china, earthenware & 53.4887 & 16 & Semi- conductor devices & 3.0416 \\
\hline 17 & Public utility cars and taxicab & 52.6268 & 17 & Forestry & 3.2852 \\
\hline 18 & Road freight transport & 52.4432 & & Rice and corn milling & 3.3026 \\
\hline 19 & Jeepney, tricycles, others & 52.1624 & 19 & Carabao & 3.3390 \\
\hline & Bus line operation & 51.3768 & & Citrus fruits & 3.3846 \\
\hline
\end{tabular}

Source: Author's computation based on input-output table for 2000.

The full list of 240 IO sectors with their corresponding emission intensity is provided in Appendix Table 2. 


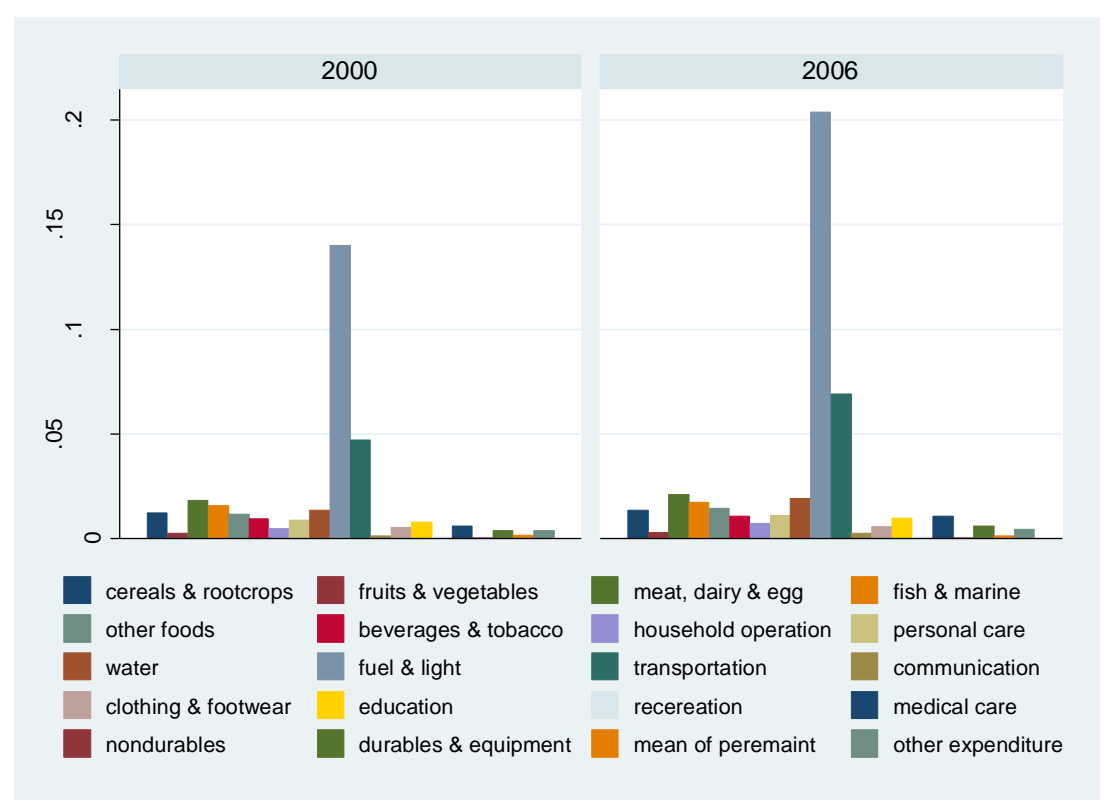

Figure 1.4. Mean per capita $\mathrm{CO}_{2}$ emissions by expenditure categories.

In Figure 1.5, we look at the average emission by income quintile and further disaggregate carbon emissions into major consumption categories. Results show that there is a huge gap in carbon emissions between households from the lowest and highest quintile. In 2000 , households in the poorest quintile (quintile 1) emitted on average 0.10 tons of $\mathrm{CO}_{2}$ per capita while the richest quintile (quintile 5) emitted on average 0.77 tons of $\mathrm{CO}_{2}$ per capita. The rich households are 7.7 times higher in their emissions than poor households. In 2006 we observed an increase in emissions across all income quintiles but the increase in emissions in the richest quintile is more evident. Households in the poorest income quintile emit 0.12 tons of $\mathrm{CO}_{2}$ per capita while households in the richest quintile emit 1.02 tons of $\mathrm{CO}_{2}$ per capita. In 2006, the rich households are 8.5 times higher in emissions than poor households. This means that the emission gap between the rich and poor widened from 7.7 in 2000 to 8.5 in 2006 . Though the emission gap is rising, in contrast we find that the gap in income per capita is declining. In 2000, the income of the rich was 7.9 times higher than the income of the poor while in 2006, the income of the rich was 7.2 times higher than the income of the poor. In short, the emission gap between the rich and poor widens while the income gap between the rich and the poor contracts. We will on elaborate this topic in Essay 3 wherein we focus on emission inequality and find that emission inequality worsens while income inequality improves.

Notably, from the lowest income quintile to the $4^{\text {th }}$ income quintile, we observed a gradual increase in per capita emissions but from the $4^{\text {th }}$ quintile to the $5^{\text {th }}$ quintile we observed a rather huge jump in the level of emissions (Figure 1.5). This is an indication that the rich households are leading a carbon intensive lifestyle. Looking at the major consumption categories, we observed that the emission from fuel \& light and transportation increases dramatically as households' income increases while the emissions from food items do not change that much as households become more affluent. Particularly the emissions attributed 
to cereals, root crops, fruits and vegetables do not vary that much across income quintiles as compared to the emissions from meat and dairy products. Overall, fuel \& light and transportation occupy a larger share of household emissions and the increase in emissions from 2000 to 2006 is mainly driven by the increase in emissions of the richest quintile.

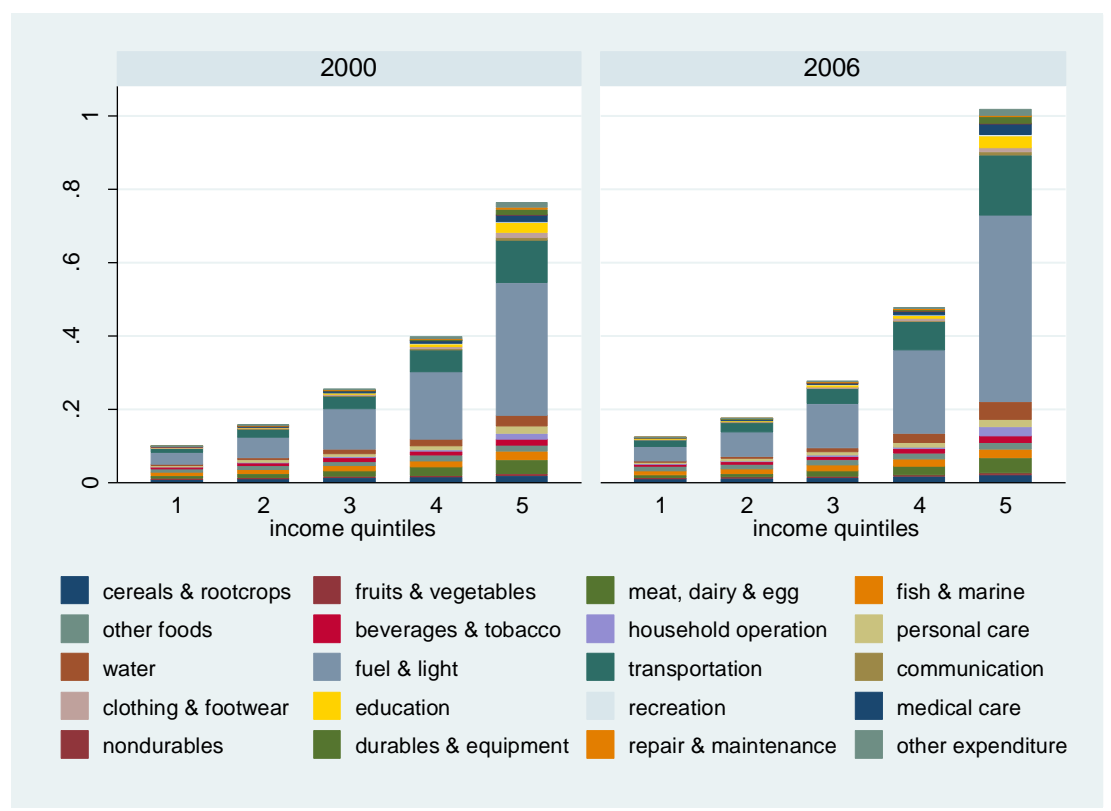

Figure 1.5. Mean per capita $\mathrm{CO}_{2}$ emissions by income group.

Figure 1.6 confirms our assumption earlier that emissions per capita are higher in urban areas than rural areas. On average, the level of emissions in urban areas is more than double the level of emissions from rural areas. This is because there is a significant share of households in the rural areas that consume very little energy. This can be explained by the following circumstances. Electrification rate is lower in rural areas as compared to urban settings. Concerning transportation, households in the urban areas are more dependent on cars while rural households are not. Public transportation is more frequent and available in urban areas than in rural areas. Since public transport in the Philippines is not efficient especially in the urban areas, this resulted to heavy congestions of vehicles and fuel inefficiency. These differences in consumption behavior related to fuel, light and transportation largely explain the disparity in carbon emissions between urban and rural households in the Philippines. However, this situation is different in comparison with households from developed countries. Households in urban areas have lower carbon emissions than rural households. People in urban centers live closer to their work place and the proximity of shopping centers and leisure places require less energy for transportation. Also urban areas have improved public transport systems. Several studies in developed countries have found evidence that carbon emissions are higher among households in rural areas than households in urban areas (Fahmy et al. 2011; Ala-Mantila et al. 2014). 


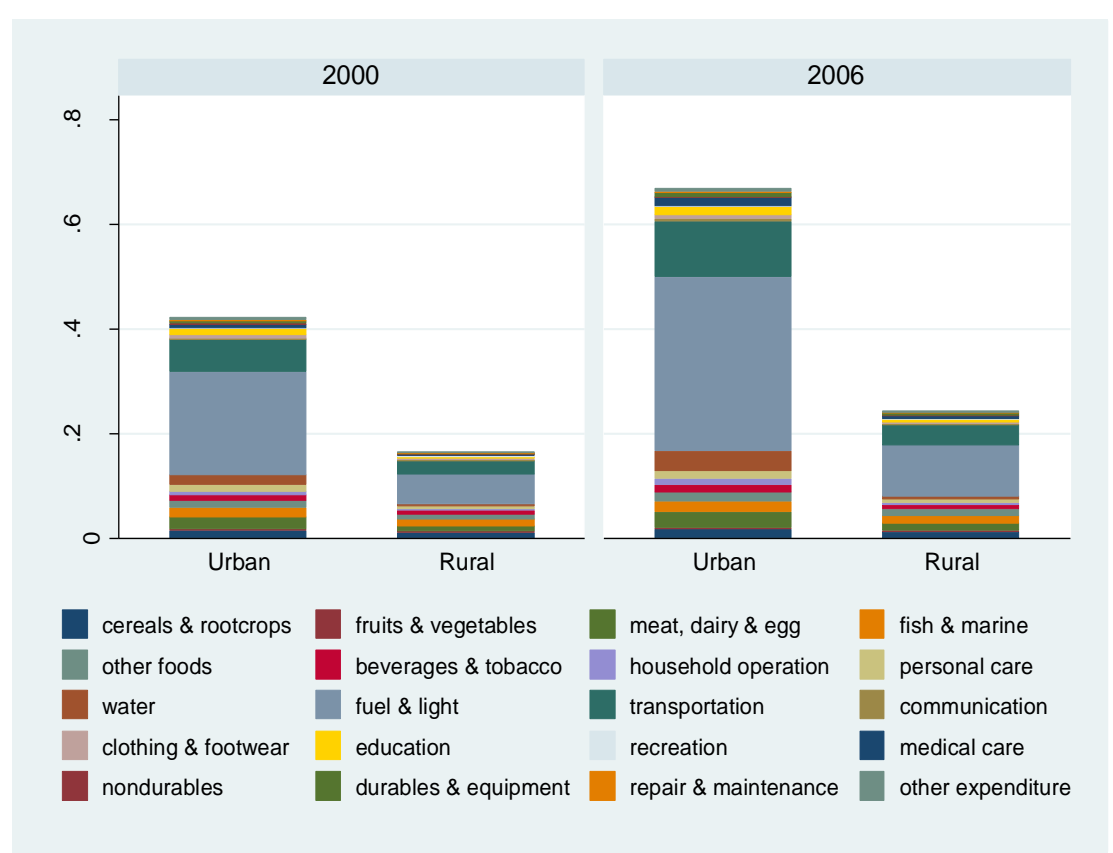

Figure 1.6. Mean per capita $\mathrm{CO}_{2}$ emissions by urban-rural location.

Compared to the households from the US and UK where the average household emissions in 2004 were as much as 48 tons and 21 tons of $\mathrm{CO}_{2}$ respectively (Weber and Matthews 2008; Druckman and Jackson 2009), the Philippine household carbon emissions are undoubtedly way lower. In terms of per capita emissions, the average emissions per capita in the Philippines in 2010 amounted to 0.82 tons, which is much lower than OECD countries which stood at 10.41 tons per capita (IEA 2013). While the level is not that alarming and there may be no urgency in reducing Philippine households' carbon emissions, it is nevertheless relevant to scrutinize factors affecting household emissions in order to project future consumption paths. While reducing aggregate consumption may not be an attractive option (Weber and Matthews 2008), households may exert effort in reducing emissions by conserving and using household energy efficiently.

\subsubsection{Household carbon emissions and income}

Table 1.2 shows the summary statistics of the variables used in the analysis. We observe that there is an increase in households' average income and expenditure. Households above poverty line increased from $67 \%$ in 2000 to $71 \%$ in 2006 . We also observe an improvement in the access to electricity. In 2000, only $77 \%$ of the households had access to electricity and in 2006 it increased to $80 \%$ while household size decreased from 5.24 average members in 2000 to 4.94 members in 2006. Our sample households are mostly male-headed, most are married with an average age close to 50 years and around $40 \%$ have an elementary level of education. Quite surprising is the huge difference of households situated in urban areas from $59 \%$ in 2000 to $45 \%$ in 2006 . This huge decline is mainly due to the change in the definition of urban 
areas. In 2003, the NSCB issued a resolution about the adoption of the operational definition of urban areas in the Philippines (NSCB 2003). Before this new definition, previous household surveys relied on the 1970 (old) definition of urban areas ${ }^{7}$. The old definition considered the entirety of central districts or municipalities when classifying whether the area was urban or rural while the new definition explores further and considers whether a barangay is urban or rural ${ }^{8}$. With the adoption of the new definition, many areas were reclassified from urban to rural.

Table 1.2. Summary statistics of household characteristics.

\begin{tabular}{|c|c|c|c|c|c|c|c|c|}
\hline \multirow{2}{*}{ Variable } & \multicolumn{4}{|c|}{2000} & \multicolumn{4}{|c|}{2006} \\
\hline & Mean & Stdev & Min & Max & Mean & Stdev & Min & Max \\
\hline HH income (pesos) & $124,401.2$ & $199,564.7$ & 4273 & $8,441,242$ & $136,527.6$ & $201,746.6$ & 5295 & $7,919,100$ \\
\hline HH expenditure (pesos) & $115,127.5$ & $129,713.6$ & 3763 & $6,189,500$ & $132,364.2$ & $136,397.9$ & 3918 & $4,042,767$ \\
\hline Above poverty line & 0.67 & 0.472 & 0 & 1 & 0.71 & 0.454 & 0 & 1 \\
\hline Age & 48.89 & 13.870 & 15 & 99 & 48.44 & 14.029 & 13 & 99 \\
\hline Male & 0.79 & 0.409 & 0 & 1 & 0.82 & 0.384 & 0 & 1 \\
\hline Single & 0.08 & 0.270 & 0 & 1 & 0.04 & 0.191 & 0 & 1 \\
\hline Married & 0.77 & 0.422 & 0 & 1 & 0.80 & 0.397 & 0 & 1 \\
\hline Widow & 0.15 & 0.359 & 0 & 1 & 0.16 & 0.365 & 0 & 1 \\
\hline Household size & 5.24 & 2.249 & 1 & 19 & 4.94 & 2.200 & 1 & 19 \\
\hline No formal educ & 0.04 & 0.205 & 0 & 1 & 0.03 & 0.179 & 0 & 1 \\
\hline Elementary & 0.39 & 0.487 & 0 & 1 & 0.42 & 0.494 & 0 & 1 \\
\hline High school & 0.30 & 0.460 & 0 & 1 & 0.33 & 0.471 & 0 & 1 \\
\hline At least college & 0.22 & 0.412 & 0 & 1 & 0.21 & 0.411 & 0 & 1 \\
\hline Urban & 0.59 & 0.491 & 0 & 1 & 0.45 & 0.497 & 0 & 1 \\
\hline Access to electricity & 0.77 & 0.423 & 0 & 1 & 0.80 & 0.400 & 0 & 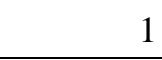 \\
\hline
\end{tabular}

Note: The sample size in 2000 was 37,766 households while in 2006 it was 38,483 households.

One of our main objectives is to investigate how carbon emissions are influenced as households become more affluent. We use expenditure and income data to capture affluence. Results are presented in Table 1.3. The elasticity between expenditure and carbon emissions is captured in the first regression. Results show that there is a significant positive relationship between carbon emissions and expenditure. The elasticity is slightly larger than 1 implying that a change in emissions is proportional to a change in income. In the second regression, the squared term of expenditure is included. We observe a significant nonlinear effect of

\footnotetext{
${ }^{7}$ In the 1970 definition, to be classified as urban (1) cities and municipalities should have a population density of 1000 persons per $\mathrm{km}^{2}$, or (2) central districts should have a population density of at least 500 persons per $\mathrm{km}^{2}$ or (3) regardless of population size, central districts should have street patterns and at least 6 establishments. In the new definition, a barangay is considered as urban (1) if it has a population size of 5000 or more, or (2) if it has at least one establishment with a minimum of 100 employees, or (3) if it has 5 or more establishments with a minimum of 10 employees and 5 or more facilities within $2 \mathrm{~km}$ radius from the barangay hall (NSCB 2003).

${ }^{8}$ Municipalities or central districts are composed of several barangays. Barangay is the smallest administrative and political division in the Philippines.
} 
expenditure on carbon emissions. However, the expenditure variable is endogenous by construction because carbon emissions were estimated based on expenditure. To deal with this problem, we replace the expenditure variable with income. Results show that income has a significant positive effect on emissions but the magnitude of the income coefficient is lower than that of the expenditure. The elasticity is significantly lower than 1 . This is a manifestation of removing the bias that comes with using the expenditure variable. The positive sign on the income coefficient in regression 3 implies a positive monotonic relationship between emissions and income, such that a $1 \%$ increase in household income is associated with an increase in household emissions by $0.80 \%$.

The income-emission elasticity for the Philippine households is 0.80 . This elasticity is higher in comparison to those in the developed countries. For example, Büchs and Schnepf (2013) reported that the income-emission elasticity for the UK households in 2006-2009 was 0.43 while for the US in 2004 it was 0.70 (Weber and Matthews 2008). For Finnish households, the elasticity in 2006 'was 0.79 (Ala-Mantila et al. 2014). Except for Switzerland which posted an elasticity of 0.94 for 2002-2005 (Girod and De Haan 2010), other studies had a relatively lower elasticity as compared to the Philippines. This gives a different perspective on carbon emission between households in developed and developing countries. An increase in income translates to a higher increase in household emissions in developing countries particularly the Philippines as compared to households in the developed countries. This actually mirrors the global situation where the recent increase in carbon emission is largely driven by the rising volume coming from developing countries and not from developed countries (IEA 2013). However, the differences in magnitude of the elasticities could also be attributed to different specifications since these elasticities come from different papers. It would have been better if we had conducted a comparative study on household emissions and compared elasticities among countries. Currently there is no available literature on that issue but Lenzen et al. (2006) did conduct a comparative study on the household energy requirements in Australia, Brazil, Denmark, India and Japan. Our initial findings are supported by their study where they found that Brazil $(e=1.0)$ and India $(e=0.86)$ posted higher elasticities than Australia $(e=0.78)$, Japan $(e=0.64)$ and Denmark $(e=0.86)$. Controlling for similar explanatory variables, households in developing countries posted a higher energyexpenditure elasticity than households in developed countries. This mirrors our claim on income-emission elasticity, where in most cases Philippine households posted a higher elasticity than households in developed countries. This difference could be attributed to differences in energy efficiency, technological innovation or environmental awareness between households in developed and developing countries. If there is no strategic shift in the consumption patterns, it is likely that households from developing countries, particularly the Philippines, will lead a carbon intensive lifestyle.

In the fourth regression, we included the squared term of income to capture the nonlinear effect of income on emission. The result shows that the squared term is negative and significant, depicting an inverted U-shaped relationship between income and household emissions. Holding other factors constant, $\mathrm{CO}_{2}$ emissions rise together with income and then reach a turning point. After reaching the turning point, further increases in income are 
associated with a decline in household emissions. This nonlinear relationship captures the Environmental Kuznets Curve (EKC) hypothesis but the turning point is way beyond the current income distribution. This indicates that a turning point is possible but it is quite far, or it may take too long before a decline in household emission will be observed. This casts doubt about the relevance of the EKC hypothesis at the household level. The EKC is also heavily contested in the literature. Several studies have concluded that the EKC does not exist (Stern 2004; Lenzen et al. 2006; Yaguchi et al. 2007; Galeotti et al. 2008). However in a bivariate regression analysis between income and emission, EKC exists but a cubic relationship is also evident implying a non-monotonic increase in emissions with income (Golley and Meng 2012). Nevertheless, our results show that EKC is present but the turning point is outside the current household incomes. While this turning point cannot be neglected, it also cannot be taken seriously because it is astoundingly high. Household's maximum income is roughly over 8 million pesos and our turning point is roughly around 24 billion pesos. This implies that household emissions are increasing within the current income range and still a huge amount of room to increase before reaching the turning point.

In the fifth regression (Table 1.3), we replace the income variable with the dummy variable depending on whether households fall below the poverty line. Results show that households above the poverty line are $65.7 \%$ higher in emissions than households below the poverty line. This indicates the consumption path of households above poverty is relatively carbon intensive. This poses a challenge in alleviating people out of poverty without aggravating the level of carbon emissions. In the sixth regression, we replace the income variable with income quintiles. We sorted households based on their income and partition them into five groups. The lowest quintile (control group) represents the poorest $20 \%$ of households while the $5^{\text {th }}$ quintile represents the richest $20 \%$. Results show that moving from the lowest quintile to the next quintile increases household carbon emissions by $44 \%$ while moving from the lowest quintile to the highest income quintile increases household carbon emissions by $165.7 \%$. This specification provides a good fit explaining $84 \%$ of the total variation in household carbon emissions. To further analyze the heterogeneity of household emissions by overcoming the potential correlation between control variables and household income, we divide the analysis into two steps. First, we regress household emissions with only income quintiles as covariates (regression 7) then in the second step, our control variables are regressed on the predicted residuals from the previous regression (regression 8). Results show that the highest quintile is $251.3 \%$ higher in emissions compared to the lowest quintile and moving from lowest quintile to the next higher quintile increases carbon emissions by $72.9 \%$. The quintile estimates in regression 7 is relatively higher than the results in regression 6 since there are no other control variables included in regression 7. This shows that income greatly matters in explaining household carbon emissions. 
Table 1.3. Factors affecting household $\mathrm{CO}_{2}$ emissions with $\log$ of $\mathrm{CO}_{2}$ as a dependent variable.

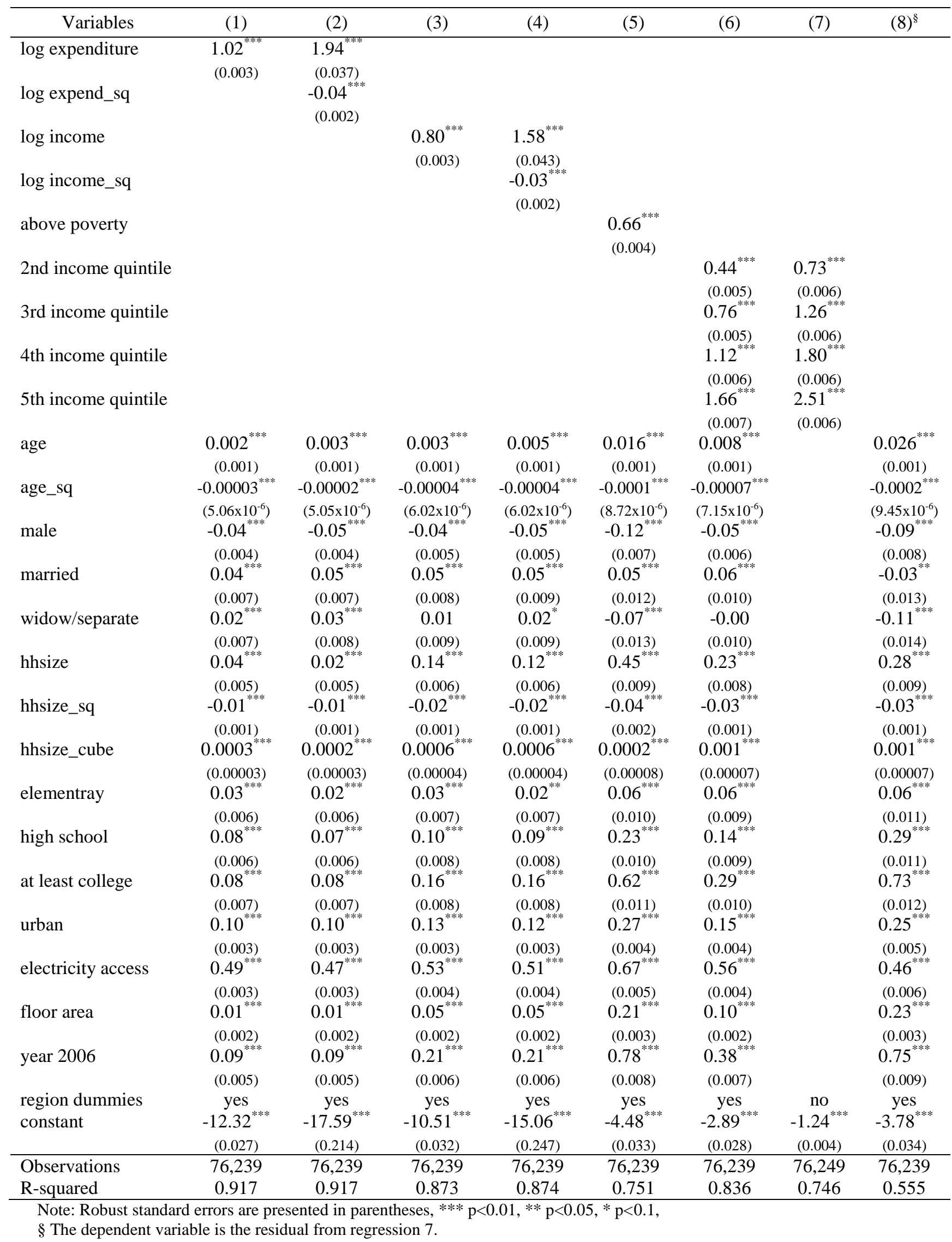




\subsubsection{Carbon emissions and other household characteristics}

Studies on household emissions based on combining input-output and expenditure are often descriptive in nature and only few studies deal with regressing total carbon emissions on socio-demographic characteristics of the households. Hence, our results provide further evidence on the associations between household characteristics and carbon emissions from a developing country's perspective. Although income is the main determinant of household emissions, other household characteristics play an important role in explaining emissions. Information on household age, where they lived, their education, household size and access to electricity matter in explaining the variation in household emissions.

Throughout the different specifications, the control variables behave similarly indicating the robustness of our estimation. Age has a nonlinear effect on carbon emissions depicting an inverse U-shape kind of relationship implying that carbon emissions increase with age until they reach a maximum at a certain age level after which they start to decline. This is due to changes in needs and preferences of the households. Younger households are just starting to raise their family, build houses and accumulate durable goods and this is associated with increasing carbon emissions. As household heads get older, kids grow up and the demand for goods and services also increases, thereby driving carbon emissions even higher. Then later on carbon emissions decline as households reach old age due to changes in preferences and consumption patterns. Older households are more inclined to consume service related goods that are less carbon intensive. This nonlinearity effect of age with emission is consistent with what was reported by Büchs and Schnepf (2013). Also Lenzen et al. (2006) and Golley and Meng (2012) reported a strong influence of age on emissions but they did not include a squared term in their analysis.

In the household survey, we can extract the gender of household head and include them in the regression. Results consistently show that male-headed household posted lower carbon emissions compared to female-headed households. This sounds intriguing but this is plausible because in most household set ups in the Philippines, the husbands tend to focus more on working while housewives tend to handle more of the household expenditures. Although more in-depth research has to be done on this issue, we can speculate that men are more likely to be involved in bigger household expenditures but that as far as expenditures related to food, clothing, and household maintenance women are more assertive. In the literature, limited studies have included gender in their analysis. Büchs and Schnepf (2013) argued that in the UK, while female headed households are less likely to be in the highest emission quartile they have higher home and indirect emissions compared to male headed households.

Most of the available studies did not control for marital status but in this study, we found that marital status significantly influenced household emissions. Being married is associated with higher emissions than single households. Conversely, most studies showed that household size is an important factor in explaining household emissions. We found that household size has a nonlinear effect because there are economies of scale involved or simply put, household members share resources. This sharing of resources among household 
members is also reported in other studies (Lenzen et al. 2006; Druckman and Jackson 2008; Golley and Meng 2012; Büchs and Schnepf 2013). We documented a cubic relationship between household size and carbon emissions and this result is quite robust in all specifications. With smaller household sizes, emissions increase and tend to stabilize at around 4 to 7 household members and then eventually increase further with additional members. The declining marginal emissions in the middle household size captures the sharing of resources among household members but consequently with more added members aggregate household emission will tend to increase.

We also classify households based on the educational attainment of the household head such as (1) no formal education, (2) elementary level, (3) high school level and (4) at least college level. Result shows that better educated household heads have higher carbon emission levels than households headed by someone who has no formal education. Consistent across all regressions, households headed by someone with at least college or university level of education posted higher carbon emissions. This result is in contrast to Baiocchi et al. (2010) but is consistent with Golley and Meng (2012) and Büchs and Schnepf (2013). Lenzen et al. (2006) also reported contrasting effect of education on emissions. They found a negative effect in Australia but a positive effect for Brazil and India. They argue that education is a privilege of the rich, hence, related to higher emissions but since we control for income then this should not be the case. Possible explanations are related to the prestige effect of attaining higher education. Gaining higher education in the Philippines is associated with an elevated social status, and the consumption pattern of such status is carbon intensive. Hence, households headed by someone with a higher education are more likely to consume energy intensive goods. In this case, the argument that better educated households are more aware of the environmental issues is less apparent.

We also found out that households situated in urban areas emit more $\mathrm{CO}_{2}$ than those in rural areas. This is driven by the consumption in energy intensive goods such as fuel, light and transportation. Rural households consume relatively less in fuel, light and transportation than urban households. However, this result is in contrast to Lenzen et al. (2006) and Büchs and Schnepf (2013) where they found that rural locations are associated with higher emissions due to greater car dependency and more isolated dwellings. This is further supported by the evidence presented by Fahmy et al. (2011) and Ala-Mantila (2014). Urban households in developed countries have lower carbon emissions because people live closer to their work place and the proximity of shopping centers and leisure places require less energy for transportation. In addition, urban centers in developed countries have better public transport systems. In contrast as argued before, the set up is quite different for the Philippines. Greater car dependency is observed mostly in the cities and less in rural areas. Public transports such as buses and jeepneys are less available in rural areas than urban areas. Public transport in the Philippines is not efficient resulting to congestion of vehicles in cities and urban areas. Also many households residing in rural areas are out of the energy system. The electrification rate is lower in rural areas than in urban areas. Hence, households in urban areas have higher carbon emissions than rural households. 
In addition, we also included other household characteristics not considered in previous studies. Households who have access to electricity have roughly around 50\% higher carbon emissions than households that have no access to electricity. House size as measured by floor area has a positive significant relationship with total emissions. Golley and Meng (2012) also reported positive a relationship between large dwelling sizes and total emissions. In order to control for geographic variations among households, regional dummies were included in the regressions ${ }^{9}$. Lastly, we also use a time dummy to compare the emissions from 2000 and 2006. The results show that with time we observe an increase in household emission. This shows that holding other factors constant, with time household consumption is shifting to a more carbon intensive lifestyle.

\subsubsection{Household carbon emissions and asset index}

As argued before, the income variable might not be that reliable in capturing households' economic profile. Hence, we run the same regression specifications replacing the income variable with our constructed asset index ${ }^{10}$. The asset index ranges from -4.38 to 7.17, however we rescaled the index so that it ranges on the positive side. Since we took the $\log$ values of household emissions as our dependent variable and also transformed the asset index into $\log$ values, its coefficient can be interpreted as elasticity. Results show that the asset index has a positive significant relationship with household carbon emissions. Holding other factors constant, a percentage increase in asset index increases household carbon emissions by $0.43 \%$ (regression 9, Table 1.4). In regression 10, we added the squared term of the asset index. Results show that the squared term of the asset index has a positive sign indicating that an inverse U-shaped relationship is not evident. This finding shows that as households become more affluent, as represented by its accumulation of assets, emissions tend to increase non-monotonically. This result reflects what was found by Stern (2004), Lenzen et al. (2006), Yaguchi et al. (2007) and Galeotti et al. (2008) showing that carbon emissions did not satisfy the EKC hypothesis but were continually increasing with income.

Though we observe that the EKC hypothesis does not exist when we use the asset index, it is evident when we use the income variable. However, the difference in the squared term between the income variable and the asset index could be attributed to the spread in the distribution. The income variable is unbounded which means households can have different sources of income while the asset index is bounded by the number of assets household could have. In addition, the asset index is also bounded by construction since we use a dummy variable. With this, the asset index may not be able to capture the inverted U-shaped association with emissions as suggested by the income variable.

\footnotetext{
${ }^{9}$ The Philippines is subdivided into 17 regions. We do not report the coefficients, however they are available upon request.

${ }^{10}$ Access to electricity is included in the construction of the asset index. Hence, we remove access to electricity as one of our control variables to avoid correlation with the asset index.
} 
Table 1.4. Determinants of household emissions using asset index as proxy for income.

\begin{tabular}{|c|c|c|c|c|c|}
\hline Variables & (9) & $(10)$ & $(11)$ & (12) & $(13)^{\S}$ \\
\hline \multirow[t]{2}{*}{ log asset } & $0.428^{* * *}$ & $0.357^{* * *}$ & & & \\
\hline & $(0.0037)$ & $(0.0031)$ & & & \\
\hline \multirow[t]{2}{*}{ log asset_sq } & & $0.120^{* * *}$ & & & \\
\hline & & $(0.0016)$ & & & \\
\hline 2nd asset quint & & & 0.361 & 0.535 & \\
\hline \multirow[t]{2}{*}{ 3rd asset quint } & & & $0.663^{* * *}$ & $1.038^{* * * *}$ & \\
\hline & & & $(0.0071)$ & $(0.0079)$ & \\
\hline 4th asset quint & & & $0.929^{* * * *}$ & $1.499^{* * * *}$ & \\
\hline \multirow[t]{2}{*}{ 5th asset quint } & & & $\begin{array}{l}(0.0078) \\
1.228^{* * *}\end{array}$ & $\begin{array}{l}(0.0079) \\
2.076^{* * *}\end{array}$ & \\
\hline & & & $(0.0091)$ & $(0.0079)$ & \\
\hline \multirow[t]{2}{*}{ age } & $0.021^{* * *}$ & $0.019^{* * *}$ & $0.019^{* * *}$ & & $0.015^{* * *}$ \\
\hline & $(0.0010)$ & $(0.0009)$ & $(0.0010)$ & & $(0.0009)$ \\
\hline \multirow[t]{2}{*}{ age_sq } & $-0.000^{* * *}$ & $-0.000^{* * *}$ & $-0.000^{* * *}$ & & $-0.000^{* * *}$ \\
\hline & $(0.0000)$ & $(0.0000)$ & $(0.0000)$ & & $(0.0000)$ \\
\hline \multirow[t]{2}{*}{ male } & $-0.101^{* * *}$ & $-0.079^{* * *}$ & $-0.081^{* * *}$ & & $-0.119^{* * *}$ \\
\hline & $(0.0080)$ & (0.0078) & $(0.0079)$ & & $(0.0073)$ \\
\hline \multirow[t]{2}{*}{ married } & $0.038^{* * *}$ & $0.027^{* *}$ & $0.027^{* *}$ & & $0.036^{* * * *}$ \\
\hline & $(0.0132)$ & $(0.0128)$ & $(0.0130)$ & & $(0.0118)$ \\
\hline \multirow[t]{2}{*}{ widow/separated } & $-0.057^{* * *}$ & $-0.049^{* * *}$ & $-0.053^{* * *}$ & & $-0.060^{* * *}$ \\
\hline & $(0.0139)$ & $(0.0134)$ & $(0.0137)$ & & $(0.0122)$ \\
\hline \multirow[t]{2}{*}{ hhsize } & $0.393^{* * *}$ & $0.377^{* * *}$ & $0.387^{* * *}$ & & $0.151^{* * *}$ \\
\hline & $(0.0107)$ & $(0.0102)$ & $(0.0105)$ & & $(0.0089)$ \\
\hline \multirow[t]{2}{*}{ hhsize_sq } & $-0.044^{* * * *}$ & $-0.041^{* * * *}$ & $-0.043^{* * *}$ & & $-0.022^{* * * *}$ \\
\hline & $(0.0018)$ & $(0.0017)$ & $(0.0018)$ & & $(0.0015)$ \\
\hline \multirow[t]{2}{*}{ hhsize_cube } & $0.002^{* * *}$ & $0.002^{* * * *}$ & $0.002^{* * * *}$ & & $0.001^{* * * *}$ \\
\hline & $(0.0001)$ & $(0.0001)$ & $(0.0001)$ & & $(0.0001)$ \\
\hline \multirow[t]{2}{*}{ elementary } & $0.100^{* * *}$ & $0.116^{* * *}$ & $0.148^{* * *}$ & & $0.159^{* * *}$ \\
\hline & $(0.0122)$ & $(0.0115)$ & $(0.0117)$ & & $(0.0095)$ \\
\hline \multirow[t]{2}{*}{ high school } & $0.317^{* * * *}$ & $0.308^{* * * *}$ & $0.354^{* * * *}$ & & $0.402^{* * * *}$ \\
\hline & $(0.0127)$ & $(0.0121)$ & $(0.0123)$ & & $(0.0102)$ \\
\hline \multirow[t]{2}{*}{ at least college } & $0.700^{* * *}$ & $0.619^{* * *}$ & $0.669^{* * *}$ & & $0.701^{* * * *}$ \\
\hline & $(0.0134)$ & (0.0129) & $(0.0130)$ & & (0.0108) \\
\hline \multirow[t]{2}{*}{ urban } & $0.299^{* * *}$ & $0.259^{* * * *}$ & $0.276^{* * *}$ & & $0.290^{* * * *}$ \\
\hline & $(0.0049)$ & $(0.0047)$ & $(0.0048)$ & & $(0.0048)$ \\
\hline \multirow{2}{*}{ floor area } & $0.221^{* * *}$ & $0.189^{* * * *}$ & $0.205^{* * *}$ & & $0.223^{* * *}$ \\
\hline & $(0.0033)$ & $(0.0033)$ & $(0.0033)$ & & $(0.0031)$ \\
\hline \multirow[t]{2}{*}{ year 2006} & $0.766^{* * * *}$ & $0.672^{* * * *}$ & $0.716^{* * *}$ & & $0.679^{* * * *}$ \\
\hline & $(0.0090)$ & $(0.0089)$ & $(0.0090)$ & & $(0.0082)$ \\
\hline \multirow{3}{*}{$\begin{array}{l}\text { region dummies } \\
\text { constant }\end{array}$} & yes & yes & yes & no & yes \\
\hline & $-4.030^{* * *}$ & $-3.888^{* * *}$ & $-3.992^{* * *}$ & $-1.008^{* * *}$ & $-2.542^{* * * *}$ \\
\hline & $(0.0372)$ & $(0.0361)$ & $(0.0369)$ & $(0.0056)$ & $(0.0327)$ \\
\hline Observations & 76,074 & 76,074 & 76,239 & 76,249 & 76,239 \\
\hline R-squared & 0.692 & 0.716 & 0.707 & 0.525 & 0.430 \\
\hline
\end{tabular}


Similar to the income quintile, we find huge differences in the carbon emissions across quintiles of the asset index. For example in regression 11 (Table 1.4), moving from the lowest quintile to highest quintile increases carbon emissions by $122.8 \%$. This estimate is relatively low compared to the income quintile. The constructed asset index might be potentially correlated with our explanatory variables. To deal with this problem, we proceed with the following two steps. In the first step, we regress household emissions only with the quintiles of the asset index; then, in the second step, the control variables are regressed on the residual from the previous regressions. Results show that coefficients of the asset index behave similarly to income. We observed that moving from the lowest quintile to the next higher quintile increases carbon emissions by $53.5 \%$ and moving from the lowest quintile to the richest quintile increases carbon emissions by more than $200 \%$.

Aside from the asset index, other household characteristics also matters in explaining emissions. The results are consistent with the previous estimation. The nonlinear effect of age and household size still holds. Also being married is associated with higher carbon emissions. Female-headed households are more carbon intensive than male-headed households. This sounds a bit intriguing because men are stereotyped to like cars and having those is carbon intensive, but looking at the asset index shows that only around 6\% only of the households own cars (Appendix Table 4). This means that in other household expenditures women are more assertive. Maybe men are more involved in big household purchases including properties but for everyday expenditure or household comfort women are more likely to be hands on. Hence, on average female-headed households are likely to have higher household emissions than male-headed households. Highly educated households have higher carbon emissions even when we control for income. This shows that the lifestyles of educated households are carbon intensive and that environmental awareness does not come with higher education. This could also be due to the prestige effect of having a higher education in which the effect translates into a carbon intensive lifestyle. Other control variables such as urbanity and larger dwelling places are associated with higher emissions. Lastly, after controlling for income and household characteristics, the dummy variable for year 2006 is consistently higher. This implies that holding other factors constant, with time household consumption are moving towards a carbon intensive lifestyle.

\subsubsection{Household income and consumption patterns}

To evaluate which household items will increase in consumption as households get richer, we use the concept of elasticity. We run separate regressions for every consumption category with income as the main determinant while controlling for the remaining household characteristics (Table 1.5). Goods whose expenditure share increases in consumption as income increases are referred to as normal goods while goods that decrease in consumption as income increases are referred to as inferior goods. 
Results show that as income increases, mostly the expenditure on food related items declines except for meat \& dairy and food eaten out. This is because meat and dairy are relatively expensive food items and are highly valued. Also, households tend to dine out more as their income increases. Aside from food, consumption of alcoholic beverages and tobacco declines as income increases. This may indicate that households are becoming more conscious of health issues because they tend to reduce their consumption on these items. This is also reflected with the increase in medical care as income increases. The expenditure share on fuel, light and water also decline as income increases. This is because energy gets cheaper for richer households or it could be that households are opting for a cleaner source of energy as they become richer. This is a good indication of the possibility to reduce emissions related to the energy intensive consumption of households. Take note that the expenditure on fuel \& light are the most carbon intensive household consumption items. However, this is offset by the increasing expenditure share on transportation as households get richer. Transportation is also a carbon intensive goods that will increase in consumption as households' incomes increase. This finding is consistent among both urban and rural households.

Table 1.5. Income elasticity of household consumption category.

\begin{tabular}{|c|c|c|c|c|c|c|}
\hline \multirow{2}{*}{ Consumption category } & \multicolumn{2}{|l|}{ All } & \multicolumn{2}{|c|}{ Urban } & \multicolumn{2}{|c|}{ Rural } \\
\hline & coef & se & coef & se & coef & se \\
\hline Cereals \& rootcrops & $-0.527^{* * *}$ & 0.003 & $-0.555^{* * *}$ & 0.003 & $-0.517^{* * * *}$ & 0.003 \\
\hline Fruits \& vegetables & $-0.224^{* * *}$ & 0.004 & $-0.245^{\text {**** }}$ & 0.005 & $-0.228^{* * * *}$ & 0.005 \\
\hline Meat \& dairy & $0.075^{* * * *}$ & 0.004 & $-0.020^{* * * *}$ & 0.006 & $0.184^{* * *}$ & 0.007 \\
\hline Fish \& marine goods & $-0.292^{* * *}$ & 0.004 & $-0.365^{* * *}$ & 0.005 & $-0.234^{* * *}$ & 0.006 \\
\hline Non-alcoholic beverages & $0.136^{* * *}$ & 0.006 & $0.033^{* * * *}$ & 0.007 & $0.228^{* * * *}$ & 0.010 \\
\hline Other food & $-0.326^{* * *}$ & 0.004 & $-0.403^{* * *}$ & 0.005 & $-0.280^{* * *}$ & 0.005 \\
\hline Food eaten out & $0.133^{* * *}$ & 0.008 & $0.057^{* * * *}$ & 0.010 & $0.151^{* * *}$ & 0.013 \\
\hline Alcoholic beverages & $-0.161^{* * * *}$ & 0.013 & $-0.231^{* * * *}$ & 0.017 & $-0.156^{* * *}$ & 0.018 \\
\hline Tobacco & $-0.278^{* * *}$ & 0.013 & $-0.357^{* * *}$ & 0.017 & $-0.301^{* * *}$ & 0.019 \\
\hline Fuel, light \& water & $-0.178^{* * *}$ & 0.003 & $-0.160^{* * *}$ & 0.004 & $-0.175^{* * *}$ & 0.005 \\
\hline Transportation & $0.314^{* * *}$ & 0.006 & $0.307^{* * * *}$ & 0.008 & $0.300^{* * * *}$ & 0.009 \\
\hline Communication & $0.476^{* * *}$ & 0.011 & $0.520^{* * * *}$ & 0.013 & $0.417^{* * *}$ & 0.017 \\
\hline Household operation & $0.033^{* * *}$ & 0.005 & $0.136^{* * * *}$ & 0.007 & $-0.077^{* * *}$ & 0.007 \\
\hline Personal care & $-0.016^{* * *}$ & 0.004 & $-0.072^{* * *}$ & 0.005 & $0.045^{* * * *}$ & 0.006 \\
\hline Clothing & $0.199^{* * *}$ & 0.006 & $0.158^{* * * *}$ & 0.008 & $0.252^{\text {**** }}$ & 0.009 \\
\hline Education & $0.356^{* * *}$ & 0.011 & $0.403^{* * *}$ & 0.014 & $0.348^{* * * *}$ & 0.016 \\
\hline Recreation & $0.226^{* * *}$ & 0.012 & $0.287^{* * *}$ & 0.015 & $0.194^{* * * *}$ & 0.019 \\
\hline Medical care & $0.264^{* * *}$ & 0.011 & $0.228^{* * * *}$ & 0.014 & $0.315^{* * * *}$ & 0.015 \\
\hline Nondurable goods & $-0.022^{*}$ & 0.012 & 0.001 & 0.016 & $-0.040^{* *}$ & 0.017 \\
\hline Durable goods & $0.130^{* * *}$ & 0.020 & $0.080^{* * *}$ & 0.023 & $0.221^{* * * *}$ & 0.030 \\
\hline House rent & -0.006 & 0.005 & $0.067^{* * * *}$ & 0.006 & $0.055^{* * * *}$ & 0.007 \\
\hline House repair \& maintenance & $0.119^{* * *}$ & 0.024 & $0.058^{*}$ & 0.032 & $0.191^{* * * *}$ & 0.034 \\
\hline Special occasion & $0.229^{* * *}$ & 0.008 & $0.201^{\text {**** }}$ & 0.011 & $0.330^{* * * *}$ & 0.012 \\
\hline Gifts \& contribution & $0.366^{* * *}$ & 0.010 & $0.384^{* * *}$ & 0.013 & $0.370^{* * *}$ & 0.015 \\
\hline Other expenditure & $0.050^{* * *}$ & 0.008 & $0.140^{* * * *}$ & 0.012 & $-0.101^{* * *}$ & 0.011 \\
\hline
\end{tabular}

Note: Dependent variable is the share of total expenditures allocated to the particular consumption category (in $\log )$. Robust standard errors are used, $* * * \mathrm{p}<0.01, * * \mathrm{p}<0.05, * \mathrm{p}<0.1$ 
To capture lifestyle differences across location, we run separate analysis for households located in urban and rural areas. The elasticity coefficient differs across locations but the majority of both analyses show similar results. As income increases, expenditure on cereals and root crops largely decline as compared to the other goods. Rural and urban households have contradicting elasticities with respect to consumption in meat \& dairy. Urban households show a declining consumption of meat and dairy as income increases while rural households are on the opposite. Another contrasting result is observed expenditures on personal care and household operation.

The priority of the households as their incomes increase is on communication, education, transportation, and expenditures related to gift \& contributions. An increase in the share of transportation will have a strong effect on household carbon emissions driving them upward. Expenditures on recreation, medical care, special occasions, clothing and others also increase as households become more affluent. The elasticities of the remaining consumption categories are shown in Table 1.5 with robust standard errors included.

\subsection{Summary and Conclusion}

In this paper, we estimate the carbon emissions embodied in household consumption and investigate their determinants. We estimate household carbon emissions by extracting $\mathrm{CO}_{2}$ emission intensity from the Philippine Input-Output table and match it with household consumption items. The estimation shows that on average households emitted 1.46 tons of $\mathrm{CO}_{2}$ (0.32 tons of $\mathrm{CO}_{2}$ per capita) in 2000 and 1.86 tons of $\mathrm{CO}_{2}$ in 2006 ( 0.44 tons of $\mathrm{CO}_{2}$ per capita). When disaggregating by major consumption categories, results show that emissions from fuel \& light and transportation occupy a larger share in total emissions when compared to the other consumption items. In addition, there is a huge difference in the carbon emissions between households in the poorest quintile and those in the richest quintile. The increase in emissions is largely due to the increase in emissions from the richest quintile and mostly driven by the increase in emissions from fuel, light and transportation.

Strong results from our regression analyses largely confirm previous findings that emissions will rise with income (Weber and Matthews 2008; Baiocchi et al. 2010; Büchs and Schnepf 2013). The income-emission elasticity in the Philippines, though a little bit lower than 1 , is still higher than most the elasticities in developed countries. This shows that an increase in income translates into a higher increase in household emissions in the Philippines as compared to households in the developed countries. This difference could be attributed to the differences in energy efficiency, technological innovation or environmental awareness between households in developed and developing countries. Unless there is a strategic shift in the consumption pattern, it is likely that households in developing countries, particularly the Philippines, will lead a carbon intensive lifestyle. We even find an inverse U-shaped relationship between income and emissions reflecting the arguments presented by the EKC 
hypothesis but the turning point is way beyond the current income distribution. This implies household emissions are increasing at the current income range and that it will take too long before emissions start to decline. While this concept is contested in the literature (Stern 2004), this effect also vanishes when we use the asset index as a proxy for household economic status. Hence, we cannot strongly claim for an inverse U-shaped relationship between emissions and households' economic status but we can argue here that given the prevailing circumstances, there is strong evidence showing that carbon emissions will likely increase as households get more affluent. The evidence presented by the quintile regression further supports this claim. Results show that there is a dramatic increase in emissions when moving from the lowest quintile to the highest income quintile. This disparity in emissions between the lowest and highest quintile captures how carbon intensive the lifestyle of households become as income increases.

The controls used in the regression are quite robust. The associated signs of the household characteristics and carbon emissions remain as expected from almost all specifications. Age and household size consistently showed a nonlinear effect on household carbon emission. This reflects the change in consumption preference as households get older. Economies of scale or the sharing of resources among household members drives the effect of household size on carbon emissions. Married households have higher carbon emissions than single households. Household headed by someone with higher level of education is associated with higher carbon emissions. This may be due to the prestige effect of attaining higher education. Also, having access to electricity and a larger house is associated with higher household carbon emissions. Results on the association of gender on household emission are quite intriguing. Female-headed households have higher carbon emission than male-headed households. Lastly, in contrast to households in developed countries, we find that households in urban areas have higher carbon emissions than households from rural areas and holding other factors constant, household consumption are becoming more carbon intensive with time.

As compared to households in developed countries, the Philippine households' carbon emissions are still relatively low. They are not worrisome and do not pose a serious threat to the climate system. But as household income increases, carbon emissions will increase drastically. As more households are stepping up the economic ladder, it is imminent that households will lead a carbon intensive lifestyle. With the Philippines' population being close to 100 million, where a quarter of it lives below poverty line (ADB 2014), the increase in the emissions will be enormous and will potentially adversely affect our climate system. This, however, does not imply that income should not increase in order to keep emissions from rising. While household consumption is a matter of private choice and imposing restrictions on it is rather absurd, taxing carbon intensive goods such as fuel, light and transportation can be an option if we aim to control household emissions. Several other options are also possible in curbing household carbon emission. These include improving production efficiency, changing consumption patterns to a less carbon intensive and decreasing consumption of energy intensive goods. However, by doing so policy makers should be cautious not to jeopardize the efforts to reduce poverty in the Philippines. 


\section{Essay 2}

Decomposing drivers of rising household carbon emissions in the Philippines 


\title{
Essay 2: Decomposing drivers of rising household carbon emissions in the Philippines
}

\begin{abstract}
This paper investigates the factors driving rising household carbon emissions in the Philippines using the well-known analytical tool - the Kaya identity. We analyze the change in household carbon emissions, decomposed into factors such as income per capita, carbon intensity and energy intensity, using the logarithmic mean Divisia index. Results show that the increase in household carbon emissions is influenced by the change in income per capita and energy intensity but is evenly offset by the decreasing carbon intensity of household consumption. Income per capita and energy intensity has varying effect across the household distribution. Among poor households, the increase in carbon emissions is largely driven by the income effect while among rich households, the increase in carbon emissions is largely driven by the energy intensity effect. This demonstrates that policy makers should focus their effort in taming energy intensity as a viable option in reducing household carbon emissions.
\end{abstract}




\subsection{Introduction}

According to Duro and Padilla (2006) many factors influence the level of emissions including economic and demographic developments, technological change, resource endowments, institutional framework, lifestyle and international trade. Evaluating these factors is of particular interest to researchers and policy makers in projecting future emission paths and mitigating climate change. Among these factors, our focus for this paper is on people's lifestyles as reflected by their consumption behavior. We continue our work from the previous study (Essay 1) and decompose factors that influence rising household carbon emissions in the Philippines.

The Philippines, which is one of the countries that ratified the convention on climate change, is required to report its national greenhouse gas inventory as stipulated by the IPCC guidelines (UNFCCC 2013; IPCC 2013b). Data from the World Bank shows that the total emissions in the Philippines in 2010 increased around 120\% from its level in 1980 (WDI 2014). In 2010, the average emission per capita in the Philippines amounted to 0.82 tons, much lower than OECD countries which stood at 10.41 tons per capita (IEA 2013). On the household side, our estimation from Essay 1 shows that the per capita emissions in the Philippines is 0.32 tons of $\mathrm{CO}_{2}$ in 2000 and increased to 0.44 tons of $\mathrm{CO}_{2}$ per capita in 2006. The per capita emissions increased almost $40 \%$ in a span of just six years. From Essay 1, we know that income is the main determinant of household emissions and other household characteristics matter in explaining emissions. For this essay, we explore other underlying factors such as changes in energy consumption and carbon intensity that could influence rising household emissions in the Philippines. Hence, we explore these factors by decomposing household carbon emissions.

The literature is abundant with decomposition studies analyzing factors influencing carbon emissions (Sun 1999; Lise 2006; Bhattacharyya and Matsumura 2010; Vinuya et al. 2010; US EIA 2013). The IPCC Working Group III used the Kaya identity in analyzing the major factors influencing global carbon emissions (IPCC 2005). In addition, $\mathrm{Xu}$ and Ang (2013) reviewed around 80 papers using an index decomposition analysis applied to $\mathrm{CO}_{2}$ emissions. However, studies focusing on the emission decomposition on the household level are largely unavailable and decomposition studies from developing countries are very limited. Hence, we fill this two-prong gap in the literature by analyzing the factors that influence rising household carbon emissions in the Philippines using the well-known Kaya identity. Based on this identity, the per capita carbon emission can be decomposed into three major driving forces namely: carbon intensity, energy intensity and affluence. This is another novelty in our paper because the Kaya identity is mostly applied in country level analyses. As of writing, we are not aware of any study that uses the Kaya identity in decomposing carbon emissions at the household level. We will show in this paper that it can also be applied on household level by modifying some of the variables used in the equation. In addition, we analyze the change in the major factors influencing household emissions using the logarithmic mean Divisia index. 


\subsection{Kaya factors of household carbon emissions}

To address the objectives of this study, we proceed in two major steps. First, we decompose household emission into Kaya factors and then in the second step, we use the log mean Divisia index to investigate the change in household carbon emissions as influenced by major driving factors.

We use the famous Kaya identity ${ }^{11}$ in decomposing the driving factors affecting household carbon emissions. It states that the total carbon emissions can be expressed as a product of four basic inputs: carbon intensity per energy consumed, energy intensity per unit of GDP, GDP per capita and population. The Kaya identity as an analytical tool has been frequently used to explore the driving forces of global emissions (IPCC 2013c). This expression is similar to the IPAT identity where the environmental impact of emission (I) can be expressed as a product of population (P) affluence (A) and technology (T) (Rosa and Dietz 2012; IPCC 2013c). Thus, total carbon emission can be expressed as:

$$
C O_{2 i}=\frac{C O_{2 i}}{E_{i}} * \frac{E_{i}}{G D P_{i}} * \frac{G D P_{i}}{P_{i}} * P_{i}
$$

where $\frac{\mathrm{CO}_{2 i}}{E_{i}}$ reflects the carbon emission per unit of energy consumed. This can also be called carbon intensity or the carbonization index. $\frac{E_{i}}{G D P_{i}}$ means the energy intensity or is defined as the amount of energy consumed per unit of GDP and $\frac{G D P_{i}}{P_{i}}$ is the income per capita. This identity can also be used to analyze per capita emissions, that is:

$$
\frac{C O_{2 i}}{P_{i}}=\frac{C O_{2 i}}{E_{i}} * \frac{E_{i}}{G D P_{i}} * \frac{G D P_{i}}{P_{i}}
$$

This decomposition analysis can be used to investigate the main driving factors of carbon emissions. Grounded on this concept, we will apply a Kaya decomposition at the household level. The total household carbon emissions can be expressed as follows,

$$
C O_{2 h h}=\frac{C O_{2 h h}}{E_{h h}} * \frac{E_{h h}}{I n c_{h h}} * \frac{I n c_{h h}}{M e m_{h h}} * M e m_{h h}
$$

where $\mathrm{CO}_{2 h h}$ is the total carbon emissions of a given household, $\frac{\mathrm{CO}_{2 h h}}{E_{h h}}$ captures the amount of carbon emitted per household energy consumed. Household energy will be computed from the household expenditure on fuel, light and transportation because these are energy intensive household expenditures ${ }^{12}$. Households consume energy directly from expenditures related to

\footnotetext{
${ }^{11}$ Kaya identity was popularized by a Japanese economist Yoichi Kaya (Kaya 1995).

${ }^{12}$ This presents a challenge for consistent estimation because prices are not homogenous. For example, the cost of electricity or gasoline differs across location. It would have been ideal to have actual consumption in terms of quantity but unfortunately we do not have that data hence we proxy consumption with expenditure.
} 
fuel, light and transportation. From the descriptive statistics in Essay 1, the share of carbon emissions related to fuel, light and transportation occupies almost $70 \%$ of the total household emissions. For this reason, we focus only on this energy intensive consumption. Although other household expenditures including food, clothing, services, etc. also consume energy, such energy consumption is usually embedded in the production making it indirect energy consumption. Hence, we focus only on the influence of direct energy consumption on household emissions in this decomposition approach. $\frac{E_{h h}}{I n c_{h h}}$ reflects the ratio of household energy expenditure to total household income. $\frac{I n c_{h h}}{M e m_{h h}}$ is the household income divided among household members $\left(\mathrm{Mem}_{h h}\right)$ or basically the income per capita. Similarly, the Kaya decomposition can also be used to analyze household carbon emissions per capita, that is,

$$
\frac{C O_{2 h h}}{M e m_{h h}}=\frac{C O_{2 h h}}{E_{h h}} * \frac{E_{h h}}{I n c_{h h}} * \frac{I n c_{h h}}{M e m_{h h}}
$$

Alternatively, we can represent equation (2.4) by

$$
\mathrm{emit}=\mathrm{CO}_{2} e^{*} \mathrm{En} * \mathrm{Inc}
$$

where emit captures emissions per capita, $\mathrm{CO}_{2} e$ is the carbon intensity, En represents the energy intensity or the ratio of expenditure on energy to income and $I n c$ is the income per capita. Due to data limitation, we deviate from the common definition of carbon and energy intensity. Instead of using quantity of energy used we utilize expenditure on energy because the household survey does not have information on the quantity of energy used.

\subsection{Decomposing change in per capita emissions}

To analyze the change in per capita emissions from 2000 to 2006 as influenced by the household Kaya factors, we apply the decomposition method of logarithmic mean Divisia index (LDMI) (Ang 2004). We apply the additive approach to investigate the difference of household carbon emission from 2000 to 2006. Ang et al. (1998) and Ang and Liu (2001) provided a detailed approach in using LDMI approach to decomposition analysis. This method offers perfect decomposition and has no residual term. We follow their method and we can express the change in household carbon emission as,

$$
\Delta E m i t=\mathrm{Emit}^{06}-\mathrm{Emit}^{00}=\Delta \mathrm{CO}_{2} e+\Delta \mathrm{En}+\Delta \mathrm{Inc}
$$

where

$$
\begin{gathered}
\Delta \mathrm{CO}_{2} e=\sum_{i} \frac{E m i t_{i}^{06}-E m i t_{i}^{00}}{\operatorname{lnEmit}_{i}^{06}-\ln E m i t_{i}^{00}} \ln \left(\frac{\mathrm{CO}_{2} e^{06}}{\mathrm{CO}_{2} e^{00}}\right) \\
\Delta E n=\sum_{i} \frac{E m i t_{i}^{06}-E m i t_{i}^{00}}{\operatorname{lnEmit}_{i}^{06}-\ln E m i t_{i}^{00}} \ln \left(\frac{E n^{06}}{E n^{00}}\right)
\end{gathered}
$$




$$
\Delta I n c=\sum_{i} \frac{E m i t_{i}^{06}-E m i t_{i}^{00}}{\operatorname{lnEmit}_{i}^{06}-\ln E m i t_{i}^{00}} \ln \left(\frac{\operatorname{Inc}^{06}}{\operatorname{Inc}^{00}}\right)
$$

The term, $\sum_{i} \frac{E m i t_{i}^{06}-E m i t_{i}^{00}}{\operatorname{lnEmit} t_{i}^{06}-\operatorname{lnEmit} t_{i}^{00}}$, in equation (2.7) is the logarithmic average of the household emissions for year 2000 and 2006. This is used as weights for each of the underlying factors influencing the change in household emission. This logarithmic average is an improvement of the existing decomposition method which uses the arithmetic mean as the weight function (Ang et al. 1998). Earlier weighting mechanism had residual values. By using the logarithmic average, it ensures that the decomposition results do not contain a residual term. For detailed discussion on the methods and proof please refer to the papers of Ang et al. (1998) and Ang and Liu (2001).

The change in per capita emissions (Aemit) can be expressed as a sum of the change in carbon intensity $\left(\mathrm{CCO}_{2} e\right)$, change in energy intensity $(\triangle E n)$ and change in income per capita $(\Delta I n c)$. By expressing the change in emissions as a sum of these three factors, we can investigate which factors largely influence rising household carbon emissions. The change in carbon intensity $\left(\mathrm{CCO}_{2} e\right)$ captures the degree of carbon emissions per household energy consumption. This refers to how carbon intensive the households' energy consumption is. The change in energy intensity ( $\triangle E n)$ or simply the ratio of energy expenditure to total income captures the changes in the share of energy expenditure to total income. For this decomposition, we focus only on the energy intensive consumption of the households. Lastly, we capture the influence of the income effect on the household emissions. Essay 1 shows that income is one of the key determinants that drives the surging increase in household carbon emissions.

\subsection{Results and Discussions}

\subsubsection{Household characteristics and Kaya factors}

Table 2.1 presents the Kaya factors across selected household characteristics. The emissions per capita is measured in tons of $\mathrm{CO}_{2}$, income per capita is measured in Philippine pesos, emission intensity is measured in tons of $\mathrm{CO}_{2}$ /pesos and energy intensity is measured as percentage. On the average, we observed that there is an increase in both emissions per capita and income per capita. From 2000 to 2006, the increase in per capita income is roughly around $25 \%$ while the increase in per capita emissions is almost $40 \%$. Other Kaya factors show that carbon intensity declines marginally while the energy intensity increases from $9 \%$ to $10 \%$. This result relates to the elasticity of consumption items presented in Essay 1. We observed that as income increases, the expenditure share on fuel and light declines while the expenditure share of transportation increases. The different sign of the elasticities between fuel and light and transportation is reflected by the decline in carbon intensity and an increase in energy intensity. 
To provide an overview of the distribution of Kaya factors, we disaggregate them into several selected household characteristics. As presented before, urban households have higher carbon emission per capita in both years than rural households. The emission per capita as well as income per capita of urban households is twice that of rural households. Also, the emission intensity or the ratio of carbon emissions to energy expenditure and the energy intensity or the ratio of energy expenditure to income is slightly higher in urban household than in rural. With respect to age, we observed that per capita income and per capita emissions behave similarly across age brackets. In addition, carbon emission intensity and energy intensity are relatively similar across the age distribution of household head. With respect to education, we find it to be positively related with emissions and income per capita. Although the difference in magnitude is very marginal, we also observed a positive relationship between education and emission intensity and between education and energy intensity.

Table 2.1. Kaya factors and household characteristics.

\begin{tabular}{|c|c|c|c|c|c|c|c|c|}
\hline \multirow[t]{2}{*}{$\begin{array}{c}\text { HH } \\
\text { Characteristics }\end{array}$} & \multicolumn{2}{|c|}{$\begin{array}{l}\text { Emission } \\
\text { per capita }\end{array}$} & \multicolumn{2}{|c|}{$\begin{array}{c}\text { Income } \\
\text { per capita }\end{array}$} & \multicolumn{2}{|c|}{$\begin{array}{l}\text { Emission intensity } \\
\left(\mathrm{CO}_{2} / \text { energy exp }\right)\end{array}$} & \multicolumn{2}{|c|}{$\begin{array}{l}\text { Energy intensity } \\
\text { (energy/income) }\end{array}$} \\
\hline & 2000 & 2006 & 2000 & 2006 & 2000 & 2006 & 2000 & 2006 \\
\hline \multicolumn{9}{|l|}{ Location } \\
\hline Rural & 0.17 & 0.25 & 18370.79 & 25092.93 & 0.00012 & 0.00012 & 0.08 & 0.09 \\
\hline Urban & 0.42 & 0.67 & 40081.97 & 55588.90 & 0.00015 & 0.00014 & 0.09 & 0.10 \\
\hline \multicolumn{9}{|l|}{ Age } \\
\hline less than 30 & 0.30 & 0.43 & 28845.19 & 38118.08 & 0.00014 & 0.00013 & 0.08 & 0.09 \\
\hline 30 to 45 & 0.29 & 0.38 & 27542.76 & 33495.82 & 0.00014 & 0.00013 & 0.09 & 0.10 \\
\hline 45 to 60 & 0.34 & 0.47 & 34358.47 & 42301.97 & 0.00013 & 0.00012 & 0.09 & 0.10 \\
\hline above 60 & 0.34 & 0.49 & 33795.01 & 43360.02 & 0.00013 & 0.00013 & 0.09 & 0.10 \\
\hline \multicolumn{9}{|l|}{ Education } \\
\hline No formal ed & 0.13 & 0.16 & 14978.50 & 17685.38 & 0.00011 & 0.00011 & 0.08 & 0.09 \\
\hline Elementary & 0.19 & 0.24 & 18688.73 & 22791.57 & 0.00013 & 0.00012 & 0.08 & 0.09 \\
\hline High School & 0.30 & 0.42 & 27286.03 & 35107.96 & 0.00014 & 0.00013 & 0.09 & 0.10 \\
\hline College & 0.62 & 0.89 & 62558.96 & 79008.04 & 0.00014 & 0.00013 & 0.09 & 0.10 \\
\hline Avel & 0.32 & 0.44 & 31206.86 & 38776.22 & 0.00014 & 0.00013 & 0.09 & 0.10 \\
\hline
\end{tabular}

Source: Authors computation based on the household expenditure survey and the estimation of household carbon emissions from Essay 1.

\subsubsection{Decomposition of household emissions by Kaya factors}

Figure 2.1 shows the component of Kaya identity for both years disaggregated into different income quintiles. The behavior of emissions per capita (Figure 2.1a) mirrors the behavior of income per capita (Figure 2.1b). This suggests a strong correlation between emissions and income. This is in accordance with our previous findings showing positive association between emissions and income. The level of emissions and income is higher in 
2006 than in 2000 and households in the richest quintile have relatively higher emissions compared to the rest of the households. We observed a dramatic increase in both emissions and income per capita across quintiles.

There are many factors that influence a households' level of carbon emissions. With the Kaya decomposition, we highlight two key factors affecting household emissions: carbon intensity and energy intensity. Carbon and energy intensity showed contrasting patterns with each other across income quintiles. As households get richer, carbon intensity depicted an increasing trend while energy intensity posted a slightly declining trend across income quintile (Figure 2.1c \& 2.1d).

On average, poor households have a lower carbon intensity than rich households. This is because rich households consume more carbon intensive goods per energy expenditure than poor households. Poor households are more likely to be out of the energy system by having no access to electricity or consuming only very little energy. However, aggregate carbon intensity or the ratio of emission per energy expenditure declines with time. The level of carbon intensity in 2006 is lower than what was observed in 2000 and this pattern is consistent across income quintiles. This decline in carbon intensity across household quintiles over time can be explained by negative elasticity on fuel and light implying that as income increases, households are spending less on this consumption item or it could also mean that this expenditure related to fuel and light becomes cheaper as household become more affluent. Both urban and rural households display a negative elasticity on fuel and light. Household consumption related to fuel and light is becoming less carbon intensive as manifested by this decline in household carbon intensity. On the macro perspective, this decline in carbon intensity can also be attributed to the increasing share of renewable energy to the total primary energy consumption in the Philippines (Department of Energy 2013). According to the US Energy Information Administration (2011), a decline in carbon intensity can indicate a shift away from fossil fuels and a shift towards less carbon intensive fuels.

Energy intensity captures the ratio of energy expenditure to income. Across income quintiles, we observed a slightly declining energy intensity. However, the trend is not so obvious because the ratio of energy expenditure to total income does not largely vary across quintiles. Households in the richest quintiles seem to have a lower energy intensity compared to households in the lower quintiles. Across quintiles, the level of energy intensity in 2006 is relatively higher than what was reported in 2000. This implies that household consumption is becoming more energy intensive. This can be explained by evaluating the elasticity of household expenditures related to direct energy consumption. In contrast to the elasticity of fuel and light, we found that the elasticity on the other energy intensive consumption, like transportation, is positive (please refer to Table 1.5 of Essay 1). Both rural and urban households posted positive elasticity on the transportation expenditure. This affirms our findings here showing that the increase in household energy intensity is reflected by increasing share of household expenditure on transportation. 


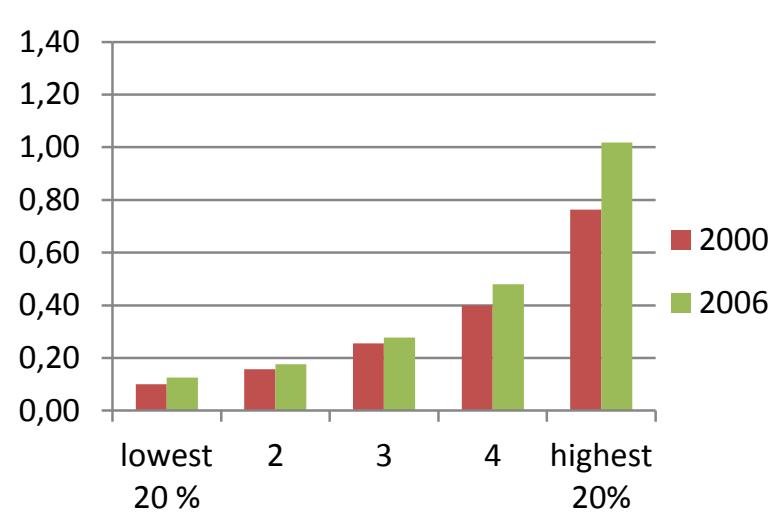

a. $\mathrm{CO}_{2}$ emission per capita

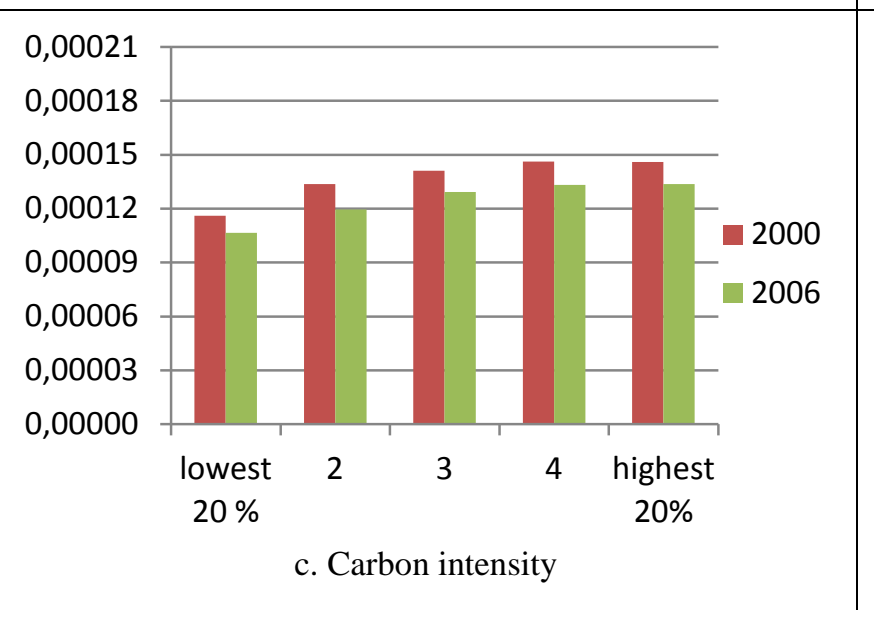

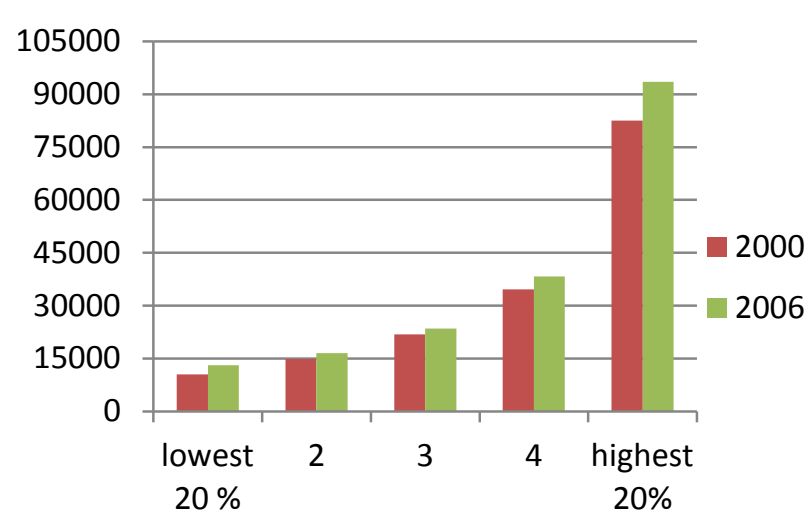

b. Income per capita

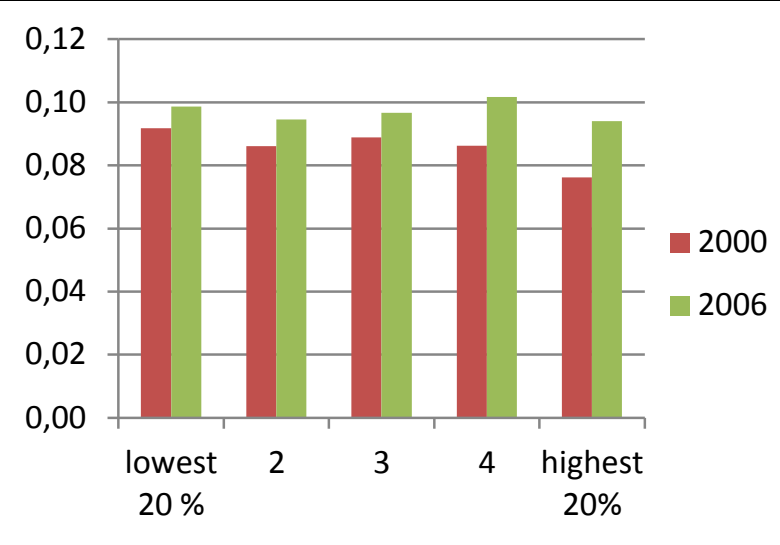

d. Energy intensity

Note: (a) $\mathrm{CO} 2$ emission per capita is measured in tons of $\mathrm{CO} 2$, (b) income per capita is measured in pesos, (c) carbon intensity captures the ratio of $\mathrm{CO} 2$ emission per energy expenditure (tons of $\mathrm{CO} 2$ per pesos) and (d) energy intensity is the ratio of energy expenditure on total income (\%).

Figure 2.1. Decomposition of household emissions by Kaya factors across quintiles.

For policy makers, it is relevant to look at how these drivers affect changes in carbon emission. Figure 2.2 shows the change in household carbon emissions attributed to Kaya factors across income quintiles. We use the method of LMDI to investigate the underlying changes in household carbon emissions decomposed into Kaya factors. The decomposition analysis shows that the richest and poorest quintiles posted the largest change in emissions while the middle quintiles showed relatively modest changes in emissions. The changes in emissions as households become more affluent, as reflected by the different quintiles, depict a U-shaped form. However when accounting for the change in emissions by income quintile, the Kaya factors behave differently. Among poor households (household in the $1^{\text {st }}$ quintile), the change in household emissions is largely due to the income effect while among rich households (households in the $5^{\text {th }}$ quintile), the change in carbon emissions is largely driven by change in energy intensity. This is an interesting observation because the income effect and energy intensity is affecting carbon emissions differently across quintiles. 
The share of the income effect on the change in carbon emissions tends to decline as households move from lower income quintiles to higher quintiles. In contrast, energy intensity increases as households become more affluent. The change in emissions in the richest quintile is mainly driven by the change in energy intensity followed by the change in income. This shows that richer households are consuming more energy intensive goods reflecting the increasing share of income spent on energy related consumption. However, the increase in carbon emissions is offset by a declining rate of carbon intensity. Across income quintiles, we observe that carbon intensity declines. The percentage rate of decrease in carbon intensity is similar across income quintiles. This decline in carbon intensity also mirrors the global scenario where in the majority of the cases in the studies reviewed by $\mathrm{Xu}$ and Ang (2013) showed a decrease in the aggregate carbon intensity in the residential sector. Overall, the change in emissions is largely due to income effect and followed by energy intensity effect.

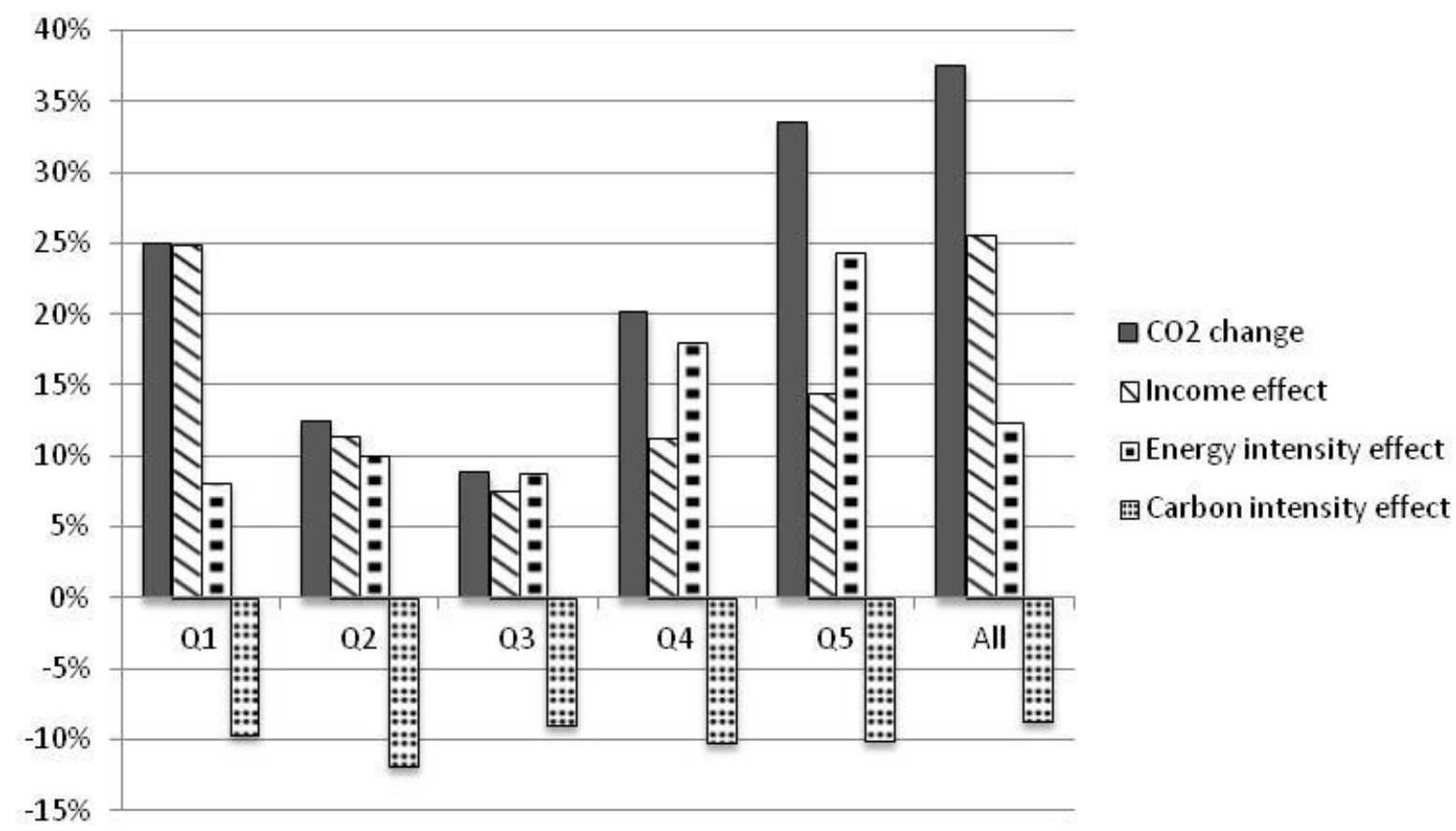

Figure 2.2. Decomposing change in household emissions across income quintiles by Kaya factors.

Figure 2.2 shows that the effect of energy intensity increases as households move from lower income quintiles to richer income quintiles. This result presents a potential roadmap in controlling household emissions. If policy makers are concerned about curbing household emissions, a potential approach is to devise policies related to regulating household energy intensity. This will likely involve an improvement of energy efficiency. This can be done by producing the similar level of output with a lower energy requirement, or by shifting 
production to a less energy intensive one. On the household side, reducing energy intensity means spending less on energy intensive goods yet maintaining the same standard of living. This can be done by consuming, for example, energy efficient household appliances, energy efficient light bulbs, use of more fuel-efficient cars or use of improved public transport. Steckel et al. (2011) also find that emission's growth in China was decelerated by a steady fall in energy intensity in the early 2000. This implies that controlling energy intensity can be a feasible approach in curbing rising household carbon emissions in the Philippines.

\subsection{Conclusion}

We applied the well-known analytical tool, Kaya identity, in investigating major factors driving household carbon emissions in the Philippines. According to Kaya identity, carbon emissions per capita can be expressed as a product of: (i) carbon intensity, (ii) energy intensity and (iii) affluence or income effect. To analyze the underlying changes in per capita emissions, we use logarithmic mean Divisia index. In conclusion, the increase in per capita emissions is largely explained by the change in per capita income and energy intensity but its effect differs across income quintiles. Among poor households, the change in carbon emissions is largely explained by the change in income while among rich households, the change in carbon emissions is mainly driven by the change in energy intensity. Overall, the change carbon emissions is evenly offset by the decrease in carbon intensity of household consumption. While Essay 1 points out the strong correlation between household income and emissions, decomposing the change in emissions shows that this correlation varies across household distribution.

By decomposing household emissions into Kaya factors, we further investigate other relevant factors that could influence rising household carbon emissions. This presents practical approach in if policy makers aim to reduce household emissions. Results suggest that improving energy efficiency is a viable option. This can be done by promoting the use of energy efficient household appliances, energy efficient light bulbs, use of more fuel-efficient cars or use of improved public transport. Theoretically, reducing income per capita can also contribute to reducing emission but that is extremely impractical and very unpopular. Therefore, improving energy efficiency as a way of reducing energy intensity and shifting to cleaner source of energy as a way of further reducing carbon intensity are feasible approaches that can be pursued by policy makers in controlling household carbon emissions 
Essay 3:

Philippine households' carbon footprint inequality: Who walks lightly? 


\title{
Essay 3: Philippine households' carbon footprint inequality: Who walks lightly?
}

\begin{abstract}
This study investigates the household carbon footprint inequality in the Philippines and decomposes it into subgroups applying the standard methods used in analyzing income inequality. Inequality in carbon emissions is one of the most relevant issues for designing climate policies but information on household emission inequality is very limited in the literature. Hence, this study adds to this strand of literature by analyzing the disparity in carbon emissions embodied in households' consumption. We document a high and rising carbon footprint disparity among households. Among poor households, carbon emissions are more unequally distributed than income while among rich households, carbon emissions are more equally distributed than income. The decomposition analysis by income quintiles shows that between-group inequality component is more dominant than the within-group inequality component, however, the opposite finding is observed when decomposing carbon footprint inequality using other household characteristics like urban-rural location, age, household size and education. This demonstrates that income has a strong influence on carbon footprint inequality. Lastly, household carbon footprint inequality is mainly driven by the energy intensive consumption such as fuel, light and transportation. This suggests that policy makers should focus on these carbon intensive consumptions if aiming to control carbon footprint inequality.
\end{abstract}




\subsection{Introduction}

Inequality in carbon emissions is one of the most relevant issues for designing climate change mitigation policies. The emission inequality between developed and developing countries has been one of the biggest challenges hampering the process of forging international agreements towards curbing greenhouse gas emissions (Clarke-Sather et al. 2011; Heil and Wodon 1997). Developed countries fear that limiting their emissions will disrupt their economy while developing and emerging countries argue that their growth should not be limited by any climate mitigation policies because historically their level of carbon emissions have been low (Heil and Wodon 1997; Duro and Padilla 2006). With this polarity of arguments, coming up with global climate mitigation policies has been very challenging. The disparity in emissions across countries or regions is enormously large. Take for example in 1980, according to World Bank (2013), Europe emitted 5.75 tons of $\mathrm{CO}_{2}$ per capita while developing East Asia emitted only 1.27 tons of $\mathrm{CO}_{2}$ per capita. However, this disparity in emission changed in 2009. Much of the increase in emissions was observed from East Asia where the $\mathrm{CO}_{2}$ emissions per capita jumped to 4.59 tons while Europe posted around 7.22 tons of $\mathrm{CO}_{2}$ per capita in 2009 (World Bank 2013).

Though the problem is global, it is also relevant to investigate the situation at the local context. Understanding emission inequality has direct implications towards efforts in reducing carbon emissions at the basic unit of the society. Any mitigation policies aimed at reducing carbon emission have a different effect in a relatively equal society than in a more unequal one. In this vein, this paper examines inequality in the carbon footprint ${ }^{13}$ at the household level. The approach will be twofold. First, we investigate the inequality in the carbon footprint ${ }^{14}$ and its relationship to the income distribution. We analyze the concentration of household emissions through ranking households by income and not by emissions. Second, we will decompose the emission inequality into subgroups to see what drives emission inequality and investigate how it changes over time. Decomposing households' carbon footprint inequality is important because of its policy relevance. It allows us to study whether the change in emission inequality is fueled by a reduction in the emission gap between the rich and poor households or whether the change is due to the homogeneity of lifestyles of households within the same income group. In addition, decomposing emission inequality will also allow us to scrutinize which subgroups largely contribute to the total emission inequality.

Our findings show that the disparity between rich and poor households is larger in the carbon footprint than household income. The inequality in carbon footprint embodied in households' consumption is relatively high and is increasing over time. Decomposing the carbon footprint inequality into household characteristics like location, age, household size and education shows that the within-group inequality component explains a large share of the

\footnotetext{
${ }^{13}$ Carbon footprint refers to the $\mathrm{CO}_{2}$ emissions associated with households' consumption of various goods and services. This definition has been used by Druckman and Jackson (2009) and is in line with the definition suggested by Wiedmann and Minx (2007), Weidema et al. (2008) and Minx et al. (2009).

${ }^{14}$ The term carbon footprint, carbon emissions, and $\mathrm{CO}_{2}$ emissions are used interchangeably in the discussion as they mean the same thing. They capture the embodied $\mathrm{CO}_{2}$ emissions in household consumption.
} 
total emission inequality. In contrast, decomposing emission inequality by income quintiles shows that the between-group inequality component explains a large share of the total emission inequality. This implies that household income exerts a strong influence on the disparity of households' carbon footprint. In addition, we decompose the Gini index by emission source and find that the combined emissions from fuel, light and transportation account for more than 60 percent of the total emission inequality. This suggests that policy makers should focus on these energy intensive consumptions if aiming to curb household emissions inequality.

For comparison purposes, we used studies on emission inequality at the international or national level and examine whether results from the household level are echoed in the broader context. Our findings on the carbon footprint inequality decomposition across income quintiles is similar to what was reported by Heil and Wodon (1997), Padilla and Serrano (2006) and Levy et al. (2009). They found that the emission inequality between income groups of countries carries a greater share to the total emission inequality than the withingroup inequality component. It would have been ideal to compare our results with other emission inequality studies focusing at the household level but such study is unavailable in the literature. Results of the carbon footprint inequality decomposition will hopefully provide valuable information for better policy design concerning distributional issues in reducing household carbon emissions in the Philippines.

\subsection{Literature Review}

A handful of studies have analyzed emission inequality at the international level (Heil and Wodon 1997; Hedenus and Azar 2005; Padilla and Serrano 2006; Cantore and Padilla 2010) while others have focused at the regional level (Alcantara and Duro 2004; Padilla and Duro 2013). While such studies have immediate implications for climate change negotiations, it is nevertheless relevant to investigate emission inequality at the household level. Households contribute to the rising carbon emissions by consuming various goods and services. However, information about household emission inequality in developing countries is very limited. As of writing, this is the first paper that attempts to analyze $\mathrm{CO}_{2}$ emission inequality on a household level in a developing country.

The literature has lately paid attention to the distributional characteristics of $\mathrm{CO}_{2}$ emissions. Heil and Wodon (1997) used a Gini index decomposition to analyze the inequality in $\mathrm{CO}_{2}$ emissions between poor and rich countries. They found that inequality in the cumulative stock of greenhouse gases remains high during the period 1960-1990 and that the between-group component of inequality accounted for half of the inequality in per capita emissions. In another paper, Heil and Wodon (2000) used a Gini group decomposition to forecast what the future inequality in per capita emissions would be using business as usual scenario. Their projection shows that inequality in carbon emissions among 135 countries will decline. This decline is even faster than the decline in income inequality. In addition, even 
though the inequality in carbon emissions declines, their projection found that there would be an enormous increase in $\mathrm{CO}_{2}$ emissions in 2100 relative to the year 1992 and much of this increase will come from developing countries. In another study, Grunewald et al. (2014) decomposed inequality in per capita $\mathrm{CO}_{2}$ emissions across 90 countries and found that emission inequality declined to 0.4 in 2008 from 0.6 in 1971 . This decline is mainly explained by the declining shares and declining inequality in emission of primary energy sources such as coal and oil. In addition, the decline in overall emission inequality is due to the declining emissions from manufacturing and construction.

Another approach conducted by Duro and Padilla (2006) is to decompose emission inequality by using the well known Kaya factors. They decomposed international inequalities in per capita $\mathrm{CO}_{2}$ emissions by carbon intensity, energy intensity and income per capita using the Theil index. The results showed that the inequality in $\mathrm{CO}_{2}$ emissions could be attributed mainly to the variation in per capita income levels. Padilla and Duro (2013) applied this method of decomposing emission inequality by Kaya factor for the case of European Union. The results showed that what was found in the global context is echoed in the regional settings, like in the European Union, where the per capita income is the main explanatory factor of total emission inequality.

Recently, a paper by Duro and Teixidó-Figueras (2013) explores the international inequalities in the ecological footprint ${ }^{15}$ and evidence suggests that the inequality component between groups of countries primarily explains the global inequality. In OECD countries, the decline in the inequality of energy intensity is attributable to both between and with-group component inequality. However, the between-group component contributes a larger share to the total inequality (Alcantara and Duro 2004). These demonstrate that if policy makers are concerned on providing equity in emissions one has to pay attention to the variations between groups. However, there are also cases where the total emission inequality is driven by the within-group component. For example in China, Clarke-Sather et al. (2011) investigated whether the provincial-level inequality in $\mathrm{CO}_{2}$ emissions mirrors the international pattern of emission inequality. Their findings showed that global patterns of $\mathrm{CO}_{2}$ emissions were not mirrored at the sub-national scale because within-group inequality or intraregional inequality contributed more to the total inequality than between group inequality. This is quite in contrast to what was reported by Heil and Wodon (1997), Padilla and Serrano (2006) and Levy et al. (2009) who found that between-group inequality greatly accounts for total emission inequality. This is largely because in China the variations of $\mathrm{CO}_{2}$ emissions across regions are lower than the variation within regions.

Most of the studies on emission inequality are on the international or regional aggregate. However, studies on emission inequality at household level remain largely unexplored in the literature, hence this study.

\footnotetext{
${ }^{15}$ The ecological footprint was first introduced by Rees (1992) and developed by Wackernagel and Rees (1996). It measures the use of resources associated with productive and human activities subject to the bio-productive capacity of the Earth to produce those required resources.
} 


\subsection{Methodology}

\subsubsection{Measures of emission inequality}

We apply the methods usually used in measuring income inequality to household carbon footprint inequality. Groot (2010) showed that such standard tools in measuring income can be applied to the issues of carbon emission inequality. Duro (2012) provides a review detailing the distributive sensitivity of inequality indexes used in measuring emission inequality and suggests the appropriateness of using well-recommended measures in order to achieve robust results for emission inequality. For an index to be a measure of inequality, it should satisfy the following four fundamental properties. First, there is the anonymity principle which states that it does not matter who is earning the income, or in this case, who is emitting. Second, scale independence, which means that if a distribution is scaled up or down by the same percentage, then inequality should not change. For this case, only relative income or emissions matter, not the absolute level of income or emission. Third is population homogeneity. It states that the inequality index should not depend on the size of the population. Doubling the population (and their income or emissions) should not cause a change or shift of inequality. Fourth property of inequality is the Pigou-Dalton transfer principle. This states that if we transfer the income or emissions from the richest household to a poorer household without changing the ranking of the households, the resulting new distribution will be more equal.

Let $y=\left(y_{1}, y_{2}, \ldots, y_{n}\right)$ denote an emission distribution (or, more commonly, an income distribution) for a population of $n$ individuals or households with a mean $\mu$. A measure of emission inequality is defined as a function $I(y)$ designed to determine how much inequality there is for a distribution of emissions. Several methods can be used to measure inequality each one having its own advantages and disadvantages. For this study, we focus on measures of dispersion, concentration and entropy. We use the coefficient of variation to capture the dispersion of household emissions, the Gini and Kakwani index as a measure of concentration and the Theil index as a measure of entropy. These measures are commonly applied in analyzing income inequality. We will use these measures to determine the level of inequality in households' carbon footprint in the Philippines for 2000 and 2006.

A simple way to understand the variation in a given data set is to compute its coefficient of variation $(\mathrm{CV})$. The $\mathrm{CV}$ of household carbon footprint can be obtained by dividing the standard deviation of the carbon footprint by its mean.

$$
C V=\frac{\sqrt{\sum_{i=1}^{n}\left(C F_{i}-\overline{C F}\right)^{2} / n}}{\overline{C F}}
$$

where $n$ refers to the total number of households, $C F_{i}$ is the total carbon footprint of the $i$ th household and $\overline{C F}$ is the mean household carbon footprint. The CV aims to describe the dispersion in carbon footprint between households. However, CV is sensitive to distribution 
where the mean is close to zero. In this case CV is not that meaningful and when the mean is zero, the CV cannot be computed.

The Gini index is a popular index of inequality and is widely used. It is defined as the area of the difference between the line of absolute equality and the Lorenz curve. The Gini is also easy to understand because its value ranges from 0 to 1 where a Gini index of 0 means perfect equality while a Gini index of 1 means perfect inequality. We calculate the Gini index of households' carbon footprint as follows:

$$
G(c f)=\left(\frac{2 \sum_{i=1}^{n} i * C F_{i}}{n \sum_{i=1}^{n} C F_{i}}\right)-\left(\frac{n+1}{n}\right)
$$

where $n$ refers to the total number of households and $C F_{i}$ is the total carbon footprint of the $i$ th household.

Next, we compare the concentration of household income to the concentration of the household carbon footprint. This can be done by comparing the Gini index of household income to the pseudo-Gini index for carbon footprint. The pseudo-Gini index of household carbon footprint measures the inequality in household emission by ranking households according to their income and not according to total carbon footprint. This method was also used by several authors (Padilla and Serrano 2006; Cantore and Padilla 2010; Clarke-Sather et al. 2011). With the pseudo-Gini index of carbon emissions, we investigate the disparity in household carbon footprint between the rich and poor households. The difference between the concentration index of carbon footprint or pseudo-Gini index and the Gini index of household income is commonly known as the Kakwani index.

$$
K=p G(c f)-G(h h)
$$

where $K$ represents the Kakwani index, $p G(c f)$ is the pseudo-Gini index of household carbon footprint and $G(h h)$ is the Gini index of household income. The Kakwani index was originally used to measure progressivity in taxation and public expenditure (Kakwani 1977) and was later applied to equity issues in health care expenditures (Kakwani et al. 1997). The Kakwani index captures the extent to which the inequality in carbon footprint between the poor and rich households differs from the inequality in the income distribution. A negative Kakwani implies that carbon footprint are more equally distributed or less concentrated than household income while a positive Kakwani means that household carbon footprint is less equally distributed or more concentrated than household income (Clarke-Sather et al. 2011).

Lastly, to measure entropy we use the Theil index. It is a weighted entropy index and can be completely decomposed into subgroups. This allows researchers to scrutinize the composition of the inequality into subgroups. The Theil index of households' carbon footprint is calculated as follows: 


$$
T(c f)=\sum_{i=1}^{n} h h_{i} \ln \left(\frac{\overline{C F}}{C F_{i}}\right)
$$

where $h h_{i}$ is the share of household $i$ to the total number of households in the sample, $\overline{C F}$ is the mean household carbon footprint and $n$ is the total number of households. The Theil index can be decomposed into within-group and between-group components as follows:

$$
T(c f)=\sum_{g=1}^{G} h h_{g}\left(T(c f)_{g}+\sum_{g=1}^{G} h h_{g} \ln \left(\frac{\overline{C F}}{C F_{g}}\right)\right.
$$

where $h h_{g}$ is the household share to the total number of households belonging to group $g$, $T(c f)_{g}$ denotes the internal Theil index of households' carbon footprints in group $g$ and $C F_{g}$ represents the household carbon footprint in group $g$. The first term in equation 3.5 is the within-group component of inequality and is a weighted sum of the subgroup inequality values. The second term is the between-group component of inequality.

\subsubsection{Gini decomposition by emission source}

The Gini index of households' carbon footprint is decomposable by source. We first need to divide the total household carbon footprint into mutually exclusive and exhaustive emission sources. Households' carbon footprints are then arranged from lowest to highest. Households are ranked according to their level of carbon emissions. The lowest rank goes to the household with the smallest carbon footprints. For this case, we are dealing with a "simple Gini” of households' carbon footprint (Padilla and Serrano 2006). The overall Gini index of households' carbon footprint can be derived from the individual Gini index of emission source, that is:

$$
G(c f)=\sum_{i} S_{i} R_{i} G(c f)_{i}
$$

where $S_{i}=\frac{\mu_{i}}{\mu}$ is the share of emissions from a particular source in the total household carbon footprint and $R_{i}$ is the rank correlation ratio which can be expressed as $R_{i}=\frac{\operatorname{Cov}\left(y_{i}, r\right)}{\operatorname{Cov}\left(y_{i}, r_{i}\right)}$. $\operatorname{Cov}\left(y_{i}\right.$, $r$ ) is the covariance between the amount of emissions from that particular source and the total emission rank and $\operatorname{Cov}\left(y_{i}, r_{i}\right)$ is the covariance between the amount of emissions from that particular source and the source emission rank.

According to Lerman and Yitzhaki (1985) and as cited by Lopez-Feldman (2006), if one uses a particular technique of the Gini decomposition, one can estimate what effect a small change in a particular source will have on the total inequality. This can be done as follows. Equation 3.6 shows that the overall Gini index of household carbon footprint $G(c f)$ is a product of three terms: (i) the share of the mean emission in that particular source to the overall mean of carbon emissions $\left(S_{i}\right)$, (ii) the correlation of the $i$ th emission source with the 
rank of total household carbon emission $\left(R_{i}\right)$, and (iii) the Gini coefficient of the $i$ th emission source $G(c f)_{i}$, which also can be termed as the emission source Gini.

We can compute the marginal effect of a $1 \%$ change in an emission source to the total emission inequality. Take for example an exogenous change in household's emission source $i$ by a factor of say $d$, such that $y_{i}(d)=(1+d) y_{i}$. Then we can capture the change as

$$
\frac{\partial G(c f)}{\partial d}=S_{i}\left[R_{i} G(c f)_{i}-G(c f)\right]
$$

Dividing equation 3.7 by $G(c f)$, yields the following expression:

$$
\frac{\partial G(c f) / \partial d}{G(c f)}=\frac{S_{i} R_{i} G(c f)_{i}}{G(c f)}-S_{i}
$$

This means that the relative effect of a percentage change in source $i$ on the total inequality is equal to the relative contribution of emission source $i$ to the overall carbon emission inequality minus the relative share of emission from source $i$ to the total household carbon emissions.

\subsubsection{Data}

For the Philippines, there is currently no representative dataset that primarily collects information on household carbon emissions. We use the data on household emissions derived from our estimation in Essay 1. To estimate the total carbon footprint embodied in household consumption, we combine (i) the Philippine input-output (IO) table for 2000, (ii) the carbon emission coefficient for different sectors from the Global Trade Analysis Project (GTAP) and (iii) the household expenditure survey from Family Income and Expenditure Survey (FIES). We first extract the carbon intensity of each sector by combining the IO table with the GTAP's emission coefficient. For this case, we employ the methods of input-output analysis in estimating the embodied carbon footprint (Parikh et al. 1997; Lenzen 1998b; Bin and Dowlatabadi 2005; Kok et al. 2006; Baiocchi et al. 2010; Golley and Meng 2012). Secondly, we match the sectors with the items listed in the household expenditure. Based on our estimation, the carbon footprint of Philippine households in 2000 was on average 1.46 tons of $\mathrm{CO}_{2}$ per household ( 0.32 tons of $\mathrm{CO}_{2}$ per capita) and this amount increased to 1.86 tons in 2006 (0.44 tons of $\mathrm{CO}_{2}$ per capita) $)^{16}$.

\footnotetext{
${ }^{16}$ For the details on the estimation of household carbon footprint, please refer to Essay 1.
} 


\subsection{Results and Discussions}

\subsubsection{Emissions and income gap between rich and poor households}

We compare the distribution of the per capita carbon footprint to the distribution of per capita income (Table 3.1). To do this, we sort the households by income, divide them into five quintiles, and then compare the level and share of emissions and income. As mentioned earlier in Essay 1, there is a rising carbon emission gap between the rich and poor households while there is a declining income gap between the rich and poor households. The gap is computed by taking the ratio of the emissions or income of the richest quintile over the poorest quintile. It shows how high the average emissions or income of the rich households are as compared to the poor households. In 2000, the emission gap between the rich and poor was 7.7 and then it increased to 8.5 in 2006. This means that the average per capita emissions of the rich households is 8.5 times higher than that of the poor households. In comparison, the income gap between the rich and the poor in 2000 is 7.9 and then declines to 7.2 in 2006. This is an indication that there is an improvement in the income distribution as opposed to the distribution of the household carbon footprint. This reflects what was reported by Albert and Ramos (2010) that income inequality in the Philippines went slightly down from 2000 to 2006.

Table 3 1. Comparison of the per capita emissions and income gap across quintiles.

\begin{tabular}{lcrrr}
\hline & Emission & & Income & \\
& 2000 & 2006 & 2000 & 2006 \\
\hline Poorest & 0.10 & 0.12 & 10478.41 & 13093.24 \\
2nd & 0.16 & 0.18 & 14811.26 & 16496.18 \\
Middle & 0.26 & 0.28 & 21888.98 & 23524.64 \\
4th & 0.40 & 0.48 & 34624.37 & 38351.60 \\
Richest & 0.77 & 1.02 & 82763.81 & 93634.96 \\
\hline Average & 0.32 & 0.44 & 31247.08 & 38810.29 \\
Gap & 7.7 & 8.5 & 7.9 & 7.2 \\
\hline
\end{tabular}

Note: Gap between per capita emissions and income is derived by taking the ratio of the average emission or income of the richest over the poor. Emission is measured in tons of $\mathrm{CO}_{2}$ per capita while income per capita is measured in pesos (Philippines currency).

\subsubsection{Inequality in households' carbon footprint and its relation to income distribution}

To evaluate the inequality in the carbon footprint between poor and rich households, we rank the households based on their income distribution. By doing this, we can analyze the degree of concentration in household emissions ordered in terms of increasing value of 
household income and not according to emissions ${ }^{17}$. The concentration index, commonly known as the pseudo-Gini index, of household carbon footprint captures the inequality in emissions between the rich and poor households. This shows to what degree the rich households emit more than poor households.

Table 3.2 shows the income inequality and the degree of concentration in household carbon footprint. Results show that the two indices are moving in different directions. Income inequality shows a slight decrease while the concentration index or the pseudo $\mathrm{CO}_{2}$ Gini shows a relatively huge increase. This means that in 2000, the households are more unequal in income than in emissions but that in 2006, the opposite is true where households are more unequal in emissions than in income. This increase is driven by the rising emission share of the richest households and the declining emission share of the poorest households. Although there is rising share in both income and emissions among rich households, it is only with emissions that we observed a rather large increase in the share (Table 3.2). In addition, this rising emission inequality could also be affected by the large number of households in the poor quintile who use less energy in their consumption.

A related concept to the concentration of household emissions is the Kakwani index. It captures how emission inequality between poor and rich households differs from the income inequality. This is represented by the difference in the pseudo-Gini index of emissions and the Gini index of household income. We document a declining Gini for income but a rising pseudo-Gini for emissions. The difference in the concentration indexes leads to a different sign of the Kakwani index. In 2000, results show a negative Kakwani index while in 2006, results show a positive Kakwani index. This means that in 2000, the carbon footprint is more equally distributed than the income but in 2006, the carbon footprint tends to be less equally distributed than the income. Evidently, there is a worsening carbon footprint inequality among households in the Philippines, however income inequality tends to improve marginally.

Table 3.2. Income inequality and emission inequality.

\begin{tabular}{ccccc}
\hline Year & Income Gini & Simple $\mathrm{CO}_{2}$ Gini & Pseudo $\mathrm{CO}_{2}$ Gini & Kakwani \\
\hline 2000 & 0.478 & 0.498 & 0.455 & -0.023 \\
2006 & 0.474 & 0.516 & 0.475 & 0.001 \\
\hline
\end{tabular}

Note: The simple Gini is computed by ranking the households based on emissions and not on income but the pseudo Gini of $\mathrm{CO}_{2}$ is computed by ranking the households based on per capita income.

We disaggregate both the income and emission distribution into quintiles to observe in more detail the differences between income and emission inequality (Table 3.3). The lowest quintile represents the poorest $20 \%$ of households and the highest quintile represents the

\footnotetext{
${ }^{17}$ If we rank households based on emission and compute the degree of inequality, we are getting the "simple" Gini index of household emission and not the concentration. For comparison, we also compute the "simple" Gini index of emission and present the results in the next section.
} 
richest $20 \%$. Within the same quintile, we rank the households based on their income and derive the income inequality as well as the concentration index of household emissions. By grouping households into quintiles, we narrow down the huge differences in income and make the households more homogenous in their lifestyles. We observe that both the income or emission inequality in the $2^{\text {nd }}$, middle and $4^{\text {th }}$ quintile is relatively low compared to the poorest and richest quintile. This shows that households in the middle-income quintile have more homogeneous lifestyles than the households in the extreme quintiles. In both years, the richest quintile posted a negative Kakwani index, implying that the distribution in income is more unequal than the distribution in emissions. In contrast, the poorest quintile posted a positive Kakwani index implying that the distribution in emissions is more unequal than the distribution in income.

Among poor households, the inequality in the carbon footprint is larger than the income inequality while among rich households, the inequality in the carbon footprint is smaller than the income inequality. This is represented by the crossing of the Lorenz curve of income and the concentration curve of household carbon footprint ${ }^{18}$ (Figure 3.1). This observation is plausible because among rich households, there is somewhat a threshold in the consumption level while income will be unbounded because rich households have more varied sources of income. Hence, emission inequality, which is derived from consumption, is lower than the income inequality among rich households. In contrast, poor households have very limited sources of income yet their consumption is high and more variable relative to their income. This makes emission inequality higher than the income inequality among poor households.

Table 3.3. Concentration indexes and Kakwani index by income quintile.

\begin{tabular}{lcccccc}
\hline & \multicolumn{2}{c}{ Income Gini } & \multicolumn{2}{c}{ Pseudo $\mathrm{CO}_{2}$ Gini } & \multicolumn{2}{c}{ Kakwani index } \\
& 2000 & 2006 & 2000 & 2006 & 2000 & 2006 \\
\hline Poorest & 0.1647 & 0.1467 & 0.1742 & 0.1669 & 0.0094 & 0.0202 \\
2nd & 0.0699 & 0.0701 & 0.0941 & 0.0867 & 0.0242 & 0.0166 \\
Middle & 0.0710 & 0.0720 & 0.0863 & 0.0885 & 0.0153 & 0.0165 \\
4th & 0.0917 & 0.0918 & 0.0815 & 0.0975 & -0.0102 & 0.0057 \\
Richest & 0.2847 & 0.2853 & 0.2120 & 0.2300 & -0.0727 & -0.0553 \\
\hline Overall & 0.478 & 0.474 & 0.455 & 0.475 & -0.023 & 0.001 \\
\hline
\end{tabular}

\footnotetext{
${ }^{18}$ Both the Lorenz and concentration curve is conditional on income.
} 


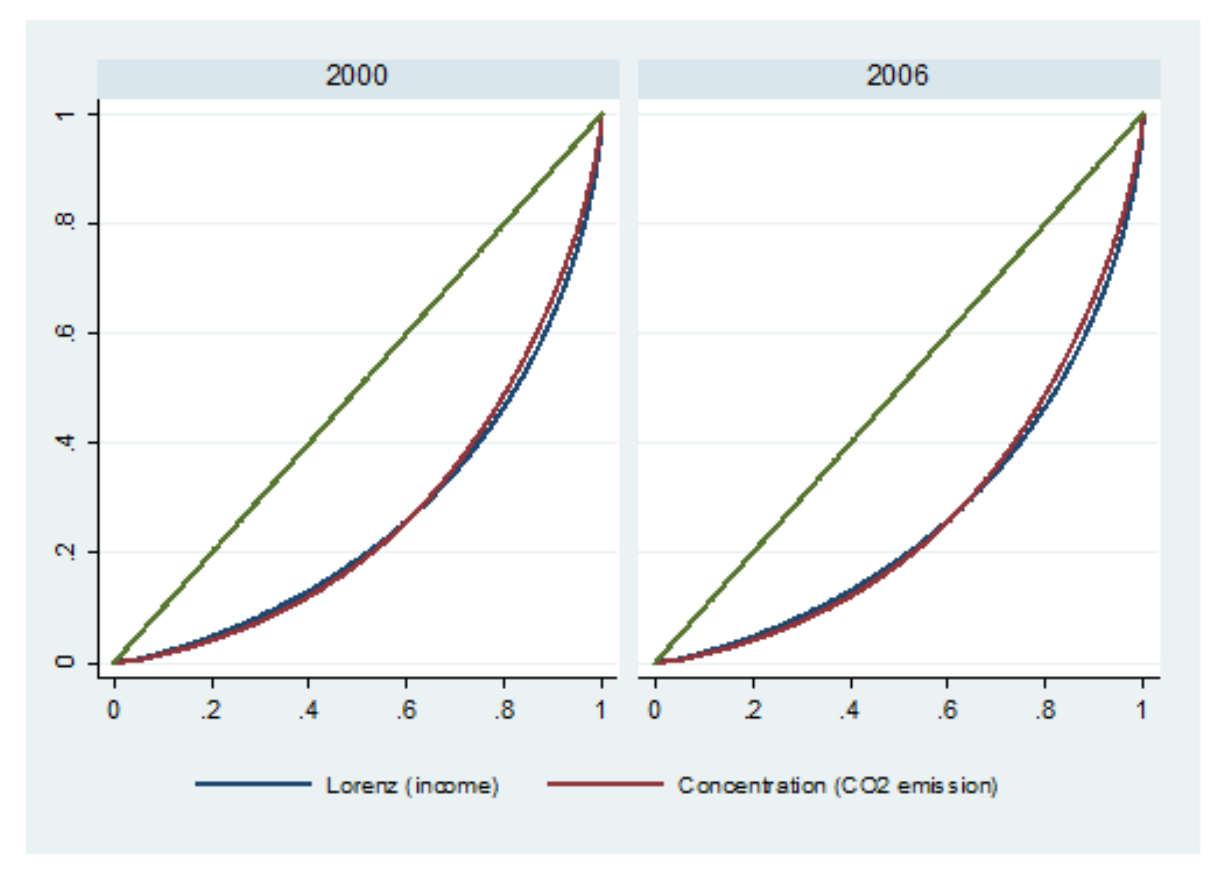

Figure 3.1. Lorenz curve of income and concentration curve of household carbon emissions.

\subsubsection{Simple inequality in household carbon emissions.}

Aside from computing emission inequality ranked by income, we also compute emission inequality based on the ranking of household carbon footprint. Padilla and Serrano (2006) refer to this as the "simple" inequality in carbon emissions. We used the coefficient of variation $(\mathrm{CV})$, Gini index and Theil index to capture the degree of spread, concentration and entropy of households' carbon footprint. We also compute the inequality in income for comparison. Our findings are in rhyme with our previous results revealing that households are more unequally distributed in the carbon footprint than in income (Table 3.4). We also document a worsening inequality in household carbon emissions and a slight improvement income inequality. This observation supports our earlier finding about the concentration of household emission.

Table 3.4 shows that in 2000 households were more unequal in income but were more equal in emissions as measured by the CV. The higher the CV the higher is the degree of disparity among households. By 2006, results reveal that households are more unequal in their carbon footprint than income. With regards to concentration and entropy, the Gini and Theil indexes are consistent in showing that the carbon footprint inequality is higher compared to income in both years. The decline in the Gini index for income is consistent with what was reported by Albert and Ramos (2010) that income inequality in the Philippines went slightly down from 2000 to 2006. This is consistent with Table 3.1 suggesting that there has been a decline in income gap. 
Table 3.4. Household inequality index by emissions, income and expenditure.

\begin{tabular}{lcccccc}
\hline \multirow{2}{*}{ Variables } & \multicolumn{2}{c}{ CV } & \multicolumn{2}{c}{ Gini Index } & \multicolumn{2}{c}{ Theil Index } \\
& 2000 & 2006 & 2000 & 2006 & 2000 & 2006 \\
\hline Emission & 1.284 & 1.253 & 0.498 & 0.516 & 0.468 & 0.493 \\
Income & 1.401 & 1.234 & 0.478 & 0.474 & 0.394 & 0.382 \\
\hline
\end{tabular}

Note: When computing for emission inequality, households are ranked based on their emissions and when computing for income inequality, households are ranked based on income.

\subsubsection{Household characteristics and decomposition of carbon footprint inequality}

We investigate how the carbon footprint inequality is influenced by household characteristics. We decompose inequality into different subgroups such as location, age of household head, household size, education and income quintile. Table 3.5 presents the carbon footprint inequality decomposition by relevant household characteristics.

Results show that the carbon footprint inequality is higher in rural areas than in urban areas. While this may seem contrary to the income inequality where we observe greater income inequality in urban rather than in rural areas (Estudillo 1997), this observation is plausible. It can be explained by evaluating the most carbon intensive consumption items of households such as fuel, light and transportation. Urban households have better access to electricity than rural households. Households in the rural areas are more dispersed and isolated, hence we can expect that the share of households who have no access to electricity is higher in rural areas than in urban areas. This creates a more unequal distribution in emissions. In terms of transportation, car and public transport are used more frequently in urban areas than in rural areas. Consequently, this creates a more unequal carbon footprint distribution in rural areas than in urban areas. Despite having on average a huge carbon footprint gap between urban and rural households, the inequality between these two groups accounts for only a little over $20 \%$. Thus if we eliminate the disparity in household carbon footprint between rural and urban households, the inequality in the carbon footprint will only decline on aggregate by around $20 \%$.

There is a positive relationship between age of the household head and carbon footprint inequality. Both the Gini and Theil indexes reveal that the lowest degree of carbon footprint inequality is observed among young household heads while the highest carbon footprint inequality fell among the oldest household heads.

A U-shaped behavior is observed between emission inequality and household size. Initially with a smaller household size, emission inequality tends to be higher but then with added household members emission inequality tends to decline reaching its lowest peak, after which, emission inequality starts to rise again. This behavior captures the economies of scale or the sharing of resources among household members. With a smaller household size, each member can maximize household resources, hence emission disparity tends to be higher. 
However, with more household members siblings will share resources, thus inequality in emissions tends to decline. But eventually more resources are needed and consumption on the aggregate will rise as household size increases, driving the inequality to increase again.

Classifying households based on the educational attainment shows an inverted Ushaped pattern with emission inequality. Emission inequality is increasing as household heads attain more education and then reaches its peak and eventually, inequality declines again as household heads were able to achieved at least a college level education. The lowest level of emission inequality is observed among households who were able to go to college or university. This implies that highly educated household heads have more homogenous emissions yet their lifestyle is carbon intensive. Take note that college headed households have the largest share of emission at around $42 \%$ as compared to the rest of the households (see Appendix Table 5). This also suggests that gaining higher education is associated with declining emission inequality.

Decomposing carbon footprint inequality by income quintile depicts a U-shaped behavior. The poorest and richest households are more unequally distributed in their carbon footprint than the households in the middle quintile. This result is consistent with our concentration analysis showing that households in the tail end distribution are more heterogeneous in emission as compared to households in the middle-income quintile. This further implies that an increase in income at the lower end of the distribution has an equalizing effect on the carbon footprint while at the upper end of the distribution an increase in income leads to a worsening emission inequality. Greater variations in lifestyle are expected from households in the highest quintile. It is also noticeable that inequality in the highest quintile tends to increase with time while carbon footprint inequality in the lowest quintile decreases with time. This means that on the aggregate level, the increase in carbon footprint inequality is driven by the rising emission inequality in the highest quintile. Looking at the between and within-group inequality component, results shows that total inequality is largely explained by the between-group component.

When we classify households based on location, age, household size and education, results of the inequality decomposition show that the within-group component of inequality explains a greater part of the total carbon footprint inequality than the between-group component. However, this observation is in contrast when inequality is decomposed by income quintiles. Classifying households based on income quintiles shows that the betweengroup component of inequality is larger than the within-group inequality. This shows that household income has a much stronger influence on carbon footprint inequality than the other household characteristics. 
Table 3.5. Inequality decomposition by household characteristics.

\begin{tabular}{lcccc}
\hline \multirow{2}{*}{ Factors } & \multicolumn{2}{c}{ Gini Index } & \multicolumn{2}{c}{ Theil Index } \\
& 2000 & 2006 & 2000 & 2006 \\
\hline Location & & & & \\
Rural & 0.465 & 0.465 & 0.378 & 0.377 \\
Urban & 0.443 & 0.449 & 0.369 & 0.372 \\
Within group (\%) & & & $0.373(79.7 \%)$ & $0.375(76.1 \%)$ \\
Between group (\%) & & & $0.095(20.3 \%)$ & $0.118(23.9 \%)$
\end{tabular}

Age

below 30

0.480

0.479

0.415

0.407

30 to 45

0.475

0.491

0.411

0.428

46 to 60

0.495

0.509

0.465

0.485

above 60

0.536

0.565

0.566

0.625

Within group (\%)

Between group (\%)

$\begin{array}{rr}0.462(98.8 \%) & 0.485(98.4 \%) \\ 0.006(1.2 \%) & 0.008(1.6 \%)\end{array}$

Household size

1 to 3 members

$\begin{array}{ll}0.529 & 0.535 \\ 0.480 & 0.493 \\ 0.482 & 0.516 \\ 0.505 & 0.515\end{array}$

0.541

0.544

4 to 5 members

0.429

0.440

6 to 8 members

0.424

0.480

more than 8

0.467

0.476

Within group (\%)

Between group (\%)

$$
\begin{array}{cc}
0.455(97.3 \%) & 0.481(97.7 \%) \\
0.013(2.7 \%) & 0.012(2.3 \%)
\end{array}
$$

\section{Education}

no formal education

$$
\begin{aligned}
& 0.464 \\
& 0.468 \\
& 0.420 \\
& 0.411
\end{aligned}
$$

0.463

0.382

0.380

elementary

0.475

0.388

0.393

high school

0.439

0.321

0.341

at least college

0.422

0.307

0.324

Within group (\%)

Between group (\%)

$0.348(74.4 \%)$

$0.360(73.1 \%)$

$0.120(25.6 \%)$

$0.133(26.9 \%)$

\section{Income Quintiles}

\begin{tabular}{lcccc} 
poorest 20\% & 0.314 & 0.299 & 0.174 & 0.154 \\
2nd & 0.258 & 0.253 & 0.107 & 0.104 \\
middle & 0.247 & 0.255 & 0.102 & 0.106 \\
4th & 0.229 & 0.243 & 0.089 & 0.099 \\
richest 20\% & 0.306 & 0.322 & 0.158 & 0.173 \\
Within group (\%) & & & $0.127(27.3 \%)$ & $0.127(25.8 \%)$ \\
Between group (\%) & & & $0.340(72.7 \%)$ & $0.366(74.2 \%)$ \\
\hline Overall & $\mathbf{0 . 4 9 8}$ & $\mathbf{0 . 5 1 6}$ & $\mathbf{0 . 4 6 8}$ & $\mathbf{0 . 4 9 3}$ \\
\hline
\end{tabular}


Due to the limited number of available studies related to household emission inequality, we do not have much of a reference point to compare the results of our study. However, we can use information from a macro level emission inequality to check whether our results from the household level mirror the results from the country or regional level. Padilla and Serrano (2006) found that inequality in carbon emissions is mostly explained by the inequality between groups of different income levels, while the inequality within groups of similar incomes is lower. Heil and Wodon (1997) also showed that the between-group and stratification components accounted for half of the inequality in per capita emissions. Levy et al. (2009) reported in their study that there is a stable dominance of emission inequality between five income groups of countries in comparison to inequality within these groups of countries. In addition, Duro and Padilla (2006) also reported in their study about international inequality in per capita $\mathrm{CO}_{2}$ emissions that the between-group component is the biggest contributor to total inequality and is largely explained by the income factor. These findings on the international level reflect what we found on the household level suggesting that the inequality between income groups constitutes a bigger portion to the total carbon footprint inequality than the within-group component.

\subsubsection{Gini index decomposition by emission source}

To determine which consumption sources contributed to the increase in emission inequality, we disaggregate the total household carbon footprint into several consumption categories (Table 3.6). Results show that emissions from fuel and light followed by transportation contributed the largest amount to the total carbon footprint. The contribution of fuel and light to total emissions is $43 \%$ in 2000 and then increases to $46 \%$ in 2006 while transportation's emission share is around $15 \%$ in 2000 and increases to $16 \%$ in 2006 . The combined emission share from fuel, light and transportation accounts to more than $60 \%$ of the total household carbon footprint. However, the emission share from cereals and root crops accounts for only $4.1 \%$ in 2000 and then it even declines to $3.4 \%$ in 2006.

Concerning the inequality share of emission sources, results show that fuel and light contributed more than $50 \%$ of the total carbon footprint inequality followed by transportation, accounting for around $16 \%$ of the Gini index. This shows that aside from occupying a relatively large share in the levels of carbon footprint, consumption from these energy intensive goods also accounts for a large share in the carbon footprint inequality while the rest of the household consumptions occupy a fairly small share. For example, the Gini share of cereals and root crops accounts for only a little over 1\% in both 2000 and 2006.

The decomposition of the Gini index as conducted by Lerman and Yitzhaki (1985) and Stark et al. (1986) allows for the estimation of the marginal effects of emission sources on household carbon footprint inequality. It captures the associated change in inequality as influenced by the change in emission source. A negative marginal effect implies that an increase in emissions from that particular source will have an equalizing effect on the total 
household carbon footprint, while a positive figure will lead to a rise in the carbon footprint inequality. The results show that a $1 \%$ increase in emissions from fuel and light, all else held constant, will lead to a $0.073 \%$ increase in carbon footprint inequality. In contrast, a $1 \%$ increase in emissions from cereals and root crops reduces the total household carbon footprint inequality by a little over $0.020 \%$.

Table 3.6. Decomposition of Gini by emission source.

\begin{tabular}{|c|c|c|c|c|c|c|c|c|c|c|}
\hline \multirow[t]{2}{*}{ Emission Source } & \multicolumn{2}{|c|}{$\begin{array}{c}\text { Emission share } \\
\text { (Sk) }\end{array}$} & \multicolumn{2}{|c|}{$\begin{array}{c}\text { Source Gini } \\
\text { (Gk) }\end{array}$} & \multicolumn{2}{|c|}{$\begin{array}{l}\text { Correlation } \\
\text { (Rk) }\end{array}$} & \multicolumn{2}{|c|}{ Gini Share } & \multicolumn{2}{|c|}{$\%$ Change } \\
\hline & 2000 & 2006 & 2000 & 2006 & 2000 & 2006 & 2000 & 2006 & 2000 & 2006 \\
\hline Cereals \& rootcrops & $4.1 \%$ & $3.4 \%$ & 0.307 & 0.301 & 0.631 & 0.625 & $1.6 \%$ & $1.2 \%$ & -0.025 & -0.021 \\
\hline Fruits \& vegetables & $0.9 \%$ & $0.7 \%$ & 0.398 & 0.373 & 0.702 & 0.677 & $0.5 \%$ & $0.3 \%$ & -0.004 & -0.004 \\
\hline Meat products & $3.5 \%$ & $2.8 \%$ & 0.539 & 0.519 & 0.822 & 0.808 & $3.1 \%$ & $2.3 \%$ & -0.004 & -0.005 \\
\hline Dairy \& egg products & $2.5 \%$ & $2.3 \%$ & 0.541 & 0.571 & 0.730 & 0.722 & $2.0 \%$ & $1.8 \%$ & -0.005 & -0.005 \\
\hline Fish \& marine goods & $5.0 \%$ & $4.2 \%$ & 0.361 & 0.346 & 0.580 & 0.518 & $2.1 \%$ & $1.4 \%$ & -0.029 & -0.027 \\
\hline Coffee, cocoa \& tea & $0.6 \%$ & $0.6 \%$ & 0.407 & 0.380 & 0.255 & 0.301 & $0.1 \%$ & $0.1 \%$ & -0.005 & -0.004 \\
\hline Nonalcoholic drinks & $1.6 \%$ & $1.3 \%$ & 0.598 & 0.585 & 0.678 & 0.719 & $1.3 \%$ & $1.1 \%$ & -0.003 & -0.002 \\
\hline Other food & $3.6 \%$ & $3.2 \%$ & 0.327 & 0.328 & 0.670 & 0.639 & $1.6 \%$ & $1.3 \%$ & -0.020 & -0.019 \\
\hline Alcohol \& tobacco & $1.5 \%$ & $1.2 \%$ & 0.659 & 0.615 & 0.278 & 0.256 & $0.5 \%$ & $0.4 \%$ & -0.009 & -0.009 \\
\hline Water & $4.2 \%$ & $4.3 \%$ & 0.752 & 0.753 & 0.732 & 0.772 & $4.7 \%$ & $4.9 \%$ & 0.004 & 0.006 \\
\hline Fuel \& light & $42.8 \%$ & $45.6 \%$ & 0.611 & 0.624 & 0.955 & 0.960 & $50.1 \%$ & $52.9 \%$ & 0.073 & 0.073 \\
\hline Transportation & $15.2 \%$ & $16.2 \%$ & 0.635 & 0.626 & 0.832 & 0.834 & $16.1 \%$ & $16.4 \%$ & 0.009 & 0.002 \\
\hline Communication & $0.5 \%$ & $0.6 \%$ & 0.901 & 0.767 & 0.871 & 0.876 & $0.7 \%$ & $0.8 \%$ & 0.003 & 0.002 \\
\hline HH operation & $1.4 \%$ & $1.6 \%$ & 0.747 & 0.780 & 0.876 & 0.899 & $1.9 \%$ & $2.1 \%$ & 0.005 & 0.006 \\
\hline Personal care & $2.9 \%$ & $2.6 \%$ & 0.480 & 0.452 & 0.834 & 0.851 & $2.3 \%$ & $2.0 \%$ & -0.006 & -0.007 \\
\hline Clothing \& footwear & $1.8 \%$ & $1.4 \%$ & 0.609 & 0.620 & 0.715 & 0.730 & $1.6 \%$ & $1.2 \%$ & -0.002 & -0.002 \\
\hline Education & $2.8 \%$ & $2.6 \%$ & 0.812 & 0.836 & 0.766 & 0.767 & $3.5 \%$ & $3.2 \%$ & 0.007 & 0.006 \\
\hline Recreation & $0.2 \%$ & $0.2 \%$ & 0.907 & 0.923 & 0.707 & 0.737 & $0.3 \%$ & $0.2 \%$ & 0.001 & 0.001 \\
\hline Medical care & $1.8 \%$ & $2.3 \%$ & 0.874 & 0.865 & 0.674 & 0.692 & $2.2 \%$ & $2.7 \%$ & 0.003 & 0.004 \\
\hline Nondurable goods & $0.2 \%$ & $0.1 \%$ & 0.871 & 0.873 & 0.457 & 0.445 & $0.1 \%$ & $0.1 \%$ & 0.000 & 0.000 \\
\hline Durable goods & $1.2 \%$ & $1.4 \%$ & 0.948 & 0.954 & 0.703 & 0.688 & $1.6 \%$ & $1.8 \%$ & 0.004 & 0.004 \\
\hline Maintenance \& repair & $0.5 \%$ & $0.4 \%$ & 0.957 & 0.966 & 0.534 & 0.495 & $0.6 \%$ & $0.3 \%$ & 0.000 & 0.000 \\
\hline Others & $1.2 \%$ & $1.1 \%$ & 0.872 & 0.869 & 0.744 & 0.771 & $1.6 \%$ & $1.4 \%$ & 0.004 & 0.003 \\
\hline Total & $100.0 \%$ & $100.0 \%$ & 0.498 & 0.516 & & & 0.498 & 0.516 & & \\
\hline
\end{tabular}

An increase in emissions from food related items has an equalizing effect on the household carbon footprint, as manifested by its negative marginal effects. This effect is plausible because an increase in income among poor households will be first utilized to satisfy their basic need for food before spending it on other household needs. However, an increase in emission from fuel, light and transportation, service related consumption and the acquisition of durable goods is associated with worsening effect on carbon footprint inequality. As households get more affluent, they tend to spend their additional income on 
service-oriented goods or other luxury items. This will drive their emissions to a higher level, thereby contributing to a more unequal distribution in households' carbon footprint. This observation is further supported by the Gini index of each particular emission source. Emissions related to food consumption items are more equally distributed than emissions from service-oriented goods.

\subsubsection{Inequality in major consumption categories across income quintiles}

We further decompose the Gini index into six major household consumption items to analyze the internal dynamics of emission inequality. Food summarizes the emissions from food related consumption as well as alcoholic \& non-alcoholic beverages. Energy captures the emissions from fuel and light, while mobility is associated with emissions from household transportation. Operations capture the emissions from communication, nondurable \& durable items, maintenance and other household operations. Clothing includes emissions related to clothing, footwear and personal care while services include education, medical care and recreation. Figure 3.2 shows that the Gini index of household carbon footprint depicts a Ushaped pattern with respect to income. At the lowest quintile, households are more unequal while in the middle quintiles, households' carbon footprint inequality is relatively equal and then at the richer quintile households are more unequal in carbon footprint. This shows that households in the middle income group are more homogenous in their carbon footprint distribution than households in the poorest and richest quintiles. This observation is consistent in both years ${ }^{19}$.

With regards to consumption categories, we would like to highlight the share of inequality related to food consumption and services. Food emission inequality is high among poor households in comparison to other household quintiles while service-related emission inequality is high among rich households in comparison to the other quintiles. These observations are plausible because poor households will prioritize their basic needs such as food while richer households spend more on service-related goods and other luxury goods. Services include expenditures in education, medical care and recreation.

Across quintiles, the disparity in energy intensive consumption dominates the total carbon footprint inequality. Energy related consumption consistently accounts for more than $50 \%$ of the total carbon footprint inequality while mobility accounts for more than $10 \%$ of the total carbon footprint inequality. So, if policy makers are keen on reducing household carbon footprint inequality, then devising policies focusing on consumption related to energy and mobility will provide a more equitable carbon footprint distribution among households.

\footnotetext{
${ }^{19}$ We also did a decomposition of household carbon footprint inequality by quintile and year with results similar to that of Figure 3.2 . Both years depicted a U-shaped kind of relationship between emissions inequality and household income.
} 


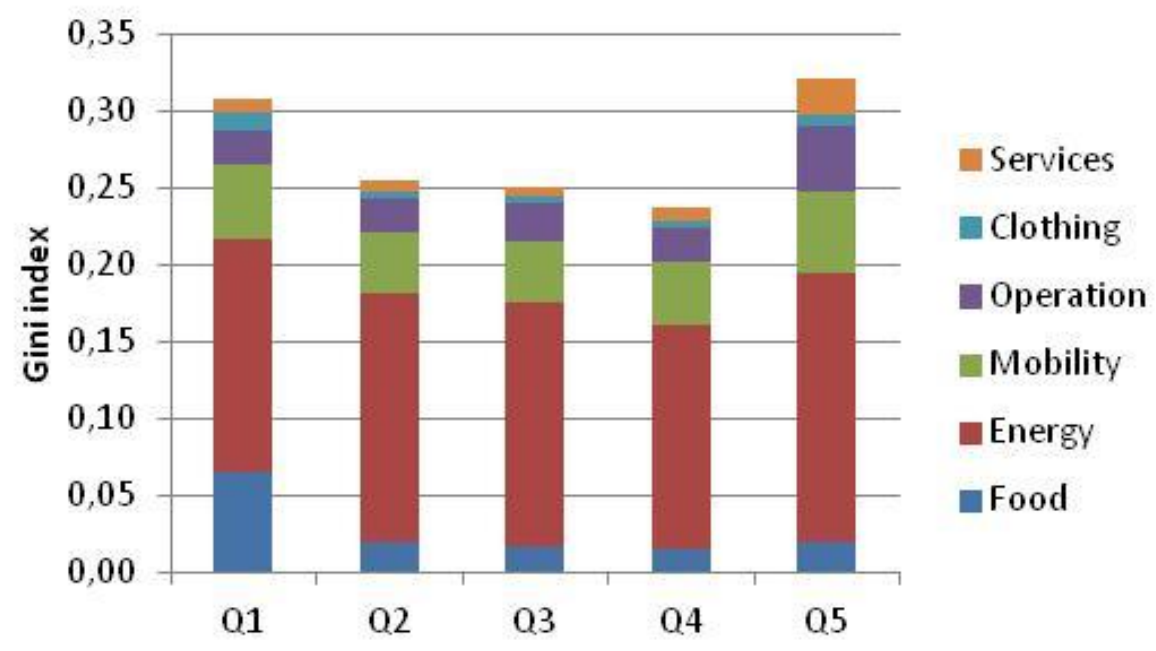

Figure 3.2. Comparison of inequality in major consumption categories across quintiles.

\subsection{Summary and Conclusion}

This study investigates household carbon footprint inequality in the Philippines and decomposes it into subgroups applying the standard methods used in analyzing income inequality. The literature is abundant with evidence on emission inequality at the international level but is very limited on household level. Hence, this study adds to this strand of literature by analyzing the disparity in carbon emissions embodied in households' consumption.

First, we rank the households based on their income and derive the concentration index of carbon footprint. We document a high and rising carbon footprint disparity among households. Poor households are more unequally distributed in carbon emissions than in income while rich households are more unequally distributed in income than in emissions. This is because among poor households, consumption is more variable and higher relative to their income and in contrast among rich households, income is unbounded but their consumption has some threshold.

Second, the inequality decomposition by income quintiles shows that between-group inequality is more dominant than the within-group inequality, however, the opposite finding is observed when decomposing carbon footprint inequality using other household characteristics implying that income has strong influence on carbon footprint inequality.

Lastly, we look at the sources of emission inequality and results reveal that an increase in emission from energy intensive goods such as fuel, light and transportation will worsen total household emission inequality while an increase in emissions from food related consumption will contribute to easing out household inequality. In addition, energy intensive consumption contributes largely to total household carbon footprint inequality. 
Although more urgent and bold steps have to be taken in order to reduce income inequality in the Philippines, policy makers should nevertheless take into account the rising households' carbon footprint inequality. This is important in mitigating climate change and specifically in curtailing overall carbon emissions. Improvement in the standard of living among poor households initially promotes a declining pattern of emission inequality as manifested by the inequality decomposition across income quintiles. However, a balance has to be taken because growth will push emission inequality wider among rich households. This can possibly be altered by introducing carbon tax to certain household consumption items that are carbon or energy intensive but this should potentially target households only in the upper income quintiles so as not push poor households even further down the poverty threshold. The decomposition analysis shows that energy intensive consumption largely influences household carbon footprint inequality. Therefore, if policy makers aim to control inequality in carbon emission, then devising policies focusing on fuel, light and transportation such as fuel taxes will lead to a more equitable carbon footprint distribution among households. In addition, a more potent way of reducing carbon footprint inequality is to provide households with options for green consumption. 


\section{Essay 4}

What motivates developing countries to diversify sources of renewable energy? 


\title{
Essay 4: What motivates developing countries to diversify sources of renewable energy?
}

\begin{abstract}
This paper investigates factors that influence diversification towards nonhydro sources (solar, wind, geothermal, waste and biomass) of renewable energy across 117 developing countries for the period 1980 - 2011. We use new dataset capturing diversification and explore several estimation techniques such as the negative binomial regression, two-part model and Poisson pseudo-maximum likelihood. Results suggest that higher per capita income, implementation of policies promoting renewable energy, advances in technological innovation, access to finance and improvements in human capital promote diversification. We also find robust evidence of a nonlinear effect of income on diversification suggesting a Ushaped kind of relationship. Similarly, high dependence on foreign sources of fuel, increasing world market price for crude oil, and higher population growth will push developing countries to diversify sources of nonhydro renewable energy. In contrast, the local abundance of hydropower and fossil fuel lower the likelihood of diversification. We find no evidence that aid and foreign direct investment supports diversification. In addition, we find robust evidence of diversification in nonhydro sources of renewable energy since the adoption of Kyoto Protocol and lastly, countries with coastal areas are more likely to take advantage of their geographical endowment and move towards diversification of nonhydro sources of renewable energy.
\end{abstract}




\subsection{Introduction}

In this paper, we shift our focus from household emission to renewable energy. The use of renewable energy is seen as an attractive option in mitigating climate change. Besides reducing carbon emissions, renewable energy provides substantial economic benefits such as increased energy access, improved energy security and the utilization of local resources (IPCC 2012; REN21 2013) ${ }^{20}$. However, despite the enormous environmental and socioeconomic benefits associated with renewable energy, its contribution to the total energy supply is still small (IPCC 2012). Though renewable energy is only a minor contributor to total energy supply, its deployment has been rapidly increasing in recent years. It is estimated that renewable energy accounted for $12.9 \%$ of the total primary energy supply in 2008 (IPCC 2012) and in 2011, estimates from REN21 (2013) showed that global demand for renewable energy continued to increase, supplying around $19 \%$ of the global final energy consumption ${ }^{21}$. Despite increasing attention to renewable energy, still there is limited empirical analysis about its determinants, especially in the developing countries. Hence, we fill this gap by investigating what motivates developing countries to diversify sources of nonhydro (solar, wind, geothermal, biomass and waste) renewable energy using a large data set that covers 117 developing countries between 1980 and 2011.

Despite conventional fossil fuels still being the main source of energy, renewable energies are steadily becoming part of the global energy mix. Since the development and innovation of renewable technologies are concentrated in the developed countries, the key challenge for developing countries is to secure the transfer of these climate friendly technologies (Popp et al. 2011; Pfeiffer and Mulder 2013). If renewable energy will be integrated in their energy system, it will likely provide great opportunity for developing countries to leapfrog their development and thereby avoid a lock-in to conventional energy sources (Popp 2011; Watson and Sauter 2011). Although there are already commercially available renewable technologies, still they account for only a limited share of the total amount of energy generated. This is because several market, economic, institutional, technical and socio-cultural barriers hinder developing countries in moving away from fossil fuels (Verbruggen et al. 2010; Dulal et al. 2013). In Africa, shortages of capital, skills and governance hinders its great potential to harness energy from renewable sources (Collier and Venables 2012). Several government policies such as feed-in tariffs, tax credits, tradable certificates, investment incentives and production quotas have been implemented to encourage the adoption of renewable energy (Zhao et al. 2013; IEA 2014). However, much more has to be done to integrate renewable energy in the developing countries' energy mix.

Given the strong relationship between energy demand and economic growth, developing countries are also confronted with the big challenge of growing without further harming the environment. Identifying determinants that promote the diversification of renewable energy in developing countries is therefore strongly warranted. The literature has

\footnotetext{
${ }^{20}$ Renewable Energy Policy Network for the $21{ }^{\text {st }}$ Century (REN21).

${ }^{21}$ Of the $19 \%$ total, $9.3 \%$ come from traditional biomass and $9.7 \%$ come from modern renewable of which $5.2 \%$ come from modern renewable such as solar, wind, geothermal, biomass, $3.7 \%$ from hydropower and $0.8 \%$ from biofuels (REN21 2013).
} 
recently drawn attention to the drivers of renewable energy in developing countries, however there has been quite limited empirical studies done on the issue (see for example Brunnschweiler, 2010; Pfeiffer and Mulder, 2013; Zhao et al., 2013). Our study improves on the recent literature and takes on a different approach by investigating what motivates developing countries to diversify sources of nonhydro renewable energy. According to Brunnschweiler (2010), achieving a diversified and sustainable energy supply for the future is one of the major challenges for today's policymakers.

The main contribution of this paper to the literature is twofold. First, while the usual practice in the literature is to aggregate total energy produced from different sources, we consider a different approach and focus on the issue of diversification. If a country produces renewable energy from only one particular source, we consider it less diversified, while if a country has more than one source of renewable energy then we consider it more diversified. We took two different approaches in capturing diversification. In the first approach, we give equal weights to the different sources by counting the number of nonhydro sources of renewable energy adopted by developing countries. In the second approach, we develop an index of diversification by weighting each source by the share of renewable energy produced from that particular source to the total generation of renewable energy. As of the writing of this paper, this is the first study that attempts to investigate the diversification of nonhydro sources of renewable energy in developing countries. We purposely exclude in the analysis hydropower generation and focus only on modern technologies that harness energy from wind, solar, biomass, geothermal and waste. Second, we particularly focus on developing countries since this is mostly overlooked in the literature concerning renewable energy. There is a need to focus on developing countries about this issue because recently its aggregate level of carbon emissions surpassed that of developed countries. If developing countries will grow now and clean up later, this mechanism is projected to have a catastrophic consequences on our climate system (UNDP 2012). Hence, investigating the possibility of harnessing renewable energy from different sources provides an opportunity for developing countries to evaluate factors that may promote or hinder the adoption of renewable energy and maximize their potential in integrating renewable energy into their energy mix.

Our results are robust to different specifications and estimation approaches. Results suggest that the diversification of nonhydro sources of renewable energy accelerates with higher levels of income, implementation of renewable energy policies, advances in technological innovations, improvements in human capital, access to finance, growing populations and rising oil prices. We also find evidence of the nonlinear effect of income depicting a U-shaped kind of relationship with renewable energy. This suggest that an initial stage of development use of renewable energy is at large but later on declines as the economy develops and shifts to the use of fossil fuels. However, extensive use of fossil fuels is harmful to the environment hence as the society progresses use of clean and renewable energy is desired. This depicts a U-shaped kind of relationship between income and use of renewable energy. In addition, there has been a significant diversification of nonhydro renewable energy since the adoption of Kyoto Protocol in late 1997. This is largely due to the growing number of clean development mechanism (CDM) projects that have been 
implemented in developing countries. In contrast, the abundance of hydropower and the local production of fossil fuels reduce the attractiveness of diversifying nonhydro sources of renewable energy. We also find no evidence showing that aid and foreign direct investment promote renewable energy diversification. Lastly, countries endowed with coastal areas are more likely to diversify nonhydro sources of renewable energy as compared to landlocked countries because of its geographical advantage to potentially tap wind energy and also because of its accessibility providing ease of access to trade and technology transfer.

\subsection{Literature Review}

Despite the rising importance of integrating renewable energy into the country's energy mix, the subject has received little empirical attention. Marques et al. (2010) pointed out that there are increasing numbers of papers on renewable energy but less attention has been given to the discussion about its determinants. A greater portion of the available empirical work is more focused in the developed countries (Menz and Vachon 2006; Carley 2009; Sadorsky 2009a; Marques et al. 2010; Ohler and Fetters 2014) while less attention has been given to the developing countries. This is primarily because the research and development of renewable energy technologies are much more concentrated in the industrialized countries (Popp et al. 2011). Developing countries thus face the challenge of securing transfers of these climate friendly technologies (Pfeiffer and Mulder 2013).

Though the literature on this topic is limited, recent studies on this issue have been rapidly increasing. Menz and Vachon (2006) were the first to use multivariate techniques to assess the effectiveness of different state policies promoting wind power in the United States. They use ordinary least squares (OLS) to examine 39 states between 1998 and 2003 and consider several policy instruments. Results suggest that the development of wind capacity in a given state depends not only on their natural endowment but also on the policies adopted to promote wind power. Key limitations of their study include limited sample size and the possibility of omitted variable problem. Carley (2009) controls for these issues by including a time dimension and estimated fixed effects model with vector decomposition covering 50 states in the US for 9 years (1998 to 2006). Similarly, Marques et al. (2010) used fixed effects vector decomposition to investigate the factors driving renewable energy adoption among 24 European countries for the period 1990-2006 but they did not include policy variables in their analysis. This has been subsequently addressed by Marques and Fuinhas (2012), where they found evidence that policy measures promote a wider use of renewables.

While both Marques et al. (2010) and Marques and Fuinhas (2012) focused only on European countries, recent studies by Pfeiffer and Mulder (2013), Zhao et al. (2013) and Aguirre and Ibikunle (2014) employed a more heterogeneous sample of countries. Aguirre and Ibikunle (2014) include a wider set of countries and use more recent data as compared to Marques and Fuinhas (2012). They include all EU countries, remaining OECD countries and the BRICS. By employing the methods of fixed effects with vector decomposition and panel 
correlated standard errors, their results suggest that some government policies impede investments in renewable energy implying significant failures in design. Zhao et al. (2013) also employed a much broader set of countries in the analysis. They found evidence that policies play a crucial role in promoting renewable energy but the effectiveness of policies diminishes as the number of policies increase. These policies have more pronounced effects in developed and emerging market countries than in developing countries. Despite some failures in policy design, Dulal et al. (2013) argued that the government's role is warranted in the generation of renewable energy, especially in Asia where the increase in population size should be met with dramatic increase in the energy supply. A paper by Johnstone et al. (2010) examines the effect of environmental polices not directly on renewable energy generation but on technological innovation focusing on renewable energy. They use patent data as a proxy for technological innovation on a panel of 25 industrialized countries and found that public policy plays a significant role in determining patent applications and that those policies have varied effects on renewable energy sources. The work of Popp et al. (2011) is related to the study of Johnstone et al. (2010) in the sense that they also use patents in assessing the impact of technological change on investment in renewable energy capacity. They found evidence that technological advances in 26 OECD countries do lead to a greater investment in renewable energy, however the effect is only small.

It is worth noting that the majority of the literature discussed above focuses on developed countries. Only a handful of studies have been found to employ a more heterogeneous sample of developing countries (Brunnschweiler 2010; Pfeiffer and Mulder 2013). In another strand of literature, we have found other studies that focused on relatively few developing countries employing time series analysis (Sadorsky 2009b; Salim and Rafiq 2012) and some studies looking at individual countries (see for example Thiam, 2011; Blenkinsopp et al., 2013; Saidi and Fnaiech, 2014). Brunnschweiler (2010) offers a first attempt to empirically verify the role of finance on renewable energy focusing particularly on non-OECD countries. Results show that the positive effect of financial development on renewable energy is confirmed in a panel data estimation on 119 non-OECD countries for the period 1980-2006.

Most of the studies mentioned above aggregated total energy produced from each renewable source. In this paper, we pay attention to the heterogeneity of sources. We give importance to the variety of nonhydro sources and not just on the total electricity generated. We investigate what motivates developing countries to diversify particularly sources of nonhydro renewable energy. This has not been done before and we attempt to uncover salient information on what motivates developing countries to invest into different sources of nonhydro renewable energy. Our current study is related to Pfeiffer and Mulder (2013) where they focus on the determinants of adopting renewable energy in developing countries. Their findings show that diffusion of renewable energy in developing countries accelerates with implementation of policies promoting renewable energy, higher per capita income and schooling levels and with stable, democratic regimes. In contrast, increasing openness and aid, high fossil fuel production and growing electricity consumption hinders diffusion of renewable energy in developing countries (Pfeiffer and Mulder 2013). The main difference 
between our study and of Pfeiffer and Mulder's (2013) is that we focus on the diversification which gives importance to the different sources of nonhydro renewable energy. Pfeiffer and Mulder (2013) consider only the total electricity generated form nonhydro sources without paying attention to its various sources. For example in Pfeiffer and Mulder's paper, country A which generates 90 kilowatt-hour $(\mathrm{kWh})$ of renewable energy solely from solar energy is equal to country B which also generates $90 \mathrm{kWh}$ in total but from different sources such as solar $(30 \mathrm{kWh})$, wind $(30 \mathrm{kWh})$ and geothermal $(30 \mathrm{kWh})$. For our paper, we say that country $\mathrm{B}$ is more diversified than country A. Diversification of nonhydro sources of renewable energy is crucial in maintaining a sustainable and reliable energy supply. Thus, investigating what motivates developing countries to diversify nonhydro sources of renewable energy is important for devising policies aiming to further promote the diffusion and use of renewable energy in developing countries. Use of renewable energy can be viewed as a feasible approach in reducing global carbon emission without compromising the efforts in reducing poverty in developing countries.

\subsection{Theoretical background and diversification of renewable energy mix}

The concept of diversification is anchored on the theory of portfolio management, which is common in the business and finance literature. Portfolio theory was initially developed by Markowitz (1952) followed by Tobin (1958) and Markowitz (1959) with extensions provided by Lintner (1965). This theory is largely applied in finance but eventually it has also been applied in agricultural economics (Robison and Brake 1979) and more recently on ecology (Figge 2004). A portfolio is simply defined as a combination of items such as assets, securities, crops or other objects of interest, i.e. sources of nonhydro renewable energy. Portfolio theory is used to derive efficient outcomes through various combinations of choices or assets that maximize expected returns for a given level of variance (Barkley et al. 2010). Portfolio theory is a formal representation of the concept of diversification with the aim of selecting an efficient combination of elements that has maximum expected return or lower risk than any individual element. The saying "do not put all your eggs in one basket" is a common expression encouraging diversification.

Markowitz (1952) in his article entitled "Portfolio Selection" published in the Journal of Finance laid out his mathematical arguments favoring portfolio diversification and in order to compare investment options he introduced the concept of efficient frontier. It represents the combinations of asset that has the best possible expected return ("efficient") given certain level of risks. Figure 4.1 is an illustration of the efficient frontier ${ }^{22}$. The efficient frontier attempts to maximize expected return while minimizing risk. Each dot in the graph represents a portfolio and the dots closest to the efficient frontier line are the portfolios that are expected to show the best combination. Similarly, combining several sources of nonhydro renewable energy will yield optimal energy generation.

\footnotetext{
${ }^{22}$ For mathematical formulation of the efficient frontier, please refer to Markowitz (1952).
} 
We can apply the concept of portfolio theory on maximizing returns from diversifying nonhydro sources of renewable energy and minimizing risk from supply distortion. When a country's energy supply relies heavily on one particular energy source it becomes extremely vulnerable to exogenous supply shocks (van Hove 1993). The energy crisis in 1970s had tremendous economic, political and social consequences not just in developed countries but also in developing countries. Since then, policy makers have paid increasing attention to improving energy security. Harnessing energy from local and renewable sources offers the potential to attain energy security especially for countries relying on foreign sources. While renewable energy can contribute to improving energy security, its main disadvantage aside from its large capital cost is the reliability of supply. Most renewable energies rely on the weather as its main source. For example, hydropower needs rain to fill dams and keep the supply of water flowing, wind turbines need wind to turn blades, and solar panels need sunshine and clear skies to generate electricity. These sources are unpredictable and when these sources are unavailable, the supply of energy will be affected. However, diversification of these renewable energy sources can allow for a steady and reliable source of energy supply. For example, dry and sunny weather may not be good for generating hydropower but will be great for generating electricity from solar panels; stormy weather may reduce generation of solar energy but will be good for hydropower, wind energy or tidal energy. Therefore, diversifying sources of renewable energy is essential in minimizing the risk of unreliable energy supply and achieve energy security (Li 2005).

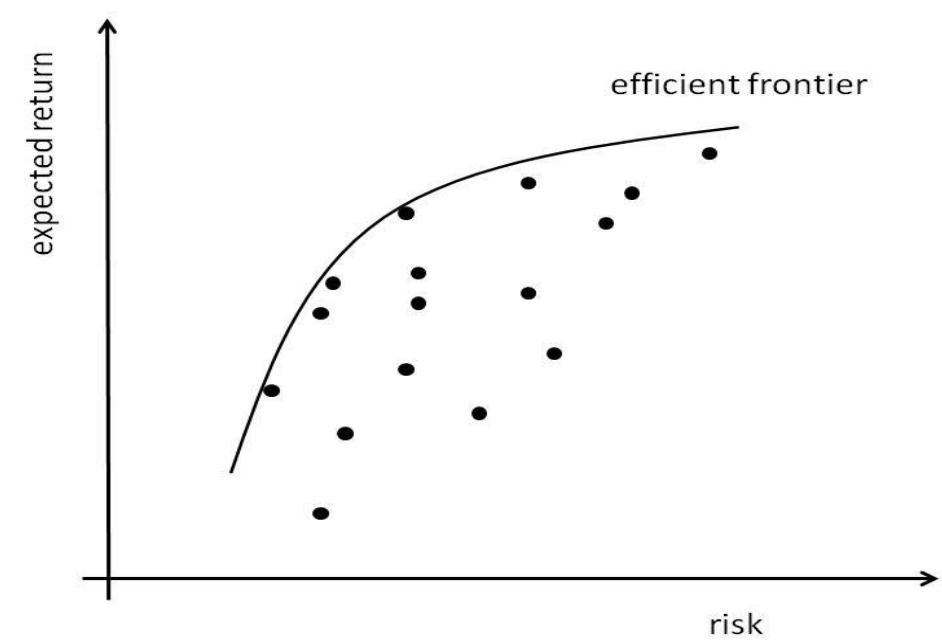

Source: Markowitz (1952)

Figure 4.1. Relationship between expected risk and return.

$\mathrm{Li}$ (2005) argued that diversification and localization of energy sources is essential for future energy system because it promotes sustainable development as well as energy security. $\mathrm{Li}$ (2005) stressed that the idea of diversified energy is good not just for the people but also for the environment. He cited several analogies in other fields pointing out the advantage of diversification. For example, bio-diversity is a good strategy to prevent the spread of pests and diseases, diversified portfolio will guarantee a better investment return and in governance, 
the success of democracy has diversification of ideas at its core. Similarly for renewable energy, diversifying its sources or harnessing energy from a variety of sources is an attractive option for improving energy access, energy security and sustainability. However even if natural endowments such as sunlight, water, wind, geothermal, etc. are available, generation of renewable energy from these varying sources cannot happen in the absence of complementary factors. Collier and Venables (2012) argued that even though Africa is well endowed with potential for hydro and solar power but the scarcity of capital, skills and governance capacity hinders Africa from moving towards renewable energy. Hence, for this current paper, we focus not on the optimization and choosing the best combination of renewable energy sources but rather we focus on investigating factors that hinder or promote diversification of nonhydro sources of renewable energy in developing countries.

\subsection{Data and Methodology}

\subsubsection{Measures of diversification}

Our analysis focuses on the diversification of nonhydro sources of renewable energy in developing countries. The main dependent variable corresponds to the various sources of nonhydro renewable energy adopted by developing countries. We focused only on the newer technologies that harness energy from wind, solar, biomass, waste and geothermal. Brunnschweiler (2010) pointed out that large hydropower cannot viably contribute to sustainable energy production because of the associated negative environmental and social externalities. In addition, hydropower potential is very location specific. For example in SubSaharan Africa, $61 \%$ of the regional hydropower potential is concentrated in just two countries: the Democratic Republic of Congo and Ethiopia (Eberhard et al. 2011). Pfeiffer and Mulder (2013) excluded hydropower in their analysis as Popp et al. (2011) did, who argued that hydropower is a mature technology with less opportunity for technological advances. Following their arguments, we exclude hydropower but we used them as an additional control variable.

Aside from the number of nonhydro sources, we also create an index capturing how diversified are the nonhydro renewable sources adopted by developing countries. We propose capturing the diversity of nonhydro sources by weighting each source with the share of energy generated from its sources to the total renewable energy. To capture the diversity of a country's nonhydro renewable energy mix, we propose using the following method:

$$
\text { dre }=\left\{\begin{array}{cc}
\frac{1}{\sum_{i=1}^{n}\left(\frac{n h r e_{j t}}{N H R E}\right)^{2}} & \text { if NHRE }>0 \\
0 & \text { if NHRE }=0
\end{array}\right.
$$

where dre captures the diversity of a developing country's nonhydro renewable energy mix, $N H R E$ is the net generation of nonhydro-electricity measured in billion kilowatt-hours and the 
$n h r e_{j t}$ is the net energy generation from any of the nonhydro sources such as wind, solar, geothermal, biomass and waste in country $j$ in year $t$. The index takes a value of zero if a country has not invested in any nonhydro sources and takes a positive value if a country has invested in at least one of the nonhydro renewable technologies. The positive value ranges from 1 if the energy generated is coming from only one source and if the country is more diversified then the index is greater than 1 . If each source carries the same weight (the same share) the index converges to the total number of nonhydro sources adopted. For example, compare country A and country B with three different sources of nonhydro renewable energy such as solar, wind and geothermal. Country A decides to generate $80 \%$ energy from solar, $10 \%$ from wind and $10 \%$ from geothermal while country B decides to generate $40 \%$ energy from solar, $30 \%$ from wind and 30\% from geothermal. Based on our formula above, country $\mathrm{B}(d r e=2.94)$ is more diversified than country $\mathrm{A}($ dre $=1.85)$ because country $\mathrm{B}$ has generated more equal share of renewable energy from three different sources while country A relies mostly on energy generated from only one source.

In the literature, there are two commonly known methods of diversification especially used in industrial economics: the Herfindahl index and the entropy measure (Jacquemin and Berry 1979; van Hove 1993). The Herfindahl index (H) usually computes the industry concentration summing the square of market shares, which is:

$$
H=\sum_{i=1}^{n} s_{i}^{2}
$$

where $s_{i}$ is the market share but for our case, $s_{i}$ represents the share of nonhydro energy generated from a particular source to the total nonhydro generation of renewable energy. $\mathrm{H}$ decreases as the number of nonhydro sources of renewable energy increases. When all nonhydro sources have an equal share, $\mathrm{H}$ converges to $1 / \mathrm{n}$ and when there is only one nonhydro source, $\mathrm{H}$ is equals to 1 . To make the index increase with the increasing number of nonhydro sources, the index is redefined as follows:

$$
H=1-\left(\sum_{i=1}^{n} s_{i}^{2}\right)
$$

However with this definition, a country with only 1 nonhydro source will have an index $\mathrm{H}=0$ and by construction countries having no investment in any nonhydro renewable energy will have missing observations. In short, this index will not accommodate zero observation.

The other measure of diversification measures entropy or the inverse measure of concentration. Entropy is formulated as follows:

$$
E=\sum_{i=1}^{n} s_{i} \ln \left(\frac{1}{s_{i}}\right)
$$

where $\mathrm{E}$ is higher when the share of nonhydro sources of renewable energy are more equally spread and $\mathrm{E}=0$ when there is only one source. Herfindahl index will translate to missing values for countries having no investment in any nonhydro sources. Because of this, we refrain from using $\mathrm{H}$ and $\mathrm{E}$ as our measure of diversification. This distorts our observation given that we have a large number of zero observations capturing those developing countries that have not invested in any nonhydro sources. By using the $\mathrm{H}$ and $\mathrm{E}$ index, it focuses only 
on countries that adopted nonhydro sources of renewable energy. For this reason, Herfindahl index and entropy measure might not be applicable in this situation. Thus, to capture the diversification of nonhydro sources while not dropping out countries in our data set that do not have investments in any nonhydro sources, we use the diversity index described in equation 4.1. In addition, we also use the number of nonhydro sources adopted in developing countries as a measure of diversification. For a robustness check, we use the total energy generated from different nonhydro sources. Although the total amount of electricity generated does not directly reflect the diversification as it only sums up all electricity generated from individual nonhydro sources, it provides information on the current capacity of developing countries in harnessing energy from nonhydro sources.

\subsubsection{Data description}

The dataset used in this study covers 117 developing countries from 1980 to 2011. This dataset is compiled using four different sources: Energy Information Administration (EIA), International Energy Agency (IEA), World Development Indicators (WDI) and the BP Statistical Review of World Energy (BP). Our main source of data for the generation of renewable energy comes from the IEA. Data from the IEA on nonhydro electricity generation can be considered comprehensive, however it may have underestimated the amount of energy generated as off-grid generation are not included in the data set (Pfeiffer and Mulder 2013).

To identify factors that may affect the diversification of nonhydro renewable energy, we include several variables highlighted in the literature that influence the adoption of renewable energy. The independent variables included in the analysis are discussed as follows.

(1) The effect of income is widely captured in the literature (Marques et al. 2010; Popp et al. 2011; Zhao et al. 2013; Aguirre and Ibikunle 2014). Higher income will enable countries to invest in varied sources of renewable energy. To capture the nonlinear effect of income on renewable energy, which is often neglected in the previous studies, we include the squared term of income in the analysis.

(2) Energy imports capture the degree of dependence on external sources. We expect that greater dependence on foreign sources of energy will fuel investment on renewable energy to improve energy security (Marques et al. 2010; Popp et al. 2011; Dong 2012; Zhao et al. 2013; Aguirre and Ibikunle 2014).

(3) While most of the available studies did not include population growth, we hypothesize that an increase in population will drive up demand for energy and this will positively contribute to the development of renewable energy as the country tries to address the increasing energy needs of its population.

(4) Adopting renewable technologies can be facilitated by the degree of technological advances in developing countries. We use total patents as a proxy for technological innovation in the developing countries. Popp et al. (2011) used patent data to assess the role of technological innovation in advancing investment on renewable 
technologies and found that technological advances do lead to greater investment in renewable technologies. Though patents are an imperfect measure of the innovative performance of a country, it is still considered as a relevant measure of technological innovation (Johnstone et al. 2010).

(5) Access to finance plays a crucial role in renewable energy development. Investment in renewable energy requires large upfront costs. We capture this by using a financial development variable that measures the share of domestic credit to the private sector. It's the same variable used by Brunnschweiler (2010) in examining the role of financial development on renewable energy development.

(6) To control for the impact of education on renewable energy development, we use secondary school enrollment as a proxy for human capital (Pfeiffer and Mulder 2013; Zhao et al. 2013). Venturing into renewable energy is knowledge or technology intensive and this can be facilitated by a certain level of human capital development.

(7) Foreign direct investments (FDI) and official development assistance (ODA) are two external sources of funding, which may potentially influence the adoption of renewable energy in developing countries. This is measured as a share of GDP.

(8) One of the first key steps in attempting to control global carbon emissions was the adoption of Kyoto protocol in late 1997. We control the impact of Kyoto protocol by introducing a time dummy from 1998 onwards.

(9) Renewable energy policies facilitate the adoption of renewable energy in developing countries. According to Pfeiffer and Mulder (2013) developing countries are increasingly implementing policies promoting renewable energy even though there are no binding agreements for them to reduce emissions. The IEA complies several policy types related to renewable energy including economic instruments, information and education, policy support, regulatory instruments, research and development and voluntary approaches (IEA 2014). We use a dummy variable if developing countries implement any of these policies.

(10) We include hydropower energy as an additional control variable. We suspect that countries having large hydropower may not be as keen in investing in new renewable energies as opposed those who do not have it. Alternatively, it could also be possible that they are more enthusiastic in adopting nonhydro energy given they already have the experience in dealing with renewable energies.

(11) To take into account the traditional sources of energy, we include in the analysis the country's production of coal and crude oil (Marques et al. 2010). We want to include local production of gas but data is only available starting 1990, hence we dropped it. Countries that have a relatively higher production of these traditional sources may be reluctant to invest in renewable energy. If renewable energy complements traditional sources, then an increase in coal and oil production is positively associated with renewable energy; while if it substitutes, we expect the opposite.

(12) We include crude oil price to capture the impact of market prices on renewables. Since we are using the world price for crude oil, these prices vary only with time and 
not across countries. It would have been ideal if we could have used the local prices for conventional fossil fuels in each developing country but unfortunately, that is not addressed in this current paper because of limited data availability.

(13) Aside from regional dummies, we also include in the analysis coastal dummy taking value of 1 if a country has a coastal area and 0 otherwise. This serves as proxy for generation potential of renewable energy or ease of trading because of its accessibility.

(14)Lastly, we control for time variations by including year dummies in the regression.

We try to capture several relevant variables that may potentially influence the diversification of nonhydro renewable energy in developing countries. However, we still cannot discount the fact that there might be other variables not included in the analysis that may influence the diversification. Tables 4.1 and 4.2 provide the data descriptions and summary statistics of the variables used in this study.

Table 4.1. Data descriptions.

\begin{tabular}{|c|c|c|}
\hline Variable & Definition & Source \\
\hline \multicolumn{3}{|l|}{ Dependent variables } \\
\hline Number of nonhydro & $\begin{array}{l}\text { Number of nonhydro renewable sources (wind, solar, } \\
\text { geothermal, waste and biomass) }\end{array}$ & EIA \\
\hline Diversity index & $\begin{array}{l}\text { Takes value } 1 \text { if a country adopts only } 1 \text { nonhydro source } \\
\text { and converges to total number of nonhydro sources if each } \\
\text { source generates electricity equally. If a country has not } \\
\text { adopted any nonhydro sources, its value is zero }\end{array}$ & $\begin{array}{l}\text { Own } \\
\text { computation }\end{array}$ \\
\hline $\begin{array}{l}\text { Nonhydro energy } \\
\text { generation }\end{array}$ & $\begin{array}{l}\text { Total generation of nonhydro renewable electricity in } \\
\text { billion kilowatt-hours per } 1 \text { million people }\end{array}$ & EIA \\
\hline \multicolumn{3}{|l|}{ Independent variables } \\
\hline GDP per capita & GDP per capita in constant 2005 USD & WDI \\
\hline Energy import & Net energy imports (\% of energy use) & WDI \\
\hline Population growth & Annual population growth in $(\%)$ & WDI \\
\hline Patents & Total patent application & WDI \\
\hline Access to finance & Domestic credit to private sector (\% of GDP) & WDI \\
\hline Secondary enrollment & Secondary school enrollment (\% gross) & WDI \\
\hline FDI & Foreign direct investment, net inflows (\% of GDP) & WDI \\
\hline ODA & Net official development assistance received (\% of GNI) & WDI \\
\hline Crude oil price & Crude oil prices (West Texas intermediate) & $\mathrm{BP}$ \\
\hline Kyoto protocol & Dummy variable takes value of 1 from 1998 onwards & IEA \\
\hline Renewable policy & $\begin{array}{l}\text { Dummy variable takes value of } 1 \text { from the year of } \\
\text { implementation of a renewable energy policy }\end{array}$ & IEA \\
\hline Hydro energy & $\begin{array}{l}\text { Total hydroelectric power generated in billion kilowatt- } \\
\text { hours / } 1 \text { million people }\end{array}$ & EIA \\
\hline Oil production & Total oil production in thousands barrel / 1 thousand people & EIA \\
\hline Coal production & Total coal production in thousand tons / 1 thousand people & EIA \\
\hline Coastal & Dummy variable takes a value of 1 if a country has a coast & Google map \\
\hline
\end{tabular}


Table 4.2. Descriptive statistics for developing countries from 1980 to 2011.

\begin{tabular}{lrrrrr}
\hline \multicolumn{1}{c}{ Variable } & Obs & \multicolumn{1}{c}{ Mean } & Std. Dev. & Min & \multicolumn{1}{c}{ Max } \\
\hline & & & & & \\
Dependent variables & & & & 0 & 4 \\
Number of nonhydro & 3946 & 0.53 & 0.90 & 0 & 2.89 \\
Diversity index & 3946 & 0.36 & 0.56 & 0 & 0.67 \\
Nonhydro energy & 3946 & 0.01 & 0.05 & & \\
& & & & & \\
Independent variables & & & & & \\
GDP per capita & 4087 & 2154.27 & 2159.19 & 50.04 & 14777.22 \\
Energy import & 2688 & -41.92 & 195.54 & -1982.88 & 99.96 \\
Population growth & 4727 & 1.81 & 1.35 & -10.96 & 11.18 \\
Patents & 1473 & 6.25 & 1.93 & 0.69 & 13.17 \\
Financial dev & 3707 & 28.59 & 24.60 & 0.56 & 167.54 \\
Secondary enrollment & 2944 & 54.79 & 30.03 & 2.48 & 122.20 \\
FDI & 3849 & 3.69 & 11.79 & -82.93 & 366.36 \\
ODA & 3654 & 9.29 & 12.95 & -2.70 & 242.29 \\
Kyoto protocol & 4752 & 0.45 & 0.50 & 0 & 1 \\
Renewable policy & 4752 & 0.10 & 0.30 & 0 & 1 \\
Hydro energy & 4070 & 0.37 & 0.89 & 0 & 10.08 \\
Oil production & 3927 & 4.23 & 13.93 & 0 & 133.73 \\
Coal production & 4142 & 0.29 & 1.01 & 0 & 12.55 \\
Crude oil price & 4752 & 37.66 & 25.00 & 14.39 & 100.06 \\
Coastal & 4752 & 0.78 & 0.42 & 0 & 1 \\
\hline Souc: Aut & & & &
\end{tabular}

Source: Author's calculations based on the data described in Table 4.1

\subsubsection{Empirical analysis}

In order to investigate what drives the diversification of nonhydro sources of renewable energy in developing countries, we specify the main regression analysis as follows:

$$
d_{i t}=\alpha+\beta_{1} G D P_{i t}+\delta \boldsymbol{X}_{i t}+u_{i}+v_{t}+\varepsilon_{i t}
$$

where $d_{i t}$ captures the diversification of nonhydro sources renewable energy adopted in country $i$ in year $t, G D P_{i t}$ captures the income per capita, $X_{i t}$ is the set of control variables capturing energy import, access to finance, technological innovation, renewable energy policies, education, Kyoto protocol, external sources of funding and other relevant variables that may influence diversification, $u_{i}$ is the country fixed effects used to capture time-invariant country heterogeneity, $v_{t}$ is time fixed effects and $\varepsilon_{i t}$ is the remaining error capturing all other unobservable factors that might influence diversification.

Our main dependent variable captures the diversity in nonhydro source of renewable energy and is measured in two ways. First, we use the number of nonhydro technologies the country adopts and second, we use our proposed diversity index or simply the weighted number of nonhydro sources. The first measure of diversification is a count. Count data models such as Poisson and negative binomial have been suggested for estimating the number 
of occurrences of an event count (Cameron and Trivedi 1998). For this paper, an event count refers to the number of nonhydro sources adopted by developing countries. Following Grogger and Carson (1991), the basic Poisson model can be written as follows:

$$
\operatorname{Pr}\left(Y_{i}=j\right)=\frac{\exp (-\lambda) \lambda^{j}}{j !}
$$

where there are $i=1,2, \ldots, n$ observations, $Y_{i}$ is the $i$ th observation on the count variable, $j=$ $0,1,2,3,4$ are the possible values of $Y_{i}$ which refers the number of nonhydro sources of renewable energy and $\lambda$ is the Poisson parameter to be estimated. A restrictive property of the Poisson model is the assumption that the conditional mean of $Y_{i}$ is equal to the conditional variance, that is,

$$
E\left(Y_{i} \mid X_{i}\right)=\operatorname{Var}\left(Y_{i} \mid X_{i}\right)=\lambda_{i}=\exp \left(X_{i} \beta\right)
$$

This assumption of mean-variance equality in the Poisson distribution is not realistic and often problematic since in most cases when using actual data, the conditional variance often exceeds the conditional mean resulting in an over-dispersion problem (Cameron and Trivedi 1998). In the presence of over-dispersion, the conditional mean is still consistent when estimating using the Poisson model but the standard errors of $\beta$ are biased downward (Cameron and Trivedi 1986; Grogger and Carson 1991). To account for this over-dispersion problem, we use negative binomial distribution written as follows:

$$
\operatorname{Pr}\left(Y_{i}=j\right)=\frac{\Gamma\left(j+\frac{1}{\alpha}\right)}{\Gamma(j+1) \Gamma\left(\frac{1}{\alpha}\right)}\left(\alpha \lambda_{i}\right)^{j}\left[1+\alpha \lambda_{i}\right]^{-\left(j+\frac{1}{\alpha}\right)}
$$

where $\alpha>0$ is a nuisance parameter, and $\Gamma$ is the gamma function, i.e. a discrete probability density function for $j$. The first two moments of the negative binomial are given by

$$
E\left(Y_{i} \mid X_{i}\right)=\lambda_{i}=\exp \left(X_{i} \beta\right)
$$

and

$$
\operatorname{Var}\left(Y_{i} \mid X_{i}\right)=\lambda_{i}\left(1+\alpha \lambda_{i}\right)
$$

With the negative binomial, the restrictive assumption of mean-variance equality is relaxed because $\operatorname{Var}\left(Y_{i} \mid X_{i}\right)$ is greater than $E\left(Y_{i} \mid X_{i}\right)$. When $\alpha \rightarrow 0$, the gamma distribution converges to the Poission distribution (Cameron and Trivedi 1986; Grogger and Carson 1991).

The negative binomial distribution can be regarded as a generalization of the Poisson distribution with an additional parameter allowing the variance to exceed the mean (Allison and Walterman 2002). Figure 4.1 shows the distribution of the number of nonhydro sources of renewable energy and Figure 4.2 shows how well our dependent variable fits on the negative binomial distribution over the Poisson distribution. Hence, negative binomial regression is preferred over the Poisson one because over-dispersion is present in our data set (Figure 4.2). 


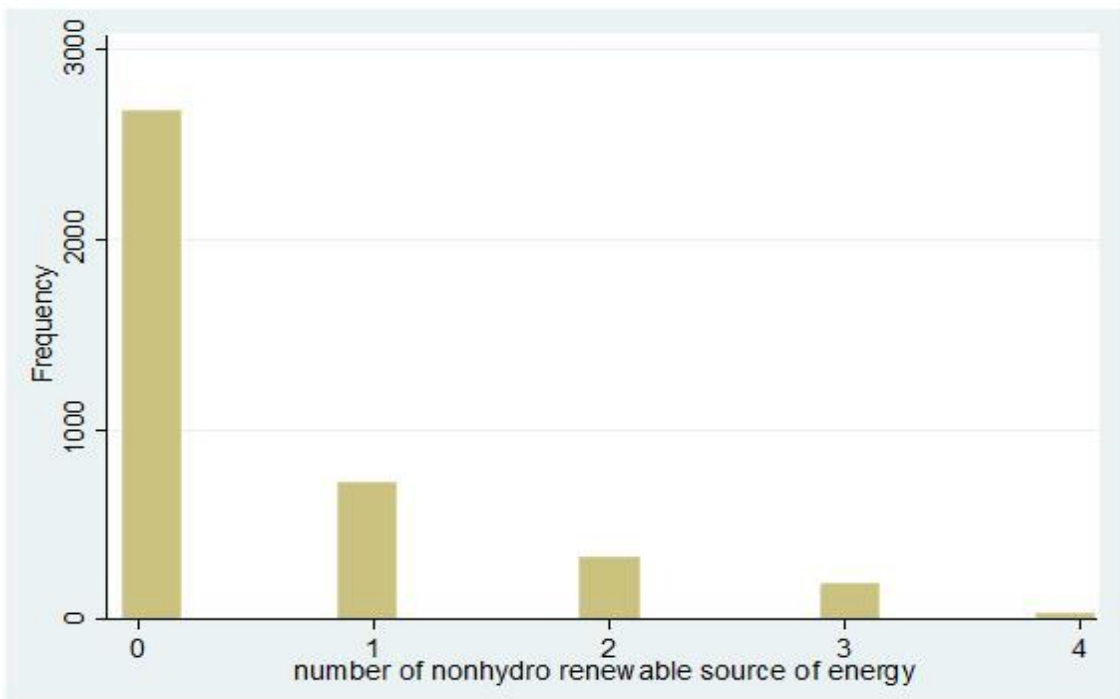

Figure 4.2. Distribution of nonhydro sources of renewable energy.

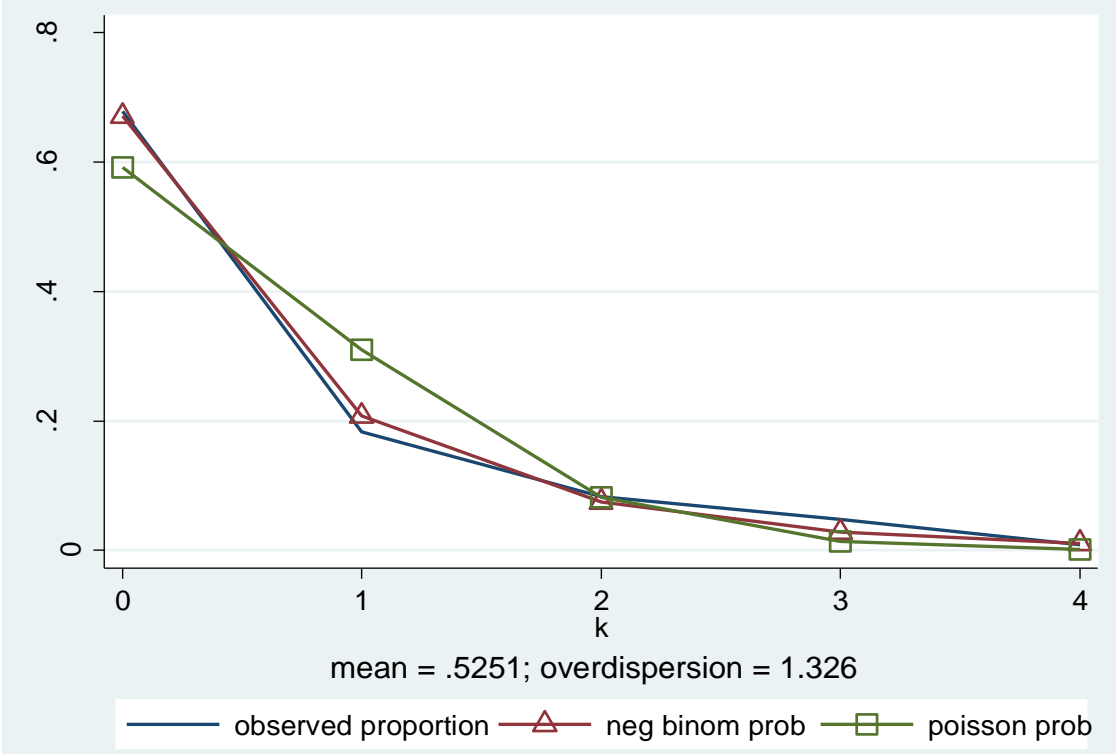

Figure 4.3. Goodness of fit of Poisson and negative binomial distribution.

Although the negative binomial has an extension in Stata for analyzing panel data, we refrain from doing so because Allison and Walterman (2002) demonstrated that the conditional negative binomial model for panel data is not a true fixed-effect because it does not control for all time invariant covariates. Guimarães (2008) also asserted this claim and showed that the conditional fixed effects negative binomial model for count panel data does not control for individual fixed effects. Hence, we estimate the unconditional estimation of a fixed effects negative binomial model by including regional and time fixed effects. 
In our next step, we use the derived diversity index as another measure of nonhydro diversification. Notice that this variable has a substantial number of zero observations (for those developing countries that are yet to invest in any nonhydro sources) and positive continuous values starting from 1 up to 2.89 (see Table 4.2). To deal with this issue, there are two alternative approaches available in the literature that has been vigorously debated: the Heckman's two-stage sample selection model, usually called the Heckit or Heckman model and the other is called two-part model (TPM) (Manning et al. 1987; Leung and Yu 1996). The striking difference between the two methods is the inclusion of the inverse Mills ratio as an additional regressor for the Heckit model to control for potential selection bias. The relative merits of the two models have been vigorously debated in the literature (Hay and Olsen 1984; Manning et al. 1987; Leung and Yu 1996; Dow and Norton 2003). A more substantive consideration in choosing between the two models is on the treatment of zero observation as discussed by Dow and Norton (2003) and as highlighted by Frondel and Vance (2010). A zero observation can either mean (1) an actual outcome that is a true observable zero or (2) a potential outcome arising from missing observation and latent variables that are only partially observed. The latter captures the selection bias and requires the use of the Heckman selection model while the former emphasizes that there is no selection bias when modeling true zeros or actual outcome (Dow and Norton 2003). Based on this argument, we prefer to use the method of the two-part model (TPM) pioneered by Duan et al. (1983) assuming that the zero observations are actual outcomes referring to the countries that have not invested in any nonhydro source of renewable energy ${ }^{23}$.

The two-part model decomposes an observed random variable into two observed variables. It consists of two equations. The first equation estimates the entire sample with zero values and a positive outcome while the second equation analyzes the subset of the sample with a positive outcome. The first stage models the choice of whether to adopt nonhydro renewable energy or not and the second stage, conditional on deciding to adopt, models the diversification of nonhydro sources of renewable energy in developing countries. We specify the first equation as follows:

$$
\operatorname{Pr}\left[\operatorname{dre}_{i t}>0 \mid X_{i t}\right]=\phi\left(\beta X_{i t}+\varepsilon_{i t}\right)
$$

where $d r e_{i t}$ is a dummy variable taking the value of 1 if the number of nonhydro resources adopted in country $i$ at time $t$ is greater than zero and zero otherwise. $X_{i t}$ is the vector of control variables, $\beta$ is the associated coefficient, $\phi$ is the standard normal distribution and $\varepsilon_{\text {it }}$ is the remaining error term. For the second equation, we investigate diversification as follows:

$$
d r e_{i t}=\beta X_{i t}+\omega_{i t}
$$

\footnotetext{
${ }^{23}$ We also acknowledge that some zero observations might be missing observations reflecting the potential renewable energy generation especially those off-grid electricity generations not included in the IEA database. However even if one strongly argues favoring that zero observations are not actual zeros in our empirical analysis, it is likely that there will be no striking difference in the estimates between the two models because Pfeiffer and Mulder (2013) used both the Heckman sample selection and TPM in their analysis found similar results.
} 
where $d r e_{i t}$ captures how diversified the nonhydro renewable energy sources adopted by a particular country $i$ at a given time $t i s, X_{i t}$ captures the independent variables and $\omega_{i t}$ is the remaining error. We estimate equation 4.11 and 4.12 using the methods of logit and ordinary least squares, respectively.

The expected value of diversification $\mathrm{E}[$ dre $\mid \mathrm{X}]$ consists of two parts. The first part resulting from the first stage, $(\operatorname{Pr}[d r e>0 \mid \mathrm{X}]$, which is observing the probability of whether developing countries decide to invest in any nonhydro sources or not and the second part, $\mathrm{E}[$ dre $\mid$ dre $>0, \mathrm{X}]$ conditional on adopting, captures the determinants of diversification. Therefore, the combine marginal effect is given as follows:

$$
E[d r e \mid X]=\operatorname{Pr}[d r e>0 \mid X] * E[d r e \mid d r e>0, X]
$$

Based on equation 4.13, the general formula for deriving the average marginal effect of the independent variables, $\mathrm{X}$, on the nonhydro diversification can be obtained as follows ${ }^{24}$ :

$$
\begin{aligned}
& \frac{E[\text { dre }]}{\partial x}=\frac{\operatorname{Pr}[\text { dre }>0] * E[\text { dre } \mid \text { dre }>0]}{\partial x} \\
& \frac{E[\text { dre }]}{\partial x}=\left(\operatorname{Pr}[\text { dre }>0] * \frac{\partial E[\text { dre } \mid \text { dre }>0]}{\partial x}\right)+\left(E[\text { dre } \mid \text { dre }>0] * \frac{\partial \operatorname{Pr}[\text { dre }>0]}{\partial x}\right)
\end{aligned}
$$

For further analysis and robustness check, we use another estimation technique suggested in the literature that can handle a substantial number of zero observations. We use the Poisson pseudo-maximum likelihood (PPML) estimation technique proposed by Santos Silva and Tenreyro (2006). Aside from dealing with zero observations, the PPML approach gives consistent estimates in the presence of heteroskedasticity (Santos Silva and Tenreyro 2006; Martínez-Zarzoso et al. 2007). This is subsequently confirmed by Santos Silva and Tenreyro (2011) and Martínez-Zarzoso (2013) that PPML is consistent in the presence of heteroskedasticity and is well-behaved when the presence of zero observations is quite large. The diversification or adoption of nonhydro sources of renewable energy can be modeled by using an exponential function as follows:

$$
E\left(n h e_{i} \mid x_{i}\right)=\alpha_{i} \exp \left(x_{i} \tilde{\beta}\right)
$$

and $\beta$ can be estimated by solving a set of first-order conditions:

$$
\sum_{i=1}^{n}\left[n h e_{i}-\exp \left(x_{i} \tilde{\beta}\right)\right] x_{i}=0
$$

where $n h e_{i}$ is the dependent variable capturing either the diversification index or the generation of nonhydro energy measured in billion kilowatt-hours per thousand people, $x_{i}$ is the set of independent variables and $\beta$ are the parameters to be estimated. PPML assumes a non-negative energy generation and is consistent and well-behaved in the presence of

\footnotetext{
${ }^{24}$ For further discussion on the merits of the two-parts model, please refer to the works of Manning et al. (1987), Leung and Yu (1996), Dow and Norton (2003), Frondel and Vance (2012).
} 
substantial number of zero observations. In addition, the dependent variable does not necessarily be an integer and the distribution does not need to follow a Poisson distribution (Santos Silva and Tenreyro 2006; Martínez-Zarzoso et al. 2007).

\subsection{Results and Discussions}

Despite relatively huge upfront investment cost in renewable energy, there is a rising penetration of renewable energy technology in developing countries. Before we present the results of our empirical analysis, we begin by presenting some graphical evidence of the rising adoption and diversification of nonhydro sources of renewable energy in developing countries. We observe a gradual increase in the adoption of nonhydro sources from 1980 up to 1995 but from 1995 to 2010, we observe a rather dramatic increase in the adoption of nonhydro sources particularly in wind, solar, biomass and waste technology (Figure 4.4). This dramatic increase of the adoption of renewable energy after 1995 was possibly influenced by the adoption of Kyoto protocol in late 1997. Although there is no binding commitment for developing countries to reduce emissions, many developing countries were venturing into clean development mechanism (CDM) projects. The benefits that they get from CDM projects include investments in climate change mitigation, transfer to climate friendly technologies as well as improvement in the livelihood (UNFCCC 2014). This gives developing countries incentives to adopt nonhydro sources of renewable energy.

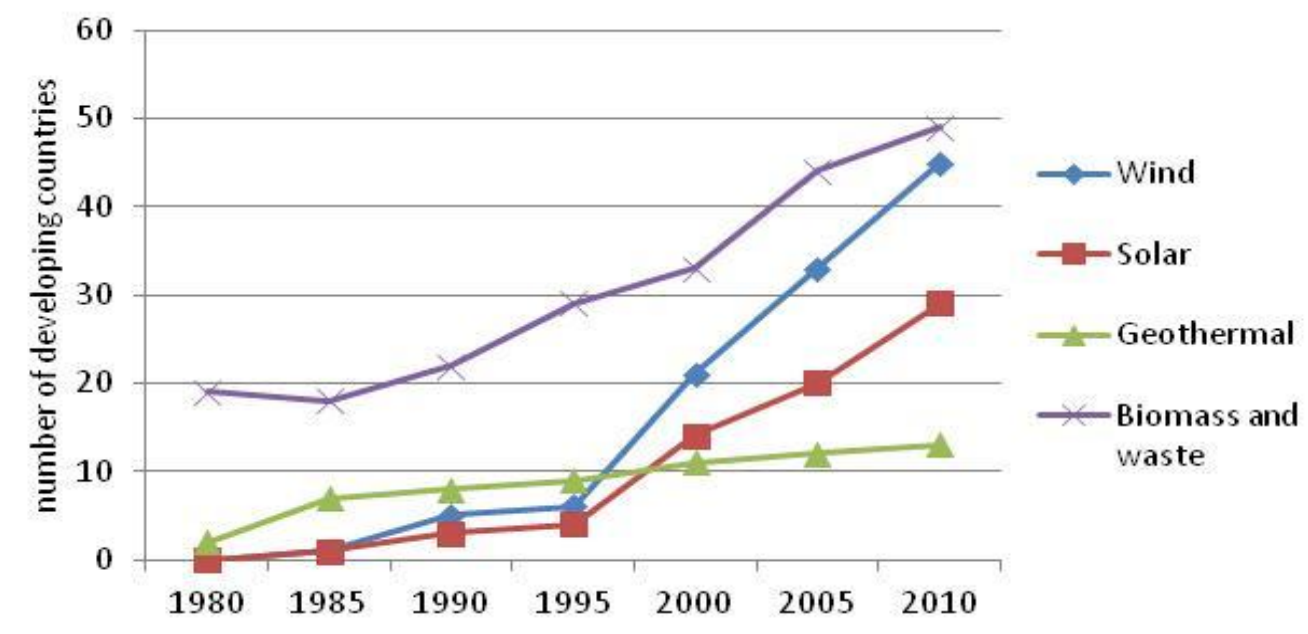

Figure 4.4. Number of countries adopting nonhydro sources of renewable energy.

Although we document a rising trend in the number of developing countries adopting various sources of nonhydro renewable energy, a substantial number of them are still not investing into any of these sources. Figure 4.5 shows that in 2010 roughly close to seventy 
developing countries were reported as having no investment in any of the available nonhydro sources of renewable energy. However, we observed a steady decline in the number of developing countries not investing in any of these nonhydro sources of renewable energy. More developing countries are starting to integrate renewable energy in their energy system.

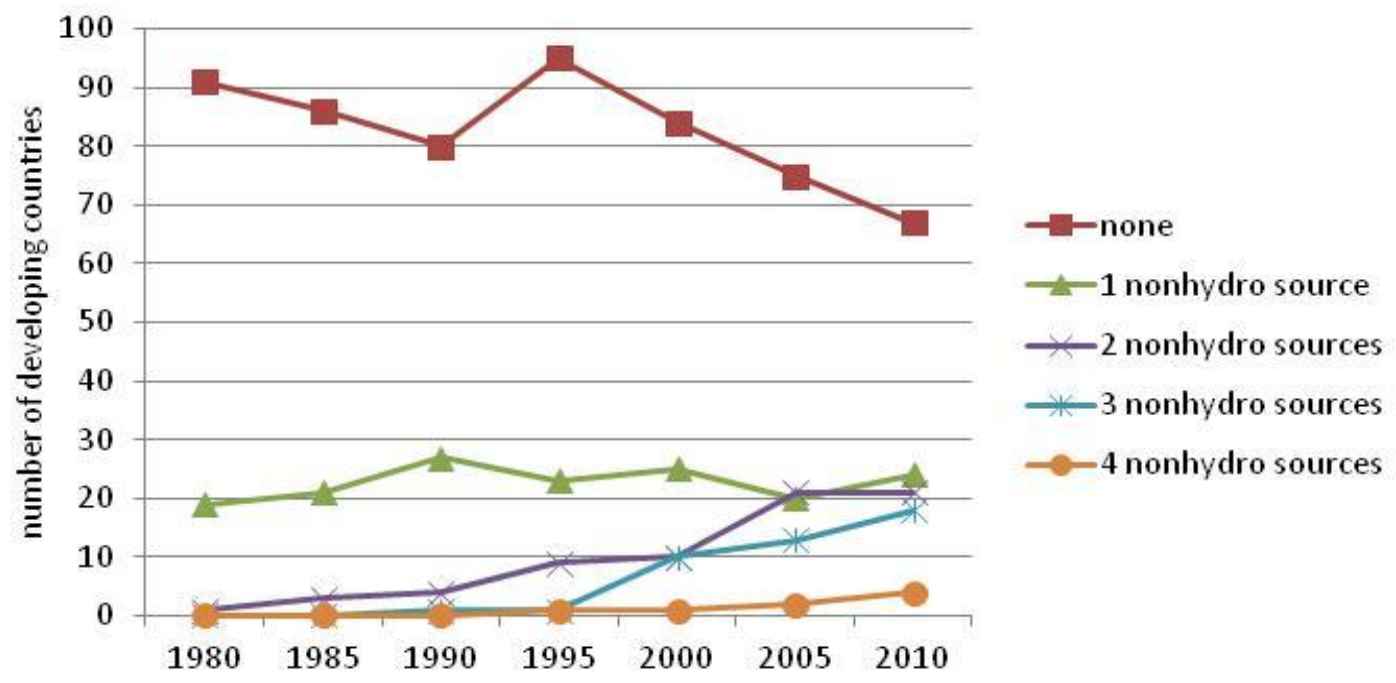

Figure 4.5. Number of countries diversifying sources of renewable energy.

\subsubsection{Baseline results}

This section presents evidence on the factors that promote or hinder the diversification of nonhydro sources. Table 4.3 presents the baseline results considering different specifications while controlling for time and regional fixed effects. The dependent variable is the number of nonhydro sources of renewable energy adopted by developing countries. Hence, our preferred estimation is the negative binomial regression considering the count nature of our dependent variable ${ }^{25}$. Having a large observation with zero values will potentially create bias in our estimates if we use the ordinary least squares (OLS). Nevertheless, we also provide the estimation result from the pooled OLS for comparison. Since negative binomial models the log of the expected count, we can interpret the coefficients as follows: for a one unit change in the independent variable, the log count of the nonhydro sources is expected to change by $\beta$. Another option of measuring the effect of the independent variables on the number of nonhydro sources is through the incidence rate ratio (IRR). The IRR represents the change in the dependent variable in terms of percentage increase (IRR > 1) or decrease (IRR $<1)$. This is done by exponentiating the regression coefficient. For example, a one unit

\footnotetext{
${ }^{25}$ We refrain from using a fixed effects negative binomial estimator in accounting for the unobserved time invariant heterogeneity because of the methodological problems associated with this (Allison and Walterman 2002). We wanted to use a country dummy but the estimation fails to converge, instead we control for region specific dummy variables. We also control for variations across time by including year dummies in the analysis.
} 
change in the independent variable is associated with a $[\exp (\beta)-1]^{*} 100$ percentage change in the dependent variable in the log form (Cameron and Trivedi 2010; Tran et al. 2013) ${ }^{26}$.

Results show that income plays a positive significant role in explaining diversification. Higher incomes make developing countries more capable of diversifying their nonhydro energy sources. However, its effect diminishes with the inclusion of policy variables and even losses it significance with the inclusion of the patent variable and other sources of external funding. This implies that adoption of renewable energy is not only influenced by income but is complemented with technology and policy. While the effect of income on renewable energy has been well documented in the literature, none of the studies explored the nonlinear effect of income (Vachon and Menz 2006; Marques et al. 2010; Pfeiffer and Mulder 2013; Zhao et al. 2013; Aguirre and Ibikunle 2014). Our results showed evidence that income has a significant nonlinear influence on the number of nonhydro sources depicting a U-shaped type of relationship. This suggests that as developing countries grow, the diversification of renewable energy sources declines, but as the economy develops, eventually diversification of nonhydro sources of renewable energy will follow. The turning point is around US $\$ 1,900$ to US $\$ 2,400$ slightly above the average per capita income in developing countries. This has an optimistic implication because it means that developing countries will soon start to invest in various sources of nonhydro renewable energy.

In all cases, the coefficient of policy variable is positive and significant. Various government policies have been introduced to stimulate the adoption of renewable energy. Notice that once policy variable is included in the regression, the magnitude of the income coefficient declines. This holds true in all the succeeding analysis. This implies that policy support is necessary to encourage stakeholders to invest into renewable due to several barriers to adoption. This affirms the argument of Dulal et al. (2013) that political will or government intervention is necessary for countries to move toward renewable because relying on the market alone is insufficient. Thus, implementing policies that promotes the adoption of renewable energy provide incentives for developing countries to diversify sources of nonhydro renewable energy. This results accords well with the findings of Popp et al. (2011) suggesting that renewable is not driven by demand but rather by policy because renewable energy are more expensive than fossil fuels. Developing countries with policies supporting renewable energy translate to a $36 \%$ percent in the log count of nonhydro sources, holding other factors constant. This also confirms the findings of Pfeiffer and Mulder (2013) showing that countries that implemented economic or regulatory policies promoting renewable energy are more likely to invest in nonhydro technology. Collier and Venables (2012) also pointed out that one of the factors hindering Africa in adopting green technologies is the absence of governance capacity, which relates to the implementation of policies promoting renewable energy. However, Zhao et al. (2013) warn about policy crowdedness, wherein the effectiveness of policies diminish as more renewable energy policies are put in place.

\footnotetext{
${ }^{26}$ For convenience in discussing the associated relationships, we present in the discussions the regression coefficients of the negative binomial while the corresponding IRR is available in the appendix section.
} 
Population growth, energy imports and oil prices positively support diversification. A growing population is coupled with rising energy demand. Nonhydro renewable energy can supplement this rising energy need and this could explain why sources of nonhydro renewable energy are more diversified with a rising population. Countries that are more dependent on foreign sources of energy are more likely to diversify sources of nonhydro renewable energy and a higher market price for crude oil price facilitates diversification. Energy security is becoming more of a concern to policy makers and to citizens in developing countries. In this regard, renewable energy presents an attractive option in improving energy security. Though we observe a positive association with diversification and energy security, its effect is rather small. A $1 \%$ increase in energy import will only translate to $0.2 \%$ in the $\log$ count of nonhydro sources of renewable energy. While Marques et al. (2010) and Aguirre and Ibikunle (2014) suggest that energy prices are not relevant factors in explaining the diffusion of renewable energy, we argued the opposite. The effect of an increasing world price for crude oil is positively correlated to the diversification of nonhydro sources of renewable energy. Our results are consistent with Chang et al. (2009) that showed increases in fossil fuel prices are associated with increases in renewable energy use.

We use the number of patents as a proxy for technological innovation and found that its effect is positive and significant suggesting technological innovations lead to a greater investment as well as diversification in nonhydro sources of renewable energy. In relation to this, adoption of renewable energy requires a certain level of knowledge and skills. We also find evidence showing that the improvements in human capital as measured by secondary enrollment positively contribute to the diversification. In addition, access to finance facilitates diversification because investments in renewable energy require a high level of financing. Having access to finance will help promote diversification of nonhydro renewable energy. Painuly and Wohlgemuth (2006) noted that the absence of well-developed financial intermediaries and the consequent financing difficulties impede the development of renewable energy in developing countries. With this, our results largely confirmed previous findings on the influence of technology (Popp et al. 2011), improvement in human capital (Pfeiffer and Mulder 2013) and access to finance (Brunnschweiler 2010) on renewable energy. This further confirms what was highlighted by Collier and Venables (2012) that developing countries with more technology, available human capital and skills and developed financial markets can go into renewable energies. Our results add to this strand of literature by presenting evidence that these factors also contribute to the diversification of nonhydro sources of renewable energy.

On the contrary, high levels of energy generated from hydropower do not promote the diversification of nonhydro sources of renewable energy. This shows that developing countries having a relatively high production of hydropower are not enthusiastic in adopting other sources of renewable energy. This confirms previous findings showing that countries having relatively low carbon intensity are likely to diminish incentives to invest in renewable energy (Popp et al. 2011; Pfeiffer and Mulder 2013). Similarly, abundance of oil in developing countries decreases the attractiveness in investing to other alternative sources of energy. Local oil production reduces the concern of energy security, increases the relative price of other renewable energy and undermines support for reducing emissions (Popp et al. 
2011) making investment in renewable energy unattractive. However, coal production posted a significant positive association with diversification. This is quite the opposite of what we were expecting. We expect that abundance of coal reduce the incentive to invest in renewable energy. One possible explanation for this relates to the global effort in curbing carbon emissions. Though there are no commitments to reduce emissions from developing countries, those who are more reliant on traditional sources of energy will experience increasing pressure to produce cleanly, thus they are incline to adopt nonhydro renewable energy sources. For example, in China and India where fossil fuels are still used in large volumes, investments in renewable energy technologies have been made (Aguirre and Ibikunle 2014). Another possible explanation for this positive association of coal on diversification is that perhaps coal complements nonhydro energy but this merits further investigation.

Other control variables included in the regression are two sources of external funding; foreign direct investment (FDI) and official development assistance (ODA). Both are measured as a percentage to the countries' income. Results show that the coefficient of FDI and ODA are negative and significant. The same findings were reported by Pfeiffer and Mulder (2013). While these two external sources of funding are important for technology transfers, neither of them facilitates the diversification of nonhydro sources. This suggests that aid dependent countries find it hard to move into renewables and investments on these technologies may not be the priority of the donors. Another possible explanation for this is that our ODA measure does not reflect the aid for the renewable energy sector. The ODA variable used in this study is aggregate aid and does not capture the energy specific ODA due to data limitations. Similarly, FDI does not promote diversification of nonhydro sources of renewable energy in developing countries. This is because most FDI goes into oil industries and not into renewables. For example, in Africa investments in extractive industries remain the most important driver of FDI (UNCTAD, 2013). This means limited investments directly help the renewable energy sector.

Since our estimation fails to converge when using country fixed effects as the model includes too many dummy variables, we instead use regional fixed effects. We also incorporate in the analysis year dummies to control for variations related with time ${ }^{27}$. For regional fixed effects, developing countries were divided into six regions, namely: (1) Latin America and the Caribbean, (2) Europe and Central Asia, (3) Middle East and North Africa, (4) Sub-Sahara Africa, (5) South Asia and (6) East Asia and Pacific. Aside from the regional classification, we also control for geographic endowments of countries taking a value of 1 if the country has coast and 0 otherwise. This controls the geographic advantage of some countries in harnessing renewable energy especially wind and wave energy. The effect of the coastal dummy is robust across specifications suggesting that developing countries endowed with coastal areas are more likely to diversify nonhydro sources of renewable energy as compared to landlocked countries. Coastal areas are relatively windy making it an ideal location to invest for technologies harnessing wind energy. Having coastal areas also provide ease of access for trade and technology transfer.

\footnotetext{
${ }^{27}$ Estimates of regional and time dummies are not shown to save space but are available upon request.
} 
Table 4.3.Baseline analysis using a negative binomial regression.

\begin{tabular}{|c|c|c|c|c|c|c|c|}
\hline VARIABLES & $\begin{array}{c}(1) \\
\text { OLS }\end{array}$ & $\begin{array}{c}(2) \\
\text { Negbin } \\
\end{array}$ & $\begin{array}{c}(3) \\
\text { Negbin } \\
\end{array}$ & $\begin{array}{c}(4) \\
\text { Negbin } \\
\end{array}$ & $\begin{array}{c}(5) \\
\text { Negbin } \\
\end{array}$ & $\begin{array}{c}(6) \\
\text { Negbin } \\
\end{array}$ & $\begin{array}{c}(7) \\
\text { Negbin } \\
\end{array}$ \\
\hline $\log$ GDP pc & $\begin{array}{l}0.088 * * \\
(0.0421)\end{array}$ & $\begin{array}{c}0.224 * * * \\
(0.0642)\end{array}$ & $\begin{array}{c}0.107 * \\
(0.0595)\end{array}$ & $\begin{array}{c}0.010 \\
(0.0693)\end{array}$ & $\begin{array}{c}-0.047 \\
(0.0680)\end{array}$ & $\begin{array}{c}-2.050 * * * \\
(0.4675)\end{array}$ & $\begin{array}{c}-1.899 * * * \\
(0.4586)\end{array}$ \\
\hline $\log$ GDP pc_sq & & & & & & $\begin{array}{c}0.136 * * * \\
(0.0305)\end{array}$ & $\begin{array}{c}0.122 * * * \\
(0.0297)\end{array}$ \\
\hline energy import & $\begin{array}{l}0.00005 \\
(0.0001)\end{array}$ & $\begin{array}{l}0.001 * * \\
(0.0004)\end{array}$ & $\begin{array}{c}0.002 * * * \\
(0.0005)\end{array}$ & $\begin{array}{c}0.002 * * * \\
(0.0006)\end{array}$ & $\begin{array}{c}0.002 * * * \\
(0.0006)\end{array}$ & $\begin{array}{l}0.001 * * \\
(0.0006)\end{array}$ & $\begin{array}{c}0.002 * * * \\
(0.0006)\end{array}$ \\
\hline population growth & $\begin{array}{c}0.083 * * * \\
(0.0282)\end{array}$ & $\begin{array}{c}0.011 \\
(0.0500)\end{array}$ & $\begin{array}{c}0.067 \\
(0.0445)\end{array}$ & $\begin{array}{c}0.135 * * * \\
(0.0477)\end{array}$ & $\begin{array}{c}0.168 * * * \\
(0.0432)\end{array}$ & $\begin{array}{l}0.123 * * \\
(0.0496)\end{array}$ & $\begin{array}{c}0.155 * * * \\
(0.0450)\end{array}$ \\
\hline oil price & $\begin{array}{c}0.018 * * * \\
(0.0043)\end{array}$ & $\begin{array}{c}0.022 * * * \\
(0.0036)\end{array}$ & $\begin{array}{c}0.017 * * * \\
(0.0035)\end{array}$ & $\begin{array}{c}0.024 * * * \\
(0.0035)\end{array}$ & $\begin{array}{c}0.021 * * * \\
(0.0034)\end{array}$ & $\begin{array}{c}0.023 * * * \\
(0.0037)\end{array}$ & $\begin{array}{c}0.020 * * * \\
(0.0035)\end{array}$ \\
\hline $\log$ finance dev & $\begin{array}{l}0.061 * * \\
(0.0264)\end{array}$ & $\begin{array}{c}0.166 * * * \\
(0.0437)\end{array}$ & $\begin{array}{c}0.186 * * * \\
(0.0415)\end{array}$ & $\begin{array}{l}0.118 * * \\
(0.0540)\end{array}$ & $\begin{array}{c}0.140 * * * \\
(0.0529)\end{array}$ & $\begin{array}{c}0.143 * * * \\
(0.0521)\end{array}$ & $\begin{array}{c}0.163 * * * \\
(0.0512)\end{array}$ \\
\hline ren policy & $\begin{array}{c}0.550 * * * \\
(0.0928)\end{array}$ & & $\begin{array}{c}0.557 * * * \\
(0.0698)\end{array}$ & & $\begin{array}{c}0.337 * * * \\
(0.0713)\end{array}$ & & $\begin{array}{c}0.314 * * * \\
(0.0699)\end{array}$ \\
\hline $\log$ patents & $\begin{array}{c}0.075 * * * \\
(0.0086)\end{array}$ & & & $\begin{array}{c}0.083 * * * \\
(0.0127)\end{array}$ & $\begin{array}{c}0.064 * * * \\
(0.0125)\end{array}$ & $\begin{array}{c}0.076 * * * \\
(0.0129)\end{array}$ & $\begin{array}{c}0.059 * * * \\
(0.0127)\end{array}$ \\
\hline sec enrollment & $\begin{array}{c}0.006 * * * \\
(0.0017)\end{array}$ & $\begin{array}{c}0.004 \\
(0.0028)\end{array}$ & $\begin{array}{c}0.003 \\
(0.0027)\end{array}$ & $\begin{array}{c}0.013 * * * \\
(0.0026)\end{array}$ & $\begin{array}{c}0.014 * * * \\
(0.0025)\end{array}$ & $\begin{array}{c}0.014 * * * \\
(0.0027)\end{array}$ & $\begin{array}{c}0.014 * * * \\
(0.0026)\end{array}$ \\
\hline hydro energy & $\begin{array}{c}-0.221 * * * \\
(0.0207)\end{array}$ & $\begin{array}{c}-0.419 * * * \\
(0.0834)\end{array}$ & $\begin{array}{c}-0.313 * * * \\
(0.0786)\end{array}$ & $\begin{array}{c}-0.803 * * * \\
(0.0692)\end{array}$ & $\begin{array}{c}-0.749 * * * \\
(0.0679)\end{array}$ & $\begin{array}{c}-0.844 * * * \\
(0.0716)\end{array}$ & $\begin{array}{c}-0.789 * * * \\
(0.0704)\end{array}$ \\
\hline oil prod'n & $\begin{array}{c}-0.010 * * * \\
(0.0023)\end{array}$ & $\begin{array}{c}-0.014 * * * \\
(0.0052)\end{array}$ & $\begin{array}{l}-0.008 * \\
(0.0050)\end{array}$ & $\begin{array}{c}-0.023 * * \\
(0.0097)\end{array}$ & $\begin{array}{c}-0.019 * * \\
(0.0092)\end{array}$ & $\begin{array}{c}-0.029 * * * \\
(0.0103)\end{array}$ & $\begin{array}{c}-0.025 * * \\
(0.0098)\end{array}$ \\
\hline coal prod'n & $\begin{array}{c}0.011 \\
(0.0228)\end{array}$ & $\begin{array}{c}0.089 * * * \\
(0.0205)\end{array}$ & $\begin{array}{c}0.114 * * * \\
(0.0229)\end{array}$ & $\begin{array}{c}0.080 * * \\
(0.0354)\end{array}$ & $\begin{array}{c}0.105^{* * *} \\
(0.0365)\end{array}$ & $\begin{array}{c}0.098 * * * \\
(0.0365)\end{array}$ & $\begin{array}{c}0.119 * * * \\
(0.0376)\end{array}$ \\
\hline FDI & $\begin{array}{c}-0.026 * * * \\
(0.0066)\end{array}$ & & & $\begin{array}{l}-0.046 * * \\
(0.0223)\end{array}$ & $\begin{array}{c}-0.046 * * \\
(0.0211)\end{array}$ & $\begin{array}{c}-0.050 * * \\
(0.0216)\end{array}$ & $\begin{array}{l}-0.050 * * \\
(0.0206)\end{array}$ \\
\hline ODA & $\begin{array}{c}0.003 \\
(0.0024)\end{array}$ & & & $\begin{array}{l}-0.012 \\
(0.0107)\end{array}$ & $\begin{array}{l}-0.015 \\
(0.0107)\end{array}$ & $\begin{array}{c}-0.023 * * \\
(0.0116)\end{array}$ & $\begin{array}{c}-0.025 * * \\
(0.0114)\end{array}$ \\
\hline coast & $\begin{array}{c}0.343 * * * \\
(0.0554)\end{array}$ & $\begin{array}{c}0.911 * * * \\
(0.1355)\end{array}$ & $\begin{array}{c}0.883 * * * \\
(0.1356)\end{array}$ & $\begin{array}{c}0.952 * * * \\
(0.1489)\end{array}$ & $\begin{array}{c}0.937 * * * \\
(0.1484)\end{array}$ & $\begin{array}{c}0.936 * * * \\
(0.1498)\end{array}$ & $\begin{array}{c}0.921 * * * \\
(0.1487)\end{array}$ \\
\hline $\begin{array}{l}\text { regional dummies } \\
\text { year dummies }\end{array}$ & $\begin{array}{l}\text { yes } \\
\text { yes }\end{array}$ & $\begin{array}{l}\text { yes } \\
\text { yes }\end{array}$ & $\begin{array}{l}\text { yes } \\
\text { yes }\end{array}$ & $\begin{array}{l}\text { yes } \\
\text { yes }\end{array}$ & $\begin{array}{l}\text { yes } \\
\text { yes }\end{array}$ & $\begin{array}{l}\text { yes } \\
\text { yes }\end{array}$ & $\begin{array}{l}\text { yes } \\
\text { yes }\end{array}$ \\
\hline constant & $\begin{array}{c}-2.555 * * * \\
(0.3831)\end{array}$ & $\begin{array}{c}-5.717 * * * \\
(0.5173)\end{array}$ & $\begin{array}{c}-4.774 * * * \\
(0.5031)\end{array}$ & $\begin{array}{c}-5.005^{* * *} * \\
(0.5603)\end{array}$ & $\begin{array}{c}-4.519 * * * \\
(0.5582)\end{array}$ & $\begin{array}{c}2.888 \\
(1.8734)\end{array}$ & $\begin{array}{c}2.563 \\
(1.8407)\end{array}$ \\
\hline $\begin{array}{l}\text { observations } \\
\text { R-squared }\end{array}$ & $\begin{array}{l}1,232 \\
0,553\end{array}$ & 1,386 & 1,386 & 1,232 & 1,232 & 1,232 & 1,232 \\
\hline
\end{tabular}


Aside from considering the number of nonhydro sources of renewable energy adopted by developing countries, we also take into account the total electricity generated from each particular source. We use our derived diversity index as the dependent variable. By using this index, we use the share of electricity generated from each source to the total nonhydro electricity as weights for each nonhydro source. To investigate what motivates developing countries to diversify their nonhydro sources of renewable energy, we used a two-part model estimation. We proceed in two steps. First, we use a logit estimation to account for the decision on whether to adopt nonhydro sources or not. In the second step, conditional on the first step that developing countries will adopt nonhydro, we use ordinary least squares to investigate the determinants of diversification of nonhydro sources of renewable energy. We present the combined or average marginal effects from the two-part model estimation ${ }^{28}$. Results from Table 4.4, largely confirm the findings from the previous estimation. Since we now consider the share of electricity generated from each particular source, we observed a change in the magnitude of the estimates but the associated signs remain similar. The marginal effects can be interpreted as the usual OLS coefficient.

Higher income is significantly and positively associated with diversification but losses its significant with more added control variables. The nonlinear effect of income still holds suggesting that diversification non-monotonically increases with income. Population growth, oil price, renewable energy policies, technological innovations, financial development and human capital significantly and positively influence the diversification of nonhydro renewable energy. Conversely, abundance of hydropower decreases the likelihood that developing countries will diversify. Since hydropower is also a renewable energy, having it does not promote further the adoption of other sources of renewable energy. In line with the issue of carbon emissions, this shows that countries that already have renewable energy (i.e. hydropower) are likely to be less enthusiastic in adopting varied sources of renewable energy because they are already relatively low in carbon intensity. This confirms the findings of Popp et al. (2011) and Pfeiffer and Mulder (2013). Likewise, higher local oil production does not promote diversification of nonhydro sources of renewable energy. Abundance of oil decreases the attractiveness of venturing into renewable energy because it lowers concerns of energy security and will make renewable energy relatively expensive. Hence, developing countries endowed with fossil fuels are less likely to diversify sources of renewable energy. The majority of the factors that promote diversification such as technology, human resources, and access to finance are the major scarce inputs highlighted by Collier and Venables (2012) that hinder Africa in adopting renewable technologies.

FDI and ODA present similar results with our previous estimation. These external sources of funding do not contribute to the diversification of nonhydro sources of renewable energy in developing countries. However, the effect of energy imports on diversification is muted. Similarly Aquirre and Ibikunle (2014) and Popp et al. (2011) showed that energy import is not a main driver for renewable energy diffusion. Though our previous results finds significant effect of energy import on diversification, its effect is very marginal. Coastal

\footnotetext{
${ }^{28}$ Details of the two-part model estimation with the first stage (logit) and second stage (OLS) estimation can be found in the appendix.
} 
dummies showed robust influence on diversification suggesting that countries endowed with coastal areas are more likely to diversify their nonhydro sources of renewable energy.

Table 4.4. Average marginal effects from the two-parts model estimation (TPM).

\begin{tabular}{|c|c|c|c|c|c|c|}
\hline Variables & $\begin{array}{c}(8) \\
\text { TPM }\end{array}$ & $\begin{array}{c}(9) \\
\text { TPM }\end{array}$ & $\begin{array}{l}(10) \\
\text { TPM }\end{array}$ & $\begin{array}{l}(11) \\
\text { TPM }\end{array}$ & $\begin{array}{l}(12) \\
\text { TPM }\end{array}$ & $\begin{array}{l}(13) \\
\text { TPM }\end{array}$ \\
\hline $\log$ GDP pc & $\begin{array}{l}0.070 * * * \\
(0.0242)\end{array}$ & $\begin{array}{l}0.051 * * \\
(0.0242)\end{array}$ & $\begin{array}{l}0.023 \\
(0.0282)\end{array}$ & $\begin{array}{l}0.013 \\
(0.0288)\end{array}$ & $\begin{array}{l}-0.759 * * * \\
(0.2017)\end{array}$ & $\begin{array}{l}-0.736 * * * \\
(0.2025)\end{array}$ \\
\hline $\log$ GDP pc_sq & & & & & $\begin{array}{l}0.054 * * * \\
(0.0142)\end{array}$ & $\begin{array}{l}0.051 * * * \\
(0.0143)\end{array}$ \\
\hline energy import & $\begin{array}{l}-0.0001 \\
(0.0002)\end{array}$ & $\begin{array}{l}-0.00005 \\
(0.0002)\end{array}$ & $\begin{array}{l}0.0002 \\
(0.0003)\end{array}$ & $\begin{array}{l}0.0002 \\
(0.0003)\end{array}$ & $\begin{array}{l}0.00002 \\
(0.0003)\end{array}$ & $\begin{array}{l}0.0001 \\
(0.0003)\end{array}$ \\
\hline population growth & $\begin{array}{l}0.081 * * * \\
(0.0227)\end{array}$ & $\begin{array}{l}0.082 * * * \\
(0.0221)\end{array}$ & $\begin{array}{l}0.072 * * * \\
(0.0219)\end{array}$ & $\begin{array}{l}0.074 * * * \\
(0.0221)\end{array}$ & $\begin{array}{l}0.064 * * * \\
(0.0216)\end{array}$ & $\begin{array}{l}0.066 * * * \\
(0.0217)\end{array}$ \\
\hline oil price & $\begin{array}{l}0.010 * * * \\
(0.0022)\end{array}$ & $\begin{array}{l}0.008 * * * \\
(0.0022)\end{array}$ & $\begin{array}{l}0.009 * * * \\
(0.0024)\end{array}$ & $\begin{array}{l}0.008 * * * \\
(0.0024)\end{array}$ & $\begin{array}{l}0.008 * * * \\
(0.0024)\end{array}$ & $\begin{array}{l}0.007 * * * \\
(0.0024)\end{array}$ \\
\hline policy & & $\begin{array}{l}0.137 * * * \\
(0.0392)\end{array}$ & & $\begin{array}{l}0.059 \\
(0.0393)\end{array}$ & & $\begin{array}{l}0.046 \\
(0.0399)\end{array}$ \\
\hline log patents & $\begin{array}{l}0.038 * * * \\
(0.0043)\end{array}$ & $\begin{array}{l}0.033 * * * \\
(0.0045)\end{array}$ & $\begin{array}{l}0.023 * * * \\
(0.0048)\end{array}$ & $\begin{array}{l}0.021 * * * \\
(0.0049)\end{array}$ & $\begin{array}{l}0.020 * * * \\
(0.0048)\end{array}$ & $\begin{array}{l}0.019 * * * \\
(0.0050)\end{array}$ \\
\hline $\log$ finance dev & $\begin{array}{l}0.080 * * * \\
(0.0205)\end{array}$ & $\begin{array}{l}0.083 * * * \\
(0.0207)\end{array}$ & $\begin{array}{l}0.055^{* * * *} \\
(0.0201)\end{array}$ & $\begin{array}{l}0.058 * * * \\
(0.0202)\end{array}$ & $\begin{array}{l}0.059 * * * \\
(0.0192)\end{array}$ & $\begin{array}{l}0.061 * * * \\
(0.0193)\end{array}$ \\
\hline sec enrollment & $\begin{array}{l}-0.0001 \\
(0.0011)\end{array}$ & $\begin{array}{l}-0.00002 \\
(0.0011)\end{array}$ & $\begin{array}{l}0.005 * * * \\
(0.0011)\end{array}$ & $\begin{array}{l}0.005 * * * \\
(0.0011)\end{array}$ & $\begin{array}{l}0.005 * * * \\
(0.0011)\end{array}$ & $\begin{array}{l}0.005 * * * \\
(0.0011)\end{array}$ \\
\hline hydro energy & $\begin{array}{l}-0.087 * * * \\
(0.0195)\end{array}$ & $\begin{array}{l}-0.077 * * * \\
(0.0189)\end{array}$ & $\begin{array}{l}-0.283 * * * \\
(0.0263)\end{array}$ & $\begin{array}{l}-0.268 * * * \\
(0.0283)\end{array}$ & $\begin{array}{l}-0.297 * * * \\
(0.0264)\end{array}$ & $\begin{array}{l}-0.285 * * * \\
(0.0286)\end{array}$ \\
\hline oil prod'n & $\begin{array}{l}-0.013 * * * \\
(0.0026)\end{array}$ & $\begin{array}{l}-0.012 * * * \\
(0.0024)\end{array}$ & $\begin{array}{l}-0.008 * * * \\
(0.0031)\end{array}$ & $\begin{array}{l}-0.008 * * * \\
(0.0030)\end{array}$ & $\begin{array}{l}-0.010^{* * *} \\
(0.0031)\end{array}$ & $\begin{array}{l}-0.010 \text { *** } \\
(0.0030)\end{array}$ \\
\hline coal prod'n & $\begin{array}{l}-0.004 \\
(0.0107)\end{array}$ & $\begin{array}{l}0.001 \\
(0.011)\end{array}$ & $\begin{array}{l}0.010 \\
(0.0146)\end{array}$ & $\begin{array}{l}0.012 \\
(0.0141)\end{array}$ & $\begin{array}{l}0.015 \\
(0.0144)\end{array}$ & $\begin{array}{l}0.016 \\
(0.0140)\end{array}$ \\
\hline FDI & & & $\begin{array}{l}-0.014 * * \\
(0.0069)\end{array}$ & $\begin{array}{l}-0.014 * * \\
(0.0069)\end{array}$ & $\begin{array}{l}-0.016 * * \\
(0.0068)\end{array}$ & $\begin{array}{l}-0.016 * * \\
(0.0069)\end{array}$ \\
\hline ODA & & & $\begin{array}{l}-0.002 \\
(0.0027)\end{array}$ & $\begin{array}{l}-0.002 \\
(0.0027)\end{array}$ & $\begin{array}{l}-0.004 \\
(0.0027)\end{array}$ & $\begin{array}{l}-0.005^{*} \\
(0.0027)\end{array}$ \\
\hline coast & $\begin{array}{l}0.189 * * * \\
(0.0420)\end{array}$ & $\begin{array}{l}0.192 * * * \\
(0.0417)\end{array}$ & $\begin{array}{l}0.328 * * * \\
(0.0486)\end{array}$ & $\begin{array}{l}0.317 * * * \\
(0.0487)\end{array}$ & $\begin{array}{l}0.297 * * * \\
(0.0478)\end{array}$ & $\begin{array}{l}0.290 * * * \\
(0.0479)\end{array}$ \\
\hline $\begin{array}{l}\text { regional dummies } \\
\text { year dummies }\end{array}$ & $\begin{array}{l}\text { yes } \\
\text { yes }\end{array}$ & $\begin{array}{l}\text { yes } \\
\text { yes }\end{array}$ & $\begin{array}{l}\text { yes } \\
\text { yes }\end{array}$ & $\begin{array}{l}\text { yes } \\
\text { yes }\end{array}$ & $\begin{array}{l}\text { yes } \\
\text { yes }\end{array}$ & $\begin{array}{l}\text { yes } \\
\text { yes }\end{array}$ \\
\hline observations & 1,386 & 1,386 & 1,232 & 1,232 & 1,232 & 1,232 \\
\hline
\end{tabular}

Note: Our dependent variable is the computed diversity index or the weighted number of nonhydro sources of renewable energy. We use the share electricity generated from each particular source as weights. Robust standard errors in parentheses, $* * * \mathrm{p}<0.01, * * \mathrm{p}<0.05, * \mathrm{p}<0.1$ 


\subsubsection{Does Kyoto protocol matters?}

The adoption of Kyoto protocol in late 1997, marks a significant shift in global climate policy. We therefore, investigate its effect on the diversification of nonhydro renewable energy by including a time dummy from 1998 onward.. Figure 4.4 shows a strong Kyoto protocol effect in which we observe a substantial increase in the number of nonhydro renewable energy sources adopted by developing countries. We incorporate this potential impact of Kyoto protocol on nonhydro renewable energy diversification in developing countries by including a time dummy from 1998 onwards $^{29}$. Although Kyoto protocol did not place a heavy burden on developing countries to reduce emissions, results in Table 4.5 consistently show a strong positive and significant effect of Kyoto protocol on the diversification of nonhydro sources of renewable energy. This suggests that greater environmental awareness has led to a greater diffusion of renewable energy in developing countries. These results align well with the previous findings in the literature (Brunnschweiler 2010; Johnstone et al. 2010; Pfeiffer and Mulder 2013; Aguirre and Ibikunle 2014). Brunnschweiler (2010) found that most of the increase in renewable energy did not just come from hydropower but also from a 27 -fold increase in the electricity generated from nonhydro sources after the adoption of Kyoto protocol. Our results add to this strand of literature by presenting evidence that Kyoto protocol also significantly influences the diversification of nonhydro sources of renewable energy in developing countries.

Focusing on the coefficients of Kyoto protocol in Table 4.5, we observed a difference in the magnitudes of the estimates between the negative binomial and two-part model. The difference can be explained by the type of data used in the estimation. For the negative binomial, our dependent variable is a count data capturing the number of nonhydro sources of renewal energy adopted by each country while for the two-part model, we use the diversity index or the weighted number of nonhydro sources. Estimates from the negative binomial show that after the adoption of Kyoto protocol, the log of expected count of nonhydro sources of renewable energy adopted by developing countries increases by $72 \%$ while estimates from TPM suggests that the index of diversification increases by $18 \%$ since the adoption of Kyoto protocol. Though the magnitudes differ, the associated relationship is consistent across several specifications. Since we employ different estimation techniques, we are keener in emphasizing the associated relationship rather than expounding on the coefficients. This implies that a wider diversification of nonhydro sources of renewable energy took place after the adoption of Kyoto Protocol in the late $1997^{30}$. The robust effect of Kyoto protocol on the diversification can be strongly linked to the growing number of clean development mechanism (CDM) adopted in developing countries. The CDM as defined under Kyoto protocol allows an industrialized country with a commitment to reduce emissions to implement an emission reduction project in developing countries (UNFCCC 2014). CDM carries a strong financial incentive for developing countries to adopt renewable energy.

\footnotetext{
${ }^{29}$ We drop our time dummies when we introduce Kyoto Protocol in the specification to avoid collinearity since Kyoto protocol also captures time effects.

${ }^{30}$ This is further supported by the results using time dummies (Appendix Table 10). Results show that the number of nonhydro sources adopted by developing countries was lower before 1998 but was higher after 1998 suggesting that the effect of Kyoto Protocol on diversification is plausible.
} 
Looking at the control variables, results show that most of the associated relationships are consistent with our previous findings.

Table 4.5. Effect of Kyoto Protocol on the diversification of nonhydro sources.

\begin{tabular}{|c|c|c|c|c|c|c|c|c|}
\hline VARIABLES & $\begin{array}{c}(14) \\
\text { Negbin }\end{array}$ & $\begin{array}{c}(15) \\
\text { Negbin }\end{array}$ & $\begin{array}{c}(16) \\
\text { Negbin }\end{array}$ & $\begin{array}{c}(17) \\
\text { Negbin }\end{array}$ & $\begin{array}{l}(18) \\
\text { TPM }\end{array}$ & $\begin{array}{l}\text { (19) } \\
\text { TPM }\end{array}$ & $\begin{array}{l}\text { (20) } \\
\text { TPM }\end{array}$ & $\begin{array}{l}(21) \\
\text { TPM }\end{array}$ \\
\hline $\log$ GDP pc & $\begin{array}{l}0.229^{* * * *} \\
(0.0668)\end{array}$ & $\begin{array}{l}0.103^{*} \\
(0.0616)\end{array}$ & $\begin{array}{c}-1.948^{* * *} \\
(0.4883)\end{array}$ & $\begin{array}{c}-1.796^{* * *} \\
(0.4805)\end{array}$ & $\begin{array}{l}0.064^{* * *} \\
(0.0244)\end{array}$ & $\begin{array}{l}0.043^{*} \\
(0.0243)\end{array}$ & $\begin{array}{l}-0.796^{* * *} \\
(0.2087)\end{array}$ & $\begin{array}{l}-0.771^{* * *} \\
(0.2095)\end{array}$ \\
\hline $\log$ GDP pc_sq & & & $\begin{array}{l}0.130^{* * *} \\
(0.0316)\end{array}$ & $\begin{array}{l}0.116^{* * *} \\
(0.0309)\end{array}$ & & & $0.056^{* * *}$ & $\begin{array}{l}0.054^{* * *} \\
(0.0148)\end{array}$ \\
\hline energy import & $\begin{array}{l}0.001^{* *} \\
(0.0004)\end{array}$ & $\begin{array}{l}0.002^{* * *} \\
(0.0005)\end{array}$ & $\begin{array}{l}0.001^{* *} \\
(0.0006)\end{array}$ & $\begin{array}{l}0.002^{* * * *} \\
(0.0006)\end{array}$ & $\begin{array}{l}-0.0001 \\
(0.0002)\end{array}$ & $\begin{array}{l}-0.00002 \\
(0.0002)\end{array}$ & $\begin{array}{l}-0.00002 \\
(0.0003)\end{array}$ & $\begin{array}{l}0.00003 \\
(0.0003)\end{array}$ \\
\hline population growth & $\begin{array}{l}-0.015 \\
(0.0521)\end{array}$ & $\begin{array}{c}0.045 \\
(0.0470)\end{array}$ & $\begin{array}{c}0.082 \\
(0.0551)\end{array}$ & $\begin{array}{l}0.120^{* * *} \\
(0.0510)\end{array}$ & $\begin{array}{l}0.073^{* * * *} \\
(0.0232)\end{array}$ & $\begin{array}{l}0.075^{* * *} \\
(0.0224)\end{array}$ & $\begin{array}{l}0.052^{* * *} \\
(0.0231)\end{array}$ & $\begin{array}{l}0.054^{* *} \\
(0.0229)\end{array}$ \\
\hline oil price & $\begin{array}{l}0.003^{* *} \\
(0.0011)\end{array}$ & $\begin{array}{l}0.0004 \\
(0.0011)\end{array}$ & $\begin{array}{l}0.003^{* * *} \\
(0.0011)\end{array}$ & $\begin{array}{c}0.001 \\
(0.0011)\end{array}$ & $\begin{array}{l}0.002^{* * * *} \\
(0.0006)\end{array}$ & $\begin{array}{l}0.001 \\
(0.0007)\end{array}$ & $\begin{array}{l}0.001^{* *} \\
(0.0006)\end{array}$ & $\begin{array}{l}0.001^{*} \\
(0.0007)\end{array}$ \\
\hline $\log$ finance dev & $\begin{array}{l}0.144^{* * * *} \\
(0.0435)\end{array}$ & $\begin{array}{l}0.168^{* * * *} \\
(0.0413)\end{array}$ & $\begin{array}{l}0.120^{* * *} \\
(0.0511)\end{array}$ & $\begin{array}{l}0.145^{* * * *} \\
(0.0504)\end{array}$ & & $\begin{array}{l}0.151^{\text {**** }} \\
(0.0386)\end{array}$ & & $\begin{array}{l}0.059 \\
(0.0399)\end{array}$ \\
\hline policy & & $\begin{array}{l}0.589^{* * * *} \\
(0.0724)\end{array}$ & & $\begin{array}{l}0.344^{* * * *} \\
(0.0746)\end{array}$ & $\begin{array}{l}0.038^{* * * *} \\
(0.0043)\end{array}$ & $\begin{array}{l}0.033^{* * * *} \\
(0.0045)\end{array}$ & $\begin{array}{l}0.022^{* * * *} \\
(0.0047)\end{array}$ & $\begin{array}{l}0.020^{* * *} \\
(0.0049)\end{array}$ \\
\hline log patents & & & $\begin{array}{l}0.081^{* * * *} \\
(0.0129)\end{array}$ & $\begin{array}{l}0.064^{* * *} \\
(0.0128)\end{array}$ & $\begin{array}{l}0.077^{* * * *} \\
(0.0197)\end{array}$ & $\begin{array}{l}0.081^{* * *} \\
(0.0198)\end{array}$ & $\begin{array}{l}0.059^{* * *} \\
(0.0185)\end{array}$ & $\begin{array}{l}0.061^{* * * *} \\
(0.0184)\end{array}$ \\
\hline sec enrollment & $\begin{array}{l}0.007^{* *} \\
(0.0027)\end{array}$ & $\begin{array}{l}0.006^{* *} \\
(0.0026)\end{array}$ & $\begin{array}{c}0.016^{* * *} \\
(0.0026)\end{array}$ & $\begin{array}{l}0.016^{* * *} \\
(0.0025)\end{array}$ & $\begin{array}{l}0.001 \\
(0.0010)\end{array}$ & $\begin{array}{l}0.001 \\
(0.0010)\end{array}$ & $\begin{array}{l}0.006^{* * *} \\
(0.0010)\end{array}$ & $\begin{array}{l}0.006^{* * * *} \\
(0.0010)\end{array}$ \\
\hline Kyoto protocol & $\begin{array}{c}0.609^{* * * *} \\
(0.0814)\end{array}$ & $\begin{array}{c}0.523^{* * * *} \\
(0.0818)\end{array}$ & $\begin{array}{l}0.598^{* * * *} \\
(0.0840)\end{array}$ & $\begin{array}{c}0.544^{* * * *} \\
(0.0859)\end{array}$ & $\begin{array}{l}0.252^{* * *} \\
(0.0354)\end{array}$ & $\begin{array}{l}0.228^{* * * *} \\
(0.0350)\end{array}$ & $\begin{array}{l}0.193^{* * *} \\
(0.0356)\end{array}$ & $\begin{array}{l}0.184^{* * *} \\
(0.0362)\end{array}$ \\
\hline hydro energy & $\begin{array}{c}-0.423^{* * * *} \\
(0.0849)\end{array}$ & $\begin{array}{c}-0.307^{* * * *} \\
(0.0802)\end{array}$ & $\begin{array}{c}-0.869^{* * * * *} \\
(0.0755)\end{array}$ & $\begin{array}{c}-0.803^{* * * *} \\
(0.0734)\end{array}$ & $\begin{array}{l}-0.078^{* * * *} \\
(0.0206)\end{array}$ & $\begin{array}{l}-0.067^{* * * *} \\
(0.0200)\end{array}$ & $\begin{array}{l}-0.294^{* * * *} \\
(0.0267)\end{array}$ & $\begin{array}{l}-0.279^{* * * *} \\
(0.0288)\end{array}$ \\
\hline oil prod'n & $\begin{array}{c}-0.014^{* * *} \\
(0.0051)\end{array}$ & $\begin{array}{l}-0.008 \\
(0.0049)\end{array}$ & $\begin{array}{c}-0.030^{* * * *} \\
(0.0099)\end{array}$ & $\begin{array}{c}-0.025^{* * *} \\
(0.0094)\end{array}$ & $\begin{array}{l}-0.012^{* * *} \\
(0.0027)\end{array}$ & $\begin{array}{l}-0.011^{* * * *} \\
(0.0026)\end{array}$ & $\begin{array}{l}-0.011^{* * *} \\
(0.0032)\end{array}$ & $\begin{array}{l}-0.010^{* * *} \\
(0.0031)\end{array}$ \\
\hline coal prod'n & $\begin{array}{l}0.085^{* * *} \\
(0.0200)\end{array}$ & $\begin{array}{l}0.111^{* * *} \\
(0.0224)\end{array}$ & $\begin{array}{l}0.080^{* *} \\
(0.0346)\end{array}$ & $\begin{array}{l}0.102^{* * *} \\
(0.0355)\end{array}$ & $\begin{array}{l}-0.006 \\
(0.0107)\end{array}$ & $\begin{array}{l}-0.001 \\
(0.0107)\end{array}$ & $\begin{array}{l}0.012 \\
(0.0145)\end{array}$ & $\begin{array}{l}0.013 \\
(0.0140)\end{array}$ \\
\hline FDI & & & $\begin{array}{l}-0.038^{* *} \\
(0.0182)\end{array}$ & $\begin{array}{c}-0.038^{* *} \\
(0.0170)\end{array}$ & & & $\begin{array}{l}-0.015^{* *} \\
(0.0062)\end{array}$ & $\begin{array}{l}-0.015^{* *} \\
(0.0061)\end{array}$ \\
\hline ODA & & & $\begin{array}{l}-0.020 \\
(0.0124)\end{array}$ & $\begin{array}{l}-0.021^{*} \\
(0.0121)\end{array}$ & & & $\begin{array}{l}-0.002 \\
(0.0027)\end{array}$ & $\begin{array}{l}-0.003 \\
(0.0027)\end{array}$ \\
\hline coast & $\begin{array}{l}0.926^{* * *} \\
(0.1334)\end{array}$ & $\begin{array}{l}0.899^{* * *} \\
(0.1342)\end{array}$ & $\begin{array}{l}0.961^{* * *} \\
(0.1468)\end{array}$ & $\begin{array}{l}0.942^{* * * *} \\
(0.1463)\end{array}$ & $\begin{array}{l}0.19^{7 * * *} \\
(0.0415)\end{array}$ & $\begin{array}{l}0.201^{\text {*** }} \\
(0.0413)\end{array}$ & $\begin{array}{l}0.303^{* * *} \\
(0.0472)\end{array}$ & $\begin{array}{l}0.295^{* * *} \\
(0.0473)\end{array}$ \\
\hline regional dummies & yes & yes & yes & yes & yes & yes & yes & yes \\
\hline year dummies & no & no & no & no & no & no & no & no \\
\hline constant & $\begin{array}{c}-4.674^{* * *} \\
(0.4372)\end{array}$ & $\begin{array}{c}-3.783^{* * *} \\
(0.4247)\end{array}$ & $\begin{array}{c}3.658^{*} \\
(1.9365)\end{array}$ & $\begin{array}{c}3.272^{*} \\
(1.9064)\end{array}$ & & & & \\
\hline ervations & 1386 & 1386 & 1232 & 1232 & & $8 f$ & 232 & 232 \\
\hline
\end{tabular}

Note: The dependent variable when using negative binomial regression is the number of nonhydro sources of renewable energy while the dependent variable when using TPM regression is the derived diversity index or simply the weighted number of nonhydro sources. Robust standard errors in parentheses, $* * * \mathrm{p}<0.01, * * \mathrm{p}<0.05$, * $\mathrm{p}<0.1$ 


\subsubsection{Extended results and robustness check}

Based on Figure 4.6 (taken from Pfeiffer and Mulder's (2013) paper) the aggregate production of nonhydro renewable energy of large developing countries like Brazil, Russia, India, China and South Africa (BRICS) behaves similarly with the aggregate production of nonhydro renewable energy for all developing countries. Thus, it is likely that these large developing countries drive the results from our previous estimation. To control for this potential bias, we conduct an extended estimation using negative binomial regression excluding the BRICS.

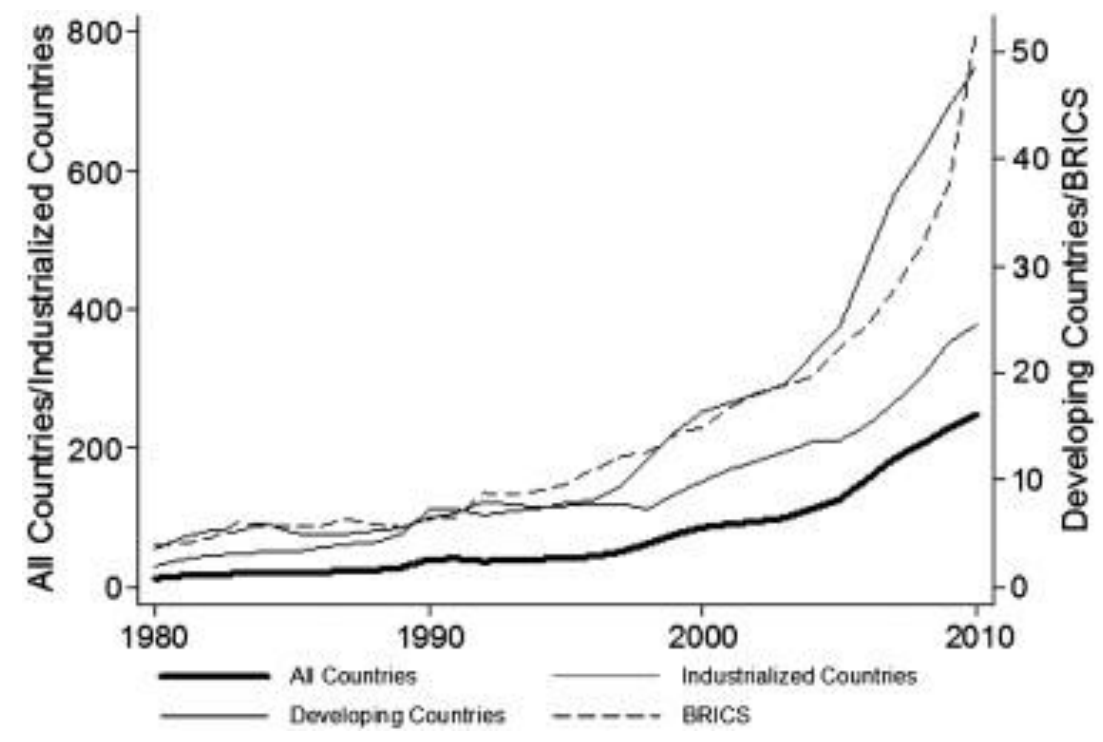

Source: Pfeiffer and Mulder (2013) with data based on IEA.

Figure 4.6. Nonhydro renewable energy per capita production (measured in kWh).

In general, the results of the estimation excluding BRICS are consistent with our previous findings. It appears that our results are very robust even with the exclusion of BRICS. Income posted a positive association with diversification but again losses its significance with the addition of a policy variable. However, what we would like to emphasize is the significant nonlinear effect of income on diversification. The nonlinear effect is consistent and is robust in all specifications even with the exclusion of BRICS. This further supports our claim that there exists a certain U-shaped type of relationship between income and diversification.

With the exclusion of BRICS, results from Table 4.6 strengthens our argument that the diversification of nonhydro sources of renewable energy is facilitated with increasing energy imports, higher population growth, higher crude oil prices, polices promoting renewable energy, advances in technology, access to finance and improvement in human resources. In contrast, abundance of hydro power, local oil production, FDI and ODA delays diversification. In addition, Kyoto protocol and coastal dummies have a strong influence on diversification. 
Table 4.6. Negative binomial regression excluding BRICS.

\begin{tabular}{|c|c|c|c|c|c|c|c|c|}
\hline Variables & $\begin{array}{c}(22) \\
\text { Negbin } \\
\end{array}$ & $\begin{array}{c}(23) \\
\text { Negbin } \\
\end{array}$ & $\begin{array}{c}(24) \\
\text { Negbin } \\
\end{array}$ & $\begin{array}{c}(25) \\
\text { Negbin } \\
\end{array}$ & $\begin{array}{c}(26) \\
\text { Negbin } \\
\end{array}$ & $\begin{array}{c}(27) \\
\text { Negbin } \\
\end{array}$ & $\begin{array}{c}(28) \\
\text { Negbin } \\
\end{array}$ & $\begin{array}{c}(29) \\
\text { Negbin } \\
\end{array}$ \\
\hline $\log$ GDP pc & $\begin{array}{l}0.24^{* * *} \\
(0.070)\end{array}$ & $\begin{array}{c}0.07 \\
(0.065)\end{array}$ & $\begin{array}{l}-3.19^{* * *} \\
(0.541)\end{array}$ & $\begin{array}{l}-3.01^{\text {**** }} \\
(0.529)\end{array}$ & $\begin{array}{l}0.24^{* * *} \\
(0.071)\end{array}$ & $\begin{array}{c}0.06 \\
(0.066)\end{array}$ & $\begin{array}{l}-3.34^{* * *} \\
(0.545)\end{array}$ & $\begin{array}{c}-3.15^{* * *} \\
(0.534)\end{array}$ \\
\hline $\log$ GDP pc_sq & & & $\begin{array}{l}0.21^{* * *} \\
(0.035)\end{array}$ & $\begin{array}{l}0.19^{* * *} \\
(0.034)\end{array}$ & & & $\begin{array}{l}0.22^{* * *} \\
(0.035)\end{array}$ & $\begin{array}{l}0.20^{* * *} \\
(0.034)\end{array}$ \\
\hline energy import & $\begin{array}{c}0.0005 \\
(0.0003)\end{array}$ & $\begin{array}{l}0.002^{* * *} \\
(0.0005)\end{array}$ & $\begin{array}{l}0.001^{*} \\
(0.001)\end{array}$ & $\begin{array}{c}0.002^{* * *} \\
(0.001)\end{array}$ & $\begin{array}{c}0.0005 \\
(0.0003)\end{array}$ & $\begin{array}{l}0.002^{* * *} \\
(0.0005)\end{array}$ & $\begin{array}{c}0.001 \\
(0.001)\end{array}$ & $\begin{array}{c}0.002^{* * *} \\
(0.001)\end{array}$ \\
\hline population growth & $\begin{array}{c}0.07 \\
(0.048)\end{array}$ & $\begin{array}{l}0.12^{* * *} \\
(0.040)\end{array}$ & $\begin{array}{l}0.11^{* *} \\
(0.050)\end{array}$ & $\begin{array}{l}0.15^{* * *} \\
(0.045)\end{array}$ & $\begin{array}{c}0.06 \\
(0.050)\end{array}$ & $\begin{array}{l}0.11^{* *} \\
(0.043)\end{array}$ & $\begin{array}{c}0.08 \\
(0.055)\end{array}$ & $\begin{array}{l}0.12^{* *} \\
(0.051)\end{array}$ \\
\hline oil price & $\begin{array}{l}0.02^{* * * *} \\
(0.004)\end{array}$ & $\begin{array}{l}0.02^{* * *} \\
(0.004)\end{array}$ & $\begin{array}{l}0.02^{* * * *} \\
(0.004)\end{array}$ & $\begin{array}{l}0.02^{* * * *} \\
(0.004)\end{array}$ & $\begin{array}{c}0.004^{* * *} \\
(0.001)\end{array}$ & $\begin{array}{l}0.002^{*} \\
(0.001)\end{array}$ & $\begin{array}{l}0.003^{* *} \\
(0.001)\end{array}$ & $\begin{array}{c}0.001 \\
(0.001)\end{array}$ \\
\hline policy & & $\begin{array}{l}0.66^{* * *} \\
(0.075)\end{array}$ & & $\begin{array}{l}0.40^{* * *} \\
(0.076)\end{array}$ & & $\begin{array}{l}0.69^{* * *} \\
(0.077)\end{array}$ & & $\begin{array}{l}0.42^{* * *} \\
(0.078)\end{array}$ \\
\hline $\log$ patents & & & $\begin{array}{l}0.05^{* * *} \\
(0.015)\end{array}$ & $\begin{array}{l}0.03^{* *} \\
(0.014)\end{array}$ & & & $\begin{array}{l}0.05^{* * *} \\
(0.015)\end{array}$ & $\begin{array}{l}0.03^{* *} \\
(0.014)\end{array}$ \\
\hline $\log$ finance dev & $\begin{array}{l}0.11^{* *} \\
(0.047)\end{array}$ & $\begin{array}{l}0.15^{* * *} \\
(0.043)\end{array}$ & $\begin{array}{l}0.16^{* * * *} \\
(0.050)\end{array}$ & $\begin{array}{l}0.19^{* * *} \\
(0.049)\end{array}$ & $\begin{array}{c}0.09^{*} \\
(0.047)\end{array}$ & $\begin{array}{l}0.14^{* * *} \\
(0.043)\end{array}$ & $\begin{array}{l}0.15^{* * *} \\
(0.050)\end{array}$ & $\begin{array}{l}0.18^{* * *} \\
(0.049)\end{array}$ \\
\hline sec enrollment & $\begin{array}{c}0.003 \\
(0.003)\end{array}$ & $\begin{array}{l}0.001 \\
(0.003)\end{array}$ & $\begin{array}{l}0.01^{* * *} \\
(0.003)\end{array}$ & $\begin{array}{l}0.01^{* * *} \\
(0.003)\end{array}$ & $\begin{array}{l}0.01^{* *} \\
(0.003)\end{array}$ & $\begin{array}{c}0.003 \\
(0.003)\end{array}$ & $\begin{array}{l}0.02^{* * *} \\
(0.003)\end{array}$ & $\begin{array}{l}0.02^{* * *} \\
(0.003)\end{array}$ \\
\hline Kyoto protocol & & & & & $\begin{array}{l}0.64^{* * * *} \\
(0.083)\end{array}$ & $\begin{array}{l}0.57^{* * *} \\
(0.083)\end{array}$ & $\begin{array}{l}0.59^{* * *} \\
(0.090)\end{array}$ & $\begin{array}{l}0.54^{* * *} \\
(0.091)\end{array}$ \\
\hline hydro energy & $\begin{array}{c}-0.45^{* * *} \\
(0.090)\end{array}$ & $\begin{array}{l}-0.32^{* * *} \\
(0.079)\end{array}$ & $\begin{array}{c}-0.88^{* * *} \\
(0.074)\end{array}$ & $\begin{array}{c}-0.79^{* * *} \\
(0.070)\end{array}$ & $\begin{array}{c}-0.45^{* * *} \\
(0.092)\end{array}$ & $\begin{array}{c}-0.31^{* * *} \\
(0.082)\end{array}$ & $\begin{array}{l}-0.90^{* * *} \\
(0.078)\end{array}$ & $\begin{array}{c}-0.79^{* * *} \\
(0.073)\end{array}$ \\
\hline oil prod'n & $\begin{array}{l}-0.02^{* * *} \\
(0.006)\end{array}$ & $\begin{array}{l}-0.02^{* * *} \\
(0.006)\end{array}$ & $\begin{array}{l}-0.03^{* * *} \\
(0.011)\end{array}$ & $\begin{array}{l}-0.02^{* *} \\
(0.010)\end{array}$ & $\begin{array}{l}-0.02^{* * *} \\
(0.006)\end{array}$ & $\begin{array}{l}-0.02^{* * *} \\
(0.006)\end{array}$ & $\begin{array}{l}-0.03^{* * *} \\
(0.010)\end{array}$ & $\begin{array}{l}-0.03^{\text {**** }} \\
(0.009)\end{array}$ \\
\hline coal prod'n & $\begin{array}{c}0.03 \\
(0.022)\end{array}$ & $\begin{array}{l}0.05^{* *} \\
(0.025)\end{array}$ & $\begin{array}{l}0.12^{* *} \\
(0.050)\end{array}$ & $\begin{array}{l}0.13^{* *} \\
(0.050)\end{array}$ & $\begin{array}{c}0.03 \\
(0.022)\end{array}$ & $\begin{array}{l}0.05^{* *} \\
(0.024)\end{array}$ & $\begin{array}{l}0.12^{* *} \\
(0.047)\end{array}$ & $\begin{array}{l}0.12^{* * *} \\
(0.047)\end{array}$ \\
\hline FDI & & & $\begin{array}{l}-0.06^{* * *} \\
(0.023)\end{array}$ & $\begin{array}{l}-0.06^{* * *} \\
(0.022)\end{array}$ & & & $\begin{array}{l}-0.06^{* * *} \\
(0.019)\end{array}$ & $\begin{array}{l}-0.05^{* * *} \\
(0.018)\end{array}$ \\
\hline ODA & & & $\begin{array}{l}-0.03^{* *} \\
(0.012)\end{array}$ & $\begin{array}{l}-0.03^{* *} \\
(0.012)\end{array}$ & & & $\begin{array}{l}-0.03^{* *} \\
(0.013)\end{array}$ & $\begin{array}{l}-0.03^{* *} \\
(0.012)\end{array}$ \\
\hline coast & $\begin{array}{l}0.72^{* * *} \\
(0.139)\end{array}$ & $\begin{array}{l}0.71^{* * *} \\
(0.136)\end{array}$ & $\begin{array}{l}0.90^{* * *} \\
(0.152)\end{array}$ & $\begin{array}{l}0.87^{* * *} \\
(0.151)\end{array}$ & $\begin{array}{l}0.76^{* * *} \\
(0.137)\end{array}$ & $\begin{array}{l}0.73^{* * *} \\
(0.134)\end{array}$ & $\begin{array}{l}0.92^{* * *} \\
(0.149)\end{array}$ & $\begin{array}{l}0.88^{* * *} \\
(0.149)\end{array}$ \\
\hline $\begin{array}{l}\text { regional dummies } \\
\text { year dummies }\end{array}$ & $\begin{array}{l}\text { yes } \\
\text { yes }\end{array}$ & $\begin{array}{l}\text { yes } \\
\text { yes }\end{array}$ & $\begin{array}{l}\text { yes } \\
\text { yes }\end{array}$ & $\begin{array}{l}\text { yes } \\
\text { yes }\end{array}$ & $\begin{array}{l}\text { yes } \\
\text { no }\end{array}$ & $\begin{array}{c}\text { yes } \\
\text { no }\end{array}$ & $\begin{array}{c}\text { yes } \\
\text { no }\end{array}$ & $\begin{array}{l}\text { yes } \\
\text { no }\end{array}$ \\
\hline constant & $\begin{array}{l}-5.48^{* * *} \\
(0.540)\end{array}$ & $\begin{array}{l}-4.16^{* * *} \\
(0.524)\end{array}$ & $\begin{array}{l}7.37^{* * *} \\
(2.184)\end{array}$ & $\begin{array}{l}7.10^{* * *} \\
(2.144)\end{array}$ & $\begin{array}{l}-4.51^{* * *} \\
(0.466)\end{array}$ & $\begin{array}{l}-3.22^{* * *} \\
(0.454)\end{array}$ & $\begin{array}{l}8.95^{* * *} \\
(2.173)\end{array}$ & $\begin{array}{l}8.61^{* * *} \\
(2.137)\end{array}$ \\
\hline observations & 1,300 & 1,300 & 1,164 & 1,164 & 1,300 & 1,300 & 1,164 & 1,164 \\
\hline
\end{tabular}

Note: The dependent variable is the number of nonhydro sources of renewable energy adopted by developing countries.

Robust standard errors in parentheses, $* * * p<0.01$, ** $\mathrm{p}<0.05, * \mathrm{p}<0.1$ 
For the robustness check, we run the same specification using our measure of diversification and include all samples in the analysis. We use another popular method in dealing with zero observations since we have a substantial number of countries who have not invested in any nonhydro sources of renewable energy. For this case, we use the Poisson pseudo-maximum likelihood (PPML) estimation technique. Table 4.7 presents the estimation results using PPML. Results of PPML largely confirm our previous findings. Contrary to our previous results, income does not entirely loses its significance with added regressors. Its effect is robust and consistent across specifications. Higher income is associated with diversification and in addition, the nonlinear effect of income still holds. This reaffirms our observation that a U-shaped kind of relationship exists between income and diversification with a turning point around US\$2000, falling just within range of the current average per capita income. In addition, we also document a positive relationship between energy import and diversification confirming our previous findings that higher dependence on external sources of energy positively contributes to the diversification of nonhydro sources of renewable energy. Growing population is coupled with increasing demand for energy thus, we observe that higher population growth rates are positively associated with diversification. Energy generated from nonhydro sources will potentially fill this increasing demand for energy. Similarly, higher crude oil price is associated with increasing diversification. Developing countries are vulnerable to increases in world market oil price hence diversifying sources of renewable energy can potentially shield them from these external price shocks.

The diversification of nonhydro sources of renewable energy is further supported by technological innovation, financial development, implementation of polices promoting renewable energy, and improvement in human capital. Estimates are highly significant across several specifications except for secondary enrollment where in cases some coefficients are not significant. This confirms the argument presented by Collier and Venables (2012) that technology, skill, market development and sound governance promotes the adoption of renewable energy. In contrast, oil production and abundance of hydropower is negatively associated with diversification. As with our previous results, local abundance of coal is positively associated with diversification. Perhaps a cautious explanation maybe that coal production complements nonhydro renewable energy but this merits in-depth investigation, which we do not address in this current paper. Since the adoption of Kyoto protocol, there has been a wider diversification of nonhydro sources and developing countries endowed with coastal areas are more likely to diversify nonhydro sources of renewable energy. And again, there is no evidence showing that FDI and ODA support diversification.

For a further robustness check, we use the electricity generated from each source measured in thousand kilowatt-hours $(\mathrm{kWH})$ as dependent variables and check whether our results still hold. We sum the electricity generated from solar, wind, geothermal, waste and biomass and use electricity per capita as our independent variable. By using the electricity per capita generated from nonhydro sources, we are muting the concept of diversification and instead emphasize the total generation of renewable energy regardless of its source. We refrain from using the share of renewal energy to total energy production to avoid possible correlations between total energy productions with oil and coal production. 
Table 4.7. Robustness check using PPML with diversity index as a dependent variable.

\begin{tabular}{|c|c|c|c|c|c|c|c|c|}
\hline VARIABLES & $\begin{array}{c}(30) \\
\text { PPML } \\
\end{array}$ & $\begin{array}{c}(31) \\
\text { PPML } \\
\end{array}$ & $\begin{array}{c}(32) \\
\text { PPML } \\
\end{array}$ & $\begin{array}{c}(33) \\
\text { PPML } \\
\end{array}$ & $\begin{array}{c}(34) \\
\text { PPML } \\
\end{array}$ & $\begin{array}{c}(35) \\
\text { PPML } \\
\end{array}$ & $\begin{array}{c}(36) \\
\text { PPML } \\
\end{array}$ & $\begin{array}{c}(37) \\
\text { PPML } \\
\end{array}$ \\
\hline \multirow[t]{2}{*}{$\log$ GDP pc } & $0.14^{* *}$ & $0.11^{*}$ & $-1.59^{* * *}$ & $-1.50^{* * *}$ & $0.13^{* *}$ & $0.10^{*}$ & $-1.60^{* * *}$ & $-1.51^{* * *}$ \\
\hline & $(0.055)$ & $(0.057)$ & $(0.481)$ & $(0.477)$ & $(0.057)$ & $(0.058)$ & $(0.491)$ & (0.489) \\
\hline \multirow[t]{2}{*}{$\log$ GDP pc_sq } & & & $0.10^{* * *}$ & $0.10^{* * *}$ & & & $0.11^{* * *}$ & $0.10^{* * *}$ \\
\hline & & & $(0.031)$ & $(0.031)$ & & & $(0.032)$ & $(0.032)$ \\
\hline \multirow[t]{2}{*}{ energy import } & $0.001^{* *}$ & $0.001^{* *}$ & $0.001^{*}$ & $0.001^{* *}$ & $0.001^{* *}$ & $0.001^{* *}$ & $0.001^{*}$ & $0.001^{* *}$ \\
\hline & $(0.0004)$ & $(0.0004)$ & $(0.001)$ & $(0.001)$ & $(0.0004)$ & $(0.0004)$ & $(0.001)$ & $(0.001)$ \\
\hline \multirow[t]{2}{*}{ population growth } & $0.16^{* * *}$ & $0.16^{* * *}$ & $0.18^{* * * *}$ & $0.19^{* * *}$ & $0.15^{* * * *}$ & $0.15^{* * *}$ & $0.16^{* * *}$ & $0.17^{* * *}$ \\
\hline & $(0.041)$ & $(0.039)$ & $(0.043)$ & $(0.043)$ & $(0.044)$ & $(0.042)$ & $(0.049)$ & $(0.048)$ \\
\hline \multirow[t]{2}{*}{ oil price } & $0.02^{* * *}$ & $0.01^{* * * *}$ & $0.01^{* * * *}$ & $0.01^{* * * *}$ & $0.003^{* * *}$ & 0.002 & 0.001 & 0.001 \\
\hline & $(0.003)$ & $(0.003)$ & $(0.004)$ & $(0.004)$ & $(0.001)$ & $(0.001)$ & $(0.001)$ & $(0.001)$ \\
\hline \multirow[t]{2}{*}{ policy } & & $0.19^{* * *}$ & & $0.13^{*}$ & & $0.21^{* * *}$ & & $0.13^{*}$ \\
\hline & & $(0.068)$ & & $(0.072)$ & & $(0.068)$ & & $(0.073)$ \\
\hline \multirow[t]{2}{*}{ log patents } & $0.08^{* * *}$ & $0.07^{* * *}$ & $0.04^{* * *}$ & $0.04^{* * *}$ & $0.08^{* * *}$ & $0.07^{* * *}$ & $0.05^{* * *}$ & $0.04^{* * *}$ \\
\hline & $(0.011)$ & $(0.011)$ & $(0.013)$ & $(0.013)$ & $(0.011)$ & $(0.011)$ & $(0.013)$ & $(0.013)$ \\
\hline \multirow[t]{2}{*}{$\log$ finance dev } & $0.17^{* * *}$ & $0.18^{* * *}$ & $0.20^{* * *}$ & $0.21^{* * *}$ & $0.16^{* * *}$ & $0.16^{* * *}$ & $0.17^{* * *}$ & $0.18^{* * *}$ \\
\hline & $(0.047)$ & $(0.048)$ & $(0.055)$ & $(0.056)$ & $(0.047)$ & $(0.047)$ & $(0.053)$ & $(0.053)$ \\
\hline \multirow[t]{2}{*}{ sec enrollment } & -0.0002 & -0.00002 & $0.01^{* * *}$ & $0.01^{* * *}$ & 0.002 & 0.002 & $0.01^{* * *}$ & $0.01^{* * *}$ \\
\hline & $(0.002)$ & $(0.002)$ & $(0.002)$ & $(0.002)$ & $(0.002)$ & $(0.002)$ & $(0.002)$ & $(0.002)$ \\
\hline \multirow[t]{2}{*}{ Kyoto protocol } & & & & & $0.52^{* * *}$ & $0.49^{* * *}$ & $0.47^{* * *}$ & $0.45^{* * *}$ \\
\hline & & & & & $(0.073)$ & $(0.073)$ & $(0.078)$ & $(0.079)$ \\
\hline \multirow[t]{2}{*}{ hydro energy } & $-0.24^{* * *}$ & $-0.21^{* * *}$ & $-0.71^{* * *}$ & $-0.69^{* * *}$ & $-0.23^{* * *}$ & $-0.21^{* * *}$ & $-0.72^{* * *}$ & $-0.69^{* * *}$ \\
\hline & $(0.069)$ & $(0.069)$ & $(0.061)$ & $(0.061)$ & $(0.073)$ & $(0.072)$ & $(0.062)$ & $(0.062)$ \\
\hline \multirow[t]{2}{*}{ oil prod'n } & $-0.02^{* * *}$ & $-0.02^{* * *}$ & $-0.02^{* *}$ & $-0.02^{*}$ & $-0.02^{* * *}$ & $-0.02^{* * *}$ & $-0.02^{* *}$ & $-0.02^{* *}$ \\
\hline & $(0.007)$ & $(0.007)$ & $(0.011)$ & $(0.011)$ & $(0.007)$ & $(0.007)$ & $(0.011)$ & $(0.010)$ \\
\hline \multirow[t]{2}{*}{ coal prod'n } & $0.05^{*}$ & $0.06^{* *}$ & $0.08^{* *}$ & $0.09^{* *}$ & $0.04^{*}$ & $0.06^{* *}$ & $0.07^{* *}$ & $0.08^{* *}$ \\
\hline & $(0.026)$ & $(0.027)$ & $(0.037)$ & $(0.039)$ & $(0.025)$ & $(0.026)$ & $(0.035)$ & $(0.037)$ \\
\hline \multirow[t]{2}{*}{ FDI } & & & $-0.04^{* *}$ & $-0.04^{* *}$ & & & $-0.03^{* *}$ & $-0.03^{* *}$ \\
\hline & & & $(0.019)$ & $(0.019)$ & & & $(0.016)$ & $(0.016)$ \\
\hline \multirow[t]{2}{*}{ ODA } & & & $-0.02^{* *}$ & $-0.02^{* *}$ & & & $-0.02^{*}$ & $-0.02^{*}$ \\
\hline & & & $(0.011)$ & $(0.011)$ & & & $(0.012)$ & $(0.012)$ \\
\hline \multirow[t]{2}{*}{ coast } & $0.50^{* * *}$ & $0.51^{* * *}$ & $0.78^{* * * *}$ & $0.78^{* * *}$ & $0.51^{* * * *}$ & $0.53^{* * *}$ & $0.79^{* * *}$ & $0.78^{* * * *}$ \\
\hline & $(0.134)$ & $(0.134)$ & $(0.158)$ & $(0.157)$ & $(0.133)$ & $(0.134)$ & $(0.156)$ & $(0.156)$ \\
\hline \multirow{2}{*}{$\begin{array}{l}\text { regional dummies } \\
\text { time dummies }\end{array}$} & yes & yes & yes & yes & yes & yes & yes & yes \\
\hline & yes & yes & yes & yes & no & no & no & no \\
\hline \multirow[t]{2}{*}{ constant } & $-4.93^{* * *}$ & $-4.67^{* * *}$ & 1.40 & 1.20 & $-4.18^{* * *}$ & $-3.94^{* * *}$ & 2.25 & 2.00 \\
\hline & $(0.456)$ & $(0.468)$ & (1.939) & (1.928) & $(0.373)$ & $(0.384)$ & (1.944) & (1.937) \\
\hline observations & 1,347 & 1,347 & 1,186 & 1,186 & 1,347 & 1,347 & 1,186 & 1,186 \\
\hline $\mathrm{R}$-squared & 0.447 & 0.454 & 0.511 & 0.517 & 0.438 & 0.446 & 0.490 & 0.497 \\
\hline
\end{tabular}

Note: The dependent variable is the computed diversity index.

$* * * \mathrm{p}<0.01, * * \mathrm{p}<0.05, * \mathrm{p}<0.1$ 
For this robustness check, we also use the methods of PPML given there is a substantial number of zero observation in the dependent variable. Table 4.8 presents results using the PPML estimation with per capita electricity generated from nonhydro sources as the dependent variable. Results largely complement our previous findings. Higher income allows countries to generate more nonhydro renewable energy. And again, we would like to highlight the consistent nonlinear effect of income on nonhydro sources of renewable energy depicting a U-shaped kind of relationship. This implies that for a given society during its initial stages of development the use of renewable energy is at large. Traditional societies primary rely on biomass, waste and hydropower as renewable sources of energy. Then as society progresses, more households are entering the energy system shifting to a more energy intensive consumption. Thus, there is a surging increase in the use of fossil fuel replacing the use of renewable energy. However, use of fossil fuel is pollution intensive and harms the environment so, as the society progresses even more, use of clean renewable energy is highly desired. This transition portrays a U-shaped kind of relationship. However, more research has to be done on this issue by investigating this U-shaped relationship not just in the context of developing countries but also with developed countries.

Contrary to Aguirre and Ibikunle (2014) who focuses on OECD and BRICS, we found that energy security is one of the main drivers behind the increasing diffusion of renewable energy in developing countries. The coefficient of energy import is significant and positive. This shows evidence that higher dependence on foreign sources of energy will likely push developing countries to increase energy generation from renewable sources. Even with our previous estimation on diversification, we still a find positive association of increasing energy imports on renewable energy. This is also supported by the positive and significant coefficient of world market price for crude oil. In addition, a higher population growth rate also motivates developing countries to generate more nonhydro renewable energy.

Implementations of renewable energy policies as well as Kyoto Protocol positively influence the generation of nonhydro renewable energy. Surprisingly this time, the estimate of patent and finance variable is negative but not statistically significant. This suggests that the generation of nonhydro renewable energy does not necessarily depend on more technology and access to finance but the diversification of nonhydro sources does. If developing countries decide to generate nonhydro energy this does not necessarily mean diversification. This may explain why the effect of technology and finance is not visible for this estimation with per capita electricity generated from nonhydro sources as the dependent variable. However, if the concern is adapting to varied sources of renewable energy, then more technology and greater access to finance will enhance diversification.

Contrary to our previous results, the coefficient of hydro energy is significant only at $10 \%$ but still the associated relationship is negative. The other control variables behave similarly with our previous findings. Improvement in human capital is positively associated with generation of nonhydro renewable energy. In contrast, higher local oil production does not promote nonhydro renewable energy and this time coal production posted a negative effect but not significant. Developing countries endowed with coastal areas are more likely to generate more nonhydro renewable energy as compared to landlocked countries. 
Table 4.8. Additional robustness check using the Poisson pseudo-maximum likelihood estimation

\begin{tabular}{|c|c|c|c|c|c|c|c|c|}
\hline VARIABLES & $\begin{array}{c}(38) \\
\text { PPML }\end{array}$ & $\begin{array}{c}(39) \\
\text { PPML }\end{array}$ & $\begin{array}{c}(40) \\
\text { PPML }\end{array}$ & $\begin{array}{c}(41) \\
\text { PPML }\end{array}$ & $\begin{array}{c}(42) \\
\text { PPML }\end{array}$ & $\begin{array}{c}(43) \\
\text { PPML }\end{array}$ & $\begin{array}{c}(44) \\
\text { PPML }\end{array}$ & $\begin{array}{c}(45) \\
\text { PPML }\end{array}$ \\
\hline $\log$ GDP pc & $\begin{array}{l}0.53^{* * * *} \\
(0.136)\end{array}$ & $\begin{array}{l}0.49^{* * * *} \\
(0.135)\end{array}$ & $\begin{array}{c}-3.91^{* * *} \\
(0.944)\end{array}$ & $\begin{array}{c}-3.43^{* * *} \\
(0.935)\end{array}$ & $\begin{array}{l}0.54^{* * * *} \\
(0.140)\end{array}$ & $\begin{array}{l}0.50^{* * * *} \\
(0.137)\end{array}$ & $\begin{array}{c}-3.71^{* * *} \\
(0.999)\end{array}$ & $\begin{array}{l}-3.22^{* * * *} \\
(0.981)\end{array}$ \\
\hline $\log$ GDP pc_sq & & & $\begin{array}{l}0.29^{* * * *} \\
(0.064)\end{array}$ & $\begin{array}{l}0.25^{* * * *} \\
(0.065)\end{array}$ & & & $\begin{array}{l}0.27^{* * *} \\
(0.069)\end{array}$ & $\begin{array}{l}0.24^{* * * *} \\
(0.068)\end{array}$ \\
\hline energy import & $\begin{array}{l}0.01^{* * *} \\
(0.001)\end{array}$ & $\begin{array}{l}0.01^{* * * *} \\
(0.001)\end{array}$ & $\begin{array}{l}0.01^{* * *} \\
(0.001)\end{array}$ & $\begin{array}{l}0.01^{* * *} \\
(0.001)\end{array}$ & $\begin{array}{l}0.00^{* * * *} \\
(0.001)\end{array}$ & $\begin{array}{l}0.01^{* * *} \\
(0.001)\end{array}$ & $\begin{array}{l}0.01^{* * * *} \\
(0.001)\end{array}$ & $\begin{array}{l}0.01^{\text {**** }} \\
(0.001)\end{array}$ \\
\hline population growth & $\begin{array}{l}0.38^{* * *} \\
(0.124)\end{array}$ & $\begin{array}{l}0.39^{* * * *} \\
(0.124)\end{array}$ & $\begin{array}{l}0.61^{* * *} \\
(0.154)\end{array}$ & $\begin{array}{l}0.62^{* * *} \\
(0.156)\end{array}$ & $\begin{array}{l}0.38^{* * * *} \\
(0.129)\end{array}$ & $\begin{array}{l}0.39^{* * *} \\
(0.130)\end{array}$ & $\begin{array}{l}0.59^{* * *} \\
(0.164)\end{array}$ & $\begin{array}{l}0.61^{\text {**** }} \\
(0.167)\end{array}$ \\
\hline oil price & $\begin{array}{l}0.02^{* * *} \\
(0.008)\end{array}$ & $\begin{array}{l}0.02^{* * * *} \\
(0.008)\end{array}$ & $\begin{array}{l}0.03^{* * *} \\
(0.008)\end{array}$ & $\begin{array}{l}0.03^{* * *} \\
(0.008)\end{array}$ & $\begin{array}{l}0.01^{* * * *} \\
(0.003)\end{array}$ & $\begin{array}{l}0.01^{* *} \\
(0.003)\end{array}$ & $\begin{array}{l}0.01^{* * *} \\
(0.003)\end{array}$ & $\begin{array}{l}0.01^{* *} \\
(0.003)\end{array}$ \\
\hline policy & & $\begin{array}{l}0.34 * * \\
(0.142)\end{array}$ & & $\begin{array}{c}0.29 * \\
(0.155)\end{array}$ & & $\begin{array}{l}0.38 * * \\
(0.154)\end{array}$ & & $\begin{array}{c}0.33 * * \\
(0.164)\end{array}$ \\
\hline log patents & $\begin{array}{c}0.01 \\
(0.027)\end{array}$ & $\begin{array}{c}-0.01 \\
(0.029)\end{array}$ & $\begin{array}{c}-0.03 \\
(0.029)\end{array}$ & $\begin{array}{c}-0.04 \\
(0.030)\end{array}$ & $\begin{array}{c}-0.00 \\
(0.028)\end{array}$ & $\begin{array}{c}-0.02 \\
(0.030)\end{array}$ & $\begin{array}{c}-0.03 \\
(0.029)\end{array}$ & $\begin{array}{l}-0.05 \\
(0.030)\end{array}$ \\
\hline log finance dev & $\begin{array}{c}-0.04 \\
(0.098)\end{array}$ & $\begin{array}{c}-0.02 \\
(0.100)\end{array}$ & $\begin{array}{c}-0.04 \\
(0.134)\end{array}$ & $\begin{array}{c}-0.01 \\
(0.142)\end{array}$ & $\begin{array}{c}-0.04 \\
(0.100)\end{array}$ & $\begin{array}{l}-0.02 \\
(0.101)\end{array}$ & $\begin{array}{c}-0.09 \\
(0.127)\end{array}$ & $\begin{array}{l}-0.06 \\
(0.131)\end{array}$ \\
\hline sec enrollment & $\begin{array}{l}0.01^{\text {** }} \\
(0.005)\end{array}$ & $\begin{array}{c}0.01^{*} \\
(0.005)\end{array}$ & $\begin{array}{l}0.02^{* * *} \\
(0.005)\end{array}$ & $\begin{array}{l}0.01^{* * *} \\
(0.005)\end{array}$ & $\begin{array}{l}0.01^{* * * *} \\
(0.005)\end{array}$ & $\begin{array}{l}0.01^{* *} \\
(0.005)\end{array}$ & $\begin{array}{l}0.02^{* * *} \\
(0.005)\end{array}$ & $\begin{array}{l}0.02^{* * * *} \\
(0.005)\end{array}$ \\
\hline Kyoto protocol & & & & & $\begin{array}{l}0.59^{* * *} \\
(0.158)\end{array}$ & $\begin{array}{l}0.55^{* * *} \\
(0.156)\end{array}$ & $\begin{array}{l}0.82^{* * *} \\
(0.155)\end{array}$ & $\begin{array}{l}0.79^{* * *} \\
(0.154)\end{array}$ \\
\hline hydro energy & $\begin{array}{l}-0.20^{*} \\
(0.108)\end{array}$ & $\begin{array}{c}-0.15 \\
(0.105)\end{array}$ & $\begin{array}{l}-0.27^{*} \\
(0.143)\end{array}$ & $\begin{array}{c}-0.23 \\
(0.139)\end{array}$ & $\begin{array}{l}-0.20^{*} \\
(0.117)\end{array}$ & $\begin{array}{l}-0.14 \\
(0.112)\end{array}$ & $\begin{array}{l}-0.26^{*} \\
(0.141)\end{array}$ & $\begin{array}{l}-0.21 \\
(0.137)\end{array}$ \\
\hline oil prod'n & $\begin{array}{l}-0.04^{* * *} \\
(0.013)\end{array}$ & $\begin{array}{l}-0.04^{* * *} \\
(0.014)\end{array}$ & $\begin{array}{l}-0.07^{* * *} \\
(0.017)\end{array}$ & $\begin{array}{l}-0.07^{* * *} \\
(0.018)\end{array}$ & $\begin{array}{l}-0.04^{* * *} \\
(0.014)\end{array}$ & $\begin{array}{l}-0.04^{* * *} \\
(0.014)\end{array}$ & $\begin{array}{l}-0.07^{* * *} \\
(0.017)\end{array}$ & $\begin{array}{l}-0.07^{* * * *} \\
(0.018)\end{array}$ \\
\hline coal prod'n & $\begin{array}{l}-0.12 \\
(0.089)\end{array}$ & $\begin{array}{l}-0.10 \\
(0.092)\end{array}$ & $\begin{array}{l}-0.07 \\
(0.103)\end{array}$ & $\begin{array}{l}-0.07 \\
(0.107)\end{array}$ & $\begin{array}{l}-0.11 \\
(0.086)\end{array}$ & $\begin{array}{l}-0.09 \\
(0.090)\end{array}$ & $\begin{array}{l}-0.07 \\
(0.095)\end{array}$ & $\begin{array}{l}-0.06 \\
(0.098)\end{array}$ \\
\hline FDI & & & $\begin{array}{l}-0.12^{* * *} \\
(0.029)\end{array}$ & $\begin{array}{l}-0.12^{* * *} \\
(0.030)\end{array}$ & & & $\begin{array}{l}-0.10^{* * *} \\
(0.029)\end{array}$ & $\begin{array}{l}-0.10^{* * * *} \\
(0.029)\end{array}$ \\
\hline ODA & & & $\begin{array}{l}-0.15^{* * *} \\
(0.042)\end{array}$ & $\begin{array}{l}-0.15^{* * *} \\
(0.042)\end{array}$ & & & $\begin{array}{l}-0.15^{* * *} \\
(0.043)\end{array}$ & $\begin{array}{l}-0.15^{* * * *} \\
(0.042)\end{array}$ \\
\hline coast & $\begin{array}{l}1.58^{* * *} \\
(0.220)\end{array}$ & $\begin{array}{l}1.60^{* * *} \\
(0.223)\end{array}$ & $\begin{array}{l}1.23^{\text {**** }} \\
(0.395)\end{array}$ & $\begin{array}{l}1.22^{* * *} \\
(0.415)\end{array}$ & $\begin{array}{l}1.62^{* * *} \\
(0.216)\end{array}$ & $\begin{array}{l}1.63^{\text {*** }} \\
(0.217)\end{array}$ & $\begin{array}{l}1.20^{* * *} \\
(0.379)\end{array}$ & $\begin{array}{l}1.19^{* * *} \\
(0.401)\end{array}$ \\
\hline $\begin{array}{l}\text { regional dummies } \\
\text { time dummies }\end{array}$ & $\begin{array}{l}\text { yes } \\
\text { yes }\end{array}$ & $\begin{array}{l}\text { yes } \\
\text { yes }\end{array}$ & $\begin{array}{l}\text { yes } \\
\text { yes }\end{array}$ & $\begin{array}{l}\text { yes } \\
\text { yes }\end{array}$ & $\begin{array}{l}\text { yes } \\
\text { no }\end{array}$ & $\begin{array}{l}\text { yes } \\
\text { no }\end{array}$ & $\begin{array}{c}\text { yes } \\
\text { no }\end{array}$ & $\begin{array}{c}\text { yes } \\
\text { no }\end{array}$ \\
\hline constant & $\begin{array}{c}-15.44^{* * *} \\
(1.257)\end{array}$ & $\begin{array}{c}-15.12^{* * *} \\
(1.269)\end{array}$ & $\begin{array}{c}1.32 \\
(3.618)\end{array}$ & $\begin{array}{c}-0.51 \\
(3.605)\end{array}$ & $\begin{array}{c}-14.63^{* * *} \\
(1.048)\end{array}$ & $\begin{array}{c}-14.28^{* * *} \\
(1.075)\end{array}$ & $\begin{array}{c}2.43 \\
(3.739)\end{array}$ & $\begin{array}{c}0.60 \\
(3.666)\end{array}$ \\
\hline observations & 1,383 & 1,383 & 1,221 & 1,221 & 1,383 & 1,383 & 1,221 & 1,221 \\
\hline R-squared & 0.455 & 0.449 & 0.507 & 0.506 & 0.433 & 0.429 & 0.480 & 0.480 \\
\hline
\end{tabular}

Note: The dependent variable used is the per capita electricity generated from nonhydro sources of renewable energy.

$* * * \mathrm{p}<0.01, * * \mathrm{p}<0.05, * \mathrm{p}<0.1$ 


\subsection{Summary and Conclusion}

This paper investigates the motivation behind the rising diversification of nonhydro sources of renewable energy across 117 developing countries for the period 1980-2011. Although there is a substantial number of developing countries not investing in any of the nonhydro sources such as wind, solar, geothermal, biomass and waste, the descriptive statistics indicate that there has been a rapid diversification of nonhydro sources since in the mid-1990s. Hence, we shed some light on this issue by investigating what drives developing countries to adopt various nonhydro sources of renewable energy despite its huge upfront investment cost and other economic, technical and institutional barriers. To capture diversification, we use the number of nonhydro sources and we develop an index measuring diversification. This proposed diversity index represents the weighted number of nonhydro sources using the share of electricity from each particular source to the total electricity generated from nonhydro sources as weights.

Results suggest that higher per capita income is associated with more diversified sources of nonhydro renewable energy. In contrast to most empirical analyses, we include the nonlinear effect of income and found robust evidence suggesting that income has a significant nonlinear effect on the diversification depicting a U-shaped kind of relationship. This implies that at initial stages of development, the adoption of nonhydro renewable energy declines but as the economy grows and accumulates more wealth, developing countries are more likely to invest in various kinds of nonhydro renewable energy. This reflects the non-monotonic influence of income on renewable energy. In terms of energy security, developing countries that are more dependent on foreign sources of energy are more likely to adopt and diversify sources of nonhydro renewable energy. This result is complemented by the positive association of nonhydro renewable energy on rising price for crude oil. Population growth is coupled with rising energy demand and this issue can be potentially addressed with the diversification of nonhydro sources of renewable energy.

Implementation of renewable energy policies, technological innovation, access to finance and accumulation of human capital positively contributes to the diversification of nonhydro sources of renewable energy in developing countries. This confirms Collier and Venables' (2012) argument that developing countries with more technology, developed financial market, skilled human resources and sound governance can move into renewable energy. Meanwhile, abundance of hydropower does not contribute to the diversification of nonhydro source of renewable energy and local abundance of local oil reduces incentives to diversify because developing countries will not have to worry about issues of energy security. The abundance of local oil makes renewable energy more expensive making it less attractive. On the contrary, abundance of coal promotes diversification of renewable energy. This is not what we were expecting but a rather cautious explanation could be that coal complements nonhydro renewable energy. There is also no conclusive evidence that shows FDI and ODA promotes the diversification of nonhydro sources of renewable energy.

Although Kyoto Protocol did not place a heavy burden on developing countries to reduce their emissions, results consistently show significant effect of Kyoto Protocol on the 
diversification. This suggests that global environmental awareness has led to a greater diffusion of renewable energy in developing countries. This can also be associated with the growing number of clean development mechanism (CDM) projects in developing countries. Lastly, developing countries endowed with coastal areas are more likely to diversify sources of nonhydro renewable energy. Coastal areas are relatively windy making it more attractive for investments harnessing wind energy. Also having coastal areas provides ease of access for trading and technology transfer.

We have shed some light on what drives developing countries to diversify sources of nonhydro renewable energy. Developing countries that have greater renewable energy potential are expected to diversify sources. Policy makers should exert a consistent effort in trying to integrate renewable energy in the country's energy mix. Adoption and diversification of nonhydro renewable energy sources is essential for future energy system. While the results are robust, further work in this area can be undertaken. This includes taking into consideration several policy instruments. Despite absence of any binding agreements on reducing carbon emissions, developing countries are increasingly implementing policies promoting renewable energy (Pfeiffer and Mulder 2013). Development of renewable energy does not only depend on natural endowments but with policies supporting its development because market alone will be insufficient to generate more renewable energy. Hence, it is interesting to examine which type of policy instrument such as economic, regulatory, institutional instruments, as well as voluntary approaches promote diversification.

Though results of the study are promising, our conclusion is limited in a sense that we do not identify causality. It will be relevant for policy makers to identify causality. What we did in this paper provides a baseline analysis for diversification by establishing association. By doing this, we assume that the income variable and other control variables are exogenous. However, this may not likely be the case for income because diversification of nonhydro energy may positively affect a developing country's production capacity by supplying additional sources of energy thereby influencing income. However, we can also argue that as of this period since the share of nonhydro renewable energy to the total energy mix in the developing is still relatively small such bias may not be that significant. However, it will still be necessary for future research to untangle this effect. In addition, policy variable is not entirely exogenous because reverse causality might be working. It might be the case that some policies were drawn because of the current state of renewable energy. However, it may also be likely that reverse causality is not valid in situation where policies were needed to encourage investment on renewable energy given that there is substantial number of countries who are yet to integrate renewable energy in their economy. Hence, there is a need to further scrutinize this direction. Despite these limitations, our research still shows salient information in drawing the roadmap for further diffusion of renewable energy in developing countries. Without relying on foreign direct investment and development aid, developing countries with more technology, skilled human capital, developed financial markets, sound governance and natural endowments can move into diversification of nonhydro sources of renewable energy and thereby contribute to the efforts in stabilizing the climate system by reducing global carbon emissions. 


\section{References}

Aguirre, Mariana, and Gbenga Ibikunle. 2014. "Determinants of Renewable Energy Growth: A Global Sample Analysis.” Energy Policy 69 (June): 374-84. doi:10.1016/j.enpol.2014.02.036.

Ala-Mantila, Sanna, Jukka Heinonen, and Seppo Junnila. 2014. "Relationship between Urbanization, Direct and Indirect Greenhouse Gas Emissions, and Expenditures: A Multivariate Analysis." Ecological Economics 104 (August): 129-39. doi:10.1016/j.ecolecon.2014.04.019.

Albert, Jose Ramon, and Andre Philippe Ramos. 2010. "Examining Recent Trends in Poverty, Inequality, and Vulnerability.” PIDS Policy Notes 2010 (03). http://dirp4.pids.gov.ph/ris/pn/pidspn1003.pdf.

Alcantara, Vicent, and Juan Antonio Duro. 2004. "Inequality of Energy Intensities across OECD Countries: A Note.” Energy Policy 32 (11): 1257-60. doi:10.1016/S0301-4215(03)000958.

Allison, Paul, and Richard Walterman. 2002. "Fixed-Effects Negative Binomial Regression Models." Sociological Methodology 32: 247-65.

Ang, B. W, F. Q Zhang, and Ki-Hong Choi. 1998. "Factorizing Changes in Energy and Environmental Indicators through Decomposition.” Energy 23 (6): 489-95. doi:10.1016/S0360-5442(98)00016-4.

Ang, B. W., and F. L. Liu. 2001. "A New Energy Decomposition Method: Perfect in Decomposition and Consistent in Aggregation." Energy 26 (6): 537-48. doi:10.1016/S0360-5442(01)00022-6.

Ang, B.W. 2004. "Decomposition Analysis for Policymaking in Energy:: Which Is the Preferred Method?” Energy Policy 32 (9): 1131-39. doi:10.1016/S0301-4215(03)00076-4.

Asian Development Bank [ADB]. 2014. "Asian Development Outlook 2014: Philippine Economy." http://www.adb.org/countries/philippines/economy.

Baiocchi, Giovanni, Jan Minx, and Klaus Hubacek. 2010. "The Impact of Social Factors and Consumer Behavior on Carbon Dioxide Emissions in the United Kingdom." Journal of Industrial Ecology 14 (1): 50-72. doi:10.1111/j.1530-9290.2009.00216.x.

Barkley, Andrew, Hikaru Hawana Peterson, and James Shroyer. 2010. "Wheat Variety Selection to Maximize Returns and Minimize Risk: An Application of Portfolio Theory." Journal of Agricultural \& Applied Economics 42 (1): 39.

Baumert, Kevin A, Timothy Herzog, and Jonathan Pershing. 2005. Navigating the Numbers: Greenhouse Gas Data and International Climate Policy. Washington, D.C.: World Resources Institute.

Bhattacharyya, Subhes C., and Wataru Matsumura. 2010. "Changes in the GHG Emission Intensity in EU-15: Lessons from a Decomposition Analysis.” Energy 35 (8): 3315-22. doi:10.1016/j.energy.2010.04.017.

Bin, Shui, and Hadi Dowlatabadi. 2005. "Consumer Lifestyle Approach to US Energy Use and the Related CO2 Emissions." Energy Policy 33 (2): 197-208. doi:10.1016/S03014215(03)00210-6.

Blenkinsopp, T., S. R Coles, and K. Kirwan. 2013. "Renewable Energy for Rural Communities in Maharashtra, India.” Energy Policy 60 (September): 192-99. doi:10.1016/j.enpol.2013.04.077.

Brunnschweiler, Christa N. 2010. "Finance for Renewable Energy: An Empirical Analysis of Developing and Transition Economies." Environment and Development Economics 15 (03): 241-74. doi:10.1017/S1355770X1000001X.

Büchs, Milena, and Sylke V. Schnepf. 2013. "Who Emits Most? Associations between SocioEconomic Factors and UK Households' Home Energy, Transport, Indirect and Total CO2 
Emissions." Ecological Economics 90 (June): 114-23.

doi:10.1016/j.ecolecon.2013.03.007.

Cameron, A. Colin, and Pravin Trivedi. 1998. Regression Analysis of Count Data. Vol.

Cambridge University Press. Cambridge, NY.

http://www.econ.ucdavis.edu/faculty/cameron/racd/count.html

. 2010. Micoeconomics Using Stata (Revised Ed.). College Station, Texas: Stata Press.

Cameron, A. Colin, and Pravin K. Trivedi. 1986. "Econometric Models Based on Count Data. Comparisons and Applications of Some Estimators and Tests." Journal of Applied Econometrics 1 (1): 29-53. doi:10.1002/jae.3950010104.

Cantore, Nicola, and Emilio Padilla. 2010. "Equality and CO2 Emissions Distribution in Climate Change Integrated Assessment Modelling." Energy 35 (1): 298-313. doi:10.1016/j.energy.2009.09.022.

Carley, Sanya. 2009. "State Renewable Energy Electricity Policies: An Empirical Evaluation of Effectiveness.” Energy Policy 37 (8): 3071-81. doi:10.1016/j.enpol.2009.03.062.

Chang, Ting-Huan, Chien-Ming Huang, and Ming-Chih Lee. 2009. "Threshold Effect of the Economic Growth Rate on the Renewable Energy Development from a Change in Energy Price: Evidence from OECD Countries.” Energy Policy 37 (12): 5796-5802. doi:10.1016/j.enpol.2009.08.049.

Clarke-Sather, Afton, Jiansheng Qu, Qin Wang, Jingjing Zeng, and Yan Li. 2011. "Carbon Inequality at the Sub-National Scale: A Case Study of Provincial-Level Inequality in CO2 Emissions in China 1997-2007." Energy Policy 39 (9): 5420-28. doi:10.1016/j.enpol.2011.05.021.

Collier, Paul, and Anthony J. Venables. 2012. "Greening Africa? Technologies, Endowments and the Latecomer Effect." Energy Economics, Green Perspectives, 34, Supplement 1 (November): S75-S84. doi:10.1016/j.eneco.2012.08.035.

Corong, Erwin. 2008. "Contributor's Report on Philippine Input-Output Table for GTAP Data Base.” https://www.gtap.agecon.purdue.edu/resources/download/4089.pdf.

Cox, Adam, Alan Collins, Lee Woods, and Neil Ferguson. 2012. "A Household Level Environmental Kuznets Curve? Some Recent Evidence on Transport Emissions and Income.” Economics Letters 115 (2): 187-89. doi:10.1016/j.econlet.2011.12.014.

Cropper, Maureen, and Charles Griffiths. 1994. "The Interaction of Population Growth and Environmental Quality." The American Economic Review, 250-54.

Deaton, Angus. 1992. Understanding Consumption. Oxford University Press.

Department of Energy. 2013. "Renewable Energy Tables and Figures." http://www.doe.gov.ph/renewable-energy-res/renewable-energy-tables-and-figures/284re-share-in-total-primary-energy-consumption.

Department of Energy, Philippines. 2014. "Empowering the Nation through Renewable Energy." https://www.doe.gov.ph/renewable-energy-res/.

Dinda, Soumyananda. 2004. "Environmental Kuznets Curve Hypothesis: A Survey." Ecological Economics 49 (4): 431-55. doi:10.1016/j.ecolecon.2004.02.011.

Dong, C. G. 2012. "Feed-in Tariff vs. Renewable Portfolio Standard: An Empirical Test of Their Relative Effectiveness in Promoting Wind Capacity Development." Energy Policy 42: 476-85. doi:10.1016/j.enpol.2011.12.014.

Dow, William H., and Edward C. Norton. 2003. "Choosing Between and Interpreting the Heckit and Two-Part Models for Corner Solutions." Health Services and Outcomes Research Methodology 4 (1): 5-18. doi:10.1023/A:1025827426320.

Druckman, Angela, and Tim Jackson. 2008. "Household Energy Consumption in the UK: A Highly Geographically and Socio-Economically Disaggregated Model.” Energy Policy 36 (8): 3177-92. doi:10.1016/j.enpol.2008.03.021. . 2009. "The Carbon Footprint of UK Households 1990-2004: A Socio-Economically Disaggregated, Quasi-Multi-Regional Input-output Model.” Ecological Economics 68 (7): 2066-77. doi:10.1016/j.ecolecon.2009.01.013. 
Duan, Naihua, Willard G. Manning, Carl N. Morris, and Joseph P. Newhouse. 1983. "A Comparison of Alternative Models for the Demand for Medical Care." Journal of Business \& Economic Statistics 1 (2): 115-26. doi:10.1080/07350015.1983.10509330.

Dulal, Hari Bansha, Kalim U. Shah, Chandan Sapkota, Gengaiah Uma, and Bibek R. Kandel. 2013. "Renewable Energy Diffusion in Asia: Can It Happen without Government Support?" Energy Policy 59 (August): 301-11. doi:10.1016/j.enpol.2013.03.040.

Duro, Juan Antonio. 2012. "On the Automatic Application of Inequality Indexes in the Analysis of the International Distribution of Environmental Indicators." Ecological Economics 76 (April): 1-7. doi:10.1016/j.ecolecon.2011.12.019.

Duro, Juan Antonio, and Emilio Padilla. 2006. "International Inequalities in per Capita CO2 Emissions: A Decomposition Methodology by Kaya Factors." Energy Economics 28 (2): 170-87. doi:10.1016/j.eneco.2005.12.004.

Duro, Juan Antonio, and Jordi Teixidó-Figueras. 2013. "Ecological Footprint Inequality across Countries: The Role of Environment Intensity, Income and Interaction Effects." Ecological Economics 93: 34-41.

Eberhard, Anton, Orvika Rosnes, Maria Shkaratan, and Haakon Vennemo. 2011. Africa's Power Infrastructure: Investment, Integration, Efficiency. The World Bank. http://elibrary.worldbank.org/doi/book/10.1596/978-0-8213-8455-8.

Ericta, Carmelita, and Emma Fabian. 2009. A Documentation of the Philippines' Family Income and Expenditure Survey. Philippine Institute for Development Studies. http://core.kmi.open.ac.uk/download/pdf/6412342.pdf.

Estudillo, Jonna P. 1997. "Income Inequality in the Philippines, 1961-91." The Developing Economies 35 (1): 68-95.

Fahmy, Eldin, Joshua Thumim, and Vicki White. 2011. "The Distribution of UK Household CO2 Emissions: Interim Report." JRF Programme Paper: Climate Change and Social Justice, University of Bristol and Centre for Sustainable Energy. http://www.jrf.org.uk/sites/files/jrf/carbon-reduction-policy-full.pdf.

Figge, Frank. 2004. "Bio-Folio: Applying Portfolio Theory to Biodiversity." Biodiversity \& Conservation 13 (4): 827-49. doi:10.1023/B:BIOC.0000011729.93889.34.

Filmer, Deon, and Lant H. Pritchett. 2001. "Estimating Wealth Effects Without Expenditure Data-Or Tears: An Application To Educational Enrollments In States Of India." Demography 38 (1): 115-32. doi:10.1353/dem.2001.0003.

Frondel, Manuel, and Colin Vance. 2010. "Driving for Fun? Comparing the Effect of Fuel Prices on Weekday and Weekend Fuel Consumption." Energy Economics 32 (1): 102-9. doi:10.1016/j.eneco.2009.08.020. . 2012. On Interaction Effects: The Case of Heckit and Two-Part Models. SSRN Scholarly Paper ID 1990166. Rochester, NY: Social Science Research Network. http://papers.ssrn.com/abstract=1990166.

Galeotti, Marzio, Matteo Manera, and Alessandro Lanza. 2008. "On the Robustness of Robustness Checks of the Environmental Kuznets Curve Hypothesis." Environmental and Resource Economics 42 (4): 551-74. doi:10.1007/s10640-008-9224-x.

Germanwatch. 2014. "Global Climate Risk Index.” http://germanwatch.org/en/cri.

Girod, Bastien, and Peter De Haan. 2010. "More or Better? A Model for Changes in Household Greenhouse Gas Emissions due to Higher Income.” Journal of Industrial Ecology 14 (1): 31-49. doi:10.1111/j.1530-9290.2009.00202.x.

Golley, Jane, and Xin Meng. 2012. "Income Inequality and Carbon Dioxide Emissions: The Case of Chinese Urban Households." Energy Economics 34 (6): 1864-72. doi:10.1016/j.eneco.2012.07.025.

Golub, Stephen, Celine Kauffmann, and Philipp Yeres. 2011. "Defining and Measuring Green FDI: An Exploratory Review of Existing Work and Evidence." OECD Working Papers on International Investment, No. 2011/2, OECD Investment Division, 
Www.oecd.org/daf/investment/workingpapers.

http://www.oecd.org/daf/inv/internationalinvestmentagreements/48171454.pdf.

Grogger, Jeffrey T., and Richard T. Carson. 1991. "Models for Truncated Counts." Journal of Applied Econometrics 6 (3): 225-38.

Groot, Loek. 2010. “Carbon Lorenz Curves.” Resource and Energy Economics 32 (1): 45-64. doi:10.1016/j.reseneeco.2009.07.001.

Grossman, Gene M., and Alan B. Krueger. 1991. Environmental Impacts of a North American Free Trade Agreement. Working Paper 3914. National Bureau of Economic Research. http://www.nber.org/papers/w3914.

Grunewald, Nicole, Michael Jakob, and Ioanna Mouratiadou. 2014. "Decomposing Inequality in CO2 Emissions: The Role of Primary Energy Carriers and Economic Sectors." Ecological Economics 100 (April): 183-94. doi:10.1016/j.ecolecon.2014.02.007.

GTAP. 2011. "Center for Global Trade Analysis, GTAP Data Bases: Two Concordances." https://www.gtap.agecon.purdue.edu/databases/contribute/concordinfo.asp.

Guimarães, Paulo. 2008. "The Fixed Effects Negative Binomial Model Revisited." Economics Letters 99 (1): 63-66. doi:10.1016/j.econlet.2007.05.030.

Gwatkin, Davidson R., Shea Rutstein, Kiersten Johnson, Eldaw Suliman, Adam Wagstaff, and Agbessi Amouzou. 2007. Socio-Economic Differences in Health, Nutrition, and Population within Developing Countries: An Overview. The World Bank: Health, Nutrition and Population.

Hay, Joel W., and Randall J. Olsen. 1984. "Let Them Eat Cake: A Note on Comparing Alternative Models of the Demand for Medical Care." Journal of Business \& Economic Statistics 2 (3): 279-82. doi:10.2307/1391710.

Hedenus, Fredrik, and Christian Azar. 2005. "Estimates of Trends in Global Income and Resource Inequalities." Ecological Economics 55 (3): 351-64. doi:10.1016/j.ecolecon.2004.10.004.

Heerink, Nico, Abay Mulatu, and Erwin Bulte. 2001. "Income Inequality and the Environment: Aggregation Bias in Environmental Kuznets Curves.” Ecological Economics 38 (3): 35967. doi:10.1016/S0921-8009(01)00171-9.

Heil, Mark T., and Quentin T. Wodon. 1997. "Inequality in CO2 Emissions Between Poor and Rich Countries." The Journal of Environment \& Development 6 (4): 426-52. doi:10.1177/107049659700600404. . 2000. "Future Inequality in CO2 Emissions and the Impact of Abatement Proposals." Environmental and Resource Economics 17 (2): 163-81. doi:10.1023/A:1008326515058.

Hentschel, Jesko, and Peter Lanjouw. 1996. "Constructing an Indicator of Consumption for the Analysis of Poverty: Principles and Illustrations with Reference to Ecuador." Living Standards Measurement Study Working Paper No. 124. The World Bank, Washington, D.C.

Hertwich, Edgar G., and Glen P. Peters. 2009. "Carbon Footprint of Nations: A Global, TradeLinked Analysis.” Environmental Science \& Technology 43 (16): 6414-20. doi:10.1021/es803496a.

IEA. 2013. International Energy Agency (IEA): CO2 Emissions from Fuel Combustion. http://www.iea.org/publications/freepublications/publication/co2emissionsfromfuelcombu stionhighlights2013.pdf. . 2014. "IEA - Renewable Energy." http://www.iea.org/policiesandmeasures/renewableenergy/. . 2014. "CO2 Emissions Statistics.” Accessed August 2. http://www.iea.org/statistics/topics/CO2emissions/.

IPCC. 2005. "IPCC WGIII: Special Report on Carbon Dioxide Capture and Storage." http://www.langtoninfo.com/web_content/9780521685511_frontmatter.pdf. 2012. Special Report of the IPCC on Renewable Energy Sources and Climate Change Mitigation. Cambridge University Press, New York. http://srren.ipccwg3.de/report/IPCC_SRREN_Full_Report.pdf. 
. 2013a. "Intergovernmental Panel on Climate Chnge (IPCC) Press Release - Human Influence on Climate Clear."

http://www.ipcc.ch/news_and_events/docs/ar5/press_release_ar5_wgi_en.pdf.

_. 2013b. "IPCC - Task Force on National Greenhouse Gas Inventories." http://www.ipccnggip.iges.or.jp/public/gl/invs1.html.

- 2013c. "Emissions Scenarios."

http://www.ipcc.ch/ipccreports/sres/emission/index.php?idp=50.

Jacquemin, Alexis P., and Charles H. Berry. 1979. "Entropy Measure of Diversification and Corporate Growth.” The Journal of Industrial Economics 27 (4): 359-69. doi:10.2307/2097958.

Johnstone, Nick, Ivan Hascic, and David Popp. 2010. "Renewable Energy Policies and Technological Innovation: Evidence Based on Patent Counts.” Environmental and Resource Economics 45 (1): 133-55. doi:10.1007/s10640-009-9309-1.

Kahn, Matthew E. 1998. “A Household Level Environmental Kuznets Curve.” Economics Letters 59 (2): 269-73.

Kakwani, Nanak C. 1977. "Measurement of Tax Progressivity: An International Comparison." The Economic Journal 87 (345): 71-80.

Kakwani, Nanak, Adam Wagstaff, and Eddy van Doorslaer. 1997. "Socioeconomic Inequalities in Health: Measurement, Computation, and Statistical Inference." Journal of Econometrics 77 (1): 87-103. doi:10.1016/S0304-4076(96)01807-6.

Kaya, Yoichi. 1995. "The Role of CO2 Removal and Disposal." Energy Conservation and Management 36 (6-9): 375-80.

Kenny, Tricia, and N.F. Gray. 2009. "A Preliminary Survey of Household and Personal Carbon Dioxide Emissions in Ireland." Environment International 35 (2): 259-72. doi:10.1016/j.envint.2008.06.008.

Kerkhof, Annemarie C., René M.J. Benders, and Henri C. Moll. 2009. "Determinants of Variation in Household CO2 Emissions between and within Countries." Energy Policy 37 (4): 1509-17. doi:10.1016/j.enpol.2008.12.013.

Kerkhof, Annemarie C., Sanderine Nonhebel, and Henri C. Moll. 2009. "Relating the Environmental Impact of Consumption to Household Expenditures: An Input-output Analysis." Ecological Economics 68 (4): 1160-70. doi:10.1016/j.ecolecon.2008.08.004.

Klasen, Stephan. 1997. "Poverty, Inequality and Deprivation in South Africa: An Analysis of the 1993 SALDRU Survey." Social Indicators Research 41 (1-3): 51-94. doi:10.1023/A:1006892216864.

Kok, Rixt, René M.J. Benders, and Henri C. Moll. 2006. "Measuring the Environmental Load of Household Consumption Using Some Methods Based on Input-output Energy Analysis: A Comparison of Methods and a Discussion of Results." Energy Policy 34 (17): 2744-61. doi:10.1016/j.enpol.2005.04.006.

Kolenikov, Stanislav, and Gustavo Angeles. 2009. "Socio-Economic Status Measurement with Discrete Proxy Variables: Is Principal Component Analysis a Reliable Answer?" Review of Income and Wealth 55 (1): 128-65. doi:10.1111/j.1475-4991.2008.00309.x.

Kuznets, Simon. 1955. "Economic Growth and Income Inequality." The American Economic Review, 1-28.

Lee, Huey-Lin. 2008. "An Emissions Data Base for Integrated Assessment of Climate Change Policy Using GTAP." Center for Global Trade Analysis. http://www.gtap.agecon.purdue.edu/resources/res_display.asp?RecordID=1143.

Lenzen, Manfred. 1998a. "Primary Energy and Greenhouse Gases Embodied in Australian Final Consumption: An Input-output Analysis." Energy Policy 26 (6): 495-506. . 1998b. "Energy and Greenhouse Gas Cost of Living for Australia during 1993/94." Energy 23 (6): 497-516. doi:10.1016/S0360-5442(98)00020-6.

Lenzen, Manfred, Mette Wier, Claude Cohen, Hitoshi Hayami, Shonali Pachauri, and Roberto Schaeffer. 2006. "A Comparative Multivariate Analysis of Household Energy 
Requirements in Australia, Brazil, Denmark, India and Japan.” Energy 31 (2-3): 181-207. doi:10.1016/j.energy.2005.01.009.

Lerman, Robert I., and Shlomo Yitzhaki. 1985. "Income Inequality Effects by Income Source: A New Approach and Applications to the United States." The Review of Economics and Statistics 67 (1): 151-56. doi:10.2307/1928447.

Leung, Siu Fai, and Shihti Yu. 1996. "On the Choice between Sample Selection and Two-Part Models.” Journal of Econometrics 72 (1-2): 197-229. doi:10.1016/0304-4076(94)017204.

Levy, Amnon, Khorshed Chowdhury, and Jonathan Livermore. 2009. "International Emission Inequality: Abatement on a per Capita Basis with Rewards.” http://ro.uow.edu.au/commwkpapers/216/.

Li, Xianguo. 2005. "Diversification and Localization of Energy Systems for Sustainable Development and Energy Security.” Energy Policy 33 (17): 2237-43. doi:10.1016/j.enpol.2004.05.002.

Lintner, John. 1965. "Security Prices, Risk, and Maximal Gains From Diversification." The Journal of Finance 20 (4): 587-615. doi:10.2307/2977249.

Lise, Wietze. 2006. "Decomposition of CO2 Emissions over 1980-2003 in Turkey." Energy Policy 34 (14): 1841-52. doi:10.1016/j.enpol.2004.12.021.

Lopez-Feldman, Alejandro. 2006. "Decomposing Inequality and Obtaining Marginal Effects." The Stata Journal 6 (1): 106-11.

Manning, W. G., N. Duan, and W. H. Rogers. 1987. "Monte Carlo Evidence on the Choice between Sample Selection and Two-Part Models." Journal of Econometrics 35 (1): 59-82. doi:10.1016/0304-4076(87)90081-9.

Markowitz, Harry. 1952. "Portfolio Selection." The Journal of Finance 7 (1): 77-91. doi:10.2307/2975974. 1959. Portfolio Selection: Efficient Diversification of Investments. New York: John Wiley \& Sons, Inc. http://cowles.econ.yale.edu/P/cm/m16/.

Marques, António C., José A. Fuinhas, and J.R. Pires Manso. 2010. "Motivations Driving Renewable Energy in European Countries: A Panel Data Approach.” Energy Policy 38 (11): 6877-85. doi:10.1016/j.enpol.2010.07.003.

Marques, António Cardoso, and José Alberto Fuinhas. 2012. "Are Public Policies towards Renewables Successful? Evidence from European Countries." Renewable Energy 44 (August): 109-18. doi:10.1016/j.renene.2012.01.007.

Martínez-Zarzoso, Inmaculada. 2013. "The Log of Gravity Revisited.” Applied Economics 45 (3): 311-27. doi:10.1080/00036846.2011.599786.

Martinez-Zarzoso, Inmaculada, Felicitas Nowak-Lehmann, and Sebastian Vollmer. 2007. "The Log of Gravity Revisited." CeGE Discussion Paper 64, University of Göttingen. http://www.unigoettingen.de/de/sh/43876.htmlde/document/download/772ff7ff216fd23b2f44191df59607 e7.pdf/WP008.pdf.

Menz, Fredric C., and Stephan Vachon. 2006. "The Effectiveness of Different Policy Regimes for Promoting Wind Power: Experiences from the States.” Energy Policy 34 (14): 1786-96. doi:10.1016/j.enpol.2004.12.018.

Minx, J.C., T. Wiedmann, R. Wood, G.P. Peters, M. Lenzen, A. Owen, K. Scott, et al. 2009. "Input-Output Analysis and Carbon Footprinting: An Overview of Applications." Economic Systems Research 21 (3): 187-216. doi:10.1080/09535310903541298.

NSCB. 2003. "Resolution No. 9 - Adoption Of The Operational Definition Of Urban Areas In The Philippines." http://www.nscb.gov.ph/resolutions/2003/9.asp. . 2014. "NSCB - Technical Notes - Input-Output Tables of the Philippines (IO)." http://www.nscb.gov.ph/technotes/io_tech.asp. 
Ohler, Adrienne, and Ian Fetters. 2014. "The Causal Relationship between Renewable Electricity Generation and GDP Growth: A Study of Energy Sources.” Energy Economics 43: 12539. doi:10.1016/j.eneco.2014.02.009.

Pachauri, Shonali, and Daniel Spreng. 2002. "Direct and Indirect Energy Requirements of Households in India." Energy Policy 30 (6): 511-23.

Padilla, Emilio, and Juan Antonio Duro. 2013. "Explanatory Factors of CO2 per Capita Emission Inequality in the European Union.” Energy Policy 62 (November): 1320-28. doi:10.1016/j.enpol.2013.07.018.

Padilla, Emilio, and Alfredo Serrano. 2006. "Inequality in CO2 Emissions across Countries and Its Relationship with Income Inequality: A Distributive Approach.” Energy Policy 34 (14): 1762-72. doi:10.1016/j.enpol.2004.12.014.

Painuly, Jyoti Prasad, and Norbert Wohlgemuth. 2006. "Renewable Energy Financing - What Can We Learn from Experience in Developing Countries?” Energy Studies Review 14 (2): 154-70.

Parikh, Jyoti, Manoj Panda, and N. S. Murthy. 1997. "Consumption Patterns by Income Groups and Carbon-Dioxide Implications for India: 1990-2010." International Journal of Global Energy Issues 9 (4-6). http://www.popline.org/node/529302.

Pfeiffer, Birte, and Peter Mulder. 2013. "Explaining the Diffusion of Renewable Energy Technology in Developing Countries." Energy Economics 40 (November): 285-96. doi:10.1016/j.eneco.2013.07.005.

Popp, David. 2011. "International Technology Transfer, Climate Change, and the Clean Development Mechanism." Review of Environmental Economics and Policy 5 (1): 13152. doi:10.1093/reep/req018.

Popp, David, Ivan Hascic, and Neelakshi Medhi. 2011. "Technology and the Diffusion of Renewable Energy." Energy Economics, Special Issue on The Economics of Technologies to Combat Global Warming, 33 (4): 648-62. doi:10.1016/j.eneco.2010.08.007.

Rees, William E. 1992. "Ecological Footprints and Appropriated Carrying Capacity: What Urban Economics Leaves out." Environment and Urbanization 4 (2): 121-30. doi:10.1177/095624789200400212.

REN21. 2013. "Renewables Global Status Report." http://www.ren21.net/REN21Activities/GlobalStatusReport.aspx.

Robison, Lindon J., and John R. Brake. 1979. "Application of Portfolio Theory to Farmer and Lender Behavior.” American Journal of Agricultural Economics 61 (1): 158-64. doi:10.2307/1239520.

Rosa, Eugene A., and Thomas Dietz. 2012. "Human Drivers of National Greenhouse-Gas Emissions." Nature Climate Change 2 (8): 581-86. doi:10.1038/nclimate1506.

Sadorsky, Perry. 2009a. "Renewable Energy Consumption, CO2 Emissions and Oil Prices in the G7 Countries." Energy Economics 31 (3): 456-62. doi:10.1016/j.eneco.2008.12.010. . 2009b. "Renewable Energy Consumption and Income in Emerging Economies." Energy Policy 37 (10): 4021-28. doi:10.1016/j.enpol.2009.05.003.

Saidi, Lotfi, and Farhat Fnaiech. 2014. "Experiences in Renewable Energy and Energy Efficiency in Tunisia: Case Study of a Developing Country." Renewable and Sustainable Energy Reviews 32 (April): 729-38. doi:10.1016/j.rser.2014.01.045.

Salim, Ruhul A., and Shuddhasattwa Rafiq. 2012. "Why Do Some Emerging Economies Proactively Accelerate the Adoption of Renewable Energy?" Energy Economics 34 (4): 1051-57. doi:10.1016/j.eneco.2011.08.015.

Santos Silva, J. M. C., and Silvana Tenreyro. 2006. "The Log of Gravity." The Review of Economics and Statistics 88 (4): 641-58.

. 2011. "Further Simulation Evidence on the Performance of the Poisson PseudoMaximum Likelihood Estimator." Economics Letters 112 (2): 220-22. doi:10.1016/j.econlet.2011.05.008. 
Stark, Oded, J. Edward Taylor, and Shlomo Yitzhaki. 1986. "Remittances and Inequality." The Economic Journal 96 (383): 722-40.

Steckel, Jan Christoph, Michael Jakob, Robert Marschinski, and Gunnar Luderer. 2011. "From Carbonization to decarbonization?-Past Trends and Future Scenarios for China's CO2 Emissions.” Energy Policy 39 (6): 3443-55. doi:10.1016/j.enpol.2011.03.042.

Stern, David I. 2004. "The Rise and Fall of the Environmental Kuznets Curve." World Development 32 (8): 1419-39. doi:10.1016/j.worlddev.2004.03.004.

Sun, J.W. 1999. "Decomposition of Aggregate CO2 Emissions in the OECD: 1960-1995." Energy Journal 20: 147-55.

Thiam, Djiby Racine. 2011. "An Energy Pricing Scheme for the Diffusion of Decentralized Renewable Technology Investment in Developing Countries.” Energy Policy 39 (7): 4284-97. doi:10.1016/j.enpol.2011.04.046.

Tobin, J. 1958. "Liquidity Preference as Behavior Towards Risk." The Review of Economic Studies 25 (2): 65-86. doi:10.2307/2296205.

Tran, Nhuong, Norbert Wilson, and Diane Hite. 2013. "Choosing the Best Model in the Presence of Zero Trade: A Fish Product Analysis." Frontiers of Economics and Globalization 12: $127-48$.

United Nations Conference on Trade and Development [UNCTAD]. 2013. "Foreign direct investment to Africa increases, defying global trend for 2012". http://unctad.org/en/pages/PressRelease.aspx?OriginalVersionID=136

United Nations Framework Convention on Climate Change [UNFCCC]. 2013. "Status of Ratification.” http://unfccc.int/essential_background/convention/status_of_ratification /items/2631.php. . 2014. "Clean Development Mechanism, Unite Nations Framework Convention on Climate Change." http://cdm.unfccc.int/about/index.html.

United Nations Development Programme [UNDP]. 2012. "Asia-Pacific Must Manage Emissions Now While Helping Poor." http://www.undp.org/content/undp/en/home/presscenter/pressreleases/2012/05/10/asiapacific-can-no-longer-afford-to-grow-first-and-clean-up-later-says-undp-report/.

US EIA. 2011. "International Energy Outlook 2011." http://www.eia.gov/forecasts/ieo/pdf/0484(2011).pdf. . 2013. US Energy Information Administration (US EIA): International Energy Outlook 2013 With Projections to 2040. Government Printing Office.

Vachon, Stephan, and Fredric C. Menz. 2006. "The Role of Social, Political, and Economic Interests in Promoting State Green Electricity Policies." Environmental Science \& Policy 9 (7-8): 652-62. doi:10.1016/j.envsci.2006.07.005.

Van Hove, Leo. 1993. "Diversification of Primary Energy Consumption in Six West European Countries: Quantification and Analysis by Means of Measures of Concentration." Energy Economics 15 (4): 239-44. doi:10.1016/0140-9883(93)90013-H.

Verbruggen, Aviel, Manfred Fischedick, William Moomaw, Tony Weir, Alain Nadaï, Lars J. Nilsson, John Nyboer, and Jayant Sathaye. 2010. "Renewable Energy Costs, Potentials, Barriers: Conceptual Issues.” Energy Policy 38 (2): 850-61. doi:10.1016/j.enpol.2009.10.036.

Vinuya, Ferdinand, Ferdinand DiFurio, and Erica Sandoval. 2010. "A Decomposition Analysis of CO2 Emissions in the United States.” Applied Economics Letters 17 (10): 925-31. doi:10.1080/00036840902762688.

Wackernagel, Mathis, and William E. Rees. 1996. Our Ecological Footprint. Reducing Human Impact on Earth. New Society Press. http://www.newsociety.com/Books/O/OurEcological-Footprint.

Ward, Karen. 2012. "The World in 2050." Quantifying the Shift in the Global Economy. HSBC. https://161.113.4.87/1/PA_1_083Q9FJ08A002FBP5S00000000/content/usshared/Premier /Promotions/2012/Jan/pdf/The_World_in_2050.pdf. 
Watson, Jim, and Raphael Sauter. 2011. "Sustainable Innovation through Leapfrogging: A Review of the Evidence." International Journal of Technology and Globalisation 5 (3/4): 170. doi:10.1504/IJTG.2011.039763.

WDI. 2014. "World Development Indicators." http://data.worldbank.org/data-catalog/worlddevelopment-indicators.

Weber, Christopher, and Scott Matthews. 2008. "Quantifying the Global and Distributional Aspects of American Household Carbon Footprint." Ecological Economics 66 (2-3): 379_ 91. doi:10.1016/j.ecolecon.2007.09.021.

Weber, Christopher, and Adriaan Perrels. 2000. "Modelling Lifestyle Effects on Energy Demand and Related Emissions.” Energy Policy 28 (8): 549-66.

Weidema, Bo P., Mikkel Thrane, Per Christensen, Jannick Schmidt, and Søren Løkke. 2008. "Carbon Footprint." Journal of Industrial Ecology 12 (1): 3-6. doi:10.1111/j.15309290.2008.00005.x.

Wiedmann, Thomas, and Jan Minx. 2007. “A Definition of 'Carbon Footprint."” Ecological Economics Research Trends 1: 1-11.

World Bank. 2010. "A Strategic Approach to Climate Change in the Philippines: An Assessment of Low-Carbon Interventions in the Transport and Power Sectors" http://siteresources.worldbank.org/INTPHILIPPINES/Resources/PH_Low_Carbon_Trans port_and_Power.pdf . 2013. "CO2 Emissions (metric Tons per Capita) | Data | Table." http://data.worldbank.org/indicator/EN.ATM.CO2E.PC/countries?display=default.

$\mathrm{Xu}, \mathrm{X} . Y$., and B.W. Ang. 2013. "Index Decomposition Analysis Applied to CO2 Emission Studies." Ecological Economics 93 (September): 313-29. doi:10.1016/j.ecolecon.2013.06.007.

Yaguchi, Yue, Tetsushi Sonobe, and Keijiro Otsuka. 2007. "Beyond the Environmental Kuznets Curve: A Comparative Study of SO2 and CO2 Emissions between Japan and China." Environment and Development Economics 12 (03): 445-70. doi:10.1017/S1355770X07003592.

Zhao, Yong, Kam Ki Tang, and Li-li Wang. 2013. "Do Renewable Electricity Policies Promote Renewable Electricity Generation? Evidence from Panel Data." Energy Policy 62 (November): 887-97. doi:10.1016/j.enpol.2013.07.072.

Zheng, Siqi, Rui Wang, Edward Glaeser, and Mathew Kahn. 2010. "The Greenness of China: Household Carbon Dioxide Emissions and Urban Development." Harvard Environmental Economics Program, no. Discussion Paper 2010-12 (January). http://www.hks.harvard.edu/m-rcbg/heep/papers/HEEP\%20Discussion\%2012.pdf. 


\section{Appendices}

Appendix Table 1. Matching of Philippine Input-Output sectors with GTAP sectors.

\begin{tabular}{|c|c|c|c|}
\hline \multicolumn{2}{|r|}{ Philippine Input-Output Table } & \multicolumn{2}{|r|}{ GTAP Concordance } \\
\hline No. & Original IO Sector Name & Code & Sectors \\
\hline 1 & Palay & PDR & Paddy rice \\
\hline 2 & Corn & GRO & Cereal grains nec \\
\hline 3 & Coconut including copra making in the farm & OSD & Oil seeds \\
\hline 4 & Banana & V_F & Vegetables, fruit, nuts \\
\hline 5 & Sugarcane including muscovado sugar done in the farm & $\mathrm{C}_{-} \mathrm{B}$ & Sugar cane, sugar beet \\
\hline 6 & Leafy and stem vegetables & $\mathrm{V}_{-} \mathrm{F}$ & Vegetables, fruit, nuts \\
\hline 7 & Other vegetables, tubers and root crops & OCR & Crops nec \\
\hline 8 & Pineapple & V_F & Vegetables, fruit, nuts \\
\hline 9 & Mango & $\mathrm{V}_{-}^{-} \mathrm{F}$ & Vegetables, fruit, nuts \\
\hline 10 & Citrus fruits & $\mathrm{V}_{-} \mathrm{F}$ & Vegetables, fruit, nuts \\
\hline 11 & Other fruits and nuts & V_F & Vegetables, fruit, nuts \\
\hline 12 & Tobacco & OCR & Crops nec \\
\hline 13 & Abaca & PFB & Plant-based fibers \\
\hline 14 & Other fibercrops & PFB & Plant-based fibers \\
\hline 15 & Coffee & OCR & Crops nec \\
\hline 16 & Cacao & OCR & Crops nec \\
\hline 17 & Rubber & OCR & Crops nec \\
\hline 18 & Ornamental/Horticultural plants/Herbal plants & OCR & Crops nec \\
\hline & Other agricultural crops (wheat, milled oats, cereal crops, spice & & Wheat \\
\hline 19 & crops and construction related crops) & WHT & \\
\hline 20 & $\begin{array}{l}\text { Agricultural services (irrigation and pesticides, artificial } \\
\text { insemination, n.e.c.) }\end{array}$ & FRS & Forestry \\
\hline 21 & Hog & OAP & Animal products nec \\
\hline 22 & Cattle & CTL & $\begin{array}{l}\text { Bovine cattle, sheep and goats, } \\
\text { horses }\end{array}$ \\
\hline 23 & Carabao & CTL & $\begin{array}{l}\text { Bovine cattle, sheep and goats, } \\
\text { horses }\end{array}$ \\
\hline 24 & Other livestock including dairy production & RMK & Raw milk \\
\hline 25 & Chicken & OAP & Animal products nec \\
\hline 26 & Other poultry & OAP & Animal products nec \\
\hline 27 & Egg production & OAP & Animal products nec \\
\hline 28 & Ocean fishing (including fish corals) & FSH & Fishing \\
\hline 29 & Inland and coastal fishing & FSH & Fishing \\
\hline 30 & Pearl culture and pearl shell gathering & FSH & Fishing \\
\hline 31 & Seaweeds & FSH & Fishing \\
\hline 32 & $\begin{array}{l}\text { Shirmp, prawns and other aquaculture (including marine } \\
\text { culture) }\end{array}$ & FSH & Fishing \\
\hline 33 & Forestry & FRS & Forestry \\
\hline 34 & Copper mining & OMN & Minerals nec \\
\hline 35 & Gold mining & OMN & Minerals nec \\
\hline 36 & Chromite mining & OMN & Minerals nec \\
\hline 37 & Nickel mining & OMN & Minerals nec \\
\hline 38 & Other metallic mining (including silver mining) & OMN & Minerals nec \\
\hline 39 & Stone quarrying, clay and sand pits & $\mathrm{OMN}$ & Minerals nec \\
\hline 40 & Coal mining & $\mathrm{COA}$ & Coal \\
\hline 41 & Crude oil and natural gas & OIL & Oil \\
\hline 42 & Other non-metallic mining (including salt mining) & OMN & Minerals nec \\
\hline 43 & Slaughtering and meat packing & CMT & Bovine meat products \\
\hline 44 & Meat and meat products processing & OMT & Meat products nec \\
\hline 45 & Milk processing & MIL & Dairy products \\
\hline 46 & Butter and cheese manufacturing & MIL & Dairy products \\
\hline 47 & Ice cream, sherbets and other flavored ices & MIL & Dairy products \\
\hline 48 & Other dairy products & MIL & Dairy products \\
\hline
\end{tabular}


49 Canning and preserving of fruits and vegetables

50 Fish canning

Fish drying, smoking and manufacturing of other seafood

51 products

52 Production of crude coconut oil, copra cake and meal Other crude vegetable oil, fish and other marine oils and fats

53 (except coconut oil)

54 Manufacture of refined coconut oil and vegetable oil

55 Rice and corn milling

56 Flour, cassava and other grains milling

57 Manufacture of bakery products except noodles

58 Noodles manufacturing

59 Sugar milling and refining

Manufacture of cocoa, chocolate and sugar confectionery

60 products

61 Manufacture of desiccated coconut

62 Manufacture of ice, except dry ice

63 Coffee roasting and processing

64 Manufacture of animal feeds

65 Manufacture of starch and starch products

Manufacture of flavoring extracts, mayonnaise and food

66 coloring products

67 Miscellaneous food products

68 Alcoholic liquors and wine

69 Malt liquors and malt

70 Softdrinks and carbonated water

71 Bottling of Mineral Water

72 Cigarette manufacturing

73 Cigar, chewing and smoking tobacco

74 Tobacco leaf flue-curing and redrying

75 Textile, spinning, weaving, texturizing and finishing

76 Fabric knitting mills

77 Hosiery, underwear and outerwear (knitted)

78 Manufacture of made-up textile goods except wearing apparel

79 Manufacture of carpets and rugs

80 Cordage, rope, twine and net manufacturing

81 Manufacture of articles made of native materials

Manufacture of artificial leather and impregnated and coated

82 fabrics

Manufacture of fiber batting, padding, upholstery fillings

including coir, linoleum and other hard surfaced floor

83 coverings

84 Custom tailoring and dressmaking shops

85 Manufacture of ready-made clothing

86 Embroidery establishments

87 Manufacture of other wearing apparel except footwear

88 Manufacture of leather footwear and footwear parts

89 Sawmills and planing of wood

90 Manufacture of veneer and plywood

91 Manufacture of hardboard and particle board

92 Wood drying and preserving plants

93 Millwork plants

Manufacture of wooden and cane containers and small cane

94 wares

95 Manufacture of wood carvings

96 Manufacture of misc wood, cork and cane products

Manufacture and repair of wooden furniture including

97 upholstery

98 Manufacture and repair of rattan furniture including upholstery

99 Manufacture and repair of other furnitures and fixtures, n.e.c.
OFD Food products nec

OFD Food products nec

Food products nec

OFD

VOL Vegetable oils and fats

Vegetable oils and fats

VOL

VOL Vegetable oils and fats

PCR Processed rice

OFD Food products nec

OFD Food products nec

OFD Food products nec

SGR Sugar

OFD

Food products nec

OFD Food products nec

OFD Food products nec

OFD Food products nec

OFD Food products nec

OFD Food products nec

Food products nec

OFD

OFD Food products nec

B_T Beverages and tobacco products

B_T Beverages and tobacco products

B_T Beverages and tobacco products

B_T Beverages and tobacco products

B_T Beverages and tobacco products

B_T Beverages and tobacco products

B_T Beverages and tobacco products

TEX Textiles

TEX Textiles

WAP Wearing apparel

WAP Wearing apparel

TEX Textiles

TEX Textiles

OME Machinery and equipment nec

Leather products

LEA

Chemical, rubber, plastic products

CRP

WAP Wearing apparel

WAP Wearing apparel

TEX Textiles

WAP Wearing apparel

LEA Leather products

LUM Wood products

LUM Wood products

LUM Wood products

LUM Wood products

LUM Wood products

Wood products

LUM

LUM Wood products

LUM Wood products

Manufactures nec

OMF

OMF Manufactures nec

OMF Manufactures nec 
100 Manufacture of pulp, paper and paperboard

101 Manufacture of paper and paperboard containers

102 Manufacture of articles of paper and paperboard

103 Newspapers and periodicals

104 Printing and publishing of books and pamphlets

105 Commercial and job printing and other allied industries

106 Tanneries and leather finishing

Manufacture of products of leather and leather substitutes

107 except footwear and wearing apparel

108 Rubber tire and tube manufacturing

109 Manufacture of rubber footwear

110 Manufacture of other rubber products, n.e.c.

111 Manufacture of basic industrial chemicals

112 Manufacture of fertilizers

Manufacture of synthetic resins, plastic materials and other

113 man-made fiber except glass

114 Manufacture of pesticides, insecticides, etc.

115 Manufacture of paints, varnishes and lacquers

116 Manufacture of drugs and medicines

117 Manufacture of soap and detergents

Manufacture of perfumes, cosmetics and other toilet

118 preparations

119 Manufacture of miscellaneous chemical products

Manufacture of plastic furniture, plastic footwear and other

120 fabricated plastic products

121 Petroleum refineries including LPG

Manufacture of asphalt, lubricants and miscellaneous products

122 of petroleum and coal

123 Manufacture of pottery,china and earthenwares

124 Manufacture of flat glass

125 Manufacture of glass container

126 Manufacture of other glass and glass products

127 Cement manufacture

128 Manufacture of structural clay products

129 Manufacture of structural concrete products

130 Manufacture of other non-metallic mineral products, n.e.c.

Blast furnace and steel making furnace, steel works and rolling

131 mills

132 Iron and steel foundries

Non-ferrous smelting and refining plants, rolling, drawing and

133 extrusion mills

134 Non-ferrous foundries

135 Cutlery, handtools, general hardware

136 Structural metal products

137 Manufacture of metal containers

138 Metal stamping, coating, engraving mills

139 Manufacture of wire nails

Manufacture of other fabricated wire and cable products except

140 insulated wire and cable

141 Manufacture of non-electric lighting and heating fixtures Manufacture of fabricated metal products except machinery

142 and equipment

143 Manufacture of agricultural machinery and equipment

144 Manufacture of metal and wood-working machinery Manufacture of engines and turbines, except for transport

145 equipment and special industrial machinery and equipment Manufacture, assembly and repair of office, computing and

146 accounting machs

Manufacture of pumps, compressors, blowers and

147 airconditioners
PPP

PPP

PPP

PPP

PPP

PPP

LEA

LEA

CRP

LEA

CRP

CRP

CRP

CRP

CRP

CRP

CRP

CRP

CRP

CRP

CRP

P_C

P_C

NMM

NMM

NMM

NMM

NMM

NMM

NMM

NMM

I S

I_S

NFM

NFM

FMP

FMP

FMP

FMP

FMP

FMP

FMP

FMP

OME

OME

OME

ELE

OME
Paper products, publishing

Paper products, publishing

Paper products, publishing

Paper products, publishing

Paper products, publishing

Paper products, publishing

Leather products

Leather products

Leather products

Chemical, rubber, plastic products

Chemical, rubber, plastic products

Chemical, rubber, plastic products

Chemical, rubber, plastic products

Chemical, rubber, plastic products

Chemical, rubber, plastic products

Chemical, rubber, plastic products

Chemical, rubber, plastic products

Chemical, rubber, plastic products

Chemical, rubber, plastic products

Chemical, rubber, plastic products

Petroleum, coal products

Petroleum, coal products

Mineral products nec

Mineral products nec

Mineral products nec

Mineral products nec

Mineral products nec

Mineral products nec

Mineral products nec

Mineral products nec

Ferrous metals

Ferrous metals

Metals nec

Metals nec

Metal products

Metal products

Metal products

Metal products

Metal products

Metal products

Metal products

Metal products

Machinery and equipment nec

Machinery and equipment nec

Machinery and equipment nec

Electronic equipment

Machinery and equipment nec 
Machine shops and manufacture of non-electrical machinery

148 and equipment, n.e.c.

149 Manufacture of electrical, industrial machinery and apparatus Manufacture of radio and TV receiving sets, sound recording

150 and reproducing equipment including records and tapes

151 Manufacture of communication and detection equipment Manufacture of parts and supplies for radio, TV and

152 communication

153 Manufacture of appliances and housewares

154 Manufacture of semi- conductor devices

Manufacture of primary cells and batteries and electric

155 accumulators

156 Insulated wires and cables

Manufacture of current-carrying wiring devices, conduits and

157 fittings

Manufacture of electrical lamps, fluorescent tubes and other

158 electrical apparatus and supplies, n.e.c.

159 Shipyards and boatyards

160 Manufacture and assembly of motor vehicles

161 Rebuilding and major alteration of motor vehicles

162 Manufacture of motor vehicles parts and accessories

163 Manufacture, assembly of motorcycles and bicycles Manufacture, assembly, rebuilding and major alteration of railroad equipment, aircraft, and animal and hand-drawn

164 vehicle

Manufacture of professional, scientific measuring and

165 controlling equipment

166 Manufacture of photographic and optical instruments

167 Manufacture of watches and clocks

Manufacture and repair of furniture and fixtures, made

168 primarily of metal

169 Manufacture of jewelry and related articles

170 Manufacture of musical instruments

171 Manufacture of sporting and athletic goods

Manufacture of surgical, dental, medical and orthopedic

172 supplies

173 Manufacture of opthalmic goods

174 Manufacture of toys and dolls except rubber and plastic toys

175 Manufacture of stationers', artists' and office supplies

176 Miscellaneous manufacturing

177 Construction

178 Electricity

179 Steam

180 Water

181 Railway transport

182 Bus line operation

183 Public utility cars and taxicab operation

Jeepney, tricycles (motorized and non-motorized) and other

184 road transport

185 Tourist buses and cars including chartered and rent-a-car

186 Road freight transport

187 Sea and coastal water transport

188 Inland water transport (including renting of ship with operator)

189 Supporting services to transport

190 Air transport

191 Tour and travel agencies

Activities of other transport agencies (including custom

192 brokerage, n.e.c)

193 Storage and warehousing

194 Postal and courier activities
OME

OME Machinery and equipment nec Electronic equipment

ELE

ELE Electronic equipment Electronic equipment

ELE

ELE

ELE

OME

OME Machinery and equipment nec Machinery and equipment nec

OME

OME

OTN Transport equipment nec

MVH Motor vehicles and parts

MVH Motor vehicles and parts

MVH Motor vehicles and parts

OTN Transport equipment nec

Transport equipment nec

OTN

OME

OME Machinery and equipment nec

OME Machinery and equipment nec Manufactures nec

OMF

OMF

$\mathrm{OMF}$

$\mathrm{OMF}$

OME

OME

$\mathrm{OMF}$

OMF

OMF

CNS

ELY

GDT

WTR

OTP

OTP

OTP

Manufactures nec

Manufactures nec

Manufactures nec

Machinery and equipment nec

OTP

OTP

OTP

WTP

WTP

OTP

ATP

OTP

OTP

OTP

CMN
Machinery and equipment nec

Manufactures nec

Manufactures nec

Manufactures nec

Construction

Electricity

Gas manufacture, distribute

Water

Transport nec

Transport nec

Transport nec

Transport nec

ansport nec

Water transport

Water transport

Transport nec

ir transport

Transport nec

Transport nec

Transport nec

Communication 
195 Telephone service includes telegraphs

196 Wireless telecommunications

197 Telecommunication services, n.e.c

198 Wholesale and retail trade

199 Repairs of motor vehicles and personal and household goods

200 Banking

Investment, financing and other non-banking services except

201 pawnshops

202 Pawnshops

203 Life insurance

204 Non-life and other insurance activities

Real estate activities with own or leased property and contract

205 basis

206 Ownership of dwellings

207 Public Education Services

208 Public Health and Welfare Services

209 Public Administration and Defense

210 Private education services

211 Private medical, dental and other health services

Other hospital activities and medical and dental practices,

212 including veterinary services

213 Legal activities

214 Accounting, bookkeeping and auditing activities; tax cons

215 Architectural and engineering activities

216 Advertising activities

217 Renting of equipments

218 Business and management cons activities

219 Labor recruitment and provision of personnel

220 Investigation and security activities

221 Miscellaneous business activities, n.e.c.

222 Other business services

223 Hotels and motels

224 Other short-stay accommodation, n.e.c.

225 Restaurants, bars, canteens, other eating, drinking places

226 Computer hardware cons

227 Computer software cons including computer supply

228 Other computer related activities

Maintenance and repair of office, accounting and computing

229 machinery

230 Photographic activities

Call/Contact centers, Business Processing outsourcing and

231 other IT-based services

232 Social Work

233 Sewage and refuse disposal, sanitation and similar activities

234 Motion picture and video production and distribution

235 Motion picture projection

236 Radio and television activities

237 Other recreational and cultural services

238 Washing and drycleaning of clothing and textile

239 Hairdressing and other beauty treatment

240 Other personal services, n.e.c.
CMN Communication

CMN Communication

CMN Communication

TRD Trade

TRD Trade

OFI Financial services nec

Financial services nec

OFI

OFI Financial services nec

ISR Insurance

ISR Insurance

Business services nec

OBS

DWE Dwellings

Public Administration, Defense,

OSG Education, Health

Public Administration, Defense,

OSG Education, Health

Public Administration, Defense,

OSG Education, Health

Public Administration, Defense,

OSG Education, Health

Public Administration, Defense,

OSG Education, Health

Public Administration, Defense,

OSG Education, Health

ROS Recreational, other services

ROS Recreational, other services

ROS Recreational, other services

ROS Recreational, other services

ROS Recreational, other services

ROS Recreational, other services

ROS Recreational, other services

ROS Recreational, other services

OBS Business services nec

OBS Business services nec

TRD Trade

TRD Trade

TRD Trade

ROS Recreational, other services

ROS Recreational, other services

ROS Recreational, other services

Recreational, other services

ROS

OBS Business services nec

Business services nec

OBS

OSG Education, Health

Public Administration, Defense,

OSG Education, Health

ROS Recreational, other services

ROS Recreational, other services

ROS Recreational, other services

ROS Recreational, other services

ROS Recreational, other services

ROS Recreational, other services

ROS Recreational, other services

Note: GTAP has 57 aggregated sectors and this was disaggregated into 240 sectors to match with Philippine Input-Output Table for year 2000. Source: Corong (2008) 
Appendix Table 2. $\mathrm{CO}_{2}$ emission intensities of different economic sectors.

\begin{tabular}{|c|c|c|c|c|c|}
\hline & Sectors & $\begin{array}{l}\left(\mathrm{g} \mathrm{CO}_{2} /\right. \\
\text { peso) }\end{array}$ & & Sectors & $\begin{array}{c}\left(\mathrm{g} \mathrm{CO}_{2} /\right. \\
\text { peso) }\end{array}$ \\
\hline 001 & Palay & 2.5977 & 041 & Crude oil and natural gas & 15.5915 \\
\hline 002 & Corn & 2.0624 & 042 & Other non-metallic mining & 8.7536 \\
\hline 003 & Coconut including copra & 1.2173 & 043 & Slaughtering and meat packing & 6.0051 \\
\hline 004 & Banana & 4.4112 & 044 & Meat and meat products & 11.6183 \\
\hline 005 & Sugarcane & 6.8952 & 045 & Milk processing & 10.9493 \\
\hline 006 & Leafy and stem vegetables & 2.6091 & 046 & Butter and cheese manufacturing & 14.8264 \\
\hline 007 & Tubers and root crops & 1.1528 & 047 & Ice cream, sherbets and others & 16.0977 \\
\hline 008 & Pineapple & 2.2363 & 048 & Other dairy products & 7.1311 \\
\hline 009 & Mango & 2.2189 & 049 & Preserving of fruits \& vegetables & 8.5577 \\
\hline 010 & Citrus fruits & 3.3846 & 050 & Fish canning & 12.8933 \\
\hline 011 & Other fruits and nuts & 1.7225 & 051 & Fish drying and other seafoods & 12.2518 \\
\hline 012 & Tobacco & 2.6526 & 052 & Production of crude coconut oil & 5.9048 \\
\hline 013 & Abaca & 8.8263 & 053 & Other crude veg oil \& marine oil & 4.8744 \\
\hline 014 & Other fibercrops & 3.5360 & 054 & Refined coconut $\&$ vegetable oil & 9.8145 \\
\hline 015 & Coffee & 5.6261 & 055 & Rice and corn milling & 3.3026 \\
\hline 016 & Cacao & 7.3321 & 056 & Flour, cassava and other grains & 6.2811 \\
\hline 017 & Rubber & 8.2860 & 057 & Manufacture of bakery products & 14.0443 \\
\hline 018 & Horticultural or herbal plants & 7.3167 & 058 & Noodles manufacturing & 10.8511 \\
\hline 019 & Other agricultural crops & 0.7242 & 059 & Sugar milling and refining & 20.9218 \\
\hline 020 & Agricultural services & 4.1135 & 060 & Cocoa, chocolate and sugar & 13.3603 \\
\hline 021 & Hog & 4.5306 & 061 & Desiccated coconut & 8.4988 \\
\hline 022 & Cattle & 2.7612 & 062 & Manufacture of ice except dry ice & 53.6820 \\
\hline 023 & Carabao & 3.3390 & 063 & Coffee roasting and processing & 12.4155 \\
\hline 024 & Other livestock including dairy & 3.8293 & 064 & Manufacture of animal feeds & 7.9322 \\
\hline 025 & Chicken & 8.8800 & 065 & Starch and starch products & 7.9827 \\
\hline 026 & Other poultry & 3.8649 & 066 & Flavoring extracts, mayonnaise & 13.4184 \\
\hline 027 & Egg production & 9.6266 & 067 & Miscellaneous food products & 8.9454 \\
\hline 028 & Ocean fishing & 11.1417 & 068 & Alcoholic liquors and wine & 15.4253 \\
\hline 029 & Inland and coastal fishing & 10.1459 & 069 & Malt liquors and malt & 10.7830 \\
\hline 030 & Pearl culture & 14.5675 & 070 & Softdrinks and carbonated water & 19.4368 \\
\hline 031 & Seaweeds & 10.5784 & 071 & Bottling of Mineral Water & 13.9163 \\
\hline 032 & Shrimps, prawns and others & 9.9112 & 072 & Cigarette manufacturing & 9.0450 \\
\hline 033 & Forestry & 3.2852 & 073 & Cigar, chewing, smoking tobacco & 11.2867 \\
\hline 034 & Copper mining & 8.0165 & 074 & Tobacco leaf and redrying & 8.6577 \\
\hline 035 & Gold mining & 24.1232 & 075 & Textile, spinning, weaving & 11.6441 \\
\hline 036 & Chromite mining & 158.8611 & 076 & Fabric knitting mills & 6.5027 \\
\hline 037 & Nickel mining & 15.6035 & 077 & Hosiery, underwear and others & 10.4487 \\
\hline 038 & Other metallic mining & 5.0955 & 078 & Made-up textile goods & 8.7047 \\
\hline 039 & Stone quarrying, clay and sand & 8.8739 & 079 & Manufacture of carpets and rugs & 12.1250 \\
\hline 040 & Coal mining & 77.9721 & 080 & Cordage, rope, twine and net & 11.8642 \\
\hline
\end{tabular}


continuation Appendix Table 2. $\mathrm{CO}_{2}$ emission intensities of different economic sectors.

\begin{tabular}{|c|c|c|c|c|c|}
\hline & Sectors & $\begin{array}{l}\left(\mathrm{g} \mathrm{CO}_{2} /\right. \\
\text { peso })\end{array}$ & & Sectors & $\begin{array}{l}\left(\mathrm{g} \mathrm{CO}_{2} /\right. \\
\text { peso })\end{array}$ \\
\hline 081 & Articles made of native materials & 12.5916 & 121 & Petroleum refineries incl LPG & 17.8117 \\
\hline 082 & Artificial leather \& coated fabric & 6.3106 & 122 & Asphalt, lubricants and misc prod & 14.1770 \\
\hline 083 & Upholstery fillings \& coverings & 18.0657 & 123 & Pottery,china and earthen wares & 53.4887 \\
\hline 084 & Tailoring and dressmaking shops & 6.0728 & 124 & Manufacture of flat glass & 50.0564 \\
\hline 085 & Ready-made clothing & 9.8198 & 125 & Manufacture of glass container & 63.8897 \\
\hline 086 & Embroidery establishments & 9.9360 & 126 & Other glass and glass products & 72.0594 \\
\hline 087 & Other wearing apparel & 7.7439 & 127 & Cement manufacture & 72.5321 \\
\hline 088 & Leather footwear \& footwear part & 9.1598 & 128 & Structural clay products & 57.9100 \\
\hline 089 & Sawmills and planing of wood & 6.2090 & 129 & Structural concrete products & 78.4560 \\
\hline 090 & Veneer \& plywood & 8.5653 & 130 & Other non-metallic mineral prod & 76.0676 \\
\hline 091 & Hardboard and particle board & 16.6654 & 131 & Blast furnace and steel making & 30.1788 \\
\hline 092 & Wood drying \& preserving plants & 8.2833 & 132 & Iron and steel foundries & 15.8577 \\
\hline 093 & Millwork plants & 7.2849 & 133 & Non-ferrous smelting \& refining & 14.7005 \\
\hline 094 & Wooden and cane containers & 7.4057 & 134 & Non-ferrous foundries & 13.9779 \\
\hline 095 & Manufacture of wood carvings & 4.4727 & 135 & Cutlery, handtools, gen hat & 12.2750 \\
\hline 096 & Cork, cane products other wood & 5.9942 & 136 & Structural metal products & 24.3169 \\
\hline 097 & wooden furniture incl upholstery & 7.6400 & 137 & Manufacture of metal conta & 18.7588 \\
\hline 098 & Rattan furniture incl upholstery & 11.2440 & 138 & Metal stamping, engraving & 28.4259 \\
\hline 099 & Other furniture and fixtures, nec & 7.5057 & 139 & Manufacture of wire nails & 26.7619 \\
\hline 100 & Pulp, paper and paperboard & 19.4567 & 140 & Other fabricated wire and cable & 29.2278 \\
\hline 101 & Paper and paperboard containers & 21.5235 & 141 & Non-electric lighting \& & 12.1978 \\
\hline 102 & Articles of paper and paperboard & 20.7168 & 142 & Fabricated metal products & 15.6944 \\
\hline 103 & Newspapers and periodicals & 15.2149 & 143 & Agricultural machinery \& equip & 10.0377 \\
\hline 104 & Printing and publishing of boo & 12.1005 & 144 & Metal \&woodworking machinery & 1.5920 \\
\hline 105 & Commercial and job printing & 26.1859 & 145 & Engines and turbines & 7.8843 \\
\hline 106 & Tanneries and leather finishing & 7.1854 & 146 & Assembly and repair of office & 6.6907 \\
\hline 107 & Leather and leather substitutes & 7.3237 & 147 & Pumps, compressors, and aircon & 3.8120 \\
\hline 108 & Rubber tire \& tube manufacturing & 10.2385 & 148 & Nonelectrical machinery \& equip & 10.6587 \\
\hline 109 & Manufacture of rubber footwear & 10.3609 & 149 & Electrical, industrial machinery & 4.9998 \\
\hline 110 & Manufacture of other rubber prod & 8.4112 & 150 & Radio and TV receiving sets & 2.5782 \\
\hline 111 & Basic industrial chemicals & 8.5990 & 151 & Communication, detection equip & 11.4675 \\
\hline 112 & Manufacture of fertilizers & 7.8043 & 152 & Parts \& supplies for radio, TV & 2.6046 \\
\hline 113 & Synthetic resins, plastic materials & 6.4265 & 153 & Appliances and house wares & 5.6070 \\
\hline 114 & Pesticides, insecticides, etc & 8.9188 & 154 & Semi- conductor devices & 3.0416 \\
\hline 115 & Paints, varnishes and lacquers & 8.8564 & 155 & Primary cells and batteries & 22.0252 \\
\hline 116 & Drugs and medicines & 9.5862 & 156 & Insulated wires and cables & 11.6584 \\
\hline 117 & Soap and detergents & 7.8468 & 157 & Conduits and fittings & 9.5898 \\
\hline 118 & Perfumes, cosmetics and other & 10.7909 & 158 & Electrical lamps, fluorescent & 12.5013 \\
\hline 119 & Miscellaneous chemical products & 6.3937 & 159 & Shipyards and boatyards & 14.5340 \\
\hline 120 & Plastic furniture, plastic footwear & 9.7269 & 160 & Motor vehicles & 9.9922 \\
\hline
\end{tabular}


continuation Appendix Table 2. $\mathrm{CO}_{2}$ emission intensities of different economic sectors.

\begin{tabular}{|c|c|c|c|c|c|}
\hline & Sectors & $\begin{array}{l}\left(\mathrm{g} \mathrm{CO}_{2} /\right. \\
\text { peso })\end{array}$ & & Sectors & $\begin{array}{c}\left(\mathrm{g} \mathrm{CO}_{2} /\right. \\
\text { peso })\end{array}$ \\
\hline 161 & Alteration of motor vehicles & 9.6812 & 201 & Investment, financing & 5.9047 \\
\hline 162 & motor vehicles parts, accessories & 4.7272 & 202 & Pawnshops & 8.0381 \\
\hline 163 & Motorcycles and bicycles & 6.1819 & 203 & Life insurance & 7.0236 \\
\hline 164 & Railroad equipment, aircraft & 3.8601 & 204 & Non-life and other insurance & 6.7358 \\
\hline 165 & sci measuring \&controlling equip & 4.5386 & 205 & Real estate activities with own & 7.8993 \\
\hline 166 & Photographic and optical ins & 7.2614 & 206 & Ownership of dwellings & 1.4512 \\
\hline 167 & Watches and clocks & 10.4594 & 207 & Public Education Services & 5.5403 \\
\hline 168 & Furniture and fixtures (metal) & 14.1466 & 208 & Public Health, Welfare Services & 10.4251 \\
\hline 169 & Jewelry and related articles & 8.6943 & 209 & Public Administration, Defense & 10.2024 \\
\hline 170 & Musical instruments & 4.0565 & 210 & Private education services & 15.0456 \\
\hline 171 & Sporting and athletic goods & 11.1562 & 211 & Private medical, dental services & 10.8915 \\
\hline 172 & Surgical, dental, medical supplies & 8.4373 & 212 & Other hospital act, veterinary & 11.3958 \\
\hline 173 & Manufacture of opthalmic goods & 18.5782 & 213 & Legal activities & 9.8341 \\
\hline 174 & Manufacture of toys and dolls & 6.5015 & 214 & Accounting, bookkeeping & 6.6373 \\
\hline 175 & Artists' and office supplies & 7.5571 & 215 & Architectural and engineering act & 9.1896 \\
\hline 176 & Miscellaneous manufacturing & 5.6357 & 216 & Advertising activities & 5.2220 \\
\hline 177 & Construction & 18.7418 & 217 & Renting of equipments & 8.0504 \\
\hline 178 & Electricity & 157.2655 & 218 & Business \& mgt consultancy & 6.7033 \\
\hline 179 & Steam & 27.1428 & 219 & Labor recruitment & 7.4762 \\
\hline 180 & Water & 67.4639 & 220 & Investigation,security activit & 7.0653 \\
\hline 181 & Railway transport & 63.1313 & 221 & Miscellaneous business act & 12.3407 \\
\hline 182 & Bus line operation & 51.3768 & 222 & Other business services & 15.0574 \\
\hline 183 & Public utility cars, taxi operation & 52.6268 & 223 & Hotels and motels & 7.0647 \\
\hline 184 & Jeepney, tricycles, other transport & 52.1624 & 224 & Other short-stay accommodation & 7.5546 \\
\hline 185 & Tourist buses and cars & 51.1772 & 225 & Restaurants, bars, canteens & 8.2660 \\
\hline 186 & Road freight transport & 52.4432 & 226 & Computer hardware consultancy & 22.1183 \\
\hline 187 & Sea and coastal water transport & 14.7302 & 227 & Computer software consultancy & 10.9708 \\
\hline 188 & Inland water transport & 20.6338 & 228 & Other computer related activities & 7.4959 \\
\hline 189 & Supporting services to transport & 49.5953 & 229 & Maintenance of office machinery & 5.3453 \\
\hline 190 & Air transport & 71.1750 & 230 & Photographic activities & 10.7927 \\
\hline 191 & Tour and travel agencies & 57.2530 & 231 & Call/Contact centers, other IT & 15.4255 \\
\hline 192 & Other transport agencies & 55.2023 & 232 & Social Work & 14.9750 \\
\hline 193 & Storage and warehousing & 50.2993 & 233 & Sewage and refuse disposal & 9.4491 \\
\hline 194 & Postal and courier activities & 6.0506 & 234 & Motion picture and video prod & 4.2822 \\
\hline 195 & Telephone service, telegraphs & 4.3237 & 235 & Motion picture projection & 27.4294 \\
\hline 196 & Wireless telecommunications & 4.3493 & 236 & Radio and television activities & 8.6124 \\
\hline 197 & Telecommunication services & 5.1666 & 237 & Other recreational \& cultural serv & 7.1663 \\
\hline 198 & Wholesale and retail trade & 8.7348 & 238 & Washing and dry cleaning & 19.9805 \\
\hline 199 & Repair of vehicles \& other goods & 19.5959 & 239 & Hairdressing, other beauty treat & 16.3685 \\
\hline 200 & Banking & 8.6518 & 240 & Other personal services, n.e.c. & 6.9441 \\
\hline
\end{tabular}


Appendix Table 3. Matching of household consumption items with IO sectors.

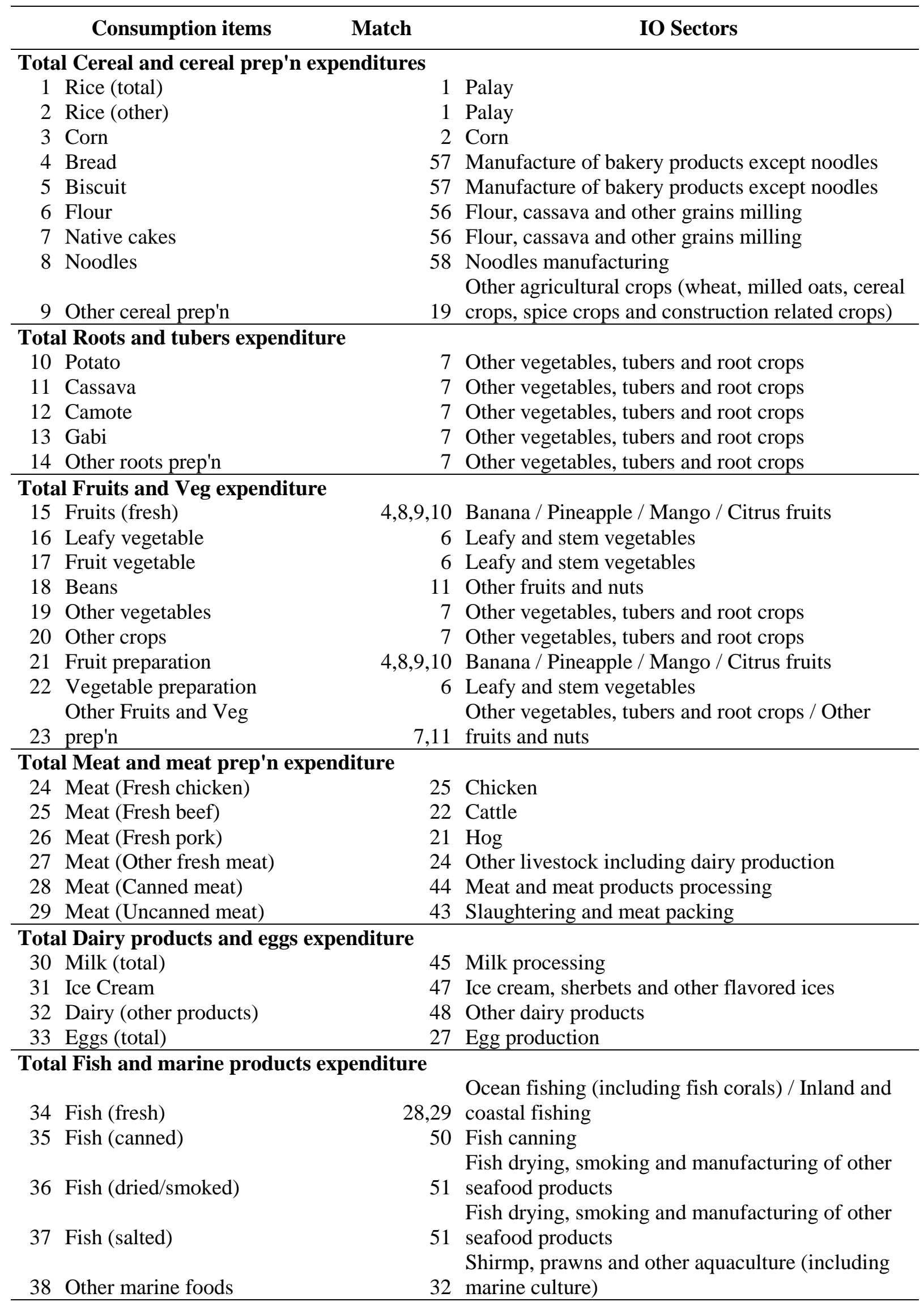




\begin{tabular}{|c|c|c|}
\hline \multicolumn{3}{|c|}{ Total Coffee, cocoa and tea expenditure } \\
\hline 39 Coffee (processed) & 63 & Coffee roasting and processing \\
\hline 40 Coffee (beans) & 15 & Coffee \\
\hline 41 Cocoa (beans) & 16 & Cacao \\
\hline 42 Tea & 67 & Miscellaneous food products \\
\hline \multicolumn{3}{|c|}{ Total Non-alcoholic beverages expenditure } \\
\hline 43 Soft drinks & 70 & Softdrinks and carbonated water \\
\hline 44 Fruit juice & $4,8,9,10$ & Banana / Pineapple / Mango / Citrus fruits \\
\hline Other non-alcholic & & \\
\hline 45 beverages & 70 & Softdrinks and carbonated water \\
\hline 46 Soda drinks & 70 & Softdrinks and carbonated water \\
\hline 47 Bottled water & 71 & Bottling of Mineral Water \\
\hline \multicolumn{3}{|l|}{ Total Food N.E.C expenditure } \\
\hline 48 Sugar & 59 & Sugar milling and refining \\
\hline 49 Sugar products & 59 & $\begin{array}{l}\text { Sugar milling and refining } \\
\text { Manufacture of refined coconut oil and vegetable }\end{array}$ \\
\hline 50 Cooking oil & 54 & $\begin{array}{l}\text { oil } \\
\text { Other crude vegetable oil, fish and other marine }\end{array}$ \\
\hline 51 Margarine & 53 & oils and fats (except coconut oil) \\
\hline 52 Sauces & 67 & Miscellaneous food products \\
\hline 53 Salt & 42 & Other non-metallic mining (including salt mining) \\
\hline Other spices and & & Manufacture of flavoring extracts, mayonnaise and \\
\hline 54 seasoning & 66 & food coloring products \\
\hline Prepared meals bought & & Restaurants, bars, canteens andother eating and \\
\hline 55 outside & 225 & drinking places \\
\hline 56 Other food n.e.c & 67 & Miscellaneous food products \\
\hline \multicolumn{3}{|c|}{ Total Alcoholic beverages expenditure } \\
\hline 57 Beer & 69 & Malt liquors and malt \\
\hline 58 Wine & 68 & Alcoholic liquors and wine \\
\hline 59 Liquor & 68,69 & Alcoholic liquors and wine / Malt liquors and malt \\
\hline 60 Tobacco (cigarette) & 72 & Cigarette manufacturing \\
\hline 61 Tobacco (cigar) & 73 & Cigar, chewing and smoking tobacco \\
\hline 62 Tobacco (others) & 12 & Tobacco \\
\hline \multicolumn{3}{|c|}{ Total fuel, light and water expenditure } \\
\hline 63 Charcoal & 33 & Forestry \\
\hline 64 Firewood & 33 & Forestry \\
\hline 65 LPG & 121 & Petroleum refineries including LPG \\
\hline 66 Petroleum products & 121 & Petroleum refineries including LPG \\
\hline 67 Electricity & 178 & Electricity \\
\hline 68 Water & 180 & Water \\
\hline 69 Other fuel, light and water & 179 & Steam \\
\hline
\end{tabular}

Total Transport and Comm. expenditure

70 Transport (land fare)

71 Transport (air fare)

72 Transport (water fare) Transport

73 (Gasoline/Diesel)

74 Transport (Maint./ Repair)

75 Transport (Driver's salary) Transport (Personal,
Railway / Bus / Public utility cars and taxi / $181,182,183$, Jeepney, tricycle and other road transport / Road $184,185,186$ freight transport

190 Air transport Sea and coastal water transport / Inland water

187,188 transport

41 Crude oil and natural gas

Repairs of motor vehicles and personal and 199 household goods 
77 Telephone bills

78 Postage stamps

79 Telegrams

80 Other transpo and comm.

81 Phone cards
195 Telephone service includes telegraphs

194 Postal and courier activities

197 Telecommunication services, n.e.c Activities of other transport agencies / Other

192, 197 telecommunications services Wireless telecommunications / Other 196, 197 telecommunication services

\section{Total Household operation expenditure}

Laundry and detergent

82 soap

83 Starch

84 Floor wax

85 Pesticide

86 Cleansing powder

87 Air freshener

88 Bulbs

Others

(matches, brooms,husks,

89 etc.)

90 Laundry services

91 Laundry services

92 Dry clean services

93 Domestic Services (total)

94 Maid/boy services

95 Gardener services

96 Other Domestic services Repairs and maintenance

97 of appliances
117 Manufacture of soap and detergents

65 Manufacture of starch and starch products

114 Manufacture of pesticides, insecticides, etc.

114 Manufacture of pesticides, insecticides, etc.

117 Manufacture of soap and detergents

114 Manufacture of pesticides, insecticides, etc. Manufacture of electrical lamps, fluorescent tubes

158 and other electrical apparatus and supplies, n.e.c.

no match

240 Other personal services, n.e.c.

240 Other personal services, n.e.c.

240 Other personal services, n.e.c.

240 Other personal services, n.e.c.

240 Other personal services, n.e.c.

240 Other personal services, n.e.c.

240 Other personal services, n.e.c.

Repairs of motor vehicles and personal and

199 household goods
Total Personal care and effects expenditure Beauty aids and toiletries

98 cash

99 Personal effects

100 Beauty parlor services

101 Barbershop Other personal care

102 services

103 Baby care

104 Adult care
Manufacture of perfumes, cosmetics and other

118 toilet preparations

Manufacture of perfumes, cosmetics and other

118 toilet preparations

239 Hairdressing and other beauty treatment

239 Hairdressing and other beauty treatment

240 Other personal services, n.e.c.

240 Other personal services, n.e.c.

240 Other personal services, n.e.c.

\section{Total Clothing, Footwear and other wear in expenditure \\ 105 Readymade men's apparel \\ Readymade women's \\ 106 apparel \\ 107 Readymade boys' apparel \\ 108 Readymade girls' apparel \\ Readymade infants' \\ 109 apparel \\ 110 Men's footwear \\ 111 Women's footwear \\ 112 Boys' footwear \\ 113 Girls' footwear \\ 85 Manufacture of ready-made clothing \\ 85 Manufacture of ready-made clothing \\ 85 Manufacture of ready-made clothing \\ 85 Manufacture of ready-made clothing \\ 85 Manufacture of ready-made clothing Leather footwear / Rubber footwear / Plastic \\ $88,109,120$ footwear \\ Leather footwear / Rubber footwear / Plastic \\ $88,109,120$ footwear \\ Leather footwear / Rubber footwear / Plastic \\ $88,109,120$ footwear \\ 88,109,120 Leather footwear / Rubber footwear / Plastic}


114 Sewing materials

115 Sewing accessories

116 Service fees on repairs

Total Educational fees expenditure

117 Tuition fees

Study allowance away

118 from home

119 Books

120 School supplies

121 Other educ supplies footwear

84 Custom tailoring and dressmaking shops

84 Custom tailoring and dressmaking shops

84 Custom tailoring and dressmaking shops

\section{Total Recreation expenditure \\ Recreational goods and \\ 122 supplies \\ 123 Musical instruments Admission tickets to \\ 124 shows Admission fees to \\ 125 cockfights and races 126 Other recreational \\ Total Medical Care expenditure \\ 127 Drugs and medicine}

128 Hospital room charges

129 Medical charges

130 Dental charges Other medical goods and

131 supplies

Other medical health

132 services

133 Contraceptives (pills, etc.)
Public education services / Private education

210,207 services

Public education services / Private education

210,207 services

104 Printing and publishing of books and pamphlets

100 Manufacture of pulp, paper and paperboard

\section{Total Non-durable furnishings expenditure}

134 Utensils and accessories Household linen and

135 furnishings Other household

136 furnishings Kitchen and laundry

137 appliances Kitchen and laundry

138 appliances in installment

139 Audio-visual equipment

Audio-visual equipment in 140 installment
170 Manufacture of musical instruments

237 Other recreational and cultural services

237 Other recreational and cultural services

237 Other recreational and cultural services

116 Manufacture of drugs and medicines Public health and welfare services / Private medical, dental and other health services / Other

208,211,212 hospital activities

Public health and welfare services / Private medical, dental and other health services / Other

208,211,212 hospital activities

Public health and welfare services / Private medical, dental and other health services / Other

208,211,212 hospital activities Manufacture of surgical, dental, medical and

172 orthopedic supplies Public health and welfare services / Private medical, dental and other health services / Other

208,211,212 hospital activities

116 Manufacture of drugs and medicines

135 Cutlery, handtools, general hardware

Manufacture of made-up textile goods except

78 wearing apparel

80 Cordage, rope, twine and net manufacturing

153 Manufacture of appliances and housewares

153 Manufacture of appliances and housewares Manufacture of radio and TV receiving sets, sound recording and reproducing equipment including records and tapes / Manufacture of parts and

150,152 supplies for radio, TV and communication Manufacture of radio and TV receiving sets, sound recording and reproducing equipment including

150,152 records and tapes / Manufacture of parts and 


\section{Furnitures}

142 Furnitures in installment

Other major appliances

143 and eqmt

Other major appliances 144 and eqmt in installment

145 Minor appliances

Minor appliances in

146 installment

147 Transport equipment

Transport equipment in

148 installment

149 Household tools Household tools in

150 installment

\section{Total Taxes}

151 Income tax

152 Real estate tax

153 Other direct taxes supplies for radio, TV and communication

Manufacture and repair of wooden furniture including upholstery / Rattan furniture / other

97,98,99 furnitures

Manufacture and repair of wooden furniture

including upholstery / Rattan furniture / other

97,98,99 furnitures

Agricultural machinery and equipment / pumps, compressors, blowers and airconditioners / electrical, industrial machinery and apparatus / communication and detection equipment /

$143,147,149$, professional, scientific measuring and controlling

$151,165,166$, equipment / watches and clocks / jewelry and

167,169 related articles

Agricultural machinery and equipment / pumps, compressors, blowers and airconditioners / electrical, industrial machinery and apparatus / communication and detection equipment /

$143,147,149$, professional, scientific measuring and controlling

$151,165,166$, equipment / watches and clocks / jewelry and

167,169 related articles

Agricultural machinery and equipment / pumps, compressors, blowers and airconditioners / electrical, industrial machinery and apparatus /

143,147,149, communication and detection equipment /

$151,165,166$, professional, scientific measuring and controlling

167,169 equipment / watches and clocks / jewelry and related articles

Agricultural machinery and equipment / pumps, compressors, blowers and airconditioners / electrical, industrial machinery and apparatus / communication and detection equipment /

$143,147,149$, professional, scientific measuring and controlling $151,165,166$, equipment / watches and clocks / jewelry and 167,169 related articles

Manufacture and assembly of motor vehicles / motor vehicles parts and accessories / assembly of

160,162,163 motorcycles and bicycles Manufacture and assembly of motor vehicles / motor vehicles parts and accessories / assembly of

160,162,163 motorcycles and bicycles

135 Cutlery, handtools, general hardware

135 Cutlery, handtools, general hardware

no match

Real estate activities with own or leased property

205 and contract basis no match

\section{Total house rental value}

154 Actual house rent

206 Ownership of dwellings

\begin{tabular}{lrl}
\hline Total House Maintenance and repairs expenditure \\
155 Carpentry materials & $89 \begin{array}{l}\text { Sawmills and planing of wood } \\
\text { Manufacture of electrical lamps, fluorescent tubes } \\
\end{array}$ \\
156 Electrical materials & 158 and other electrical apparatus and supplies, n.e.c.
\end{tabular}


157 Masonry

158 Paint, varnish, thinner, etc.

159 Plumbing materials Other construction

160 materials

161 Paid labor wages
177 Construction

115 Manufacture of paints, varnishes and lacquers

177 Construction

177 Construction

\section{Total Special Family occasion expenditure}

162 Food and refreshment

163 Alcoholic beverages Service of priests, cooks,

164 waiters, etc. Rental of space, facilities

165 and eqmt

166 Package tours Others (balloons, flowers,

167 etc.)
Softdrinks and carbonated water / Miscellaneous 67,70 food 68,69 Alcoholic liquors and wine / Malt liquors and malt

240 Other personal services, n.e.c.

206 Ownership of dwellings

191 Tour and travel agencies

\section{Total Gifts and contributions expenditure \\ 168 Gifts outside family \\ no match \\ 169 Conributions to church \\ no match \\ Contributions to other \\ 170 institutions \\ no match \\ Other gifts and \\ 171 contributions no match}

\section{Total Other expenditures}

Life insurance and

172 retirement premiums Interest in payment on

173 loans for $\mathrm{HH}$ Other (losses due to fire,

174 theft,legal fees,etc.)

\section{Total Other Disbursements}

Purchase/amortization of

175 real property

176 Cash loan payments Loans granted to person

177 outside the family Amount deposited in

178 banks/investments 179 Other disbursement
Life insurance / Non-life and other insurance 203, 204 activities

222 Other business services no match

Real estate activities with own or leased property
205 and contract basis
no match
no match
200 Banking
no match

Note: The number under the matched column refers to the sectors in the Philippine Input-Output (IO) Table. Some household consumption items have no direct match from the IO sectors and some sectors in the IO table have also no counterpart in the list of household expenditures. Sectors that have no counterpart were left unmatched. For consumption items having more than one IO sector counterparts, we took the average of the carbon intensity and multiply it to the corresponding consumption item to derive the embodied carbon emission. 
Appendix Table 4. Summary statistics of household asset.

\begin{tabular}{|c|c|c|c|c|c|c|c|c|c|c|}
\hline \multirow[b]{3}{*}{ Household Asset } & \multicolumn{5}{|c|}{2000} & \multicolumn{5}{|c|}{2006} \\
\hline & \multicolumn{2}{|c|}{ All Households } & \multicolumn{3}{|c|}{ Means } & \multicolumn{2}{|c|}{ All Households } & \multicolumn{3}{|c|}{ Means } \\
\hline & Score & Mean & $\begin{array}{c}\text { Poorest } \\
40 \% \\
\end{array}$ & $\begin{array}{c}\text { Middle } \\
40 \%\end{array}$ & $\begin{array}{c}\text { Richest } \\
20 \% \\
\end{array}$ & Score & Mean & $\begin{array}{c}\text { Poorest } \\
40 \% \\
\end{array}$ & $\begin{array}{c}\text { Middle } \\
40 \%\end{array}$ & $\begin{array}{c}\text { Richest } \\
20 \%\end{array}$ \\
\hline ownership of radio & 0,10 & 0,73 & 0,61 & 0,79 & 0,84 & 0,09 & 0,60 & 0,47 & 0,63 & 0,74 \\
\hline ownership of television & 0,28 & 0,61 & 0,20 & 0,88 & 1,00 & 0,27 & 0,66 & 0,22 & 0,89 & 1,00 \\
\hline ownership of stereo & 0,22 & 0,25 & 0,04 & 0,26 & 0,72 & 0,21 & 0,24 & 0,03 & 0,23 & 0,62 \\
\hline ownership of vtr/cd/dvd player & 0,26 & 0,25 & 0,01 & 0,24 & 0,85 & 0,27 & 0,43 & 0,05 & 0,50 & 0,92 \\
\hline ownership of refrigerator & 0,29 & 0,39 & 0,02 & 0,52 & 0,98 & 0,29 & 0,37 & 0,02 & 0,38 & 0,96 \\
\hline ownership of washing machine & 0,26 & 0,26 & 0,01 & 0,26 & 0,86 & 0,26 & 0,27 & 0,00 & 0,21 & 0,82 \\
\hline ownership of aircon & 0,15 & 0,05 & 0,00 & 0,03 & 0,23 & 0,17 & 0,06 & 0,00 & 0,01 & 0,26 \\
\hline ownership of sala set & 0,27 & 0,49 & 0,11 & 0,68 & 0,97 & 0,27 & 0,44 & 0,07 & 0,51 & 0,96 \\
\hline ownership of dining set & 0,27 & 0,43 & 0,09 & 0,57 & 0,95 & 0,26 & 0,40 & 0,07 & 0,42 & 0,92 \\
\hline ownership of phone & 0,24 & 0,15 & 0,00 & 0,08 & 0,68 & 0,26 & 0,50 & 0,12 & 0,61 & 0,96 \\
\hline ownership of oven & 0,16 & 0,05 & 0,00 & 0,01 & 0,24 & 0,17 & 0,06 & 0,00 & 0,01 & 0,24 \\
\hline ownership of computer & 0,13 & 0,03 & 0,00 & 0,00 & 0,15 & 0,17 & 0,06 & 0,00 & 0,01 & 0,25 \\
\hline ownership of motorbike & 0,10 & 0,05 & 0,01 & 0,05 & 0,16 & 0,12 & 0,12 & 0,02 & 0,12 & 0,28 \\
\hline ownership of car & 0,17 & 0,06 & 0,00 & 0,01 & 0,29 & 0,17 & 0,06 & 0,00 & 0,01 & 0,26 \\
\hline house made of strong materials & 0,20 & 0,68 & 0,42 & 0,83 & 0,98 & 0,20 & 0,72 & 0,44 & 0,83 & 0,98 \\
\hline house made of light materials & $-0,23$ & 0,46 & 0,78 & 0,30 & 0,05 & $-0,24$ & 0,44 & 0,80 & 0,32 & 0,05 \\
\hline have a flush toilet & 0,24 & 0,72 & 0,40 & 0,94 & 0,99 & 0,23 & 0,74 & 0,41 & 0,91 & 0,99 \\
\hline have a pit/latine toilet & $-0,17$ & 0,18 & 0,37 & 0,05 & 0,00 & $-0,15$ & 0,15 & 0,33 & 0,07 & 0,01 \\
\hline have no toilet & $-0,14$ & 0,10 & 0,23 & 0,02 & 0,00 & $-0,15$ & 0,11 & 0,26 & 0,02 & 0,00 \\
\hline water from water system & 0,19 & 0,45 & 0,22 & 0,53 & 0,82 & 0,19 & 0,44 & 0,18 & 0,48 & 0,78 \\
\hline water from well/pump & $-0,13$ & 0,45 & 0,60 & 0,41 & 0,16 & $-0,13$ & 0,46 & 0,63 & 0,45 & 0,20 \\
\hline water from river, rain, etc. & $-0,09$ & 0,11 & 0,18 & 0,06 & 0,02 & $-0,10$ & 0,10 & 0,19 & 0,07 & 0,02 \\
\hline owning house and lot & 0,07 & 0,69 & 0,62 & 0,71 & 0,82 & 0,06 & 0,72 & 0,65 & 0,73 & 0,82 \\
\hline renting house and lot & 0,06 & 0,06 & 0,02 & 0,08 & 0,10 & 0,05 & 0,06 & 0,02 & 0,08 & 0,10 \\
\hline not owning/renting house $\&$ lot & $-0,10$ & 0,25 & 0,36 & 0,21 & 0,08 & $-0,10$ & 0,22 & 0,34 & 0,20 & 0,08 \\
\hline access to electricity & 0,24 & 0,77 & 0,46 & 0,98 & 1,00 & 0,19 & 0,80 & 0,55 & 0,94 & 0,97 \\
\hline
\end{tabular}

Notes: Each asset takes the value of 1 if households have it or 0 otherwise. Scoring factors is the "weight" assigned to each asset (normalized by its mean and standard deviation) in the linear combinations of the variables that constitute the first principal component.

Source: Authors computation using principal component analysis following the methods of Filmer and Pritchett (2001) 
Appendix Table 5. Household share, mean carbon emission and emission share

\begin{tabular}{|c|c|c|c|c|c|c|}
\hline \multirow[t]{2}{*}{ HH Characteristics } & \multicolumn{4}{|c|}{$\begin{array}{c}\text { Mean } \mathrm{CO}_{2} \text { emission } \\
\text { (in tons) }\end{array}$} & \multicolumn{2}{|c|}{$\mathrm{CO}_{2}$ emission share } \\
\hline & 2000 & 2006 & 2000 & 2006 & 2000 & 2006 \\
\hline \multicolumn{7}{|l|}{ Location } \\
\hline Rural & $40.9 \%$ & $55.1 \%$ & 0.77 & 1.06 & $21.5 \%$ & $31.6 \%$ \\
\hline Urban & $59.1 \%$ & $44.9 \%$ & 1.94 & 2.83 & $78.5 \%$ & $68.4 \%$ \\
\hline \multicolumn{7}{|l|}{ Age of HH head } \\
\hline less than 30 & $5.6 \%$ & $7.1 \%$ & 1.11 & 1.40 & $4.2 \%$ & $5.3 \%$ \\
\hline 30 to 45 & $40.0 \%$ & $39.1 \%$ & 1.40 & 1.70 & $38.3 \%$ & $35.7 \%$ \\
\hline 45 to 60 & $33.1 \%$ & $33.9 \%$ & 1.66 & 2.15 & $37.5 \%$ & $39.1 \%$ \\
\hline above 60 & $21.3 \%$ & $20.0 \%$ & 1.37 & 1.84 & $19.9 \%$ & $19.8 \%$ \\
\hline \multicolumn{7}{|l|}{ Household Size } \\
\hline 1 to 3 members & $22.1 \%$ & $26.3 \%$ & 1.08 & 1.43 & $16.3 \%$ & $20.3 \%$ \\
\hline 4 to 5 members & $35.9 \%$ & $37.8 \%$ & 1.53 & 1.92 & $37.4 \%$ & $39.2 \%$ \\
\hline 6 to 8 members & $34.1 \%$ & $29.6 \%$ & 1.55 & 2.05 & $36.1 \%$ & $32.7 \%$ \\
\hline more than 8 & $7.9 \%$ & $6.2 \%$ & 1.88 & 2.33 & $10.2 \%$ & $7.8 \%$ \\
\hline \multicolumn{7}{|l|}{ Education HH Head } \\
\hline No formal education & $4.6 \%$ & $3.3 \%$ & 0.52 & 0.59 & $1.6 \%$ & $1.0 \%$ \\
\hline Elementary & $40.8 \%$ & $42.1 \%$ & 0.88 & 1.06 & $24.5 \%$ & $24.0 \%$ \\
\hline High School & $32.0 \%$ & $33.1 \%$ & 1.43 & 1.84 & $31.3 \%$ & $32.8 \%$ \\
\hline College & $22.7 \%$ & $21.5 \%$ & 2.75 & 3.64 & $42.6 \%$ & $42.1 \%$ \\
\hline \multicolumn{7}{|l|}{ Income Quintiles } \\
\hline poorest $20 \%$ & & & 0.33 & 0.35 & $5.3 \%$ & $3.2 \%$ \\
\hline 2nd & & & 0.66 & 0.67 & $9.0 \%$ & $7.3 \%$ \\
\hline middle & & & 1.13 & 1.13 & $14.9 \%$ & $12.6 \%$ \\
\hline 4th & & & 1.86 & 2.00 & $24.6 \%$ & $22.2 \%$ \\
\hline richest $20 \%$ & & & 3.76 & 4.63 & $46.3 \%$ & $54.7 \%$ \\
\hline All & & & 1.46 & 1.86 & & \\
\hline
\end{tabular}


Appendix Table 6. Incidence rate ratio for negative binomial regression for baseline analysis.

\begin{tabular}{|c|c|c|c|c|c|c|}
\hline Variables & IRR & IRR & IRR & IRR & IRR & IRR \\
\hline $\log$ GDP pc & $\begin{array}{c}1.251 * * * \\
(0.0803)\end{array}$ & $\begin{array}{c}1.113^{*} \\
(0.0663)\end{array}$ & $\begin{array}{c}1.010 \\
(0.0699)\end{array}$ & $\begin{array}{c}0.954 \\
(0.0648)\end{array}$ & $\begin{array}{c}0.129 * * * \\
(0.0602)\end{array}$ & $\begin{array}{c}0.150^{* * * *} \\
(0.0686)\end{array}$ \\
\hline $\log$ GDP pc_sq & & & & & $\begin{array}{c}1.145^{* * * *} \\
(0.0350)\end{array}$ & $\begin{array}{c}1.130 * * * \\
(0.0335)\end{array}$ \\
\hline energy import & $\begin{array}{l}1.001 * * \\
(0.0004)\end{array}$ & $\begin{array}{c}1.002 * * * \\
(0.0005)\end{array}$ & $\begin{array}{c}1.002 * * * \\
(0.0006)\end{array}$ & $\begin{array}{c}1.002 * * * \\
(0.0006)\end{array}$ & $\begin{array}{l}1.001^{* *} \\
(0.0006)\end{array}$ & $\begin{array}{c}1.002 * * * \\
(0.0006)\end{array}$ \\
\hline population growth & $\begin{array}{c}1.011 \\
(0.0506)\end{array}$ & $\begin{array}{c}1.069 \\
(0.0475)\end{array}$ & $\begin{array}{c}1.144 * * * \\
(0.0546)\end{array}$ & $\begin{array}{c}1.183 * * * \\
(0.0511)\end{array}$ & $\begin{array}{l}1.130^{* *} \\
(0.0561)\end{array}$ & $\begin{array}{c}1.168 * * * * \\
(0.0526)\end{array}$ \\
\hline oil price & $\begin{array}{c}1.023 * * * \\
(0.0037)\end{array}$ & $\begin{array}{c}1.018 * * * \\
(0.0035)\end{array}$ & $\begin{array}{c}1.025 * * * \\
(0.0036)\end{array}$ & $\begin{array}{c}1.022 * * * \\
(0.0035)\end{array}$ & $\begin{array}{c}1.023 * * * \\
(0.0037)\end{array}$ & $\begin{array}{c}1.020^{* * * *} \\
(0.0036)\end{array}$ \\
\hline $\log$ finance dev & $\begin{array}{c}1.180 * * * \\
(0.0515)\end{array}$ & $\begin{array}{c}1.205 * * * \\
(0.0500)\end{array}$ & $\begin{array}{l}1.125 * * \\
(0.0607)\end{array}$ & $\begin{array}{c}1.151 * * * \\
(0.0609)\end{array}$ & $\begin{array}{c}1.153 * * * \\
(0.0601)\end{array}$ & $\begin{array}{c}1.177 * * * * \\
(0.0603)\end{array}$ \\
\hline policy & & $\begin{array}{c}1.746 * * * \\
(0.1218)\end{array}$ & & $\begin{array}{c}1.401 * * * \\
(0.0999)\end{array}$ & & $\begin{array}{c}1.369^{* * * *} \\
(0.0958)\end{array}$ \\
\hline log patents & & & $\begin{array}{c}1.086 * * * \\
(0.0138)\end{array}$ & $\begin{array}{l}1.067 * * * \\
(0.0134)\end{array}$ & $\begin{array}{c}1.078 * * * \\
(0.0139)\end{array}$ & $\begin{array}{l}1.061 * * * \\
(0.0135)\end{array}$ \\
\hline sec enrollment & $\begin{array}{c}1.004 \\
(0.0028)\end{array}$ & $\begin{array}{c}1.003 \\
(0.0027)\end{array}$ & $\begin{array}{c}1.014 * * * \\
(0.0027)\end{array}$ & $\begin{array}{c}1.014 * * * \\
(0.0026)\end{array}$ & $\begin{array}{c}1.014 * * * \\
(0.0027)\end{array}$ & $\begin{array}{c}1.014 * * * \\
(0.0026)\end{array}$ \\
\hline hydro energy & $\begin{array}{c}0.657 * * * \\
(0.0548)\end{array}$ & $\begin{array}{c}0.731 * * * \\
(0.0574)\end{array}$ & $\begin{array}{c}0.448 * * * \\
(0.0310)\end{array}$ & $\begin{array}{c}0.473 * * * \\
(0.0321)\end{array}$ & $\begin{array}{c}0.430 * * * \\
(0.0308)\end{array}$ & $\begin{array}{c}0.454 * * * \\
(0.0320)\end{array}$ \\
\hline oil prod'n & $\begin{array}{c}0.986^{* * * *} \\
(0.0051)\end{array}$ & $\begin{array}{c}0.992^{*} \\
(0.0050)\end{array}$ & $\begin{array}{c}0.977 * * \\
(0.0095)\end{array}$ & $\begin{array}{c}0.981 * * \\
(0.0090)\end{array}$ & $\begin{array}{c}0.971 * * * \\
(0.0100)\end{array}$ & $\begin{array}{c}0.975^{* *} \\
(0.0096)\end{array}$ \\
\hline coal prod'n & $\begin{array}{c}1.094 * * * \\
(0.0225)\end{array}$ & $\begin{array}{c}1.121 * * * \\
(0.0257)\end{array}$ & $\begin{array}{l}1.083^{* *} \\
(0.0383)\end{array}$ & $\begin{array}{c}1.110 * * * \\
(0.0406)\end{array}$ & $\begin{array}{c}1.103 * * * \\
(0.0403)\end{array}$ & $\begin{array}{c}1.126^{* * * *} \\
(0.0423)\end{array}$ \\
\hline FDI & & & $\begin{array}{c}0.955^{* *} \\
(0.0213)\end{array}$ & $\begin{array}{c}0.955^{* *} \\
(0.0202)\end{array}$ & $\begin{array}{l}0.951 * * \\
(0.0205)\end{array}$ & $\begin{array}{c}0.951 * * \\
(0.0196)\end{array}$ \\
\hline ODA & & & $\begin{array}{c}0.988 \\
(0.0106)\end{array}$ & $\begin{array}{c}0.985 \\
(0.0105)\end{array}$ & $\begin{array}{c}0.977 * * \\
(0.0113)\end{array}$ & $\begin{array}{c}0.976^{* *} \\
(0.0111)\end{array}$ \\
\hline coast & $\begin{array}{c}2.487 * * * \\
(0.3371)\end{array}$ & $\begin{array}{c}2.418 * * * \\
(0.3278)\end{array}$ & $\begin{array}{c}2.592 * * * \\
(0.3858)\end{array}$ & $\begin{array}{c}2.554 * * * \\
(0.3791)\end{array}$ & $\begin{array}{c}2.549 * * * \\
(0.3819)\end{array}$ & $\begin{array}{c}2.512 * * * \\
(0.3737)\end{array}$ \\
\hline $\begin{array}{l}\text { regional dummies } \\
\text { year dummies }\end{array}$ & $\begin{array}{l}\text { yes } \\
\text { yes }\end{array}$ & $\begin{array}{l}\text { yes } \\
\text { yes }\end{array}$ & $\begin{array}{l}\text { yes } \\
\text { yes }\end{array}$ & $\begin{array}{l}\text { yes } \\
\text { yes }\end{array}$ & $\begin{array}{l}\text { yes } \\
\text { yes }\end{array}$ & $\begin{array}{l}\text { yes } \\
\text { yes }\end{array}$ \\
\hline constant & $\begin{array}{c}0.003 * * * \\
(0.0017)\end{array}$ & $\begin{array}{c}0.008 * * * \\
(0.0043)\end{array}$ & $\begin{array}{c}0.007 * * * \\
(0.0038)\end{array}$ & $\begin{array}{c}0.011 * * * \\
(0.0061)\end{array}$ & $\begin{array}{c}17.965 \\
(33.6554)\end{array}$ & $\begin{array}{c}12.970 \\
(23.8730)\end{array}$ \\
\hline observations & 1,386 & 1,386 & 1,232 & 1,232 & 1,232 & 1,232 \\
\hline
\end{tabular}

Robust standard errors in parentheses

$* * * \mathrm{p}<0.01, * * \mathrm{p}<0.05, * \mathrm{p}<0.1$ 
Appendix Table 7. Incidence rate ratio capturing the effect of Kyoto protocol on diversification

\begin{tabular}{|c|c|c|c|c|c|c|}
\hline Variables & IRR & IRR & IRR & IRR & IRR & IRR \\
\hline $\log$ GDP pc & $\begin{array}{c}1.258 * * * \\
(0.0840)\end{array}$ & $\begin{array}{c}1.109 * \\
(0.0682)\end{array}$ & $\begin{array}{c}1.025 \\
(0.0742)\end{array}$ & $\begin{array}{c}0.963 \\
(0.0684)\end{array}$ & $\begin{array}{c}0.143 * * * \\
(0.0696)\end{array}$ & $\begin{array}{c}0.166 * * * \\
(0.0797)\end{array}$ \\
\hline $\log$ GDP pc_sq & & & & & $\begin{array}{c}1.139 * * * \\
(0.0360)\end{array}$ & $\begin{array}{c}1.123 * * * \\
(0.0347)\end{array}$ \\
\hline energy import & $\begin{array}{l}1.001 * * \\
(0.0004)\end{array}$ & $\begin{array}{c}1.002 * * * \\
(0.0005)\end{array}$ & $\begin{array}{c}1.002 * * * \\
(0.0006)\end{array}$ & $\begin{array}{c}1.002 * * * \\
(0.0006)\end{array}$ & $\begin{array}{l}1.001 * * \\
(0.0006)\end{array}$ & $\begin{array}{c}1.002 * * * \\
(0.0006)\end{array}$ \\
\hline population growth & $\begin{array}{c}0.986 \\
(0.0513)\end{array}$ & $\begin{array}{c}1.046 \\
(0.0492)\end{array}$ & $\begin{array}{c}1.100 * \\
(0.0585)\end{array}$ & $\begin{array}{c}1.143^{* * *} * \\
(0.0554)\end{array}$ & $\begin{array}{c}1.086 \\
(0.0598)\end{array}$ & $\begin{array}{l}1.127 * * \\
(0.0574)\end{array}$ \\
\hline oil price & $\begin{array}{l}1.003 * * \\
(0.0011)\end{array}$ & $\begin{array}{c}1.000 \\
(0.0011)\end{array}$ & $\begin{array}{c}1.003 * * * \\
(0.0011)\end{array}$ & $\begin{array}{c}1.002 \\
(0.0011)\end{array}$ & $\begin{array}{c}1.003 * * * \\
(0.0011)\end{array}$ & $\begin{array}{c}1.001 \\
(0.0011)\end{array}$ \\
\hline log finance dev & $\begin{array}{c}1.155^{* * *} * \\
(0.0503)\end{array}$ & $\begin{array}{c}1.183 * * * \\
(0.0489)\end{array}$ & $\begin{array}{c}1.100 * \\
(0.0579)\end{array}$ & $\begin{array}{l}1.131 * * \\
(0.0583)\end{array}$ & $\begin{array}{l}1.127 * * \\
(0.0576)\end{array}$ & $\begin{array}{c}1.156 * * * \\
(0.0582)\end{array}$ \\
\hline policy & & $\begin{array}{c}1.803 * * * \\
(0.1306)\end{array}$ & & $\begin{array}{c}1.440 * * * \\
(0.1086)\end{array}$ & & $\begin{array}{c}1.411 * * * \\
(0.1053)\end{array}$ \\
\hline log patents & & & $\begin{array}{c}1.091 * * * \\
(0.0140)\end{array}$ & $\begin{array}{c}1.071 * * * \\
(0.0135)\end{array}$ & $\begin{array}{c}1.084 * * * \\
(0.0140)\end{array}$ & $\begin{array}{c}1.066 * * * \\
(0.0136)\end{array}$ \\
\hline sec enrollment & $\begin{array}{l}1.007 * * \\
(0.0027)\end{array}$ & $\begin{array}{l}1.006 * * \\
(0.0027)\end{array}$ & $\begin{array}{c}1.016 * * * \\
(0.0026)\end{array}$ & $\begin{array}{c}1.016 * * * \\
(0.0025)\end{array}$ & $\begin{array}{c}1.016 * * * \\
(0.0027)\end{array}$ & $\begin{array}{c}1.016 * * * \\
(0.0026)\end{array}$ \\
\hline Kyoto protocol & $\begin{array}{c}1.839 * * * \\
(0.1497)\end{array}$ & $\begin{array}{c}1.687 * * * \\
(0.1380)\end{array}$ & $\begin{array}{c}1.835 * * * \\
(0.1562)\end{array}$ & $\begin{array}{c}1.732 * * * \\
(0.1504)\end{array}$ & $\begin{array}{c}1.819 * * * \\
(0.1528)\end{array}$ & $\begin{array}{c}1.723 * * * \\
(0.1480)\end{array}$ \\
\hline hydro energy & $\begin{array}{c}0.655 * * * \\
(0.0556)\end{array}$ & $\begin{array}{c}0.735 * * * \\
(0.0590)\end{array}$ & $\begin{array}{c}0.436 * * * \\
(0.0320)\end{array}$ & $\begin{array}{c}0.465 * * * \\
(0.0331)\end{array}$ & $\begin{array}{c}0.419 * * * \\
(0.0317)\end{array}$ & $\begin{array}{c}0.448 * * * \\
(0.0329)\end{array}$ \\
\hline oil prod'n & $\begin{array}{c}0.986 * * * \\
(0.0050)\end{array}$ & $\begin{array}{c}0.992 \\
(0.0049)\end{array}$ & $\begin{array}{c}0.977 * * \\
(0.0091)\end{array}$ & $\begin{array}{c}0.980 * * \\
(0.0086)\end{array}$ & $\begin{array}{c}0.971 * * * \\
(0.0096)\end{array}$ & $\begin{array}{r}0.975 * * * \\
(0.0092)\end{array}$ \\
\hline coal prod'n & $\begin{array}{c}1.089 * * * \\
(0.0218)\end{array}$ & $\begin{array}{c}1.118 * * * \\
(0.0251)\end{array}$ & $\begin{array}{c}1.067 * \\
(0.0360)\end{array}$ & $\begin{array}{c}1.095 * * * \\
(0.0379)\end{array}$ & $\begin{array}{l}1.083 * * \\
(0.0375)\end{array}$ & $\begin{array}{c}1.108 * * * \\
(0.0393)\end{array}$ \\
\hline FDI & & & $\begin{array}{c}0.967 * \\
(0.0182)\end{array}$ & $\begin{array}{c}0.967 * \\
(0.0169)\end{array}$ & $\begin{array}{c}0.963 * * \\
(0.0175)\end{array}$ & $\begin{array}{l}0.963 * * \\
(0.0164)\end{array}$ \\
\hline ODA & & & $\begin{array}{c}0.990 \\
(0.0115)\end{array}$ & $\begin{array}{c}0.987 \\
(0.0113)\end{array}$ & $\begin{array}{c}0.980 \\
(0.0122)\end{array}$ & $\begin{array}{c}0.979 * \\
(0.0118)\end{array}$ \\
\hline coast & $\begin{array}{c}2.524 * * * \\
(0.3366)\end{array}$ & $\begin{array}{c}2.458 * * * \\
(0.3299)\end{array}$ & $\begin{array}{c}2.661 * * * \\
(0.3906)\end{array}$ & $\begin{array}{c}2.609 * * * \\
(0.3827)\end{array}$ & $\begin{array}{c}2.613 * * * \\
(0.3837)\end{array}$ & $\begin{array}{c}2.564 * * * \\
(0.3752)\end{array}$ \\
\hline $\begin{array}{l}\text { regional dummies } \\
\text { year dummies }\end{array}$ & $\begin{array}{l}\text { yes } \\
\text { yes }\end{array}$ & $\begin{array}{l}\text { yes } \\
\text { yes }\end{array}$ & $\begin{array}{l}\text { yes } \\
\text { yes }\end{array}$ & $\begin{array}{l}\text { yes } \\
\text { yes }\end{array}$ & $\begin{array}{l}\text { yes } \\
\text { yes }\end{array}$ & $\begin{array}{l}\text { yes } \\
\text { yes }\end{array}$ \\
\hline constant & $\begin{array}{c}0.009 * * * \\
(0.0041)\end{array}$ & $\begin{array}{c}0.023 * * * \\
(0.0097)\end{array}$ & $\begin{array}{c}0.022 * * * \\
(0.0109)\end{array}$ & $\begin{array}{c}0.034 * * * \\
(0.0168)\end{array}$ & $\begin{array}{l}38.795 * \\
(75.1252)\end{array}$ & $\begin{array}{l}26.373 * \\
(50.2772)\end{array}$ \\
\hline observations & 1,386 & 1,386 & 1,232 & 1,232 & 1,232 & 1,232 \\
\hline
\end{tabular}


Appendix Table 8. Estimation using Two-Parts Model with diversity index as dependent variable.

\begin{tabular}{|c|c|c|c|c|c|c|c|c|}
\hline VARIABLES & $\operatorname{logit}$ & ols & logit & ols & $\operatorname{logit}$ & ols & logit & ols \\
\hline $\log$ GDP pc & $\begin{array}{c}-0.107 \\
(0.1307)\end{array}$ & $\begin{array}{l}0.191^{* * *} \\
(0.0287)\end{array}$ & $\begin{array}{c}-0.180 \\
(0.1341)\end{array}$ & $\begin{array}{l}0.173^{* * *} \\
(0.0285)\end{array}$ & $\begin{array}{l}-2.936^{* *} \\
(1.2231)\end{array}$ & $\begin{array}{c}-0.843^{* * *} \\
(0.2644)\end{array}$ & $\begin{array}{l}-2.931^{* *} \\
(1.2232)\end{array}$ & $\begin{array}{c}-0.793^{* * *} \\
(0.2683)\end{array}$ \\
\hline $\log$ GDP pc_sq & & & & & $\begin{array}{l}0.178^{* *} \\
(0.0874)\end{array}$ & $\begin{array}{l}0.068^{* * * *} \\
(0.0180)\end{array}$ & $\begin{array}{l}0.176^{* *} \\
(0.0877)\end{array}$ & $\begin{array}{l}0.064^{* * * *} \\
(0.0183)\end{array}$ \\
\hline energy import & $\begin{array}{c}0.001 \\
(0.0013)\end{array}$ & $\begin{array}{c}-0.001^{* * *} \\
(0.0002)\end{array}$ & $\begin{array}{c}0.001 \\
(0.0014)\end{array}$ & $\begin{array}{c}-0.000^{\text {*** }} \\
(0.0002)\end{array}$ & $\begin{array}{c}0.002 \\
(0.0022)\end{array}$ & $\begin{array}{l}-0.001^{* * *} \\
(0.0002)\end{array}$ & $\begin{array}{c}0.002 \\
(0.0022)\end{array}$ & $\begin{array}{l}-0.001^{\text {**** }} \\
(0.0002)\end{array}$ \\
\hline population growth & $\begin{array}{l}0.225^{* *} \\
(0.1110)\end{array}$ & $\begin{array}{l}0.100^{* * *} \\
(0.0330)\end{array}$ & $\begin{array}{l}0.240^{* *} \\
(0.1084)\end{array}$ & $\begin{array}{l}0.098^{* * *} \\
(0.0328)\end{array}$ & $\begin{array}{c}0.200 \\
(0.1361)\end{array}$ & $\begin{array}{l}0.086^{* * *} \\
(0.0291)\end{array}$ & $\begin{array}{c}0.207 \\
(0.1360)\end{array}$ & $\begin{array}{l}0.087^{* * *} \\
(0.0299)\end{array}$ \\
\hline oil price & $\begin{array}{l}0.048^{* * *} \\
(0.0137)\end{array}$ & $\begin{array}{l}0.005^{* * *} \\
(0.0014)\end{array}$ & $\begin{array}{l}0.041^{* * *} \\
(0.0139)\end{array}$ & $\begin{array}{l}0.004^{* *} \\
(0.0015)\end{array}$ & $\begin{array}{l}0.047^{* * *} \\
(0.0180)\end{array}$ & $\begin{array}{l}0.003^{* *} \\
(0.0016)\end{array}$ & $\begin{array}{l}0.045^{* *} \\
(0.0176)\end{array}$ & $\begin{array}{c}0.003^{*} \\
(0.0016)\end{array}$ \\
\hline policy & & & $\begin{array}{l}0.602^{* * *} \\
(0.2304)\end{array}$ & $\begin{array}{l}0.095^{* *} \\
(0.0370)\end{array}$ & & & $\begin{array}{c}0.197 \\
(0.2713)\end{array}$ & $\begin{array}{c}0.046 \\
(0.0404)\end{array}$ \\
\hline log patents & $\begin{array}{l}0.207^{* * *} \\
(0.0303)\end{array}$ & $\begin{array}{l}0.012^{* * *} \\
(0.0040)\end{array}$ & $\begin{array}{l}0.193^{* * * *} \\
(0.0311)\end{array}$ & $\begin{array}{c}0.008^{*} \\
(0.0043)\end{array}$ & $\begin{array}{l}0.126^{* * *} \\
(0.0368)\end{array}$ & $\begin{array}{c}0.009^{*} \\
(0.0047)\end{array}$ & $\begin{array}{l}0.122^{* * *} \\
(0.0374)\end{array}$ & $\begin{array}{c}0.007 \\
(0.0049)\end{array}$ \\
\hline log finance dev & $\begin{array}{l}0.454^{* * * *} \\
(0.1085)\end{array}$ & $\begin{array}{c}0.019 \\
(0.0218)\end{array}$ & $\begin{array}{l}0.481^{* * * *} \\
(0.1113)\end{array}$ & $\begin{array}{c}0.019 \\
(0.0216)\end{array}$ & $\begin{array}{l}0.559^{\text {**** }} \\
(0.1213)\end{array}$ & $\begin{array}{c}-0.033 \\
(0.0204)\end{array}$ & $\begin{array}{l}0.569^{* * *} \\
(0.1224)\end{array}$ & $\begin{array}{c}-0.033 \\
(0.0204)\end{array}$ \\
\hline sec enrollment & $\begin{array}{c}0.005 \\
(0.0064)\end{array}$ & $\begin{array}{l}-0.002^{*} \\
(0.0010)\end{array}$ & $\begin{array}{c}0.005 \\
(0.0064)\end{array}$ & $\begin{array}{l}-0.002 \\
(0.0010)\end{array}$ & $\begin{array}{l}0.036^{\text {*** }} \\
(0.0075)\end{array}$ & $\begin{array}{c}0.000 \\
(0.0012)\end{array}$ & $\begin{array}{l}0.036^{* * *} \\
(0.0075)\end{array}$ & $\begin{array}{l}-0.000 \\
(0.0012)\end{array}$ \\
\hline hydro energy & $\begin{array}{c}-0.509^{* * *} \\
(0.1232)\end{array}$ & $\begin{array}{l}-0.016 \\
(0.0114)\end{array}$ & $\begin{array}{c}-0.466^{\text {*** }} \\
(0.1199)\end{array}$ & $\begin{array}{l}-0.011 \\
(0.0112)\end{array}$ & $\begin{array}{c}-1.867^{* * *} \\
(0.1780)\end{array}$ & $\begin{array}{c}-0.115^{* * *} \\
(0.0333)\end{array}$ & $\begin{array}{c}-1.829^{* * *} \\
(0.1891)\end{array}$ & $\begin{array}{l}-0.100^{\text {*** }} \\
(0.0362)\end{array}$ \\
\hline oil prod'n & $\begin{array}{c}-0.048^{* * * *} \\
(0.0163)\end{array}$ & $\begin{array}{c}-0.011^{* * * *} \\
(0.0023)\end{array}$ & $\begin{array}{c}-0.045^{\text {*** }} \\
(0.0157)\end{array}$ & $\begin{array}{l}-0.010^{* * * *} \\
(0.0022)\end{array}$ & $\begin{array}{l}-0.038^{*} \\
(0.0221)\end{array}$ & $\begin{array}{c}-0.012^{* * * *} \\
(0.0028)\end{array}$ & $\begin{array}{l}-0.036^{*} \\
(0.0214)\end{array}$ & $\begin{array}{c}-0.012^{\text {**** }} \\
(0.0028)\end{array}$ \\
\hline coal prod'n & $\begin{array}{c}0.058 \\
(0.0625)\end{array}$ & $\begin{array}{l}-0.029^{* * *} \\
(0.0099)\end{array}$ & $\begin{array}{c}0.075 \\
(0.0646)\end{array}$ & $\begin{array}{l}-0.023^{* *} \\
(0.0094)\end{array}$ & $\begin{array}{c}0.133 \\
(0.1028)\end{array}$ & $\begin{array}{l}-0.006 \\
(0.0127)\end{array}$ & $\begin{array}{c}0.132 \\
(0.1003)\end{array}$ & $\begin{array}{c}-0.004 \\
(0.0124)\end{array}$ \\
\hline FDI & & & & & $\begin{array}{l}-0.121^{* *} \\
(0.0511)\end{array}$ & $\begin{array}{l}-0.000 \\
(0.0037)\end{array}$ & $\begin{array}{l}-0.118^{* *} \\
(0.0513)\end{array}$ & $\begin{array}{l}-0.001 \\
(0.0038)\end{array}$ \\
\hline ODA & & & & & $\begin{array}{l}-0.013 \\
(0.0166)\end{array}$ & $\begin{array}{l}-0.006 \\
(0.0036)\end{array}$ & $\begin{array}{l}-0.014 \\
(0.0165)\end{array}$ & $\begin{array}{l}-0.006^{*} \\
(0.0037)\end{array}$ \\
\hline coast & $\begin{array}{l}1.090^{* * *} \\
(0.2534)\end{array}$ & $\begin{array}{c}0.039 \\
(0.0463)\end{array}$ & $\begin{array}{l}1.115^{* * *} \\
(0.2528)\end{array}$ & $\begin{array}{c}0.042 \\
(0.0472)\end{array}$ & $\begin{array}{l}2.005^{\text {**** }} \\
(0.3294)\end{array}$ & $\begin{array}{c}0.072 \\
(0.0704)\end{array}$ & $\begin{array}{l}1.992^{* * *} \\
(0.3287)\end{array}$ & $\begin{array}{c}0.063 \\
(0.0711)\end{array}$ \\
\hline $\begin{array}{l}\text { regional dummies } \\
\text { year dummies }\end{array}$ & $\begin{array}{l}\text { yes } \\
\text { yes }\end{array}$ & $\begin{array}{l}\text { yes } \\
\text { yes }\end{array}$ & $\begin{array}{l}\text { yes } \\
\text { yes }\end{array}$ & $\begin{array}{l}\text { yes } \\
\text { yes }\end{array}$ & $\begin{array}{l}\text { yes } \\
\text { yes }\end{array}$ & $\begin{array}{l}\text { yes } \\
\text { yes }\end{array}$ & $\begin{array}{l}\text { yes } \\
\text { yes }\end{array}$ & $\begin{array}{l}\text { yes } \\
\text { yes }\end{array}$ \\
\hline constant & $\begin{array}{l}-6.812^{* * * *} \\
(1.1709)\end{array}$ & $\begin{array}{l}-0.977^{* * *} \\
(0.2524)\end{array}$ & $\begin{array}{l}-6.086^{* * *} \\
(1.2001)\end{array}$ & $\begin{array}{l}-0.800^{* * *} \\
(0.2450)\end{array}$ & $\begin{array}{c}2.093 \\
(4.5694)\end{array}$ & $\begin{array}{l}3.132^{* * *} \\
(1.0189)\end{array}$ & $\begin{array}{c}2.251 \\
(4.5448)\end{array}$ & $\begin{array}{l}3.006^{* * *} \\
(1.0345)\end{array}$ \\
\hline $\begin{array}{l}\text { observations } \\
\text { Pseudo R2 }\end{array}$ & $\begin{array}{l}1,386 \\
0,3738\end{array}$ & 629 & $\begin{array}{r}1,386 \\
0,3772\end{array}$ & 629 & $\begin{array}{c}1,232 \\
0,4510\end{array}$ & 542 & $\begin{array}{c}1,232 \\
0,4513\end{array}$ & 542 \\
\hline $\mathrm{R} 2$ & & 0.3078 & & 0.3180 & & 0.3243 & & 0.3265 \\
\hline
\end{tabular}

Robust standard errors in parentheses

$* * * \mathrm{p}<0.01, * * \mathrm{p}<0.05, * \mathrm{p}<0.1$ 
Appendix Table 9. Estimation using Two-Parts Model controlling for Kyoto Protocol.

\begin{tabular}{|c|c|c|c|c|c|c|c|c|}
\hline VARIABLES & logit & ols & logit & ols & logit & ols & logit & ols \\
\hline $\log$ GDP pc & $\begin{array}{c}-0.126 \\
(0.1296)\end{array}$ & $\begin{array}{l}0.186^{* * *} \\
(0.0289)\end{array}$ & $\begin{array}{c}-0.208 \\
(0.1330)\end{array}$ & $\begin{array}{l}0.167^{* * *} \\
(0.0286)\end{array}$ & $\begin{array}{l}-3.323^{* * *} \\
(1.2599)\end{array}$ & $\begin{array}{l}-0.773^{* * * *} \\
(0.2570)\end{array}$ & $\begin{array}{l}-3.310^{* * * *} \\
(1.2622)\end{array}$ & $\begin{array}{l}-0.723^{* * *} \\
(0.2609)\end{array}$ \\
\hline $\log$ GDP pc_sq & & & & & $\begin{array}{l}0.208^{* *} \\
(0.0890)\end{array}$ & $\begin{array}{l}0.063^{* * *} \\
(0.0175)\end{array}$ & $\begin{array}{l}0.204^{* *} \\
(0.0896)\end{array}$ & $\begin{array}{l}0.059^{* * * *} \\
(0.0178)\end{array}$ \\
\hline energy import & $\begin{array}{c}0.001 \\
(0.0011)\end{array}$ & $\begin{array}{l}-0.001^{* * *} \\
(0.0002)\end{array}$ & $\begin{array}{c}0.001 \\
(0.0013)\end{array}$ & $\begin{array}{l}-0.000^{* * *} \\
(0.0002)\end{array}$ & $\begin{array}{c}0.002 \\
(0.0019)\end{array}$ & $\begin{array}{l}-0.001^{* * * *} \\
(0.0002)\end{array}$ & $\begin{array}{c}0.002 \\
(0.0019)\end{array}$ & $\begin{array}{l}-0.000^{* *} \\
(0.0002)\end{array}$ \\
\hline population growth & $\begin{array}{c}0.188 \\
(0.1166)\end{array}$ & $\begin{array}{l}0.095^{* * *} \\
(0.0312)\end{array}$ & $\begin{array}{l}0.205^{*} \\
(0.1127)\end{array}$ & $\begin{array}{l}0.093^{* * *} \\
(0.0309)\end{array}$ & $\begin{array}{c}0.134 \\
(0.1480)\end{array}$ & $\begin{array}{l}0.076^{* * *} \\
(0.0265)\end{array}$ & $\begin{array}{c}0.146 \\
(0.1460)\end{array}$ & $\begin{array}{l}0.077^{* * *} \\
(0.0273)\end{array}$ \\
\hline oil price & $\begin{array}{l}0.010^{* * *} \\
(0.0038)\end{array}$ & $\begin{array}{c}0.000 \\
(0.0006)\end{array}$ & $\begin{array}{l}0.008^{*} \\
(0.0040)\end{array}$ & $\begin{array}{c}-0.000 \\
(0.0006)\end{array}$ & $\begin{array}{l}0.012^{* * *} \\
(0.0045)\end{array}$ & $\begin{array}{c}-0.001 \\
(0.0006)\end{array}$ & $\begin{array}{l}0.011^{* *} \\
(0.0047)\end{array}$ & $\begin{array}{c}-0.001 \\
(0.0006)\end{array}$ \\
\hline policy & & & $\begin{array}{l}0.673^{* * *} \\
(0.2220)\end{array}$ & $\begin{array}{l}0.097^{* * *} \\
(0.0366)\end{array}$ & & & $\begin{array}{c}0.274 \\
(0.2624)\end{array}$ & $\begin{array}{c}0.048 \\
(0.0405)\end{array}$ \\
\hline $\log$ patents & $\begin{array}{l}0.204^{* * *} \\
(0.0295)\end{array}$ & $\begin{array}{l}0.012^{* * *} \\
(0.0039)\end{array}$ & $\begin{array}{l}0.188^{* * *} \\
(0.0302)\end{array}$ & $\begin{array}{l}0.008^{*} \\
(0.0041)\end{array}$ & $\begin{array}{l}0.129^{* * *} \\
(0.0350)\end{array}$ & $\begin{array}{l}0.010^{* *} \\
(0.0044)\end{array}$ & $\begin{array}{l}0.122^{* * * *} \\
(0.0356)\end{array}$ & $\begin{array}{l}0.008^{*} \\
(0.0047)\end{array}$ \\
\hline $\log$ finance dev & $\begin{array}{l}0.436^{* * *} \\
(0.1021)\end{array}$ & $\begin{array}{c}0.016 \\
(0.0215)\end{array}$ & $\begin{array}{l}0.463^{* * *} \\
(0.1043)\end{array}$ & $\begin{array}{c}0.016 \\
(0.0213)\end{array}$ & $\begin{array}{l}0.543^{* * *} \\
(0.1153)\end{array}$ & $\begin{array}{l}-0.035^{*} \\
(0.0196)\end{array}$ & $\begin{array}{l}0.554^{* * *} \\
(0.1153)\end{array}$ & $\begin{array}{l}-0.034^{*} \\
(0.0196)\end{array}$ \\
\hline sec enrollment & $\begin{array}{l}0.012^{*} \\
(0.0061)\end{array}$ & $\begin{array}{l}-0.001 \\
(0.0009)\end{array}$ & $\begin{array}{l}0.011^{*} \\
(0.0060)\end{array}$ & $\begin{array}{l}-0.001 \\
(0.0009)\end{array}$ & $\begin{array}{l}0.042^{* * *} \\
(0.0074)\end{array}$ & $\begin{array}{c}0.000 \\
(0.0011)\end{array}$ & $\begin{array}{l}0.041^{* * * *} \\
(0.0074)\end{array}$ & $\begin{array}{c}-0.000 \\
(0.0011)\end{array}$ \\
\hline Kyoto protocol & $\begin{array}{l}1.095^{* * *} \\
(0.2077)\end{array}$ & $\begin{array}{l}0.169^{* * * *} \\
(0.0335)\end{array}$ & $\begin{array}{l}1.007^{* * *} \\
(0.2074)\end{array}$ & $\begin{array}{l}0.152^{* * * *} \\
(0.0335)\end{array}$ & $\begin{array}{l}0.901^{* * *} \\
(0.2400)\end{array}$ & $\begin{array}{l}0.159^{* * *} \\
(0.0357)\end{array}$ & $\begin{array}{l}0.852^{* * *} \\
(0.2433)\end{array}$ & $\begin{array}{l}0.153^{\text {*** }} \\
(0.0360)\end{array}$ \\
\hline hydro energy & $\begin{array}{l}-0.458^{* * * *} \\
(0.1283)\end{array}$ & $\begin{array}{l}-0.009 \\
(0.0099)\end{array}$ & $\begin{array}{l}-0.413^{* * *} \\
(0.1257)\end{array}$ & $\begin{array}{l}-0.004 \\
(0.0096)\end{array}$ & $\begin{array}{l}-1.822^{* * *} \\
(0.1781)\end{array}$ & $\begin{array}{l}-0.099^{* * * *} \\
(0.0326)\end{array}$ & $\begin{array}{l}-1.770^{* * * *} \\
(0.1871)\end{array}$ & $\begin{array}{l}-0.084^{* *} \\
(0.0353)\end{array}$ \\
\hline oil prod'n & $\begin{array}{c}-0.046^{* * *} \\
(0.0168)\end{array}$ & $\begin{array}{l}-0.011^{* * *} \\
(0.0023)\end{array}$ & $\begin{array}{l}-0.042^{* * *} \\
(0.0161)\end{array}$ & $\begin{array}{l}-0.010^{* * * *} \\
(0.0022)\end{array}$ & $\begin{array}{l}-0.041^{*} \\
(0.0226)\end{array}$ & $\begin{array}{l}-0.011^{* * *} \\
(0.0028)\end{array}$ & $\begin{array}{l}-0.039^{*} \\
(0.0218)\end{array}$ & $\begin{array}{l}-0.011^{* * *} \\
(0.0027)\end{array}$ \\
\hline coal prod'n & $\begin{array}{c}0.049 \\
(0.0615)\end{array}$ & $\begin{array}{l}-0.031^{* * *} \\
(0.0097)\end{array}$ & $\begin{array}{c}0.067 \\
(0.0632)\end{array}$ & $\begin{array}{l}-0.026^{* * *} \\
(0.0091)\end{array}$ & $\begin{array}{c}0.116 \\
(0.1004)\end{array}$ & $\begin{array}{c}-0.008 \\
(0.0122)\end{array}$ & $\begin{array}{c}0.114 \\
(0.0970)\end{array}$ & $\begin{array}{c}-0.005 \\
(0.0120)\end{array}$ \\
\hline FDI & & & & & $\begin{array}{l}-0.109^{* *} \\
(0.0440)\end{array}$ & $\begin{array}{c}-0.000 \\
(0.0036)\end{array}$ & $\begin{array}{l}-0.105^{* *} \\
(0.0436)\end{array}$ & $\begin{array}{c}-0.001 \\
(0.0036)\end{array}$ \\
\hline ODA & & & & & $\begin{array}{c}-0.006 \\
(0.0171)\end{array}$ & $\begin{array}{c}-0.004 \\
(0.0032)\end{array}$ & $\begin{array}{c}-0.007 \\
(0.0171)\end{array}$ & $\begin{array}{c}-0.004 \\
(0.0033)\end{array}$ \\
\hline coast & $\begin{array}{l}1.124^{* * *} \\
(0.2501)\end{array}$ & $\begin{array}{c}0.038 \\
(0.0445)\end{array}$ & $\begin{array}{l}1.155^{* * *} \\
(0.2503)\end{array}$ & $\begin{array}{c}0.040 \\
(0.0456)\end{array}$ & $\begin{array}{l}2.014^{* * *} \\
(0.3204)\end{array}$ & $\begin{array}{c}0.061 \\
(0.0677)\end{array}$ & $\begin{array}{l}1.999^{* * *} \\
(0.3195)\end{array}$ & $\begin{array}{c}0.050 \\
(0.0683)\end{array}$ \\
\hline regional dummies & yes & yes & yes & yes & yes & yes & yes & yes \\
\hline time dummies & no & no & no & no & no & no & no & no \\
\hline constant & $\begin{array}{l}-4.850^{* * * *} \\
(0.8402)\end{array}$ & $\begin{array}{l}-0.640^{* * *} \\
(0.2251)\end{array}$ & $\begin{array}{l}-4.230^{* * * *} \\
(0.8674)\end{array}$ & $\begin{array}{l}-0.479^{* *} \\
(0.2173)\end{array}$ & $\begin{array}{c}5.228 \\
(4.5914)\end{array}$ & $\begin{array}{l}3.171^{* * *} \\
(0.9788)\end{array}$ & $\begin{array}{c}5.360 \\
(4.5835)\end{array}$ & $\begin{array}{l}3.045^{\text {*** }} \\
(0.9932)\end{array}$ \\
\hline observations & 1,386 & 629 & 1,386 & 629 & 1,232 & 542 & 1,232 & 542 \\
\hline
\end{tabular}


Appendix Table 10. Effect of Kyoto Protocol using time dummies.

\begin{tabular}{|c|c|c|c|c|c|c|c|c|c|}
\hline Variable & Negbin & Negbin & Negbin & Negbin & Variable & Negbin & Negbin & Negbin & Negbin \\
\hline $\operatorname{lgdpc}$ & $\begin{array}{l}0.22^{* * * *} \\
(0.064)\end{array}$ & $\begin{array}{c}0.11^{*} \\
(0.060)\end{array}$ & $\begin{array}{l}-2.05^{* * *} \\
(0.467)\end{array}$ & $\begin{array}{l}-1.90^{* * *} \\
(0.459)\end{array}$ & y86 & $\begin{array}{l}-0.60^{* *} \\
(0.278)\end{array}$ & $\begin{array}{l}-0.57^{* *} \\
(0.270)\end{array}$ & $\begin{array}{l}-0.71^{* *} \\
(0.284)\end{array}$ & $\begin{array}{l}-0.68^{* *} \\
(0.275)\end{array}$ \\
\hline $\operatorname{lgdpc} 2$ & & & $\begin{array}{l}0.14^{* * * *} \\
(0.031)\end{array}$ & $\begin{array}{l}0.12^{* * *} \\
(0.030)\end{array}$ & y87 & $\begin{array}{l}-0.66^{* *} \\
(0.294)\end{array}$ & $\begin{array}{l}-0.61^{* *} \\
(0.289)\end{array}$ & $\begin{array}{l}-0.78^{* * *} \\
(0.299)\end{array}$ & $\begin{array}{l}-0.74^{* *} \\
(0.292)\end{array}$ \\
\hline energy imp & $\begin{array}{c}0.00^{* *} \\
(0.000)\end{array}$ & $\begin{array}{l}0.00^{* * *} \\
(0.000)\end{array}$ & $\begin{array}{c}0.00^{* *} \\
(0.001)\end{array}$ & $\begin{array}{l}0.00^{* * * *} \\
(0.001)\end{array}$ & y88 & $\begin{array}{l}-0.61^{* *} \\
(0.279)\end{array}$ & $\begin{array}{l}-0.56^{* *} \\
(0.270)\end{array}$ & $\begin{array}{l}-0.65^{* *} \\
(0.272)\end{array}$ & $\begin{array}{l}-0.63^{* *} \\
(0.265)\end{array}$ \\
\hline popgrowth & $\begin{array}{c}0.01 \\
(0.050)\end{array}$ & $\begin{array}{c}0.07 \\
(0.044)\end{array}$ & $\begin{array}{l}0.12 * * \\
(0.050)\end{array}$ & $\begin{array}{c}0.16 * * * \\
(0.045)\end{array}$ & y89 & $\begin{array}{c}-0.56^{*} \\
(0.298)\end{array}$ & $\begin{array}{l}-0.50^{*} \\
(0.291)\end{array}$ & $\begin{array}{l}-0.81^{* * *} \\
(0.311)\end{array}$ & $\begin{array}{l}-0.76^{* *} \\
(0.303)\end{array}$ \\
\hline oil price & $\begin{array}{l}0.01^{\text {*** }} \\
(0.002)\end{array}$ & $\begin{array}{c}0.00^{* *} \\
(0.002)\end{array}$ & $\begin{array}{l}0.01^{* * * *} \\
(0.003)\end{array}$ & $\begin{array}{l}0.01^{* *} \\
(0.002)\end{array}$ & y90 & $\begin{array}{c}-0.26 \\
(0.262)\end{array}$ & $\begin{array}{c}-0.17 \\
(0.233)\end{array}$ & $\begin{array}{c}-0.25 \\
(0.249)\end{array}$ & $\begin{array}{c}-0.19 \\
(0.231)\end{array}$ \\
\hline finance & $\begin{array}{l}0.17^{* * *} \\
(0.044)\end{array}$ & $\begin{array}{l}0.19^{* * *} \\
(0.042)\end{array}$ & $\begin{array}{l}0.14^{* * * *} \\
(0.052)\end{array}$ & $\begin{array}{l}0.16^{* * * *} \\
(0.051)\end{array}$ & y91 & $\begin{array}{c}-0.15 \\
(0.268)\end{array}$ & $\begin{array}{c}-0.08 \\
(0.239)\end{array}$ & $\begin{array}{c}-0.18 \\
(0.260)\end{array}$ & $\begin{array}{c}-0.13 \\
(0.245)\end{array}$ \\
\hline policy & & $\begin{array}{l}0.56^{* * *} \\
(0.070)\end{array}$ & & $\begin{array}{l}0.31^{\text {**** }} \\
(0.070)\end{array}$ & y92 & $\begin{array}{c}-0.25 \\
(0.294)\end{array}$ & $\begin{array}{c}-0.22 \\
(0.268)\end{array}$ & $\begin{array}{c}-0.12 \\
(0.271)\end{array}$ & $\begin{array}{c}-0.09 \\
(0.253)\end{array}$ \\
\hline patents & & & $\begin{array}{l}0.08^{* * *} \\
(0.013)\end{array}$ & $\begin{array}{l}0.06^{* * * *} \\
(0.013)\end{array}$ & y93 & $\begin{array}{c}-0.11 \\
(0.276)\end{array}$ & $\begin{array}{c}-0.08 \\
(0.260)\end{array}$ & $\begin{array}{c}-0.02 \\
(0.259)\end{array}$ & $\begin{array}{c}0.01 \\
(0.246)\end{array}$ \\
\hline enrollment & $\begin{array}{c}0.00 \\
(0.003)\end{array}$ & $\begin{array}{c}0.00 \\
(0.003)\end{array}$ & $\begin{array}{l}0.01^{* * * *} \\
(0.003)\end{array}$ & $\begin{array}{l}0.01^{* * * *} \\
(0.003)\end{array}$ & y94 & $\begin{array}{c}-0.04 \\
(0.270)\end{array}$ & $\begin{array}{c}-0.01 \\
(0.256)\end{array}$ & $\begin{array}{c}0.07 \\
(0.260)\end{array}$ & $\begin{array}{c}0.09 \\
(0.249)\end{array}$ \\
\hline hydro & $\begin{array}{l}-0.42^{* * *} \\
(0.083)\end{array}$ & $\begin{array}{l}-0.31^{* * *} \\
(0.079)\end{array}$ & $\begin{array}{l}-0.84^{* * *} \\
(0.072)\end{array}$ & $\begin{array}{l}-0.79^{* * *} \\
(0.070)\end{array}$ & y95 & $\begin{array}{c}0.05 \\
(0.269)\end{array}$ & $\begin{array}{c}0.08 \\
(0.253)\end{array}$ & $\begin{array}{c}0.23 \\
(0.240)\end{array}$ & $\begin{array}{c}0.25 \\
(0.229)\end{array}$ \\
\hline oil prod'n & $\begin{array}{c}-0.01^{\text {**** }} \\
(0.005)\end{array}$ & $\begin{array}{c}-0.01^{*} \\
(0.005)\end{array}$ & $\begin{array}{l}-0.03^{* * *} \\
(0.010)\end{array}$ & $\begin{array}{l}-0.03^{* *} \\
(0.010)\end{array}$ & y96 & $\begin{array}{c}0.02 \\
(0.254)\end{array}$ & $\begin{array}{c}0.03 \\
(0.236)\end{array}$ & $\begin{array}{c}0.13 \\
(0.233)\end{array}$ & $\begin{array}{c}0.13 \\
(0.219)\end{array}$ \\
\hline coal prod'n & $\begin{array}{l}0.09^{* * *} \\
(0.021)\end{array}$ & $\begin{array}{l}0.11^{* * *} \\
(0.023)\end{array}$ & $\begin{array}{l}0.10^{* * * *} \\
(0.037)\end{array}$ & $\begin{array}{l}0.12^{* * *} \\
(0.038)\end{array}$ & y97 & $\begin{array}{c}0.13 \\
(0.246)\end{array}$ & $\begin{array}{c}0.14 \\
(0.227)\end{array}$ & $\begin{array}{c}0.17 \\
(0.235)\end{array}$ & $\begin{array}{c}0.18 \\
(0.219)\end{array}$ \\
\hline FDI & & & $\begin{array}{l}-0.05^{* *} \\
(0.022)\end{array}$ & $\begin{array}{l}-0.05^{* *} \\
(0.021)\end{array}$ & y99 & $\begin{array}{c}0.20 \\
(0.226)\end{array}$ & $\begin{array}{c}0.20 \\
(0.215)\end{array}$ & $\begin{array}{c}0.20 \\
(0.224)\end{array}$ & $\begin{array}{c}0.20 \\
(0.214)\end{array}$ \\
\hline ODA & & & $\begin{array}{l}-0.02^{* *} \\
(0.012)\end{array}$ & $\begin{array}{l}-0.02^{* *} \\
(0.011)\end{array}$ & y00 & $\begin{array}{c}0.17 \\
(0.200)\end{array}$ & $\begin{array}{c}0.19 \\
(0.192)\end{array}$ & $\begin{array}{c}0.19 \\
(0.190)\end{array}$ & $\begin{array}{c}0.22 \\
(0.182)\end{array}$ \\
\hline coast & $\begin{array}{l}0.91^{\text {*** }} \\
(0.136)\end{array}$ & $\begin{array}{l}0.88^{* * *} \\
(0.136)\end{array}$ & $\begin{array}{l}0.94^{* * * *} \\
(0.150)\end{array}$ & $\begin{array}{l}0.92^{\text {**** }} \\
(0.149)\end{array}$ & y01 & $\begin{array}{c}0.32 \\
(0.197)\end{array}$ & $\begin{array}{c}0.28 \\
(0.185)\end{array}$ & $\begin{array}{c}0.37^{*} \\
(0.194)\end{array}$ & $\begin{array}{c}0.36^{*} \\
(0.183)\end{array}$ \\
\hline EAP & $\begin{array}{l}1.05^{* * *} \\
(0.141)\end{array}$ & $\begin{array}{l}0.82^{* * *} \\
(0.142)\end{array}$ & $\begin{array}{l}0.67^{* * * *} \\
(0.142)\end{array}$ & $\begin{array}{l}0.61^{\text {**** }} \\
(0.145)\end{array}$ & y02 & $\begin{array}{c}0.34^{*} \\
(0.188)\end{array}$ & $\begin{array}{c}0.29^{*} \\
(0.178)\end{array}$ & $\begin{array}{c}0.37^{*} \\
(0.189)\end{array}$ & $\begin{array}{c}0.34^{*} \\
(0.179)\end{array}$ \\
\hline LAC & $\begin{array}{l}1.33^{* * *} \\
(0.125)\end{array}$ & $\begin{array}{l}1.23^{* * *} \\
(0.119)\end{array}$ & $\begin{array}{l}1.34^{* * *} \\
(0.113)\end{array}$ & $\begin{array}{l}1.31^{* * * *} \\
(0.113)\end{array}$ & y03 & $\begin{array}{c}0.37^{*} \\
(0.190)\end{array}$ & $\begin{array}{c}0.32^{*} \\
(0.182)\end{array}$ & $\begin{array}{c}0.31 \\
(0.186)\end{array}$ & $\begin{array}{c}0.28 \\
(0.178)\end{array}$ \\
\hline ECA & $\begin{array}{l}0.48^{\text {*** }} \\
(0.157)\end{array}$ & $\begin{array}{l}0.42^{* * *} \\
(0.151)\end{array}$ & $\begin{array}{c}0.31^{*} \\
(0.172)\end{array}$ & $\begin{array}{c}0.27 \\
(0.175)\end{array}$ & y04 & $\begin{array}{c}0.29^{*} \\
(0.165)\end{array}$ & $\begin{array}{c}0.27^{*} \\
(0.156)\end{array}$ & $\begin{array}{c}0.24 \\
(0.164)\end{array}$ & $\begin{array}{c}0.22 \\
(0.155)\end{array}$ \\
\hline SA & $\begin{array}{l}0.98^{* * * *} \\
(0.177)\end{array}$ & $\begin{array}{l}0.56^{* * * *} \\
(0.190)\end{array}$ & $\begin{array}{c}0.21 \\
(0.190)\end{array}$ & $\begin{array}{c}0.08 \\
(0.201)\end{array}$ & $\mathrm{y} 05$ & $\begin{array}{c}0.14 \\
(0.142)\end{array}$ & $\begin{array}{c}0.15 \\
(0.131)\end{array}$ & $\begin{array}{c}0.14 \\
(0.143)\end{array}$ & $\begin{array}{c}0.14 \\
(0.131)\end{array}$ \\
\hline SSA & $\begin{array}{c}-0.23 \\
(0.173)\end{array}$ & $\begin{array}{l}-0.36^{* *} \\
(0.181)\end{array}$ & $\begin{array}{l}-0.49^{* *} \\
(0.219)\end{array}$ & $\begin{array}{l}-0.53^{* *} \\
(0.219)\end{array}$ & y06 & $\begin{array}{c}0.10 \\
(0.129)\end{array}$ & $\begin{array}{c}0.12 \\
(0.116)\end{array}$ & $\begin{array}{c}0.18 \\
(0.128)\end{array}$ & $\begin{array}{c}0.18 \\
(0.119)\end{array}$ \\
\hline y80 & $\begin{array}{l}-0.82^{* * *} \\
(0.214)\end{array}$ & $\begin{array}{l}-0.72^{* * *} \\
(0.206)\end{array}$ & $\begin{array}{l}-0.89^{* * *} \\
(0.225)\end{array}$ & $\begin{array}{c}-0.83^{* * *} \\
(0.216)\end{array}$ & y07 & $\begin{array}{c}0.03 \\
(0.125)\end{array}$ & $\begin{array}{c}0.06 \\
(0.115)\end{array}$ & $\begin{array}{c}0.18 \\
(0.128)\end{array}$ & $\begin{array}{c}0.19 \\
(0.120)\end{array}$ \\
\hline y81 & $\begin{array}{l}-0.87^{* * *} \\
(0.254)\end{array}$ & $\begin{array}{l}-0.76^{* * *} \\
(0.243)\end{array}$ & $\begin{array}{l}-0.83^{\text {*** }} \\
(0.265)\end{array}$ & $\begin{array}{l}-0.76^{* * *} \\
(0.256)\end{array}$ & y08 & $\begin{array}{c}-0.17 \\
(0.137)\end{array}$ & $\begin{array}{c}-0.05 \\
(0.123)\end{array}$ & $\begin{array}{c}-0.00 \\
(0.142)\end{array}$ & $\begin{array}{c}0.04 \\
(0.133)\end{array}$ \\
\hline y82 & $\begin{array}{l}-0.87^{* * *} \\
(0.251)\end{array}$ & $\begin{array}{l}-0.78^{* * *} \\
(0.241)\end{array}$ & $\begin{array}{l}-0.89^{* * *} \\
(0.268)\end{array}$ & $\begin{array}{l}-0.85^{* * *} \\
(0.260)\end{array}$ & y09 & $\begin{array}{c}0.22^{*} \\
(0.122)\end{array}$ & $\begin{array}{c}0.17 \\
(0.109)\end{array}$ & $\begin{array}{c}0.20^{*} \\
(0.121)\end{array}$ & $\begin{array}{c}0.16 \\
(0.113)\end{array}$ \\
\hline y83 & $\begin{array}{l}-0.66^{* * *} \\
(0.245)\end{array}$ & $\begin{array}{l}-0.59^{* *} \\
(0.237)\end{array}$ & $\begin{array}{l}-0.76^{* * * *} \\
(0.263)\end{array}$ & $\begin{array}{l}-0.73^{* * *} \\
(0.256)\end{array}$ & $\mathrm{y} 10$ & $\begin{array}{c}0.06 \\
(0.120)\end{array}$ & $\begin{array}{c}0.05 \\
(0.107)\end{array}$ & $\begin{array}{c}0.07 \\
(0.117)\end{array}$ & $\begin{array}{c}0.07 \\
(0.110)\end{array}$ \\
\hline y84 & $\begin{array}{l}-0.63^{* * *} \\
(0.232)\end{array}$ & $\begin{array}{l}-0.55^{\text {** }} \\
(0.224)\end{array}$ & $\begin{array}{l}-0.69^{\text {**** }} \\
(0.253)\end{array}$ & $\begin{array}{l}-0.66^{* * *} \\
(0.245)\end{array}$ & Constant & $\begin{array}{l}-4.35^{\text {**** }} \\
(0.466)\end{array}$ & $\begin{array}{l}-3.57^{* * *} \\
(0.458)\end{array}$ & $\begin{array}{c}4.37^{* *} \\
(1.851)\end{array}$ & $\begin{array}{c}3.95^{* *} \\
(1.818) \\
\end{array}$ \\
\hline y85 & $\begin{array}{l}-0.71^{* * * *} \\
(0.257)\end{array}$ & $\begin{array}{l}-0.65^{* * *} \\
(0.248)\end{array}$ & $\begin{array}{l}-0.72^{* * *} \\
(0.275)\end{array}$ & $\begin{array}{l}-0.69^{* * *} \\
(0.267)\end{array}$ & was adopt & $\begin{array}{l}\text { t standard } \\
.1 \text {. The ba } \\
\text { n the late } 1\end{array}$ & $\begin{array}{l}\text { ors in pare } \\
\text { year is } 199 \\
7 .\end{array}$ & $\begin{array}{l}\text { es, *** p } \\
\text { ause Kyo }\end{array}$ & P1, ** \\
\hline
\end{tabular}

